\title{
Analysis of the First Spectrum of Ruthenium (Ru I)
}

\author{
K. G. Kessler
}

(July 10, 1959)

\begin{abstract}
The analysis of the first spectrum of ruthenium has been extended with the aid of digital computers. A total of 105 even and 206 odd levels are listed with observed Landé $g$-factors for 54 even and 148 odd levels. A complete list of approximately 3,400 classified lines in the range 2013 to $11484 \mathrm{~A}$ is presented. The ionization limit calculated from a two member series is $59410 \mathrm{~cm}^{-1}$ or $7.364 \mathrm{v}$.
\end{abstract}

\section{Introduction}

Constant differences between wave numbers of line pairs in the are spectrum of ruthenium ( $\mathrm{Ru} \mathrm{I})$ were found by Kayser [1] ${ }^{1}$ in 1897 . Paulson [2], in 1915 , reported 17 recurring differences among the reciprocals of the ruthenium wavelengths in air. The first multiplets and energy levels were found by Meggers and Laporte [3, 4] who investigated the absorption spectrum to identify the ground-state transitions and assigned quantum numbers to 16 even and 24 odd levels. Simultaneously, Sommer $[5,6]$ published 44 even and 71 odd levels and identified spectral terms with the aid of Zeeman-effect data.

The next contribution to the analysis was by Harrison and McNally [7] who observed Zeeman patterns for 450 lines and published $g$-values for 38 even and 102 odd levels. With the aid of an interval sorter, MeNally later expanded this analysis [8] to include 61 even and 188 odd levels, accounting for 2,380 classified lines. This latter work has never been published, and was not made available to us until the present investigation was well under way.

\section{Experimental Procedure}

Line lists containing 4,492 ruthenium arc and 1,527 ruthenium spark lines compiled from spectrograms made at NBS [9] were used in this investigation. The observed wavelengths were converted to vacuum wave numbers by digital computers (the IBM 604, SEAC, and IBM 704 at various times) coded to compute the index of refraction of air by means of Edlen's formula [10]. An IBM 604 computer was then utilized to calculate all permitted transitions predicted by the known energy levels. These permitted transitions were compared with the observed lines in order to check the validity of these energy levels. All lines thus classified were removed from the line list to reduce this list to one containing only unclassified lines. Predictions which fell within $\pm 0.3 \mathrm{~cm}^{-1}$ were accepted as coincidences by the

Figures in brackets indicate the literature references on page 216. computer. Those which fell between \pm 0.2 and \pm 0.3 $\mathrm{cm}^{-1}$ were examined by the author and considered to be valid if adjacent lines in the multiplet were observed. The remaining list of unclassified Ru I lines was then searched for significant differences.

The search for new energy levels was carried out with the aid of electronic digital computers. The major portion of this work was done prior to 1957 with the NBS computer, the SEAC. The technique used is described elsewhere [11]. More recently, the faster IBM 704 computer has become available to us, and a revised and greatly expanded version of the technique used on the SEAC was developed for the newer computer by Coleman and Bozman [12]. The fundamental difference between the two computer techniques is that the earlier search was accomplished with a selected list of level differences, whereas the greater speed of the later machine permitted the use of all possible differences in the search procedure.

The list of unclassified lines remaining after the SEAC search has recently been investigated by means of the 704 routine. This search has yielded only five new even levels and one new odd level. It appears unlikely that more levels will be found with the present line list. More Zeeman data, particularly in the red and infrared regions, are needed for further progress on the classification of lines in this spectrum. New spectrograms devoid of underlying band spectra would also be helpful.

The energy levels, $g$-values, and percentage compositions for the low even levels calculated by Trees [13] have been very helpful in this analysis. For details of this, his paper in this issue (p. 255) should be consulted. For the rest of the assignments the author is indebted to Russell and Moore for the guidelines laid down in their analysis of the homologous spectrum, Fe I [14], and to C. E. Moore for her invaluable aid in grouping levels into terms and in assigning configurations.

The energy levels listed in table 1 have been adjusted to fit the new line list, but differ only slightly from those reported by McNally [8] in the case of those lines that occur in both lists. The estimated errors in the value of the levels range from \pm 0.01 
$\mathrm{cm}^{-1}$ for the low even levels to $\pm 0.05 \mathrm{~cm}^{-1}$ for those high terms that are determined by only a few transitions.

The array of observed terms is presented as table 2. To facilitate comparison, this array is given in the same form as the array of predicted terms published in A.E.L. [15].

The complete list of classified lines is given in table 3. The intensity of the observed line on an arbitrary scale of 1 to 10,000 , is listed in column 1 . The wavelength in column 2 and the corresponding wave number is listed in column 3 . The classification of the observed transition in terms of the energy levels listed in table 1 is given in column 4. A partial list of the 40 most intense remaining unclassified lines is given in table 4 .

\section{Discussion of Results}

\subsection{Even Terms}

The known parent terms in $\mathrm{Ru}$ II have proven a useful guide in assigning the $4 d^{7} 5 s$ configurations in $\mathrm{Ru}$ I. Terms can be assigned on the basis of the intensity of the combinations with a fair degree of certainty for the limits $a^{4} \mathbf{F}, a^{4} \mathrm{P}, a{ }^{2} \mathrm{G}, a{ }^{2} \mathrm{P}, a^{2} \mathrm{D}$, and $a^{2} \dot{H}$ in Ru II. A striking analogy exists with Fe I, although the term intervals are more irregular in Ru I. For these configurations, the levels are roughly 6000 to $12000 \mathrm{~cm}^{-1}$ lower than similar terms in Fe I.

All energy levels of $\mathrm{Ru} I$ which have been found are listed in table 1 . Term designations have been assigned when possible. The notation is identical with that used by C. E. Moore in the Atomic Energy Level Volumes [15], henceforth referred to as A.E.L. The configuration is given in the first column, term designation in the second, $J$-value in the third, energy level in the fourth, the term-intervals in the fifth and the observed $g$-value in the sixth column. The five miscellaneous even levels are designated with letters B through F. Energy levels of odd parity are given in italics. Miscellaneous odd levels are designated by numbers $1^{\circ}$ through $63^{\circ}$. The initials in the last column indicate the earliest reference for each level. The meaning of these initials is as follows: P, Paulson [2]; ML, Meggers and Laporte $[3,4]$; S, Sommer $[5,6]$; HM, Harrison and MeNally [7]; Mc, McNally [8]; and K, this paper.

This table is identical with that published in A.E.L. [15] except for the following changes: The levels $d{ }^{3} \mathrm{~F}_{2}, a{ }^{1} \mathrm{~F}_{3}, a{ }^{1} \mathrm{I}_{6}, e{ }^{5} \mathrm{G}_{4}, e{ }^{3} \mathrm{P}_{2}, \mathrm{~F}_{1}$ and $63^{\circ}{ }_{3}$ have been added, minor typographical errors in the energy level of $y{ }^{3} \mathrm{H}_{5}^{\circ}$ and in the $g$-value for $b^{3} \mathrm{H}_{6}$ have been corrected and the level formerly designated as $\mathrm{A}_{4}$ has been designated $f^{3} \mathrm{~F}_{4}$. The majority of the $g$-values are quoted from Harrison and McNally [7] and McNally [8]. The hitherto unpublished $g$-values from McNally's thesis together with a few additions contributed by the present author are included in the following paper. More Zeeman observations are needed in the red and infrared regions, where the present spectrograms record only very few lines.

The writer has benefited from consultations with Trees who has furnished theoretical predicted values for all of the low even terms. These calculations are based on parameters derived from known levels and are an excellent guide to the analysis. The general agreement of observation with his predictions is good. His paper [13] should be consulted for further details.

The IS coupling designations represent in general the major composition of each level, but some of the levels are mixed to such an extent that the LS notation should be used with caution. In particular, the designations of the levels $a^{3} \mathrm{D}_{2}$ at $15054.07 \mathrm{~cm}^{-1}$ and $a{ }^{1} \mathrm{D}_{2}$ at $17045.97 \mathrm{~cm}^{-1}$ should, according to Trees' calculations be interchanged. The relative intensities of the observed combinations and the term intervals, however, favor the designations assigned in table 1.

The level at $25200.97 \mathrm{~cm}^{-1}$ has been designated as $4 d^{7}\left(a{ }^{2} \mathrm{~F}\right) 5 s a^{1} \mathrm{~F}_{3}$ despite the absence of any observed combinations with singlet levels. This assignment was made on the strength of 'Trees' calculations which predict a level at $25210 \mathrm{~cm}^{-1}$ composed of 58 percent $4 d^{7}\left(a^{2} \mathrm{~F}\right) \quad 5 s^{1} \mathrm{~F}_{3}$ and 16 percent $4 d^{6} 5 s^{2}{ }^{3} \mathrm{G}_{3}$. The odd terms of similar parentage are unfortunately not well known.

The $a{ }^{5} \mathrm{D}$ term having the configuration $4 d^{6} 5 s^{2}$ is about $8000 \mathrm{~cm}^{-1}$ higher than the corresponding terms in Fe I. The level $a^{5} \mathrm{D}_{4}$, corresponding to the ground state of Fe I, occurs at $7483 \mathrm{~cm}^{-1}$ in $\mathrm{Ru} \mathrm{I}$. The remaining terms of this configuration are only slightly higher $\left(0\right.$ to $\left.3,000 \mathrm{~cm}^{-1}\right)$ than the corresponding terms in $\mathrm{Fe}$ I. Of the 16 terms predicted for this configuration, 8 have been identified but $d^{3} \mathrm{P}$ and $b^{3} \mathrm{D}$ are each missing one level and $f^{3} \mathrm{~F}$ is missing two levels. Of the 8 singlets predicted, only $a^{1} I$ has been found. According to 'Trees' predictions, the higher ${ }^{1} \mathrm{~S}$ and ${ }^{1} \mathrm{D}$ terms will lie above the ionization limit. The other missing singlet terms are ${ }^{1} \mathrm{~S}$ predicted near $33411 \mathrm{~cm}^{-1}$, ${ }^{1} \mathrm{D}$ near $33905 \mathrm{~cm}^{-1},{ }^{1} \mathrm{~F}$ near $39308 \mathrm{~cm}^{-1}$ and two ${ }^{1} \mathrm{G}$ terms, one near 31719 , the other near $48417 \mathrm{~cm}^{-1}$. A high ${ }^{3} \mathrm{P}$ term predicted near $43571 \mathrm{~cm}^{-1}$ is also missing.

The $b^{3} \mathrm{D}_{3}$ level deviates sufficiently, by $582 \mathrm{~cm}^{-1}$, from Trees' value to cast some doubt upon its validity. A level at $29617.15 \mathrm{~cm}^{-1}$ which might possibly be designated as $4 d^{6} 5 s^{2} b^{3} \mathrm{D}_{1}$ has not been included in table 1 . The agreement with the predicted position, $29621 \mathrm{~cm}^{-1}$, is good, but only three weak combinations with $w^{3} \mathrm{D}_{2}^{\circ}, \quad z^{1} \mathrm{D}_{2}^{\circ}$, and $y{ }^{1} \mathrm{P}_{1}^{\circ}$ have been observed and further confirmation is needed.

The $c{ }^{3} \mathrm{~F}_{2}$ and $c{ }^{3} \mathrm{~F}_{3}$ levels deviate by 149 and 173 $\mathrm{cm}^{-1}$ from their predicted positions and both are strongly mixed with $4 d^{7}\left(a^{2} \mathrm{~F}\right) 5 s, d^{3} \mathrm{~F}_{2}$ and $d^{3} \mathrm{~F}_{3}$. The average deviation of the remaining 18 levels of the $4 d^{6} 5 s^{2}$ configuration from their predicted positions is $45 \mathrm{~cm}^{-1}$.

All terms of the $4 d^{8}$ configuration have been found with the exception of ${ }^{1} \mathrm{~S}$. This term should occur at about $38892 \mathrm{~cm}^{-1}$. The $c^{3} \mathrm{P}_{\mathrm{O}}$ level does not agree 
well with Trees' calculations. The evidence for this level consists of only three transitions, but since extensive searching revealed no other level with $J=0$ in this region, it was kept in the list with a question mark. The remaining seven levels deviate by less than $37 \mathrm{~cm}^{-1}$ from Trees' predictions. The only term from this configuration known in Fe I lies $23500 \mathrm{~cm}^{-1}$ above the corresponding term, $b^{3} \mathrm{~F}$, in $\mathrm{Ru} \mathrm{I}$.

Only the terms $e^{7} \mathrm{D}$ and $f^{5} \mathrm{D}$ from the $4 d^{6} 5^{s}\left(a^{6} \mathrm{D}\right) 6 s$ configuration are known, and the latter is incomplete. They are about $6000 \mathrm{~cm}^{-1}$ higher than the corresponding terms in Fe I.

The remaining even configurations observed involve a $5 d$ electron. The terms with this configuration are found above $47000 \mathrm{~cm}^{-1}$ and are difficult to find with certainty because of the limited number of expected transitions. Six of the ten predicted terms with the configuration $4 d^{7}\left(a^{4} \mathrm{~F}\right) 5 d$ are represented in the tables, but only one, $e^{3} \mathrm{G}$, is complete. They lie about $6000 \mathrm{~cm}^{-1}$ below those in Fe I. Data on the remaining $5 d$ configurations are extremely fragmentary. Two terms, $e^{5} \mathrm{~S}$ and part of $f^{7} \mathrm{D}$ are known and tentatively assigned to $4 d^{6} 5 s\left(a^{6} \mathrm{D}\right) 5 d$. They lie almost coincident with the similar terms in $\mathrm{Fe} \mathrm{I}$.

A possible level at $55405.64 \mathrm{~cm}^{-1}$ may be the missing $4 d^{7}\left(a{ }^{4} \mathrm{P}\right) 5_{p} \quad f^{3} \mathrm{D}_{3}^{\circ}$ level. A transition from. a level at this position to $y^{3} \mathrm{D}_{3}^{\circ}$ would coincide with the position of the strongest remaining unclassified line at $5361 \mathrm{~A}$. Very weak lines at the wavelengths corresponding to combinations with three other levels, $y{ }^{5} \mathrm{P}_{3}^{\circ}, y{ }^{5} \mathrm{P}_{2}^{\circ}$, and $6_{3}^{\circ}$, have been found. These combinations may, however, be coincidental and the level has not been listed in table 1 .

The strongest remaining unclassified lines lie between 4700 and $7500 \mathrm{~A}$. Many of these probably represent transitions between unidentified terms with a $4 d^{7}\left(a{ }^{4} \mathrm{~F}\right) 5 d, 4 d^{7}\left(a{ }^{4} \mathrm{P}\right) 5 d$, or a $4 d^{6} 5 s\left(a{ }^{6} \mathrm{D}\right) 5 d$ configuration and odd terms from the same limits. These multiplets are characterized by strong transitions on the main diagonal with very weak offdiagonal transitions. The corresponding energy levels are, therefore, difficult to find.

\subsection{Odd Terms}

All terms of the $4 d^{7} 5 p$ configuration having as limits in Ru II $a{ }^{4} \mathrm{~F}, a{ }^{4} \mathrm{P}, a{ }^{2} \mathrm{G}, a^{2} \mathrm{D}$, and $a^{2} \mathrm{H}$ have been found. The intervals are approximately three times as large as those in Fe I. Those from $a^{4} \mathrm{~F}$ lie about $5500 \mathrm{~cm}^{-1}$ below the corresponding terms in Fe I. The two levels $z^{5} \mathrm{G}_{3}^{0}$ and $z^{5} \mathrm{G}_{2}^{0}$ appear to be perturbed downward.

In the next group, i.e., $4 d^{7}\left(a^{4} \mathrm{P}\right) 5 p$, the lowest term., $z^{5} \mathrm{~S}^{\circ}$, is $13326 \mathrm{~cm}^{-1}$ below the analogous term in $\mathrm{Fe} \mathrm{I}$; the rest are about $9000 \mathrm{~cm}^{-1}$ lower. Also, as in Fe $\mathrm{I}$, the levels of $x^{5} \mathrm{D}^{\circ}$ appear to be strongly perturbed.

For $4 d^{7}\left(a^{2} \mathrm{G}\right) 5 p$, the terms are about $8000 \mathrm{~cm}^{-1}$ below those in Fe I, and the intervals of the terms $y^{3} \mathrm{G}^{\circ}$ and $z^{3} \mathrm{H}^{\circ}$ show evidence of severe perturbation.

In the $4 d^{7}\left(a^{2} \mathrm{P}\right) 5 p$ configuration, all levels lie about $9000 \mathrm{~cm}^{-1}$ below those that have been found in Fe I. The intervals of the $x^{3} \mathrm{D}^{\circ}$ term show evidence of strong perturbation.

The terms of the configuration $4 d^{7}\left(a^{2} \mathrm{H}\right) 5 p$ lie about $8000 \mathrm{~cm}^{-1}$ below those in Fe I. The intervals of $z^{3} \mathrm{I}^{\circ}$ indicate strong perturbation.

The levels $x^{3} \mathrm{P}_{1}^{\circ}$ and $x^{3} \mathrm{P}_{0}^{\circ}$ of the $4 d^{7}\left(a^{2} \mathrm{D}\right) 5 p$ configuration are missing. The remaining terms range 6000 to $15000 \mathrm{~cm}^{-1}$ below those in $\mathrm{Fe} \mathrm{I}$, and the intervals of the $x^{3} \mathrm{~F}^{\circ}$ term indicate a strong perturbation.

Only two terms, $x{ }^{1} \mathrm{P}^{\circ}$ and $x^{1} \mathrm{G}^{\circ}$ of the $4 d^{7}\left(\mathrm{a}^{2} \mathrm{~F}\right) 5 p$ configuration are suggested and these assignments are tentative. No levels of this configuration have been classified in Fe I.

For the configuration $4 d^{6} 5 s 5 p$ the two triads of septet and quintet terms having as limit $a^{6} \mathrm{D}$ in Ru II are well known. These terms are regular and lie from 6500 to $8500 \mathrm{~cm}^{-1}$ above the related terms in $\mathrm{Fe} \mathrm{I}$.

For the $4 d^{6} 5 s\left(a^{4} \mathrm{D}\right) 5 p$ configuration, only two terms, $\mathrm{w}^{5} \mathrm{D}^{\circ}$ and $x^{5} \mathrm{~F}^{\circ}$ have been designated and the level $x^{5} \mathrm{~F}_{1}$ is missing. These terms lie about $5000 \mathrm{~cm}^{-1}$ above those in Fe I. Similarly, only two terms, $y{ }^{5} \mathrm{~S}^{\circ}$ and $x^{5} \mathrm{p}^{\circ}$ are ascribed to $4 d^{6} 5 s\left(b^{4} \mathrm{P}\right) 5 p$. They are about $4000 \mathrm{~cm}^{-1}$ above those in Fe $\mathrm{I}$. The unassigned term $t^{3} \mathrm{D}^{\circ}$ may belong in this group.

One term, $x^{1} \mathrm{H}^{\circ}$ is ascribed to $4 d^{6} 5 s\left(b^{2} \mathrm{H}\right) 5 p$ because of its strong combination with $a^{1} \mathrm{H}$.

Five terms are entered in table 1 without configuration assignments: $v^{3} \mathrm{D}^{\circ}, u^{3} \mathrm{D}^{\circ}, t^{3} \mathrm{D}^{\circ}, w^{3} \mathrm{G}^{\circ}$, and $v^{3} \mathrm{G}^{\circ}$. All of these are fragmentary but observed $g$-values are known for seven of the levels involved.

The remaining odd levels have been classed as miscellaneous. These levels lie above $40000 \mathrm{~cm}^{-1}$ and most of them are probably fragments of the various remaining $4 d^{6} 5 s 5 p$ or $4 d^{7}\left(a^{2} \mathrm{~F}\right) 5 p$ configurations. Some possible assignments, made on the basis of the intensities of the observed combinations, are given in parentheses. Very few Zeeman effect data are available in this range, and the overlapping of terms is so great that configuration and term assignments have little significance.

\subsection{Ionization Limits}

The ionization limit for $\mathrm{Ru}$ I was determined by fitting the observed term values for the two series members $4 d^{7}\left(a^{4} \mathrm{~F}\right) 5 s \quad a^{5} \mathrm{~F}_{5}\left(T_{1}^{\prime}=0.0 \quad \mathrm{~cm}^{-1}\right) \quad$ and $4 d^{7}\left(a^{4} \mathrm{~F}\right) 6 s \quad e^{5} \mathrm{~F}_{5}\left(T_{2}^{\prime}=41256.40 \mathrm{~cm}^{-1}\right)$ to the formulas below. The value of the constant $\alpha=-2.43$ $\times 10^{-6} \mathrm{~cm}$ used is an interpolated value obtained by Catalan and Rico [16]. This interpolation was obtained by drawing a smooth curve through the points given by the available data on a plot of $\alpha$ versus atomic number for the first spectra of the elements $\mathrm{Rb}$ through $\mathrm{Ag}$.

$$
\begin{aligned}
& T_{n}=R /\left(n+\mu+\alpha T_{n}\right)^{2} \\
& T_{n}=T_{\infty}-T_{n}^{\prime}
\end{aligned}
$$


where: $T_{n}$ is the energy level of the $n$th series member measured relative to the ionization limit, $T_{\infty}$,

$T_{n}^{\prime}$ is the energy level of the $n$th series member measured relative to the ground state,

$\mu$ is the quantum defect and is assumed to be constant for the series,

$\alpha$ is a constant,

$R$ is the Rydberg constant, and

$n$ is 1 for the first term, 2 for the second term.

This set of equations is satisfied by a unique value of the ionization limit, $T_{\infty}$. The value of the ionization limit obtained in this manner is $59410 \mathrm{~cm}^{-1}$ or $7.364 \mathrm{ev}$. This value of the ionization limit agrees well with the value $59417 \mathrm{~cm}^{-1}$ obtained by Catalan and Rico by interpolation between data from spectra of neighboring elements in the periodic table.

The author acknowledges C. E. Moore's invaluable help in assigning configurations and in arranging the list of energy levels into appropriate terms and R. E. Trees' excellent calculations of levels and compositions for the low even configurations. The author also thanks W. F. Meggers for his aid in assembling the line list and W. R. Bozman for his help in operating the 704 computer.

\section{References}

[1] H. Kayser, Astrophys. J. 7, 93-113, 173-197 (1898).

[2] E. Paulson, Physik. Z. 16, 81 (1915).

[3] W. F. Meggers and O. Laporte, Science 61, 635 (1925).

[4] W. F. Meggers and O. Laporte, J. Wash. Acad. Sci. 16, 143 (1926).

[5] L. A. Sommer, Naturwiss. 13, 840 (1925).

[6] L. A. Sommer, Z. Physik 37, 1 (1926).

[7] G. R. Harrison and J. R. MeNally, Jr., Phys. Rev. 58, 703 (1940).

[8] J. R. MeNally, Jr., Thesis, Mass, Inst. Technol. (1941) unpublished.

[9] K. G. Kessler and W. F. Meggers, J. Research NBS 55, 97 (1955) RP 2609.

[10] B. Edlén, Minutes, Trans. Joint Com. for Spectroscopy, J. Opt. Soc. Am. 43, 410 (1953)

[11] K. G. Kessler, S. B. Prusch, and I. A. Stegun, J. Opt. Soc. Am. 46, 1043 (1956).

[12] W. D. Coleman and W. R. Bozman, J. Opt. Soc. Am. 49, 511 (1959).

[13] R. E. Trees, J. Opt. Soc. Am. 49, 838 (1959) ; J. Research NBS 63A, 255 (1959)

[14] H. N. Russell and C. E. Moore, Trans. Am. Phil. Soc. 34, pt 11, 111 (1944).

[15] C. E. Moore, Atomic Energy Levels III, NBS Circ. 467, (1958).

[16] M. A. Catalán and F. R. Rico, Anales Soc. Esp. de fís. y quím A48, 328 (1952). 
Table 1

$\mathrm{Ru}$ I

\begin{tabular}{|c|c|c|c|c|c|c|c|c|c|c|c|c|c|}
\hline Config. & Desig. & $J$ & Level & Interval & Obs. $g$ & $\begin{array}{l}\text { Earliest } \\
\text { reference }\end{array}$ & Config. & Desig. & $J$ & Level & Interval & Obs. $g$ & $\begin{array}{l}\text { Earliest } \\
\text { reference }\end{array}$ \\
\hline $4 d^{7}\left(a^{4} \mathrm{~F}\right) 5 s$ & $a \quad{ }^{5} \mathrm{~F}$ & $\begin{array}{l}5 \\
4 \\
3 \\
2 \\
1\end{array}$ & $\begin{array}{r}0.00 \\
\text { 1190. } 64 \\
\text { 2091. } 54 \\
2713.24 \\
3105.49\end{array}$ & $\begin{array}{r}-1190.64 \\
-900.90 \\
-621.70 \\
-392.25\end{array}$ & $\begin{array}{l}\text { 1. } 397 \\
\text { 1. } 349 \\
\text { 1. } 249 \\
\text { 1. } 000\end{array}$ & $\begin{array}{l}\mathrm{P} \\
\mathrm{P} \\
\mathrm{P} \\
\mathrm{P}\end{array}$ & $4 d^{8}$ & $c{ }^{3} \mathrm{P}$ & $\begin{array}{l}2 \\
1 \\
0\end{array}$ & $\begin{array}{l}20933.75 \\
22292.64 \\
24173.68 ?\end{array}$ & $\begin{array}{l}-1358.89 \\
-1881.04 ?\end{array}$ & $\begin{array}{l}\text { 1. } 343 \\
\text { 1. } 35\end{array}$ & $\begin{array}{l}\mathrm{H} M \\
\mathrm{~K} \\
\mathrm{~K}\end{array}$ \\
\hline \multirow[t]{2}{*}{$4 d^{7}\left(a^{4} \mathrm{~F}\right) 5 s$} & \multirow[t]{2}{*}{$a{ }^{3} \mathrm{~F}$} & $\begin{array}{l}1 \\
4 \\
3\end{array}$ & $\begin{array}{l}3105.49 \\
6545.03 \\
8084.12\end{array}$ & -1539.09 & $\begin{array}{l}0.000 \\
\text { 1. } 284 \\
1.196\end{array}$ & $\begin{array}{l}\text { ML, S } \\
\mathrm{P} \\
\mathrm{P}\end{array}$ & $4 d^{6} 5 s^{2}$ & $c{ }^{3} \mathrm{~F}$ & $\begin{array}{l}4 \\
3 \\
2\end{array}$ & $\begin{array}{l}21643.09 \\
22419.46 \\
22343.14\end{array}$ & $\begin{array}{r}-776.37 \\
76.32\end{array}$ & $\begin{array}{l}\text { 1. } 08 \\
\text { 1. } 070 \\
0.697\end{array}$ & $\begin{array}{l}\mathrm{K} \\
\mathrm{K} \\
\mathrm{K}\end{array}$ \\
\hline & & $\begin{array}{l}2 \\
4\end{array}$ & $\begin{array}{l}9183.66 \\
7483.07\end{array}$ & -1099.54 & $\begin{array}{l}\text { 1. } 089 \\
\text { 1. } 447\end{array}$ & $\begin{array}{l}\mathrm{P} \\
\mathrm{P}\end{array}$ & $4 d^{6} 5 s^{2}$ & $b \quad{ }^{3} \mathrm{H}$ & $\begin{array}{l}6 \\
5 \\
4\end{array}$ & $\begin{array}{l}22162.06 \\
22518.88 \\
23004.77\end{array}$ & \multirow[t]{4}{*}{$\begin{array}{l}-356.82 \\
-485.89\end{array}$} & $\begin{array}{l}\text { 1. } 063 \\
0.99 \\
0.91 \text { ? }\end{array}$ & $\begin{array}{l}\mathrm{K} \\
\mathrm{K} \\
\mathrm{K}\end{array}$ \\
\hline \multirow{3}{*}{$4 d^{6} 5 s^{2}$} & \multirow{3}{*}{$a \quad{ }^{5} \mathrm{D}$} & $\begin{array}{l}3 \\
2\end{array}$ & $\begin{array}{l}8575.42 \\
9057.64\end{array}$ & $\begin{array}{r}-1092.35 \\
-482.22\end{array}$ & 1. 420 & & $4 d^{8}$ & & & & & & \\
\hline & & $\begin{array}{l}2 \\
1\end{array}$ & $\begin{array}{l}9058.64 \\
9072.98\end{array}$ & -15. 34 & $\begin{array}{l}\text { 1. } 2.32 \\
1.795\end{array}$ & $\mathrm{ML}, \mathrm{S}$ & $4 d^{8}$ & $b \quad{ }^{1} \mathrm{G}$ & 4 & 23392. 60 & & 0.950 & K \\
\hline & & 0 & 9492.37 & -419.39 & $0 / 0$ & $\mathrm{HM}$ & $4 d^{8}$ & $b \quad{ }^{1} \mathrm{D}$ & 2 & 23453. 47 & & 1. 162 & K \\
\hline $4 d^{7}\left(a^{4} \mathrm{P}\right) 5 s$ & $a \quad{ }^{5} \mathrm{P}$ & $\begin{array}{l}3 \\
2 \\
1\end{array}$ & $\begin{array}{l}8770.93 \\
8043.69 \\
9620.29\end{array}$ & $\begin{array}{r}727.24 \\
-1576.60\end{array}$ & $\begin{array}{l}\text { 1. } 624 \\
\text { 1. } 536 \\
\text { 1. } 985\end{array}$ & $\begin{array}{l}\mathrm{P} \\
\mathrm{P} \\
\mathrm{ML}, \mathrm{S}\end{array}$ & $4 d^{6} 5 s^{2}$ & $d \quad{ }^{3} \mathrm{P}$ & $\begin{array}{l}2 \\
1 \\
0\end{array}$ & $\begin{array}{l}24927.48 \\
27560.59\end{array}$ & -2633.11 & & $\begin{array}{l}\mathrm{K} \\
\mathrm{K}\end{array}$ \\
\hline \multirow[t]{2}{*}{$4 d^{8}$} & \multirow[t]{2}{*}{$b \quad{ }^{3} \mathrm{~F}$} & $\begin{array}{l}4 \\
3\end{array}$ & $\begin{array}{r}9120.63 \\
10654.62\end{array}$ & -1533.99 & $\begin{array}{l}\text { 1. } 255 \\
\text { 1. } 086\end{array}$ & $\stackrel{\mathrm{P}}{\mathrm{P}}$ & \multirow{3}{*}{$\begin{array}{l}4 d^{7}\left(a^{2} \mathrm{~F}\right) 5 s \\
4 d^{6} 5 s\left(a^{6} \mathrm{D}\right) 5 p\end{array}$} & \multirow{3}{*}{$\begin{array}{l}a \quad{ }^{1} \mathrm{~F} \\
z \quad 7 \mathrm{D}^{\circ}\end{array}$} & 3 & 25200.97 & & & K \\
\hline & & 2 & 11447. 31 & -792. 69 & 0.764 & $\mathrm{P}$ & & & $\begin{array}{l}5 \\
4\end{array}$ & 25214.16 & -250.33 & 1. 592 & $\mathrm{HM}_{\mathrm{M}}$ \\
\hline $4 d^{7}\left(a^{2} \mathrm{P}\right) 5 s$ & $a{ }^{3} \mathrm{P}$ & $\begin{array}{l}2 \\
1 \\
0\end{array}$ & $\begin{array}{l}\text { 10623. } 53 \\
11786.05 \\
11752.62\end{array}$ & $\begin{array}{r}-1162.52 \\
33.43\end{array}$ & $\begin{array}{l}\text { 1. } 534 \\
\text { 1. } 684 \\
0 / 0\end{array}$ & $\begin{array}{l}\mathrm{P} \\
\mathrm{S} \\
\mathrm{S}\end{array}$ & & & $\begin{array}{l}4 \\
3 \\
2 \\
1\end{array}$ & $\begin{array}{l}20404.49 \\
26035.56 \\
26472.74 \\
26780.46\end{array}$ & $\begin{array}{l}-571.07 \\
-437.18 \\
-307.72\end{array}$ & $\begin{array}{l}\text { 1. } 020 \\
\text { 1. } 737 \\
\text { 1. } 992\end{array}$ & $\begin{array}{l}\text { SiL, } \\
\text { MC } \\
\text { MC }\end{array}$ \\
\hline $4 d^{7}\left(a^{2} \mathrm{G}\right) 5 s$ & $a{ }^{3} \mathrm{G}$ & $\begin{array}{l}5 \\
4 \\
3\end{array}$ & $\begin{array}{l}\text { 12207. } 05 \\
12816.69 \\
13699.07\end{array}$ & $\begin{array}{l}-609.64 \\
-882.38\end{array}$ & $\begin{array}{l}\text { 1. } 190 \\
\text { 1. } 033 \\
0.757\end{array}$ & $\begin{array}{l}\text { HM } \\
\text { S } \\
\text { HM }\end{array}$ & $4 d^{6} 5 s^{2}$ & $b \quad{ }^{3} \mathrm{G}$ & $\begin{array}{l}5 \\
4 \\
3\end{array}$ & $\begin{array}{l}25602.60 \\
25642.69 \\
26075.70\end{array}$ & $\begin{array}{r}-40.09 \\
-433.01\end{array}$ & & $\begin{array}{l}\mathrm{K} \\
\mathrm{K} \\
\mathrm{K}\end{array}$ \\
\hline \multirow[t]{2}{*}{$4 d^{7}\left(a^{4} \mathrm{P}\right) 5 s$} & $b \quad{ }^{3} \mathrm{P}$ & 2 & 13645. 75 & -335.92 & 1. 315 & & $4 d^{7}\left(a^{4} \mathrm{~F}\right) 5 p$ & $z{ }^{5} \mathrm{D}^{\circ}$ & 4 & 26312. 83 & $-1193,76$ & 1. 486 & $\mathrm{ML}, \mathrm{S}$ \\
\hline & & $\begin{array}{l}1 \\
0\end{array}$ & $\begin{array}{l}13981.67 \\
14827.50\end{array}$ & -845.83 & $\begin{array}{l}\text { 1. } 441 \\
0 / 0\end{array}$ & $\stackrel{\mathrm{S}}{\mathrm{H} M}$ & & & $\begin{array}{l}3 \\
2 \\
1\end{array}$ & $\begin{array}{l}27506.59 \\
28465.69\end{array}$ & $\begin{array}{r}-1195.10 \\
-959.10 \\
-652.80\end{array}$ & $\begin{array}{l}\text { 1. } 425 \\
\text { 1. } 324\end{array}$ & $\begin{array}{l}\text { ML, S } \\
\text { ML, S }\end{array}$ \\
\hline $4 d^{7}\left(a^{2} \mathrm{G}\right) 5 s$ & $a{ }^{1} \mathrm{G}$ & 4 & 14700.32 & & 0. 992 & H M & & & $\begin{array}{l}1 \\
0\end{array}$ & $\begin{array}{l}29118.49 \\
29569.90\end{array}$ & -451.41 & $\begin{array}{l}0.953 \\
0 / 0\end{array}$ & $\begin{array}{l}\text { ML, S } \\
\text { ML }\end{array}$ \\
\hline $4 d^{7}\left(a^{2} \mathrm{D}\right) 5 s$ & $a{ }^{3} \mathrm{D}$ & 3 & 16190. 61 & & 1. 333 & HM & $4 d^{7}\left(a^{4} \mathrm{~F}\right) 5 p$ & $z{ }^{5} \mathrm{~F}^{\circ}$ & 5 & 26816. 23 & & 1. 394 & ML, S \\
\hline & & $\begin{array}{l}2 \\
1\end{array}$ & $\begin{array}{l}15054.07 \\
16712.58\end{array}$ & -1658.51 & $\begin{array}{l}\text { 1. } 162 \\
0.676\end{array}$ & $\begin{array}{l}\mathrm{S} \\
\mathrm{HM}\end{array}$ & & & $\begin{array}{l}4 \\
3 \\
0\end{array}$ & $\begin{array}{l}28014.79 \\
28890.47\end{array}$ & $\begin{array}{r}-1190.00 \\
-875.68 \\
-536.85\end{array}$ & $\begin{array}{l}\text { 1. } 364 \\
\text { 1. } 293\end{array}$ & $\begin{array}{l}\text { ML, S } \\
\text { ML, S }\end{array}$ \\
\hline $4 d^{7}\left(a^{2} \mathrm{H}\right) 5 \mathrm{~s}$ & $a{ }^{3} \mathrm{H}$ & $\begin{array}{l}6 \\
5\end{array}$ & $\begin{array}{l}15550.16 \\
16240.13\end{array}$ & -689.97 & $\begin{array}{l}\text { 1. } 164 \\
\text { 1. } 041\end{array}$ & $\begin{array}{l}\mathrm{HM} \\
\mathrm{HM}\end{array}$ & & & $\begin{array}{l}2 \\
1\end{array}$ & $\begin{array}{l}29427.32 \\
29693.57\end{array}$ & $\begin{array}{l}-266.25 \\
-200\end{array}$ & $\begin{array}{l}\text { 1. } 164 \\
0.567\end{array}$ & $\begin{array}{l}\text { ML, S } \\
\text { ML, S }\end{array}$ \\
\hline & & 4 & 17096.87 & -856.74 & 0.834 & $\mathrm{HM}$ & $4 d^{7}\left(a^{2} \mathrm{~F}\right) 5 s$ & $d \quad{ }^{3} \mathrm{~F}$ & 4 & 27289. 24 & -227.33 & & $\mathrm{~K}$ \\
\hline $4 d^{7}\left(a^{2} \mathrm{D}\right) 5 s$ & $a \quad 1 \mathrm{D}$ & 2 & 17045.97 & & 1. 175 & HM & & & $\begin{array}{l}3 \\
2\end{array}$ & 26815. 67 & 700.90 & & $\begin{array}{l}\mathrm{K} \\
\mathrm{K}\end{array}$ \\
\hline $4 d^{7}\left(a^{2} \mathrm{H}\right) 5 s$ & $a{ }^{1} \mathrm{H}$ & 5 & 20055. 71 & & 1. 007 & $\mathrm{MC}$ & $4 d^{7}\left(a^{4} \mathrm{~F}\right) 5 p$ & $z^{3} \mathrm{G}^{\circ}$ & 5 & 28495.10 & -1395.81 & 1. 230 & ML, S \\
\hline $4 d^{7}\left(a^{2} \mathrm{P}\right) 5 s$ & $a{ }^{1} \mathrm{P}$ & 1 & 20242. 01 & & 0.927 & HM & & & $\begin{array}{l}4 \\
3\end{array}$ & $\begin{array}{l}29890.91 \\
31852.90\end{array}$ & -1961.99 & 0.868 & $\begin{array}{l}\text { ML, S } \\
\text { ML, S }\end{array}$ \\
\hline
\end{tabular}


TABLE 1-Continued

$\mathrm{R}_{\mathrm{I}}$

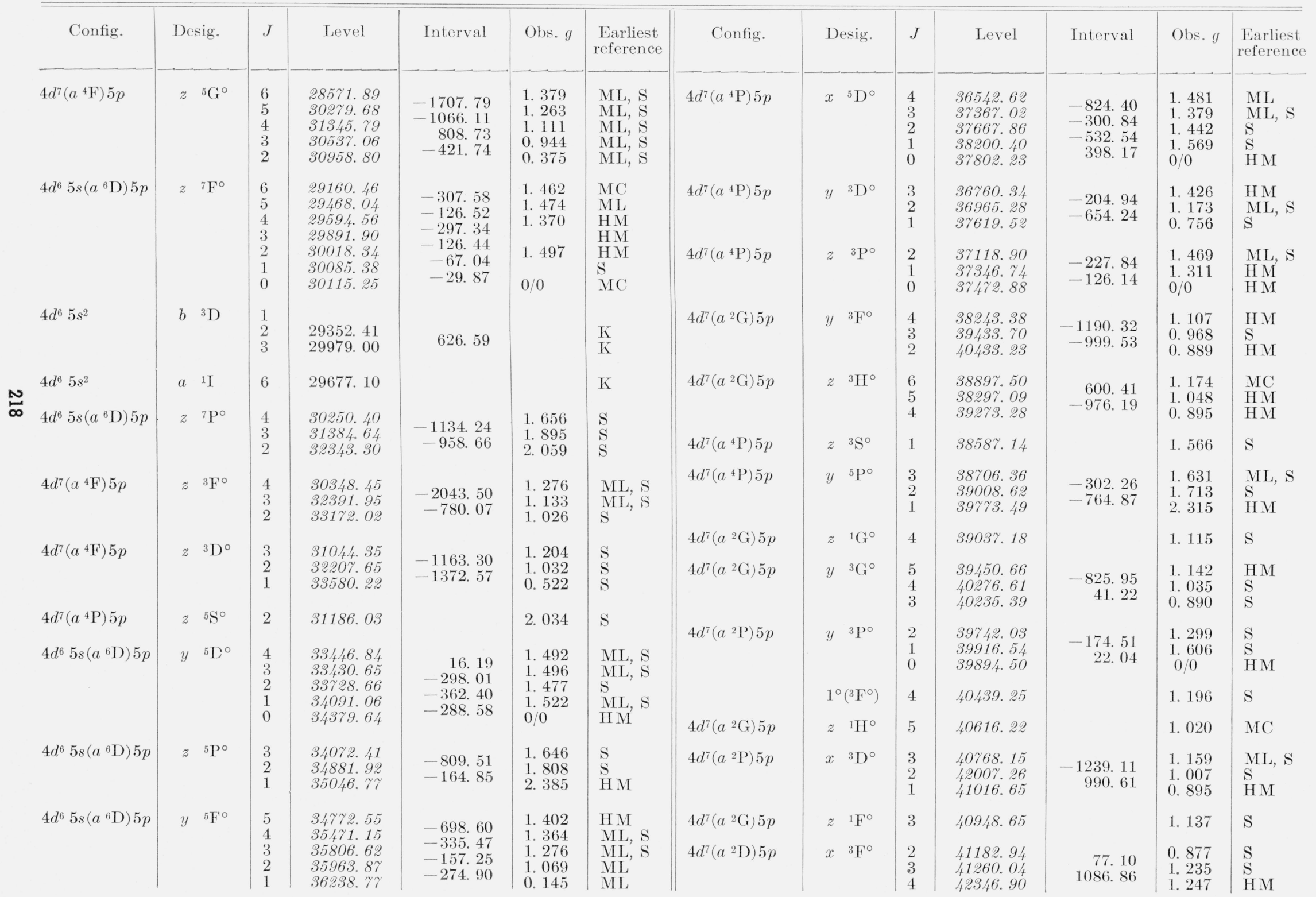




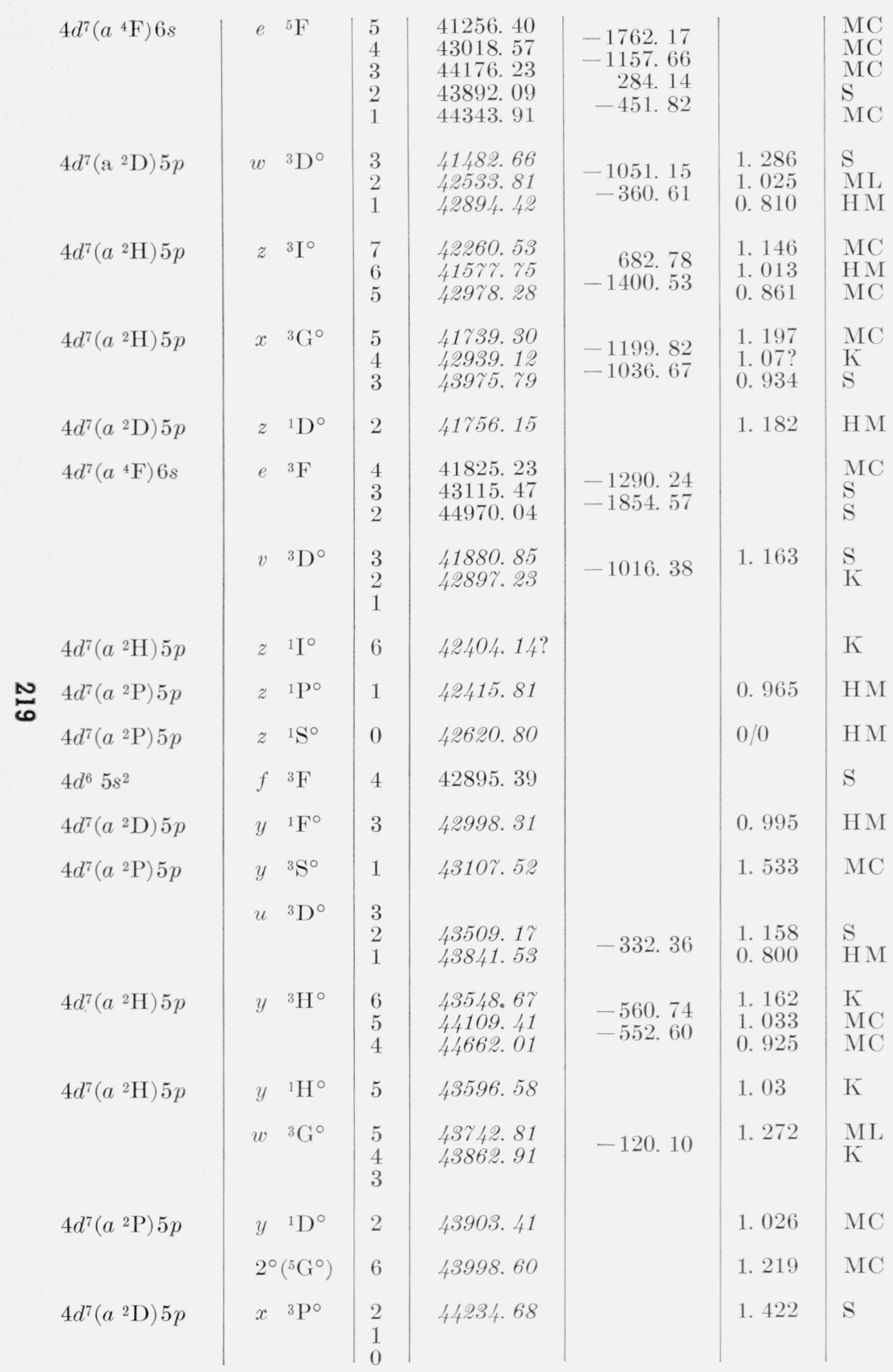

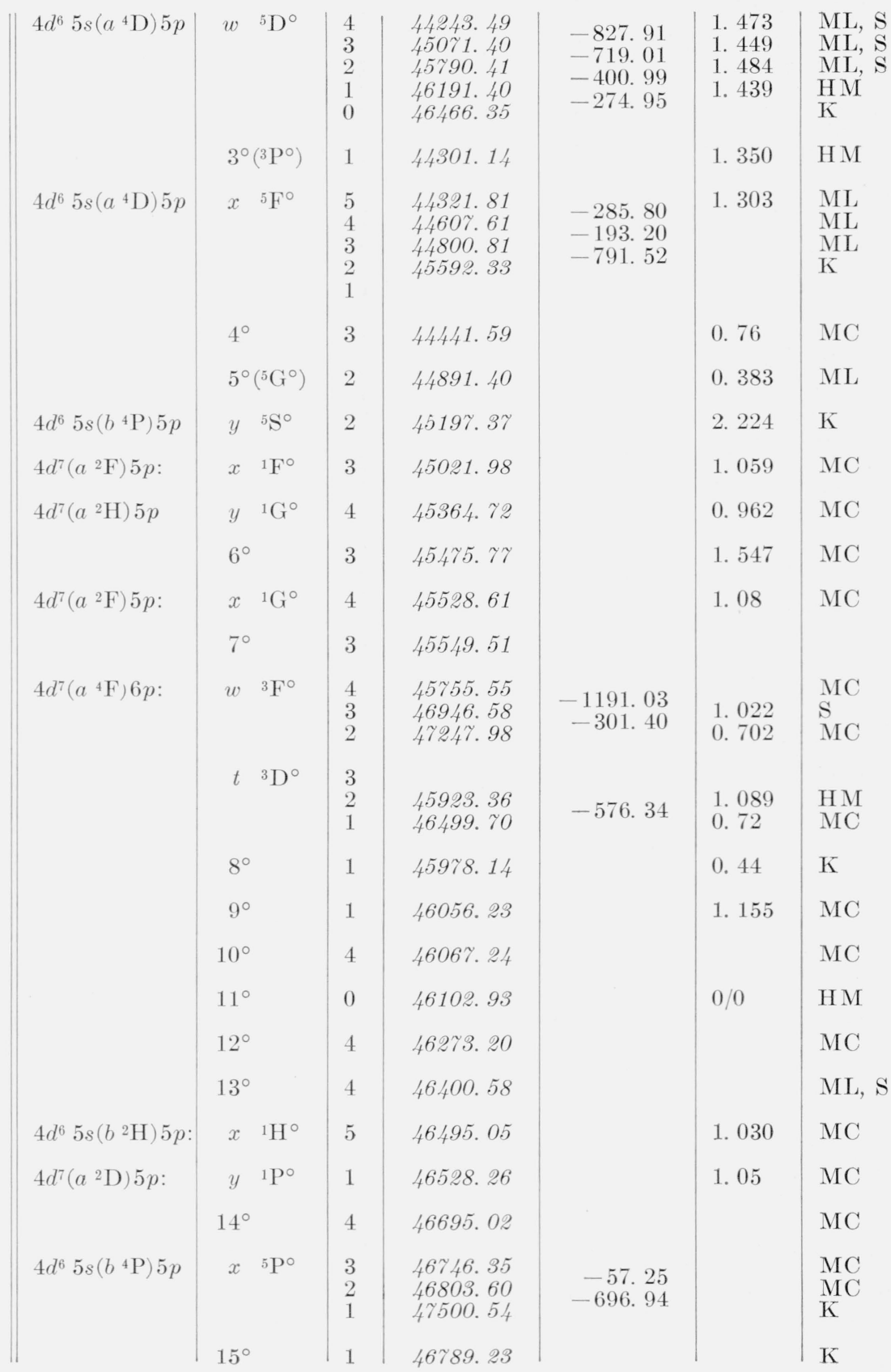


TABLe 1-Continued

\begin{tabular}{|c|c|c|c|c|c|c|c|c|c|c|c|c|c|}
\hline Config. & Desig. & $J$ & Level & Interval & Obs. $g$ & $\begin{array}{l}\text { Earliest } \\
\text { reference }\end{array}$ & Config. & Desig. & $J$ & Level & Interval & Obs. $g$ & $\begin{array}{l}\text { Earliest } \\
\text { reference }\end{array}$ \\
\hline $4 d^{7}\left(a^{4} \mathrm{~F}\right) 5 d$ & $f \quad{ }^{5} \mathrm{~F}$ & $\begin{array}{l}5 \\
4 \\
3 \\
2 \\
1\end{array}$ & $\begin{array}{l}\text { 46906. } 32 \\
\text { 48543. } 88 \\
\text { 48809. } 28\end{array}$ & $\begin{array}{r}-1637.56 \\
-265.40\end{array}$ & & $\begin{array}{l}\mathrm{S} \\
\mathrm{K} \\
\mathrm{K}\end{array}$ & & $\begin{array}{l}28^{\circ} \\
29^{\circ} \\
30^{\circ}\end{array}$ & $\begin{array}{l}3 \\
3 \\
4\end{array}$ & $\begin{array}{l}47868.35 \\
48003.00 \\
48109.32\end{array}$ & & 1. 309 & $\begin{array}{l}\mathrm{MC} \\
\mathrm{MC} \\
\mathrm{MC}\end{array}$ \\
\hline $4 d^{7}\left(a^{4} \mathrm{~F}\right) 5 d:$ & $e^{5} \mathrm{D}$ & $\begin{array}{l}4 \\
3 \\
2 \\
1 \\
0\end{array}$ & $\begin{array}{l}\text { 46972. } 42 \\
47188.32 \\
48720.87 \\
49513.33 ?\end{array}$ & $\begin{array}{r}-215.90 \\
-1532.55 \\
-792.46 ?\end{array}$ & 1. 36 & $\begin{array}{l}\mathrm{K} \\
\mathrm{MC} \\
\mathrm{S} \\
\mathrm{MC}\end{array}$ & & $\begin{array}{l}31^{\circ} \\
32^{\circ} \\
33^{\circ}\end{array}$ & $\begin{array}{l}4 \\
2 \\
2\end{array}$ & $\begin{array}{l}48143.98 \\
48164.79 \\
48326.73\end{array}$ & & 1. 08 & $\begin{array}{l}\mathrm{MC} \\
\mathrm{MC} \\
\mathrm{K}\end{array}$ \\
\hline $4 \mathrm{~d}^{7}\left(a^{4} \mathrm{~F}\right) 5 d:$ & $e^{5} \mathrm{G}$ & $\begin{array}{l}6 \\
5 \\
4 \\
3 \\
2\end{array}$ & $\begin{array}{l}\text { 46991. } 15 \\
\text { 48521. } 77 \\
\text { 49604. } 93 \\
\text { 49553. } 73\end{array}$ & $\begin{array}{r}-1530.62 \\
-51.20\end{array}$ & 1. $35 ?$ & $\begin{array}{l}\mathrm{K} \\
\mathrm{K} \\
\mathrm{K} \\
\mathrm{MC}\end{array}$ & $4 d^{6} 5 s\left(a^{6} \mathrm{D}\right) 6 s$ & $e^{7 \mathrm{D}}$ & $\begin{array}{l}5 \\
4 \\
3 \\
2 \\
1\end{array}$ & $\begin{array}{l}48386.33 \\
49235.15 \\
50016.70 \\
50539.81 \\
50618.88\end{array}$ & $\begin{array}{r}-848.82 \\
-781.55 \\
-523.11 \\
-79.09\end{array}$ & $\begin{array}{l}\text { 1. } 602 \\
\text { 1. } 649\end{array}$ & $\begin{array}{l}\mathrm{K} \\
\mathrm{K} \\
\mathrm{K} \\
\mathrm{K} \\
\mathrm{K}\end{array}$ \\
\hline & $16^{\circ}$ & 3 & 47046.54 & & 1. 058 & $\mathrm{MC}$ & & $34^{\circ}$ & 3 & 48405.09 & & 1. 14 & $\mathrm{MC}$ \\
\hline $4 d^{7}\left(a^{4} \mathrm{~F}\right) 5 d:$ & $e^{3} \mathrm{G}$ & $\begin{array}{l}5 \\
4 \\
3\end{array}$ & $\begin{array}{l}47084.80 \\
48727.68 \\
49675.97\end{array}$ & $\begin{array}{r}-1642.88 \\
-948.29\end{array}$ & 1. 19 & $\begin{array}{l}\mathrm{S} \\
\mathrm{K} \\
\mathrm{K}\end{array}$ & $4 d^{7}\left(a^{4} \mathrm{~F}\right) 5 d:$ & $e{ }^{3} \mathrm{D}$ & $\begin{array}{l}3 \\
2 \\
1\end{array}$ & $\begin{array}{l}\text { 48489. } 66 \\
49757.88\end{array}$ & -1268.22 & 1. 28 & $\begin{array}{l}\mathrm{K} \\
\mathrm{MC}\end{array}$ \\
\hline & $17^{\circ}$ & 4 & $4715 \% .28$ & & 1. 24 & $\mathrm{MC}$ & & $35^{\circ}$ & 3 & 48493.01 & & 1. $14 ?$ & $\mathrm{MC}$ \\
\hline & $18^{\circ}\left({ }^{5} \mathrm{D}^{\circ}\right)$ & 0 & 47176.90 & & & K & & $36^{\circ}$ & 5 & 48503.30 & & & $\mathrm{MC}$ \\
\hline & $19^{\circ}$ & 4 & 47261.52 & & 1. 12 & $\mathrm{MC}$ & & $37^{\circ}$ & $3 ?$ & 48570.85 & & & $\mathrm{MC}$ \\
\hline $4 d^{7}\left(a^{4} \mathrm{~F}\right) 6 p:$ & $s \quad{ }^{3} \mathrm{D}^{\circ}$ & $\begin{array}{l}3 \\
2 \\
1\end{array}$ & $\begin{array}{l}47339.32 \\
4754 \% .33\end{array}$ & -208.01 & $\begin{array}{l}\text { 1. } 377 \\
\text { 1. } 057\end{array}$ & $\begin{array}{l}\mathrm{MC} \\
\mathrm{MC}\end{array}$ & & $\begin{array}{l}38^{\circ} \\
39^{\circ}\end{array}$ & $\begin{array}{l}4 \\
1\end{array}$ & $\begin{array}{l}48597.45 \\
48604.34\end{array}$ & & $\begin{array}{l}\text { 1. } 208 \\
\text { 2. } 06\end{array}$ & $\begin{array}{l}\mathrm{MC} \\
\mathrm{MC}\end{array}$ \\
\hline & $20^{\circ}\left({ }^{5} \mathrm{D}^{\circ}\right)$ & 2 & 47345.10 & & 1. 55 & $\mathrm{MC}$ & & $40^{\circ}$ & 3 & 48765.88 & & & $\mathrm{MC}$ \\
\hline $4 d^{7}\left(a^{4} \mathrm{~F}\right) 5 p:$ & $e{ }^{3} \mathrm{P}$ & 2 & 47458. 76 & & & & & $41^{\circ}$ & 2 & 48779.15 & & & $\mathrm{MC}$ \\
\hline & $21^{\circ}$ & 5 & 47425.17 & & & K & & $42^{\circ}$ & 4 & 48853.69 & & 1. 23 & K \\
\hline & $\mathrm{B}\left({ }^{3} \mathrm{~F}\right)$ & 4 & 47486.96 & & & S & & $43^{\circ}$ & 3 & 48933.93 & & & $\mathrm{MC}$ \\
\hline & $22^{\circ}$ & 3 & 47526.09 & & 1. 134 & $\mathrm{MC}$ & & $44^{\circ}$ & 2 & $4903 \% .35$ & & & $\mathrm{~K}$ \\
\hline & $23^{\circ}$ & 3 & 47635.33 & & 1. 06 & $\mathrm{MC}$ & & $45^{\circ}$ & 1 & 49047.61 & & & $\mathrm{MC}$ \\
\hline & $24^{\circ}$ & 5 & 47642.87 & & & K & & $46^{\circ}$ & 3 & 49141.42 & & 1. $03 ?$ & $\mathrm{MC}$ \\
\hline & $25^{\circ}$ & 3 & 47788.72 & & & $\mathrm{MC}$ & & $47^{\circ}$ & 4 & 49165.05 & & & $\mathrm{MC}$ \\
\hline & $\begin{array}{l}26^{\circ} \\
27^{\circ}\end{array}$ & $\begin{array}{l}1 \\
4\end{array}$ & $\begin{array}{l}47809.11 \\
4781 \% .84\end{array}$ & & 1. $32 ?$ & $\begin{array}{l}\mathrm{MC} \\
\mathrm{MC}\end{array}$ & $4 d^{7}\left(a^{4} \mathrm{P}\right) 5 d$ & $e{ }^{5} \mathrm{P}$ & $\begin{array}{l}3 \\
2 \\
1\end{array}$ & $\begin{array}{l}\text { 49291. } 06 \\
50172.84\end{array}$ & -881.78 & & $\begin{array}{l}K \\
K\end{array}$ \\
\hline
\end{tabular}




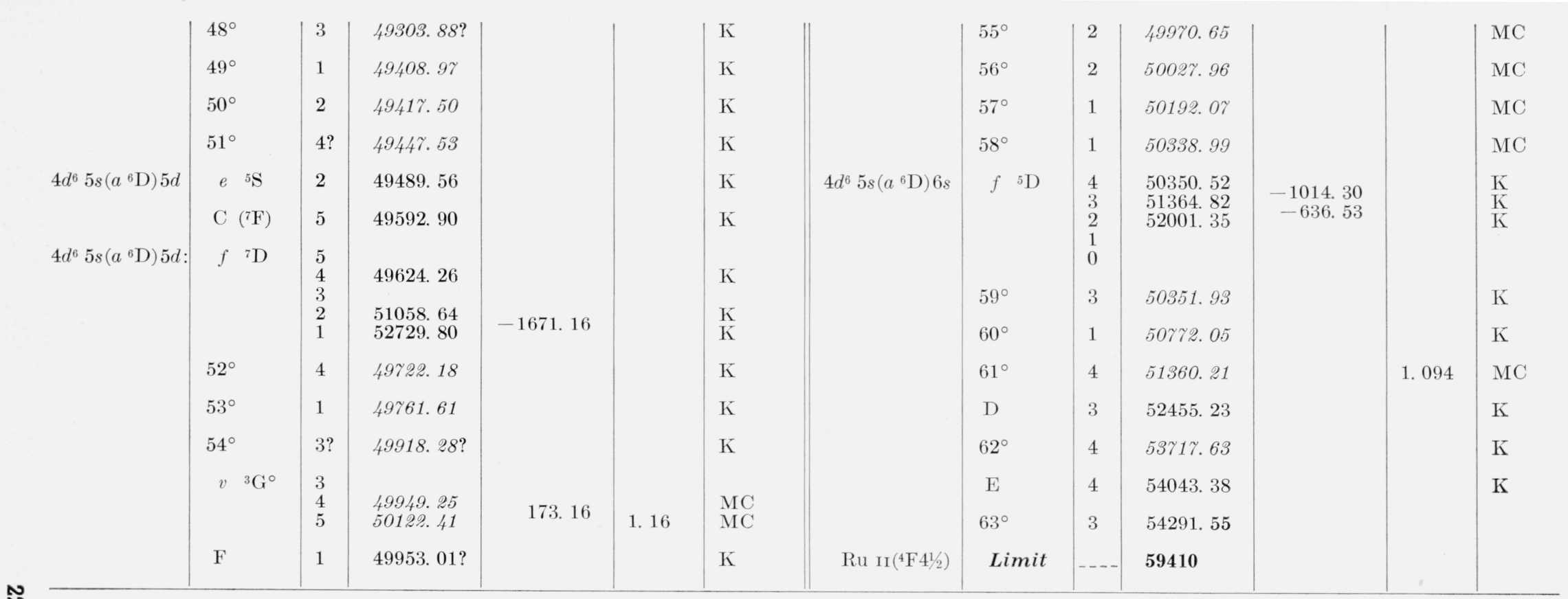

N 
TABLE 2. Ru I observed terms*

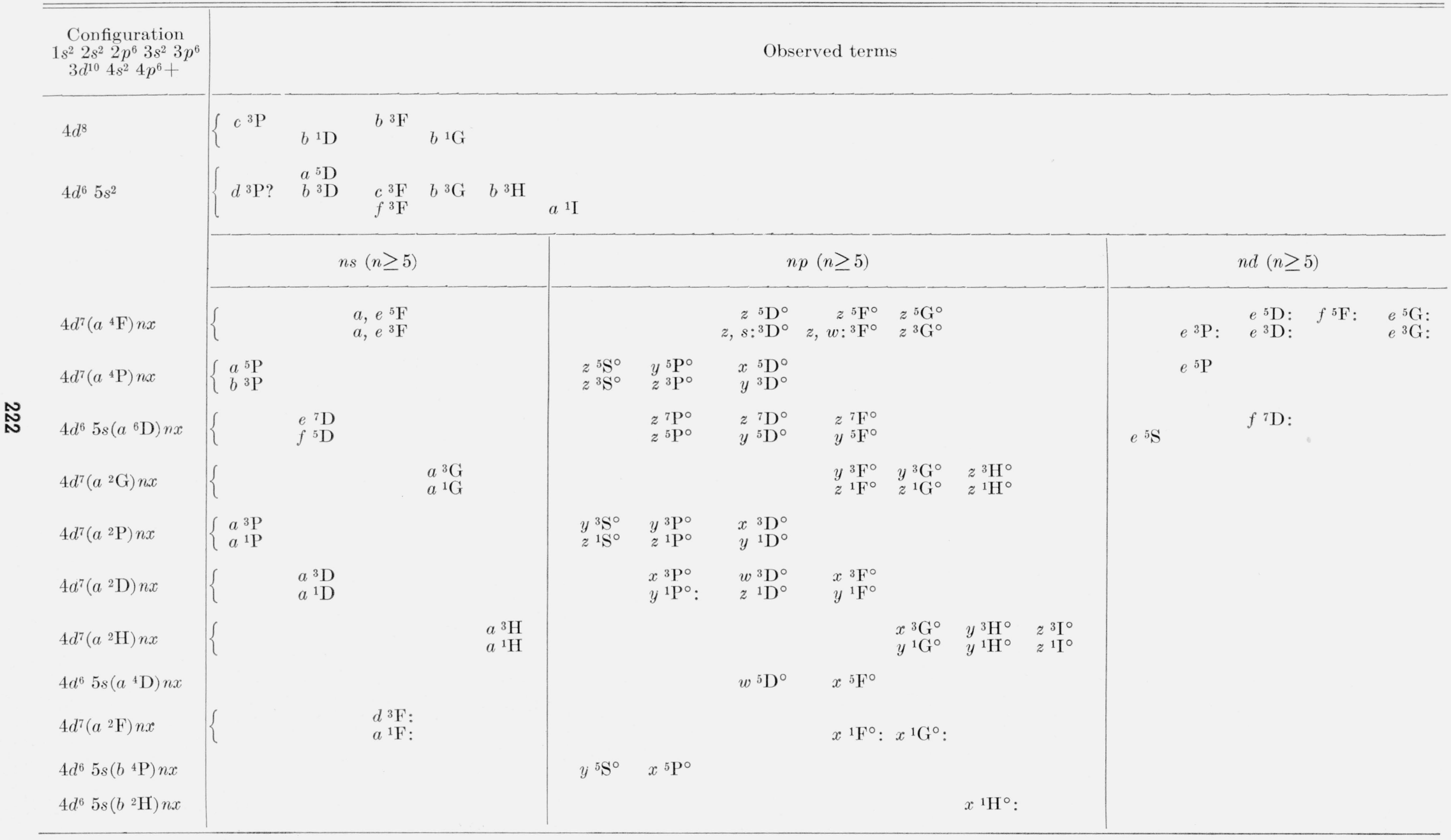

*For predicted terms in the spectra of the $\mathrm{Ru} \mathrm{I}$ isoelectronic sequence, see Vol. III, Introduction. 
Table 3

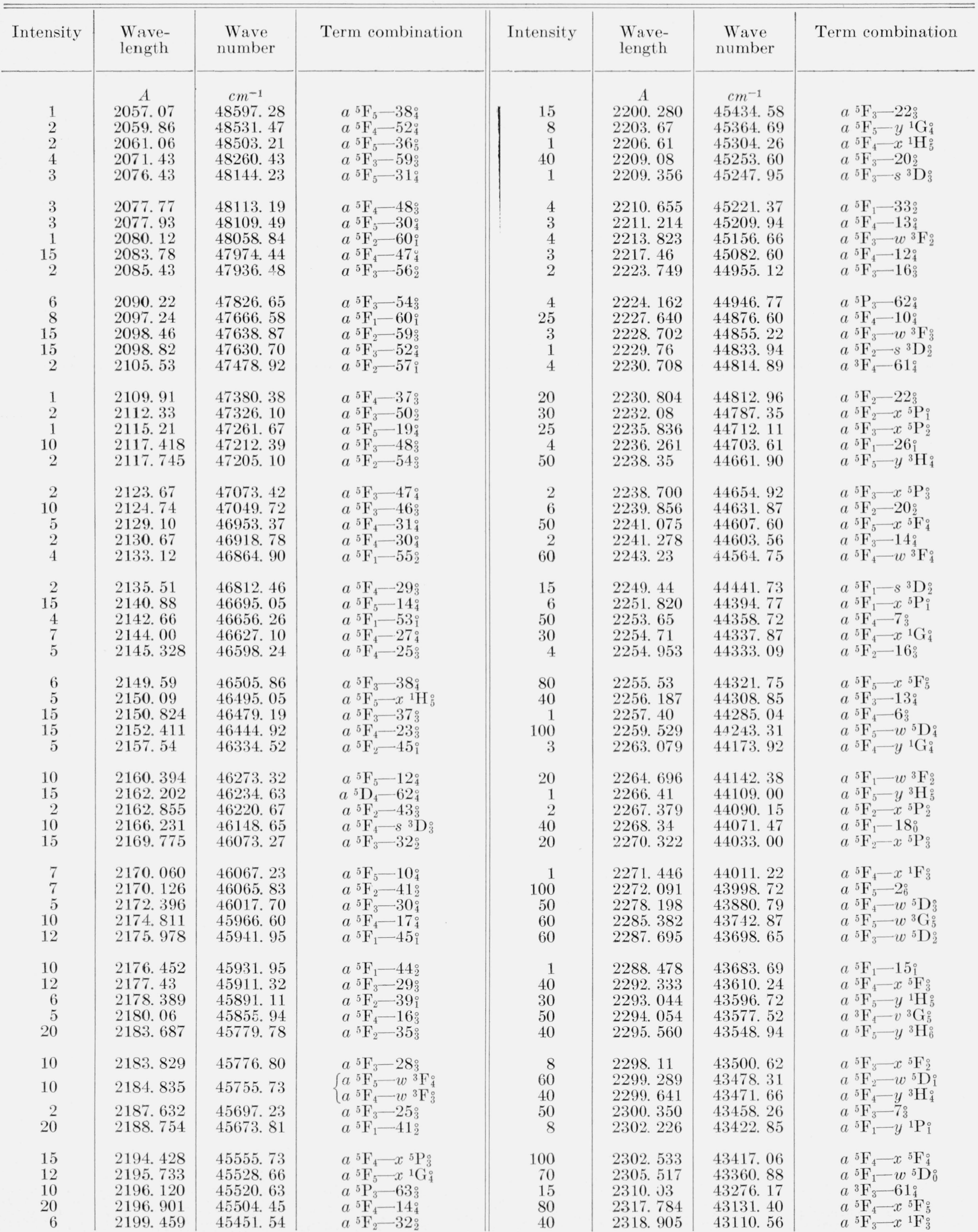


TABLE 3-Continued

\begin{tabular}{|c|c|c|c|c|c|c|c|}
\hline Intensity & $\begin{array}{l}\text { Wave- } \\
\text { length }\end{array}$ & $\begin{array}{c}\text { Wave } \\
\text { number }\end{array}$ & Term combination & Intensity & $\begin{array}{l}\text { Wave- } \\
\text { length }\end{array}$ & $\begin{array}{c}\text { Wave } \\
\text { number }\end{array}$ & Term combination \\
\hline $\begin{array}{l}40 \\
60 \\
80 \\
60 \\
15\end{array}$ & $\begin{array}{c}\text { A } \\
2320.23 \\
2320.699 \\
2322.009 \\
2325.952 \\
2327.536\end{array}$ & $\begin{array}{c}\mathrm{cm}^{-1} \\
43085.94 \\
43077.23 \\
43052.93 \\
42979.95 \\
42950.71\end{array}$ & $\begin{array}{l}a^{5} \mathrm{~F}_{1}-w^{5} \mathrm{D}_{1}^{\circ} \\
a^{5} \mathrm{~F}_{2}-w^{5} \mathrm{D}_{2}^{\circ} \\
a^{5} \mathrm{~F}_{4}-w^{5} \mathrm{D}_{4}^{\circ} \\
a^{5} \mathrm{~F}_{3}-w^{5} \mathrm{D}_{3}^{\circ} \\
a^{5} \mathrm{~F}_{1}-9_{1}^{\circ}\end{array}$ & $\begin{array}{r}1 \\
15 \\
5 \\
50 \\
2\end{array}$ & $\begin{array}{c}A \\
2421.580 \\
2421.772 \\
2422.165 \\
2422.574 \\
2422.767\end{array}$ & $\begin{array}{c}\mathrm{cm}^{-1} \\
41282.82 \\
41279.54 \\
41272.85 \\
41265.88 \\
41262.59\end{array}$ & $\begin{array}{ll}a & { }^{5} \mathrm{D}_{4}-40_{3}^{\circ} \\
a & { }^{5} \mathrm{D}_{0}-60_{1}^{\circ} \\
a & { }^{3} \mathrm{~F}_{4}-27_{4}^{\circ} \\
a & { }^{5} \mathrm{D}_{1}-58_{1}^{\circ} \\
a^{5} \mathrm{~F}_{2}-x^{3} \mathrm{G}_{3}^{\circ}\end{array}$ \\
\hline $\begin{array}{r}2 \\
60 \\
40 \\
1 \\
15\end{array}$ & $\begin{array}{l}2329.30 \\
2331.43 \\
2331.770 \\
2331.98 \\
2333.736\end{array}$ & $\begin{array}{l}\text { 42918. } 18 \\
42878.98 \\
42872.72 \\
42868.86 \\
42836.67\end{array}$ & $\begin{array}{l}a{ }^{5} \mathrm{~F}_{4}-y{ }^{3} \mathrm{H}_{5}^{\circ} \\
a^{5} \mathrm{~F}_{2}-x{ }^{5} \mathrm{~F}_{2}^{\circ} \\
a^{5} \mathrm{~F}_{1}-8_{1}^{\circ} \\
a^{5} \mathrm{D}_{4}-59_{3}^{\circ} \\
a^{5} \mathrm{~F}_{2}-7_{3}^{\circ}\end{array}$ & $\begin{array}{r}4 \\
30 \\
5 \\
10 \\
10\end{array}$ & $\begin{array}{l}2423.089 \\
2423.877 \\
2425.299 \\
2426.699 \\
2428.323\end{array}$ & $\begin{array}{l}41257.11 \\
41243.69 \\
41219.52 \\
41195.74 \\
41168.19\end{array}$ & $\begin{array}{ll}a & { }^{5} \mathrm{P}_{3}-56_{2}^{\circ} \\
a & { }^{3} \mathrm{~F}_{4}-25_{3}^{\circ} \\
a & { }^{3} \mathrm{~F}_{3}-48_{3}^{\circ} \\
a & { }^{5} \mathrm{~F}_{1}-3{ }_{1}^{\circ} \\
a & { }^{3} \mathrm{~F}_{2}-59_{3}^{\circ}\end{array}$ \\
\hline $\begin{array}{r}40 \\
1 \\
6 \\
100 \\
40\end{array}$ & $\begin{array}{l}2335.738 \\
2336.53 \\
2337.98 \\
2340.696 \\
2342.02\end{array}$ & $\begin{array}{l}42799.90 \\
42785.39 \\
42758.86 \\
42709.25 \\
42685.11\end{array}$ & $\begin{array}{l}a^{5} \mathrm{~F}_{3}-5_{2}^{\circ} \\
a^{5} \mathrm{~F}_{4}-x^{3} \mathrm{G}_{3}^{\circ} \\
a^{3} \mathrm{~F}_{4}-48_{3}^{\circ} \\
a^{5} \mathrm{~F}_{3}-x^{5} \mathrm{~F}_{3}^{\circ} \\
a^{5} \mathrm{~F}_{1}-w^{5} \mathrm{D}_{2}^{\circ}\end{array}$ & $\begin{array}{r}80 \\
5 \\
40 \\
90 \\
20\end{array}$ & $\begin{array}{l}2429.594 \\
2430.62 \\
2431.51 \\
2432.915 \\
2433.064\end{array}$ & $\begin{array}{l}41146.65 \\
41129.28 \\
41114.23 \\
41090.49 \\
41087.97\end{array}$ & $\begin{array}{l}a{ }^{5} \mathrm{D}_{3}-52_{4}^{\circ} \\
a^{5} \mathrm{~F}_{1}-x^{3} \mathrm{P}_{2}^{\circ} \\
a^{5} \mathrm{D}_{4}-38_{4}^{\circ} \\
a^{3} \mathrm{~F}_{4}-23_{3}^{\circ} \\
a^{5} \mathrm{D}_{4}-37_{3}^{\circ}\end{array}$ \\
\hline $\begin{array}{r}80 \\
8 \\
100 \\
150 \\
200\end{array}$ & $\begin{array}{l}2342.72 \\
2344.534 \\
2348.318 \\
2349.338 \\
2351.33\end{array}$ & $\begin{array}{l}42672.35 \\
42639.34 \\
42570.64 \\
42552.16 \\
42516.11\end{array}$ & $\begin{array}{l}a^{5} \mathrm{~F}_{4}-w^{3} \mathrm{G}_{4}^{\circ} \\
a^{5} \mathrm{D}_{4}-v^{3} \mathrm{G}_{5}^{\circ} \\
a^{5} \mathrm{~F}_{3}-y^{3} \mathrm{H}_{4}^{\circ} \\
a^{5} \mathrm{~F}_{4}-w^{3} \mathrm{G}_{5}^{\circ} \\
a^{5} \mathrm{~F}_{3}-x^{5} \mathrm{~F}_{4}^{\circ}\end{array}$ & $\begin{array}{r}25 \\
40 \\
2 \\
50 \\
6\end{array}$ & $\begin{array}{l}2433.477 \\
2434.879 \\
2437.078 \\
2437.790 \\
2438.055\end{array}$ & $\begin{array}{l}41081.00 \\
41057.35 \\
41020.30 \\
41008.33 \\
41003.87\end{array}$ & $\begin{array}{l}a^{3} \mathrm{~F}_{3}-47_{4}^{\circ} \\
a^{3} \mathrm{~F}_{3}-46_{3}^{\circ} \\
a^{5} \mathrm{D}_{4}-36_{5}^{\circ} \\
a^{3} \mathrm{~F}_{2}-57_{1}^{\circ} \\
a^{5} \mathrm{P}_{2}-45_{1}^{\circ}\end{array}$ \\
\hline $\begin{array}{r}6 \\
15 \\
6 \\
90 \\
50\end{array}$ & $\begin{array}{l}2352.84 \\
2352.94 \\
2354.10 \\
2360.093 \\
2360.712\end{array}$ & $\begin{array}{l}42488.83 \\
42487.02 \\
42466.08 \\
42358.26 \\
42347.16\end{array}$ & $\begin{array}{l}a{ }^{5} \mathrm{~F}_{2}-x{ }^{1} \mathrm{~F}_{3}^{\circ} \\
a^{5} \mathrm{~F}_{1}-x{ }^{5} \mathrm{~F}_{2}^{\circ} \\
a^{5} \mathrm{D}_{4}-v^{3} \mathrm{G}_{4}^{\circ} \\
a^{5} \mathrm{~F}_{2}-w^{5} \mathrm{D}_{3}^{\circ} \\
a^{5} \mathrm{~F}_{5}-x^{3} \mathrm{~F}_{4}^{\circ}\end{array}$ & $\begin{array}{r}1 \\
20 \\
20 \\
7 \\
50\end{array}$ & $\begin{array}{l}2439.408 \\
2440.05 \\
2440.977 \\
2441.200 \\
2442.934\end{array}$ & $\begin{array}{l}40981.13 \\
40970.35 \\
40954.79 \\
40951.05 \\
40921.98\end{array}$ & $\begin{array}{l}a^{3} \mathrm{~F}_{4}-22_{3}^{\circ} \\
a^{5} \mathrm{D}_{2}-56_{2}^{\circ} \\
a^{5} \mathrm{D}_{1}-56_{2}^{\circ} \\
a^{5} \mathrm{P}_{3}-52_{4}^{\circ} \\
a^{5} \mathrm{D}_{4}-34_{3}^{\circ}\end{array}$ \\
\hline $\begin{array}{r}2 \\
1 \\
25 \\
10 \\
80\end{array}$ & $\begin{array}{l}2362.871 \\
2365.14 \\
2366.743 \\
2367.769 \\
2370.169\end{array}$ & $\begin{array}{l}42308.46 \\
42267.88 \\
42239.26 \\
42220.95 \\
42178.20\end{array}$ & $\begin{array}{l}a^{3} \mathrm{~F}_{4}-42_{4}^{\circ} \\
a^{3} \mathrm{~F}_{3}-59_{3}^{\circ} \\
a^{5} \mathrm{D}_{4}-52_{4}^{\circ} \\
a^{3} \mathrm{~F}_{4}-40_{3}^{\circ} \\
a^{5} \mathrm{~F}_{2}-5_{2}^{\circ}\end{array}$ & $\begin{array}{r}1 \\
1 \\
1 \\
60 \\
30\end{array}$ & $\begin{array}{l}2443.46 \\
2443.86 \\
2444.189 \\
2444.38 \\
2444.828\end{array}$ & $\begin{array}{l}\text { 40913. } 17 \\
40906.48 \\
40900.97 \\
40897.78 \\
40890.28\end{array}$ & $\begin{array}{l}a{ }^{5} \mathrm{D}_{2}-55_{2}^{\circ} \\
a^{5} \mathrm{~F}_{3}-y^{1} \mathrm{~F}_{3}^{\circ} \\
a^{3} \mathrm{G}_{4}-62_{4}^{\circ} \\
a^{5} \mathrm{D}_{1}-55_{2}^{\circ} \\
a^{5} \mathrm{P}_{2}-43_{3}^{\circ}\end{array}$ \\
\hline $\begin{array}{r}3 \\
100 \\
2 \\
6 \\
2\end{array}$ & $\begin{array}{l}2372.13 \\
2375.272 \\
2378.75 \\
2386.678 \\
2387.359\end{array}$ & $\begin{array}{l}42143.34 \\
42087.60 \\
42026.06 \\
41886.47 \\
41874.53\end{array}$ & $\begin{array}{l}a{ }^{5} \mathrm{~F}_{3}-x{ }^{3} \mathrm{P}_{2}^{\circ} \\
a^{5} \mathrm{~F}_{2}-x{ }^{5} \mathrm{~F}_{3}^{\circ} \\
a^{3} \mathrm{~F}_{4}-37_{3}^{\circ} \\
a^{3} \mathrm{~F}_{3}-55_{2}^{\circ} \\
a^{5} \mathrm{P}_{2}-54_{3}^{\circ}\end{array}$ & $\begin{array}{r}50 \\
2 \\
2 \\
40 \\
10\end{array}$ & $\begin{array}{l}2445.43 \\
2446.600 \\
2447.25 \\
2447.439 \\
2447.566\end{array}$ & $\begin{array}{l}40880.22 \\
40860.67 \\
40849.82 \\
40846.67 \\
40844.54\end{array}$ & $\begin{array}{l}a^{3} \mathrm{~F}_{4}-21_{5}^{\circ} \\
a^{5} \mathrm{D}_{2}-54_{3}^{\circ} \\
a^{3} \mathrm{~F}_{3}-43_{3}^{\circ} \\
a^{5} \mathrm{D}_{0}-58_{1}^{\circ} \\
a^{3} \mathrm{~F}_{2}-56_{2}^{\circ}\end{array}$ \\
\hline $\begin{array}{r}100 \\
15 \\
1 \\
90 \\
150\end{array}$ & $\begin{array}{l}\text { 2387. } 881 \\
2388.188 \\
2390.423 \\
2392.249 \\
2392.425\end{array}$ & $\begin{array}{l}41865.37 \\
41859.99 \\
41820.86 \\
41771.48 \\
41785.86\end{array}$ & $\begin{array}{l}a{ }^{3} \mathrm{~F}_{3}-v^{3} \mathrm{G}_{4} \\
a^{3} \mathrm{~F}_{4}-34_{3}^{\circ} \\
a^{5} \mathrm{D}_{4}-48_{3}^{\circ} \\
a^{5} \mathrm{~F}_{3}-w^{3} \mathrm{G}_{4}^{\circ} \\
a^{5} \mathrm{~F}_{1}-5_{2}^{\circ}\end{array}$ & $\begin{array}{r}2 \\
30 \\
100 \\
2 \\
8\end{array}$ & $\begin{array}{l}2448.512 \\
2450.359 \\
2450.560 \\
2452.06 \\
2454.555\end{array}$ & $\begin{array}{l}40828.76 \\
40797.99 \\
40794.64 \\
40769.69 \\
40728.25\end{array}$ & $\begin{array}{l}b^{3} \mathrm{~F}_{4}-v^{3} \mathrm{G}_{4} \\
a{ }^{5} \mathbf{F}_{1}-y^{1} \mathrm{D}_{2}^{\circ} \\
a^{3} \mathbf{F}_{4}-s^{3} \mathrm{D}_{3}^{\circ} \\
a^{3} \mathbf{F}_{3}-42_{4}^{\circ} \\
a^{5} \mathrm{D}_{3}-48_{3}^{\circ}\end{array}$ \\
\hline $\begin{array}{r}60 \\
80 \\
3 \\
20 \\
20\end{array}$ & $\begin{array}{l}2392.963 \\
2395.721 \\
2396.32 \\
\text { 2397. } 406 \\
2398.38\end{array}$ & $\begin{array}{l}41776.47 \\
41728.38 \\
41717.95 \\
41699.05 \\
41682.12\end{array}$ & $\begin{array}{l}a^{5} \mathrm{D}_{3}-59_{3}^{\circ} \\
a^{5} \mathrm{~F}_{2}-4_{3}^{\circ} \\
a^{5} \mathrm{P}_{2}-53_{1}^{\circ} \\
a^{5} \mathrm{D}_{1}-60_{1}^{\circ} \\
a^{5} \mathrm{D}_{4}-47_{4}^{\circ}\end{array}$ & $\begin{array}{r}150 \\
30 \\
10 \\
100 \\
30\end{array}$ & $\begin{array}{l}2454.926 \\
2456.279 \\
2456.946 \\
2458.622 \\
2460.73\end{array}$ & $\begin{array}{l}40722.10 \\
40699.67 \\
40688.62 \\
40660.89 \\
40626.06\end{array}$ & $\begin{array}{l}a{ }^{5} \mathrm{P}_{2}-40_{3}^{\circ} \\
a^{5} \mathrm{D}_{0}-57_{1}^{\circ} \\
a^{5} \mathrm{D}_{1}-53_{1}^{\circ} \\
a^{5} \mathrm{D}_{4}-31_{4}^{\circ} \\
a^{5} \mathrm{D}_{4}-30_{4}^{\circ}\end{array}$ \\
\hline $\begin{array}{r}80 \\
25 \\
3 \\
1 \\
50\end{array}$ & $\begin{array}{l}\text { 2399. } 750 \\
2403.173 \\
2403.798 \\
2404.207 \\
2405.180\end{array}$ & $\begin{array}{l}41658.33 \\
41598.99 \\
41588.18 \\
41581.10 \\
41564.28\end{array}$ & $\begin{array}{l}a{ }^{5} \mathrm{D}_{4}-46_{3}^{\circ} \\
a^{3} \mathrm{~F}_{4}-31_{4}^{\circ} \\
a^{3} \mathrm{~F}_{2}-60_{1}^{\circ} \\
a^{5} \mathrm{P}_{3}-59_{3}^{\circ} \\
a^{3} \mathrm{~F}_{4}-30_{4}^{\circ}\end{array}$ & $\begin{array}{r}100 \\
20 \\
100 \\
40 \\
3\end{array}$ & $\begin{array}{l}2462.943 \\
2464.366 \\
2464.699 \\
2467.576 \\
2468.29\end{array}$ & $\begin{array}{l}40589.56 \\
40566.12 \\
40560.64 \\
40513.35 \\
40501.63\end{array}$ & 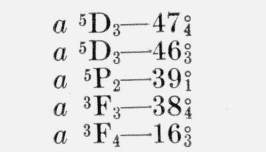 \\
\hline $\begin{array}{r}10 \\
60 \\
10 \\
20 \\
4\end{array}$ & $\begin{array}{l}2407.66 \\
2408.290 \\
2411.76 \\
2415.005 \\
2416.247\end{array}$ & $\begin{array}{l}41521.47 \\
41510.61 \\
41450.89 \\
41395.20 \\
41373.92\end{array}$ & $\begin{array}{l}a{ }^{5} \mathrm{~F}_{2}-x^{3} \mathrm{P}_{2}^{\circ} \\
a^{3} \mathrm{G}_{5}-62_{4}^{\circ} \\
a{ }^{5} \mathrm{D}_{4}-43_{3}^{\circ} \\
a^{5} \mathrm{D}_{3}-55_{2}^{\circ} \\
a^{5} \mathrm{D}_{3}-v^{3} \mathrm{G}_{4}^{\circ}\end{array}$ & $\begin{array}{r}2 \\
30 \\
50 \\
40 \\
80\end{array}$ & $\begin{array}{l}2469.20 \\
2470.71 \\
2471.48 \\
2472.09 \\
2474.029\end{array}$ & $\begin{array}{l}40486.71 \\
40461.96 \\
40449.36 \\
40439.38 \\
40407.69\end{array}$ & $\begin{array}{ll}a & { }^{3} \mathrm{~F}_{3}-37_{3}^{\circ} \\
a & { }^{5} \mathrm{D}_{3}-44_{2}^{\circ} \\
a & { }^{5} \mathrm{P}_{2}-35_{3}^{\circ} \\
a & { }^{5} \mathrm{~F}_{5}-1_{4}^{\circ} \\
a^{5} \mathrm{P}_{1}-56_{2}^{\circ}\end{array}$ \\
\hline $\begin{array}{r}4 \\
5 \\
4 \\
2 \\
30\end{array}$ & $\begin{array}{l}\text { 2416. } 443 \\
2416.754 \\
2418.065 \\
2418.607 \\
2419.205\end{array}$ & $\begin{array}{l}41370.57 \\
41365.24 \\
41342.82 \\
41333.56 \\
41323.34\end{array}$ & $\begin{array}{l}a{ }^{5} \mathrm{D}_{4}-42_{4}^{\circ} \\
a \\
{ }^{5} \mathrm{P}_{2}-49_{1}^{\circ} \\
a \\
{ }^{5} \mathrm{D}_{3}-54_{3}^{\circ} \\
a^{3} \mathrm{~F}_{3}-50_{2}^{\circ} \\
a^{3} \mathrm{~F}_{4}-28_{3}^{\circ}\end{array}$ & $\begin{array}{c}15 \\
30 \\
200 \mathrm{H} \\
50 \\
100\end{array}$ & $\begin{array}{l}2474.396 \\
2474.846 \\
2475.395 \\
2476.32 \\
2476.869\end{array}$ & $\begin{array}{l}40401.69 \\
40394.35 \\
40385.39 \\
40370.31 \\
40361.36\end{array}$ & $\begin{array}{ll}a & { }^{3} \mathrm{~F}_{4}-w^{3} \mathrm{~F}_{3}^{\circ} \\
a & { }^{5} \mathrm{~F}_{2}-y^{3} \mathrm{~S}_{1}^{\circ} \\
a & { }^{5} \mathrm{D}_{4}-28_{3}^{\circ} \\
a & { }^{5} \mathrm{P}_{3}-46_{3}^{\circ} \\
a & { }^{5} \mathrm{P}_{2}-34_{3}^{\circ}\end{array}$ \\
\hline
\end{tabular}


TABle 3 -Continued

\begin{tabular}{|c|c|c|c|c|c|c|c|}
\hline Intensity & $\begin{array}{l}\text { Wave- } \\
\text { length }\end{array}$ & $\begin{array}{l}\text { Wave } \\
\text { number }\end{array}$ & Term combination & Intensity & $\begin{array}{l}\text { Wave- } \\
\text { length }\end{array}$ & $\begin{array}{l}\text { Wave } \\
\text { number }\end{array}$ & Term combination \\
\hline $\begin{array}{r}1 \\
1 \\
20 \\
15 \\
2\end{array}$ & $\begin{array}{l}\text { A } \\
2477.53 \\
2477.910 \\
2479.353 \\
2480.298 \\
2481.56\end{array}$ & $\begin{array}{l}c m^{-1} \\
40350.59 \\
40344.40 \\
40320.93 \\
40305.57 \\
40285.07\end{array}$ & $\begin{array}{lc}a & { }^{5} \mathrm{P}_{1}-55_{2}^{\circ} \\
a & { }^{5} \mathrm{D}_{1}-50_{2}^{\circ} \\
a & { }^{3} \mathrm{~F}_{3}-34_{3}^{\circ} \\
a & { }^{5} \mathrm{D}_{4}-25_{3}^{\circ} \\
a & { }^{5} \mathrm{~F}_{2}-y^{1} \mathrm{P}_{3}^{\circ}\end{array}$ & $\begin{array}{r}100 \\
1 \\
7 \\
10 \\
20\end{array}$ & \begin{tabular}{l}
\multicolumn{1}{c}{$A$} \\
2533.23 \\
2533.65 \\
2534.605 \\
2535.03 \\
2535.966
\end{tabular} & $\begin{array}{c}c m^{-1} \\
39463.44 \\
39456.89 \\
39442.03 \\
39435.41 \\
39420.86\end{array}$ & $\begin{array}{l}a{ }^{5} \mathrm{D}_{4}-w^{3} \mathbf{F}_{3}^{\circ} \\
a{ }^{5} \mathrm{P}_{2}-x{ }^{5} \mathrm{P}_{\mathbf{i}} \\
a \\
{ }^{3} \mathbf{F}_{3}-22_{3}^{\circ} \\
a^{5} \mathrm{D}_{2}-35_{3}^{\circ} \\
a^{3} \mathbf{F}_{2}-39_{1}^{\circ}\end{array}$ \\
\hline $\begin{array}{r}3 \\
2 \\
10 \\
10 \\
3\end{array}$ & $\begin{array}{l}2481.68 \\
2481.981 \\
2482.077 \\
2482.538 \\
2486.58\end{array}$ & $\begin{array}{l}40283.12 \\
40278.24 \\
40276.68 \\
40269.20 \\
40203.75\end{array}$ & $\begin{array}{l}a{ }^{5} \mathrm{P}_{2}-33_{2}^{\circ} \\
a{ }^{5} \mathrm{D}_{3}-42_{4}^{\circ} \\
a \quad{ }^{5} \mathrm{~F}_{5}-y^{3} \mathrm{G}_{4}^{\circ} \\
a{ }^{5} \mathrm{D}_{0}-53_{1}^{\circ} \\
a^{5} \mathrm{D}_{3}-41_{2}^{\circ}\end{array}$ & $\begin{array}{r}20 \\
5 \\
10 \\
6 \\
25\end{array}$ & $\begin{array}{l}2536.20 \\
2537.697 \\
2537.88 \\
2538.42 \\
2539.09\end{array}$ & $\begin{array}{l}39417.22 \\
39393.97 \\
39391.13 \\
39382.75 \\
39372.36\end{array}$ & $\begin{array}{ll}a & { }^{5} \mathrm{P}_{1}-44_{2}^{\circ} \\
a & 5 \mathrm{P}_{3}-32_{2}^{\circ} \\
a & \mathrm{~F}_{3}-w^{3} \mathrm{D}_{3}^{\circ} \\
b & { }^{3} \mathrm{~F}_{4}-36_{5}^{\circ} \\
b & { }^{3} \mathbf{F}_{4}-35_{3}^{\circ}\end{array}$ \\
\hline $\begin{array}{l}10 \\
30 \\
70 \\
20 \\
60\end{array}$ & $\begin{array}{l}2487.390 \\
2489.77 \\
2489.92 \\
2490.441 \\
2491.76\end{array}$ & $\begin{array}{l}40190.65 \\
40152.24 \\
40149.82 \\
40141.42 \\
40120.17\end{array}$ & $\begin{array}{ll}a & { }^{5} \mathrm{D}_{3}-40_{3}^{\circ} \\
a & 5 \mathrm{D}_{4}-23_{3}^{\circ} \\
a & { }^{3} \mathrm{~F}_{4}-14_{4}^{\circ} \\
a & { }^{5} \mathrm{P}_{1}-53_{1}^{\circ} \\
a^{3} \mathrm{~F}_{2}-48_{3}^{\circ}\end{array}$ & $\begin{array}{r}3 \\
70 \\
10 \\
50 \\
300\end{array}$ & $\begin{array}{l}2540.68 \\
2541.28 \\
2543.16 \\
2543.67 \\
2544.22\end{array}$ & $\begin{array}{l}39347.73 \\
39338.44 \\
39309.36 \\
39301.47 \\
39292.98\end{array}$ & $\begin{array}{l}a a^{5} \mathrm{D}_{2}-34_{3}^{\circ} \\
a \\
{ }^{5} \mathrm{P}_{3}-30_{4}^{\circ} \\
a^{3} \mathrm{~F}_{2}-35_{3}^{\circ} \\
a \\
{ }^{5} \mathrm{P}_{2}-20_{2}^{\circ} \\
a^{5} \mathrm{D}_{3}-28_{3}^{\circ}\end{array}$ \\
\hline $\begin{array}{r}80 \\
50 \\
40 \\
30 \\
2\end{array}$ & $\begin{array}{l}2494.022 \\
2496.56 \\
2497.680 \\
2497.866 \\
2499.550\end{array}$ & $\begin{array}{l}40083.79 \\
40043.04 \\
40025.09 \\
40022.11 \\
39995.15\end{array}$ & $\begin{array}{l}a{ }^{5} \mathrm{D}_{2}-46_{3}^{\circ} \\
a \\
{ }^{5} \mathrm{D}_{4}-22_{3}^{\circ} \\
a \\
{ }^{3} \mathrm{~F}_{3}-30_{4}^{\circ} \\
a \\
{ }^{5} \mathrm{D}_{3}-38_{4}^{\circ} \\
a^{5} \mathrm{P}_{3}-40_{3}^{\circ}\end{array}$ & $\begin{array}{r}1 \\
30 \\
15 \\
2 \\
150\end{array}$ & $\begin{array}{l}2545.48 \\
2545.76 \\
2546.14 \\
2546.285 \\
2546.668\end{array}$ & $\begin{array}{l}39273.53 \\
39269.21 \\
39263.35 \\
39261.12 \\
39255.21\end{array}$ & $\begin{array}{l}a{ }^{5} \mathrm{~F}_{5}-z{ }^{3} \mathrm{H}_{4}^{\circ} \\
a{ }^{5} \mathrm{D}_{2}-33_{2}^{\circ} \\
a^{5} \mathrm{D}_{4}-x{ }^{5} \mathrm{P}_{3}^{\circ} \\
a{ }^{3} \mathrm{~F}_{3}-20_{2}^{\circ} \\
a^{3} \mathbf{F}_{3}-s^{3} \mathrm{D}_{3}^{\circ}\end{array}$ \\
\hline $\begin{array}{r}8 \\
30 \\
70 \\
60 \\
25\end{array}$ & $\begin{array}{l}2500.518 \\
2500.835 \\
2501.48 \\
2501.885 \\
2502.37\end{array}$ & $\begin{array}{l}39979.66 \\
39974.60 \\
39964.29 \\
39957.82 \\
39950.08\end{array}$ & $\begin{array}{ll}a & 5 \mathrm{D}_{2}-44_{2}^{\circ} \\
a & { }^{5} \mathrm{D}_{1}-45_{1}^{\circ} \\
a & { }^{5} \mathrm{D}_{1}-44_{2}^{\circ} \\
a^{3} \mathbf{F}_{2}-46_{3}^{\circ} \\
a^{3} \mathbf{F}_{4}-x^{1} \mathrm{H}_{5}^{\circ}\end{array}$ & $\begin{array}{r}2 \\
20 \\
3 \\
4 \\
300\end{array}$ & $\begin{array}{l}2547.080 \\
2547.48 \\
2548.16 \\
2548.856 \\
2549.470\end{array}$ & $\begin{array}{l}39248.86 \\
39242.70 \\
39232.23 \\
39221.52 \\
39212.07\end{array}$ & $\begin{array}{ll}a & { }^{5} \mathrm{~F}_{4}-1_{4} \\
a & { }^{5} \mathrm{D}_{3}-27_{4} \\
a & { }^{5} \mathrm{P}_{3}-29_{3}^{\circ} \\
a^{3} \mathrm{~F}_{2}-34_{3}^{\circ} \\
a^{5} \mathrm{D}_{4}-14_{4}^{\circ}\end{array}$ \\
\hline $\begin{array}{r}15 \\
20 \\
20 \\
100 \\
30\end{array}$ & $\begin{array}{l}2502.87 \\
2504.40 \\
2504.51 \\
2508.270 \\
2510.13\end{array}$ & $\begin{array}{l}39942.09 \\
39917.70 \\
39915.94 \\
39856.11 \\
39826.58\end{array}$ & $\begin{array}{l}a \mathrm{D}_{4}-21_{5}^{\circ} \\
a^{5} \mathrm{D}_{3}-35_{3}^{\circ} \\
a \\
{ }^{5} \mathrm{~F}_{3}-x^{3} \mathrm{D}_{2}^{\circ} \\
a \\
{ }^{5} \mathrm{D}_{4}-s^{3} \mathrm{D}_{3}^{\circ} \\
a^{5} \mathrm{P}_{3}-38_{4}^{\circ}\end{array}$ & $\begin{array}{r}300 \\
30 \\
60 \\
25 \\
1\end{array}$ & $\begin{array}{l}2549.56 \\
2549.965 \\
2551.72 \\
2552.295 \\
2552.94\end{array}$ & $\begin{array}{l}39210.69 \\
39204.46 \\
39177.50 \\
39168.67 \\
39158.78\end{array}$ & $\begin{array}{ll}a & { }^{3} \mathrm{~F}_{3}-w^{3} \mathrm{~F}_{4}^{\circ} \\
a & { }^{5} \mathrm{P}_{2}-w^{3} \mathrm{~F}_{3} \\
a & { }^{3} \mathrm{~F}_{3}-19_{4}^{\circ} \\
a & { }^{5} \mathrm{~F}_{3}-x{ }^{3} \mathrm{~F}_{3}^{\circ} \\
a & { }^{5} \mathrm{P}_{1}-41_{2}^{\circ}\end{array}$ \\
\hline $\begin{array}{r}1 \\
50 \\
20 \\
1 \\
100\end{array}$ & $\begin{array}{l}2510.504 \\
2510.965 \\
2511.98 \\
2512.47 \\
2512.81\end{array}$ & $\begin{array}{l}39820.65 \\
39813.34 \\
39797.25 \\
39789.49 \\
39784.11\end{array}$ & $\begin{array}{ll}a & { }^{5} \mathbf{F}_{2}-w^{3} \mathbf{D}_{2}^{\circ} \\
b & { }^{3} \mathbf{F}_{4}-43_{3}^{\circ} \\
a & { }^{5} \mathbf{P}_{1}-50_{2}^{\circ} \\
a & { }^{5} \mathbf{F}_{3}-v^{3} \mathbf{D}_{3}^{\circ} \\
a^{3} & { }^{3} \mathbf{F}_{3}-28_{3}^{\circ}\end{array}$ & $\begin{array}{r}1 \\
20 \\
40 \\
50 \\
4\end{array}$ & $\begin{array}{l}2553.288 \\
2553.971 \\
2556.004 \\
2556.316 \\
2556.948\end{array}$ & $\begin{array}{l}39153.44 \\
39142.97 \\
39111.84 \\
39107.07 \\
39097.40\end{array}$ & $\begin{array}{ll}a & 3 \mathrm{G}_{5}-61_{4}^{\circ} \\
a & { }^{3} \mathrm{~F}_{2}-33_{2}^{\circ} \\
a^{5} & \mathrm{D}_{0}-39_{1}^{\circ} \\
a^{5} & \mathrm{D}_{2}-32_{2}^{\circ} \\
a^{5} & \mathrm{P}_{3}-28_{3}^{\circ}\end{array}$ \\
\hline $\begin{array}{r}10 \\
3 \\
30 \\
10 \\
80\end{array}$ & $\begin{array}{l}2513.17 \\
2513.98 \\
2514.45 \\
2514.88 \\
2515.27\end{array}$ & $\begin{array}{l}39778.41 \\
39765.59 \\
39758.16 \\
39751.36 \\
39745.20\end{array}$ & $\begin{array}{ll}a & { }^{5} \mathrm{D}_{4}-19_{4}^{\circ} \\
a & { }^{5} \mathrm{P}_{2}-26_{1}^{\circ} \\
a & { }^{5} \mathrm{~F}_{4}-z^{1} \mathrm{~F}_{3}^{\circ} \\
a & { }^{5} \mathrm{D}_{3}-33_{2}^{\circ} \\
a^{5} \mathrm{P}_{2}-25_{3}^{\circ}\end{array}$ & $\begin{array}{r}10 \\
100 \\
5 \\
125 \\
10\end{array}$ & $\begin{array}{l}2557.701 \\
2558.540 \\
2559.406 \\
2560.265 \\
2561.803\end{array}$ & $\begin{array}{l}39085.89 \\
39073.07 \\
39059.85 \\
39046.75 \\
39023.31\end{array}$ & $\begin{array}{l}a{ }^{5} \mathrm{~F}_{4}-y{ }^{3} \mathrm{G}_{4}^{\circ} \\
a \\
{ }^{3} \mathrm{~F}_{3}-17_{4}^{\circ} \\
a^{5} \mathrm{D}_{3}-23_{3}^{\circ} \\
a{ }^{5} \mathrm{P}_{3}-27_{4}^{\circ} \\
b^{3} \mathrm{~F}_{4}-31_{4}^{\circ}\end{array}$ \\
\hline $\begin{array}{r}20 \\
1 \\
10\end{array}$ & $\begin{array}{l}2515.98 \\
2516.34 \\
2516.73\end{array}$ & $\begin{array}{l}\text { 39733. } 98 \\
\text { 39728. } 30 \\
\text { 39722. } 14\end{array}$ & $\begin{array}{ll}a & { }^{3} \mathbf{F}_{3}-27_{4}^{\circ} \\
a & { }^{3} \mathrm{P}_{2}-59_{3}^{\circ} \\
a^{3} \mathbf{F}_{4}-12_{4}^{\circ} \\
a^{5} & { }^{5} \mathrm{P}_{3}-35_{3}^{\circ}\end{array}$ & $\begin{array}{r}1 \\
3 \\
50 \\
10 \\
10\end{array}$ & $\begin{array}{l}2562.168 \\
2562.55 \\
2563.157 \\
2564.39 \\
2564.41\end{array}$ & $\begin{array}{l}39017.75 \\
39011.93 \\
39002.70 \\
38983.95 \\
38983.64\end{array}$ & $\begin{array}{l}a{ }^{5} \mathrm{P}_{3}-25_{3}^{\circ} \\
a^{5} \mathrm{D}_{4}-x^{1} \mathrm{H}_{5}^{\circ} \\
a{ }^{5} \mathrm{P}_{2}-16_{3}^{\circ} \\
a{ }^{5} \mathrm{P}_{1}-39_{1}^{\circ} \\
a^{3} \mathrm{~F}_{4}-x^{1} \mathrm{G}_{4}^{\circ}\end{array}$ \\
\hline $\begin{array}{l}80 \\
80 \\
10 \\
30 \\
50\end{array}$ & $\begin{array}{l}2517.62 \\
2521.61 \\
2522.318 \\
2525.17 \\
2525.63\end{array}$ & $\begin{array}{ll}39708 . & 10 \\
39645 . & 28 \\
39634 . & 15 \\
39589 . & 39 \\
39582 . & 18\end{array}$ & $\begin{array}{ll}a & { }^{5} \mathrm{D}_{2}-40_{3}^{\circ} \\
b & { }^{3} \mathrm{~F}_{4}-40_{3}^{\circ} \\
a & { }^{5} \mathrm{P}_{3}-34_{3}^{\circ} \\
a & { }^{5} \mathrm{D}_{3}-32_{2}^{\circ} \\
a^{3} & { }^{3} \mathrm{~F}_{2}-40_{3}^{\circ}\end{array}$ & $\begin{array}{l}20 \\
20 \\
10 \\
30 \\
40\end{array}$ & $\begin{array}{l}2564.587 \\
2565.188 \\
2565.804 \\
2566.590 \\
2567.893\end{array}$ & $\begin{array}{l}38980.95 \\
38971.82 \\
38962.46 \\
38950.53 \\
38930.77\end{array}$ & $\begin{array}{l}a{ }^{3} \mathrm{~F}_{2}-32_{2}^{\circ} \\
a^{5} \mathrm{D}_{3}-s^{3} \mathrm{D}_{2}^{\circ} \\
a^{2} \mathrm{~F}_{3}-16_{3}^{\circ} \\
a^{5} \mathrm{D}_{3}-22_{3}^{\circ} \\
a^{3} \mathrm{~F}_{4}-6_{3}^{\circ}\end{array}$ \\
\hline $\begin{array}{r}3 \\
200 \\
3 \\
10 \\
50\end{array}$ & $\begin{array}{l}2525.92 \\
2526.82 \\
2527.347 \\
2527.60 \\
2528.71\end{array}$ & $\begin{array}{l}39577.64 \\
39563.54 \\
39555.29 \\
39551.33 \\
39533.97\end{array}$ & $\begin{array}{l}a{ }^{5} \mathrm{~F}_{4}-x^{3} \mathrm{D}_{3}^{\circ} \\
a{ }^{5} \mathrm{D}_{4}-16_{3}^{\circ} \\
a^{5} \mathrm{D}_{0}-45_{1}^{\circ} \\
a \quad{ }^{3} \mathrm{~F}_{3}-23_{3}^{\circ} \\
a^{5} \mathrm{D}_{3}-30_{4}^{\circ}\end{array}$ & $\begin{array}{r}75 \\
50 \\
30 \\
100 \\
100\end{array}$ & $\begin{array}{l}2568.772 \\
2569.729 \\
2570.093 \\
2572.282 \\
2572.412\end{array}$ & $\begin{array}{l}38917.45 \\
38902.95 \\
38897.44 \\
38864.35 \\
38862.38\end{array}$ & $\begin{array}{ll}a & { }^{5} \mathrm{D}_{4}-13_{4}^{\circ} \\
a & { }^{5} \mathrm{P}_{2}-w^{3} \mathbf{F}_{3}^{\circ} \\
a & { }^{5} \mathrm{~F}_{5}-z^{3} \mathrm{H}_{6}^{\circ} \\
a & { }^{5} \mathrm{P}_{3}-23_{3}^{\circ} \\
a^{3} \mathbf{F}_{3}-w^{3} \mathbf{F}_{3}^{\circ}\end{array}$ \\
\hline $\begin{array}{r}75 \\
7 \\
75 \\
8 \\
1\end{array}$ & $\begin{array}{l}2528.874 \\
2529.456 \\
2530.64 \\
2532.01 \\
2532.36\end{array}$ & $\begin{array}{l}\text { 39531. } 40 \\
39522.31 \\
39503.82 \\
39482.45 \\
39476.99\end{array}$ & $\begin{array}{ll}a & { }^{5} \mathrm{D}_{1}-39_{1}^{\circ} \\
a & { }^{3} \mathrm{~F}_{4}-10_{4}^{\circ} \\
a & { }^{5} \mathrm{P}_{2}-s^{3} \mathrm{D}_{2}^{\circ} \\
a & { }^{5} \mathrm{P}_{2}-22_{3}^{\circ} \\
b & { }^{3} \mathrm{~F}_{4}-38_{4}^{\circ}\end{array}$ & $\begin{array}{r}30 \\
20 \\
2 \\
100\end{array}$ & $\begin{array}{l}2575.242 \\
2576.954 \\
2577.518 \\
2578.571\end{array}$ & $\begin{array}{l}38819.68 \\
38793.89 \\
38785.40 \\
38769.56\end{array}$ & $\begin{array}{l}a a^{3} \mathrm{~F}_{4}-y^{1} \mathrm{G}_{4}^{\circ} \\
a^{3} \mathrm{P}_{2}-50_{2}^{\circ} \\
a^{3} \mathrm{P}_{2}-49_{1}^{\circ} \\
a^{5} \mathrm{~F}_{2}-w^{3} \mathrm{D}_{3}^{\circ} \\
a^{5} \mathrm{D}_{3}-20_{2}^{\circ}\end{array}$ \\
\hline
\end{tabular}


Tabie 3-Continued

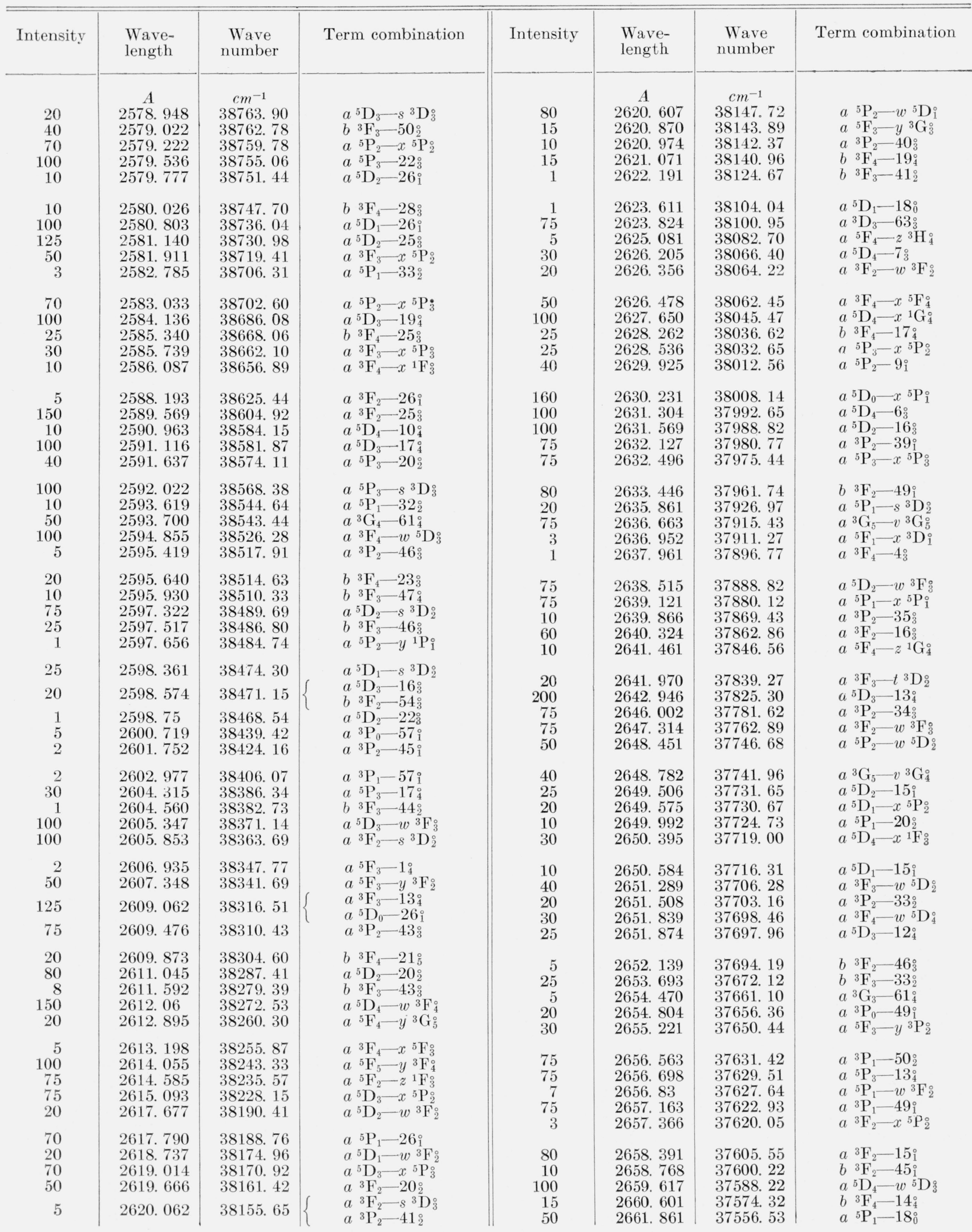


Table 3-Continued

\begin{tabular}{|c|c|c|c|c|c|c|c|}
\hline Intensity & $\begin{array}{l}\text { Wave- } \\
\text { length }\end{array}$ & $\begin{array}{c}\text { Wave } \\
\text { number }\end{array}$ & Term combination & Intensity & $\begin{array}{l}\text { Wave- } \\
\text { length }\end{array}$ & $\begin{array}{c}\text { Wave } \\
\text { number }\end{array}$ & Term combination \\
\hline $\begin{array}{r}100 \\
1 \\
3 \\
25 \\
20\end{array}$ & $\begin{array}{c}A \\
\text { 2664. } 761 \\
\text { 2665. } 154 \\
2665.466 \\
\text { 2665. } 719 \\
2666.829\end{array}$ & $\begin{array}{c}\mathrm{cm}^{-1} \\
37515.66 \\
37510.13 \\
37505.74 \\
37502.18 \\
37486.57\end{array}$ & 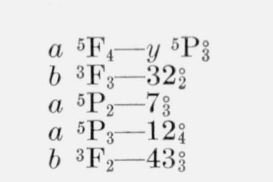 & $\begin{array}{r}125 \\
5 \\
10 \\
2\end{array}$ & $\begin{array}{c}A \\
2709.198 \\
2709.755 \\
2710.732 \\
2711.313 .\end{array}$ & $\begin{array}{c}\mathrm{cm}^{-1} \\
36900.35 \\
36892.77 \\
36879.47 \\
36871.57\end{array}$ & $\begin{array}{ll}a & { }^{5} \mathrm{D}_{3}-6_{3}^{\circ} \\
b & { }^{3} \mathrm{~F}_{3}-s^{3} \mathrm{D}_{2}^{\circ} \\
b & { }^{3} \mathrm{~F}_{2}-33_{2}^{\circ} \\
a & { }^{5} \mathrm{P}_{1}-t^{3} \mathrm{D}_{1}^{\circ} \\
b & { }^{3} \mathrm{~F}_{3}-22_{3}^{\circ}\end{array}$ \\
\hline $\begin{array}{r}10 \\
50 \\
40 \\
2 \\
80\end{array}$ & $\begin{array}{l}2667.484 \\
2667.969 \\
2668.342 \\
2670.708 \\
2673.477\end{array}$ & $\begin{array}{l}37477.36 \\
37470.56 \\
37465.32 \\
37432.13 \\
37393.36\end{array}$ & $\begin{array}{l}a^{3} \mathrm{H}_{5}-62_{\ddagger}^{\circ} \\
a^{5} \mathrm{D}_{2}-y^{1} \mathrm{P}_{1}^{\circ} \\
a^{3} \mathrm{~F}_{3}-7_{3}^{\circ} \\
a^{5} \mathrm{P}_{2}-6_{3}^{\circ} \\
a^{5} \mathrm{D}_{1}-w^{5} \mathrm{D}_{0}^{\circ}\end{array}$ & $\begin{array}{r}10 \\
75 \\
50 \\
1 \\
40\end{array}$ & $\begin{array}{l}2712.868 \\
2713.192 \\
2713.728 \\
2714.998 \\
2715.233\end{array}$ & $\begin{array}{l}36850.44 \\
36846.04 \\
36838.76 \\
36821.53 \\
36818.34\end{array}$ & $\begin{array}{l}a^{5} \mathrm{D}_{1}-t^{3} \mathrm{D}_{2}^{\circ} \\
a^{5} \mathrm{P}_{1}-w^{5} \mathrm{D}_{0}^{\circ} \\
a^{5} \mathrm{D}_{4}-x^{5} \mathrm{~F}_{5}^{\circ} \\
a^{5} \mathrm{P}_{3}-x^{5} \mathrm{~F}_{2}^{\circ} \\
a^{3} \mathrm{P}_{1}-39_{1}^{\circ}\end{array}$ \\
\hline $\begin{array}{r}80 \\
3 \\
3 \\
1 \\
20\end{array}$ & $\begin{array}{l}2673.605 \\
2674.471 \\
2674.827 \\
2676.71 \\
2676.969\end{array}$ & $\begin{array}{l}37391.57 \\
37379.46 \\
37374.49 \\
37348.20 \\
37344.59\end{array}$ & $\begin{array}{ll}a & { }^{3} \mathrm{~F}_{3}-6_{3}^{\circ} \\
a & 3 \mathrm{P}_{2}-29_{3}^{\circ} \\
b & { }^{3} \mathrm{~F}_{4}-x \\
b & { }^{1} \mathrm{H}_{5}^{\circ} \\
b & { }^{3} \mathrm{~F}_{3}-29_{3}^{\circ} \\
a^{3} & { }^{3} \mathrm{~F}_{2}-y^{1} \mathrm{P}_{1}^{\circ}\end{array}$ & $\begin{array}{c}75 \\
30 \\
100 \\
1 h \\
250\end{array}$ & $\begin{array}{l}2715.773 \\
2717.001 \\
2717.401 \\
2718.168 \\
2719.51\end{array}$ & $\begin{array}{l}36811.02 \\
36794.39 \\
36788.97 \\
36778.59 \\
36760.44\end{array}$ & $\begin{array}{l}a{ }^{5} \mathrm{~F}_{1}-y^{3} \mathrm{P}_{1}^{\circ} \\
a^{3} \mathrm{~F}_{2}-8_{1}^{\circ} \\
a^{5} \mathrm{~F}_{1}-y^{3} \mathrm{P}_{0}^{\circ} \\
a{ }^{5} \mathrm{P}_{3}-7_{3}^{\circ} \\
a^{5} \mathrm{D}_{4}-w^{5} \mathrm{D}_{4}^{\circ}\end{array}$ \\
\hline $\begin{array}{r}5 \\
10 \\
50 \\
1 \\
2\end{array}$ & $\begin{array}{l}2677.881 \\
2678.175 \\
2679.763 \\
2680.382 \\
2680.533\end{array}$ & $\begin{array}{l}37331.87 \\
37327.77 \\
37305.65 \\
37297.04 \\
37294.93\end{array}$ & $\begin{array}{l}b{ }^{3} \mathrm{~F}_{2}-41_{2}^{\circ} \\
a{ }^{5} \mathrm{~F}_{1}-y^{3} \mathrm{~F}_{2}^{\circ} \\
a^{3} \mathrm{G}_{4}-v{ }^{3} \mathrm{G}_{5}^{\circ} \\
a^{5} \mathrm{D}_{0}-15_{1}^{\circ} \\
a^{3} \mathrm{P}_{0}-45_{1}^{\circ}\end{array}$ & $\begin{array}{l}70 \\
15 \\
50 \\
10\end{array}$ & $\begin{array}{l}2721.562 \\
2722.386 \\
2722.693 \\
2722.82\end{array}$ & $\begin{array}{l}36732.73 \\
36721.61 \\
36717.47 \\
36715.75\end{array}$ & $\begin{array}{l}a^{5} \mathrm{D}_{2}-w^{5} \mathrm{D}_{2}^{\circ} \\
a^{3} \mathrm{P}_{2}-20_{2}^{\circ} \\
a^{5} \mathrm{D}_{1}-w^{5} \mathrm{D}_{2}^{\circ} \\
b^{3} \mathrm{~F}_{2}-32_{2}^{\circ} \\
a^{3} \mathrm{P}_{2}-s^{3} \mathrm{D}_{3}^{\circ}\end{array}$ \\
\hline $\begin{array}{r}60 \\
60 \\
10 \\
175 \\
40\end{array}$ & $\begin{array}{l}2683.676 \\
2684.089 \\
2684.452 \\
2686.291 \\
2687.138\end{array}$ & $\begin{array}{l}37251.26 \\
37245.53 \\
37240.49 \\
37215.00 \\
37203.27\end{array}$ & $\begin{array}{l}a{ }^{3} \mathrm{P}_{1}-44_{2}^{\circ} \\
a^{1} \mathrm{D}_{2}-63_{3}^{\circ} \\
a^{3} \mathrm{G}_{5}-51_{4}^{\circ} \\
a^{5} \mathrm{D}_{3}-w^{5} \mathrm{D}_{2}^{\circ} \\
a^{5} \mathrm{~F}_{2}-y^{3} \mathrm{P}_{1}^{\circ}\end{array}$ & $\begin{array}{c}100 \\
1 \\
2 \\
50 \\
1 h\end{array}$ & $\begin{array}{l}2724.066 \\
2725.13 \\
2726.360 \\
2726.969 \\
2727.505\end{array}$ & $\begin{array}{l}36698.96 \\
36684.63 \\
36668.08 \\
36659.90 \\
36652.69\end{array}$ & $\begin{array}{l}a{ }^{5} \mathrm{D}_{0}-w^{5} \mathrm{D}_{1}^{\circ} \\
b{ }^{3} \mathrm{~F}_{3}-s^{3} \mathrm{D}_{3}^{\circ} \\
a \\
a^{5} \mathrm{~F}_{1}-y^{5} \mathrm{P}_{1}^{\circ} \\
a^{1} \mathrm{G}_{4}-61_{4}^{\circ} \\
a^{3}{ }^{3} \mathrm{G}_{3}-59_{3}^{\circ}\end{array}$ \\
\hline $\begin{array}{r}1 \\
50 \\
70 \\
70 \\
40\end{array}$ & $\begin{array}{l}2687.755 \\
2688.583 \\
2688.888 \\
2689.894 \\
2690.382\end{array}$ & $\begin{array}{l}37194.73 \\
37183.28 \\
37179.06 \\
37165.15 \\
37158.41\end{array}$ & $\begin{array}{l}a^{3} \mathrm{H}_{4}-63_{3}^{\circ} \\
a \quad \mathrm{P}_{1}-x^{5} \mathrm{P}_{2}^{\circ} \\
a^{5} \mathrm{D}_{4}-y^{3} \mathrm{H}_{4}^{\circ} \\
a^{3} \mathrm{P}_{2}-25_{3}^{\circ} \\
a^{5} \mathrm{P}_{2}-x^{1} \mathrm{~F}_{3}^{\circ}\end{array}$ & $\begin{array}{r}2 \\
80 \\
2 \\
60 \\
1\end{array}$ & $\begin{array}{l}\text { 2727. } 953 \\
2728.836 \\
2729.129 \\
2729.455 \\
2729.790\end{array}$ & $\begin{array}{l}36646.67 \\
36634.81 \\
36630.88 \\
36626.51 \\
36622.01\end{array}$ & $\begin{array}{l}a^{3} \mathrm{G}_{5}-42_{4}^{\circ} \\
b^{3} \mathrm{~F}_{4}-w^{3} \mathrm{~F}_{4}^{\circ} \\
a^{3} \mathrm{G}_{4}-51_{4}^{\circ} \\
a^{5} \mathrm{D}_{3}-x^{1} \mathrm{~F}_{3}^{\circ} \\
a^{5} \mathrm{D}_{3}-y^{5} \mathrm{~S}_{2}^{\circ}\end{array}$ \\
\hline $\begin{array}{r}2 \\
25 \\
50 \\
1\end{array}$ & $\begin{array}{l}2690.725 \\
2690.810 \\
2692.251 \\
2692.716\end{array}$ & $\begin{array}{l}37153.67 \\
37152.50 \\
37132.62 \\
37126.21\end{array}$ & $\begin{array}{l}a{ }^{5} \mathrm{P}_{2}-y{ }^{5} \mathrm{~S}_{2}^{\circ} \\
b^{3} \mathrm{~F}_{4}-12_{4}^{\circ} \\
a^{5} \mathrm{P}_{3}-t^{3} \mathrm{D}_{2}^{\circ} \\
a^{3} \mathrm{G}_{4}-v^{3} \mathrm{G}_{4}^{\circ} \\
b^{3} \mathrm{P}_{2}-60_{1}^{\circ}\end{array}$ & $\begin{array}{r}80 \\
100 \\
30 \\
10 \\
125\end{array}$ & $\begin{array}{l}2730.325 \\
2730.932 \\
2731.903 \\
2733.086 \\
2733.578\end{array}$ & $\begin{array}{l}36614.84 \\
36606.70 \\
36593.69 \\
36577.85 \\
36571.27\end{array}$ & $\begin{array}{l}a{ }^{5} \mathrm{~F}_{3}-y{ }^{5} \mathrm{P}_{3}^{\circ} \\
a^{3} \mathrm{~F}_{2}-u^{5} \mathrm{D}_{2} \\
a^{5} \mathrm{P}_{3}-y{ }^{1} \mathrm{G}_{4}^{\circ} \\
a^{3} \mathrm{~F}_{3}-y^{3} \mathrm{H}_{4}^{\circ} \\
a^{5} \mathrm{P}_{1}-w^{5} \mathrm{D}_{1}^{\circ}\end{array}$ \\
\hline $\begin{array}{r}80 \\
150 \\
30 \\
50 \\
15\end{array}$ & $\begin{array}{l}2692.842 \\
2693.30 \\
2693.653 \\
2697.510 \\
2698.050\end{array}$ & $\begin{array}{l}37124.47 \\
37118.16 \\
37113.29 \\
37060.23 \\
37052.81\end{array}$ & $\begin{array}{l}a{ }^{5} \mathrm{D}_{4}-x^{5} \mathbf{F}_{4} \\
a^{5} \mathrm{D}_{1}-w^{5} \mathrm{D}_{1}^{\circ} \\
a{ }^{3} \mathbf{F}_{3}-y \\
a^{5} \mathbf{S}_{2}^{\circ} \\
a^{5} \mathbf{F}_{2}-y^{5} \mathbf{P}_{1}^{\circ} \\
a^{5} \mathbf{F}_{4}-y^{3} \mathbf{F}_{4}^{\circ}\end{array}$ & $\begin{array}{r}1 \\
250 \\
10 \\
1 \\
20\end{array}$ & $\begin{array}{l}2734.15 \\
2735.727 \\
2736.315 \\
2737.145 \\
2737.463\end{array}$ & $\begin{array}{l}36563.62 \\
36542.54 \\
36534.69 \\
26523.61 \\
36519.37\end{array}$ & $\begin{array}{l}a{ }^{5} \mathrm{D}_{0}-9_{1}^{\circ} \\
a{ }^{5} \mathrm{~F}_{5}-x^{5} \mathrm{D}_{4}^{\circ} \\
a^{5} \mathrm{D}_{2}-x{ }^{5} \mathrm{~F}_{2}^{\circ} \\
a^{3} \mathrm{~F}_{3}-x^{5} \mathrm{~F}_{4}^{\circ} \\
a^{5} \mathrm{D}_{1}-x^{5} \mathrm{~F}_{2}^{\circ}\end{array}$ \\
\hline $\begin{array}{l}10 \\
20 \\
40 \\
30 \\
25\end{array}$ & $\begin{array}{l}\text { 2699. } 712 \\
2699.793 \\
2699.882 \\
2700.477 \\
2700.671\end{array}$ & $\begin{array}{l}37030.00 \\
37028.89 \\
37027.67 \\
37019.51 \\
37016.85\end{array}$ & $\begin{array}{l}a^{5} \mathrm{D}_{1}-11_{0}^{\circ} \\
a{ }^{5} \mathbf{F}_{2}-y^{3} \mathrm{P}_{2}^{\circ} \\
a \quad \mathrm{P}_{2}-w^{5} \mathrm{D}_{3}^{\circ} \\
a^{5} \mathrm{P}_{3}-w^{5} \mathrm{D}_{2}^{\circ} \\
a^{5} \mathrm{D}_{3}-x^{5} \mathrm{~F}_{2}^{\circ}\end{array}$ & $\begin{array}{c}150 \\
5 \\
1 h \\
50 \\
80\end{array}$ & $\begin{array}{l}2739.217 \\
2739.454 \\
2739.986 \\
2740.217 \\
2743.934\end{array}$ & $\begin{array}{l}36495.98 \\
36492.83 \\
36485.74 \\
36482.67 \\
36433.25\end{array}$ & $\begin{array}{l}a^{5} \mathrm{D}_{3}-w^{5} \mathrm{D}_{3}^{\circ} \\
a{ }^{5} \mathrm{D}_{4}-x^{3} \mathrm{G}_{3}^{\circ} \\
a^{5} \mathrm{D}_{0}-8_{1}^{\circ} \\
a{ }^{5} \mathrm{P}_{1}-11_{0}^{\circ} \\
a^{3} \mathrm{~F}_{4}-z^{3} \mathrm{I}_{5}^{\circ}\end{array}$ \\
\hline $\begin{array}{r}70 \\
5 \\
4 \\
80 \\
4\end{array}$ & $\begin{array}{l}2701.337 \\
2701.997 \\
2702.401 \\
2702.833 \\
2703.120\end{array}$ & $\begin{array}{l}37007.73 \\
36998.69 \\
36993.16 \\
36987.25 \\
36983.32\end{array}$ & $\begin{array}{l}a{ }^{3} \mathrm{~F}_{2}-w^{5} \mathrm{D}_{1} \\
a^{5} \mathrm{D}_{2}-9_{1} \\
a^{3} \mathrm{P}_{1}-41_{2}^{\circ} \\
a^{3} \mathrm{~F}_{3}-w^{5} \mathrm{D}_{3}^{\circ} \\
a^{5} \mathrm{D}_{1}-9_{1}^{\circ}\end{array}$ & $\begin{array}{c}125 \\
3 h \\
75 \\
15 \\
75\end{array}$ & $\begin{array}{l}2744.448 \\
2744.705 \\
2745.074 \\
2745.827 \\
2746.885\end{array}$ & $\begin{array}{l}36426.43 \\
36423.01 \\
36418.12 \\
36408.13 \\
36394.11\end{array}$ & $\begin{array}{ll}a & { }^{5} \mathrm{P}_{3}-y{ }^{5} \mathrm{~S}_{2}^{\circ} \\
a & { }^{3} \mathrm{P}_{2}-16_{3}^{\circ} \\
a^{5} \mathrm{D}_{2}-66_{3}^{\circ} \\
b{ }^{3} \mathrm{~F}_{4}-x^{1} \mathrm{G}_{4}^{\circ} \\
a^{3} \mathrm{~F}_{4}-x^{3} \mathrm{G}_{4}^{\circ}\end{array}$ \\
\hline $\begin{array}{r}4 \\
50 \\
5 \\
10 \\
70\end{array}$ & $\begin{array}{l}2703.311 \\
2703.796 \\
2704.971 \\
2705.323 \\
2707.477\end{array}$ & $\begin{array}{l}\text { 36980. } 71 \\
36974.07 \\
\text { 36958. } 01 \\
\text { 36953. } 20 \\
\text { 36923. } 81\end{array}$ & $\begin{array}{l}b{ }^{3} \mathrm{~F}_{3}-23_{3}^{\circ} \\
a^{5} \mathrm{D}_{3}-7_{3}^{\circ} \\
a^{3} \mathrm{G}_{5}-47_{4}^{\circ} \\
b^{3} \mathrm{~F}_{2}-34_{3}^{\circ} \\
a^{5} \mathrm{D}_{3}-x^{1} \mathrm{G}_{4}^{\circ} \\
a^{3} \mathrm{P}_{2}-s^{3} \mathrm{D}_{2}^{\circ}\end{array}$ & $\begin{array}{r}1 \\
40 \\
40 \\
10 \\
2\end{array}$ & $\begin{array}{l}2747.163 \\
2747.963 \\
2748.045 \\
2749.014 \\
2749.314\end{array}$ & $\begin{array}{l}36390.43 \\
36379.83 \\
36378.75 \\
36365.93 \\
36361.96\end{array}$ & $\begin{array}{l}a^{3} \mathrm{G}_{5}-38_{4}^{\circ} \\
a^{5} \mathrm{D}_{4}-w^{3} \mathrm{G}_{4}^{\circ} \\
a^{3} \mathrm{P}_{1}-32_{2}^{\circ} \\
a^{3} \mathrm{~F}_{2}-7_{3}^{\circ} \\
b^{3} \mathrm{~F}_{2}-26_{1}^{\circ}\end{array}$ \\
\hline $\begin{array}{r}100 \\
25 \\
25 \\
5\end{array}$ & $\begin{array}{l}2707.969 \\
2708.635 \\
2708.841 \\
2709.045\end{array}$ & $\begin{array}{l}36917.10 \\
36908.02 \\
36905.21 \\
36902.44\end{array}$ & $\begin{array}{l}a{ }^{5} \mathrm{~F}_{3}-y{ }^{5} \mathrm{P}_{2} \\
a{ }^{5} \mathrm{P}_{1}-y \\
a^{5} \mathrm{P}_{1} \\
{ }^{5} \mathrm{D}_{1}-8_{1}^{\circ} \\
a^{3} \mathrm{G}_{4}-52_{4}^{\circ} \\
a^{3} \mathrm{P}_{2}-22_{3}^{\circ}\end{array}$ & $\begin{array}{c}5 h \\
3 \\
50 \\
2\end{array}$ & $\begin{array}{l}2749.64 \\
2749.824 \\
2750.345 \\
2750.864\end{array}$ & $\begin{array}{l}36357.65 \\
36355.21 \\
36348.33 \\
36341.47\end{array}$ & $\begin{array}{l}a{ }^{3} \mathrm{~F}_{3}-4_{3}^{\circ} \\
a^{5} \mathrm{P}_{1}-8_{1}^{\circ} \\
b^{3} \mathrm{~F}_{4}-6{ }_{3}^{\circ} \\
a^{3} \mathrm{G}_{4}-47_{4}^{\circ} \\
b^{3} \mathrm{~F}_{2}-25_{3}^{\circ}\end{array}$ \\
\hline
\end{tabular}


TABLE 3-Continued

\begin{tabular}{|c|c|c|c|c|c|c|c|}
\hline Intensity & $\begin{array}{l}\text { Wave- } \\
\text { length }\end{array}$ & $\begin{array}{c}\text { Wave } \\
\text { number }\end{array}$ & Term combination & Intensity & $\begin{array}{l}\text { Wave- } \\
\text { length }\end{array}$ & $\begin{array}{c}\text { Wave } \\
\text { number }\end{array}$ & Term combination \\
\hline $\begin{array}{r}60 \\
1 \\
10 \\
10 \\
60\end{array}$ & $\begin{array}{c}A \\
2752.262 \\
2753.761 \\
2753.968 \\
2754.358 \\
2754.603\end{array}$ & $\begin{array}{c}\mathrm{cm}^{-1} \\
36323.01 \\
36303.24 \\
36300.51 \\
36295.37 \\
36292.14\end{array}$ & $\begin{array}{l}a{ }^{3} \mathrm{P}_{2}-w^{3} \mathrm{~F}_{3}^{\circ} \\
a^{5} \mathrm{P}_{1}-t^{3} \mathrm{D}_{2}^{\circ} \\
a^{5} \mathrm{P}_{3}-w^{5} \mathrm{D}_{3}^{\circ} \\
a^{5} \mathbf{F}_{2}-y^{5} \mathrm{P}_{2}^{\circ} \\
a^{3} \mathbf{F}_{2}-6_{3}^{\circ}\end{array}$ & $\begin{array}{r}50 \\
150 \\
250 \\
20 \\
25\end{array}$ & $\begin{array}{c}A \\
2808.221 \\
2810.029 \\
2810.551 \\
2810.695 \\
2814.862\end{array}$ & $\begin{array}{c}\mathrm{cm}^{-1} \\
\text { 35599. } 25 \\
\text { 35576. } 34 \\
\text { 35569. } 74 \\
35567.92 \\
\text { 35515. } 26\end{array}$ & $\begin{array}{ll}b & { }^{3} \mathrm{~F}_{2}-16_{3}^{\circ} \\
a & 5 \mathrm{~F}_{3}-x^{5} \mathrm{D}_{2}^{\circ} \\
a^{5} \mathrm{~F}_{4}-y^{3} \mathrm{D}_{3}^{\circ} \\
a^{3} \mathrm{P}_{2}-w^{5} \mathrm{D}_{1}^{\circ} \\
a^{5} \mathrm{D}_{4}-y^{1} \mathrm{~F}_{3}^{\circ}\end{array}$ \\
\hline $\begin{array}{r}1 \\
30 \\
80 \\
2 \\
100\end{array}$ & $\begin{array}{l}2756.181 \\
2757.064 \\
2757.798 \\
2758.238 \\
2759.682\end{array}$ & $\begin{array}{l}36271.37 \\
36259.75 \\
36250.10 \\
36244.32 \\
36225.35\end{array}$ & $\begin{array}{l}a{ }^{3} \mathrm{G}_{3}-55_{2}^{\circ} \\
a^{5} \mathrm{D}_{4}-w^{3} \mathrm{G}_{5}^{\circ} \\
a^{3} \mathrm{G}_{3}-v^{3} \mathrm{G}_{4}^{\circ} \\
b^{3} \mathbf{F}_{4}-y{ }^{1} \mathbf{G}_{4} \\
a^{5} \mathrm{D}_{3}-x{ }^{5} \mathbf{F}_{3}^{\circ}\end{array}$ & $\begin{array}{r}1 \\
100 \\
200 \\
40 \\
75\end{array}$ & $\begin{array}{l}2816.116 \\
2817.092 \\
2818.359 \\
2818.809 \\
2818.950\end{array}$ & $\begin{array}{l}35499.45 \\
35487.15 \\
35471.20 \\
35465.53 \\
35463.76\end{array}$ & $\begin{array}{ll}b & { }^{3} \mathrm{~F}_{2}-w^{3} \mathrm{~F}_{3}^{\circ} \\
a & { }^{5} \mathrm{~F}_{2}-x^{5} \mathrm{D}_{1} \\
a & { }^{5} \mathrm{~F}_{5}-y^{5} \mathrm{~F}_{4}^{\circ} \\
a & { }^{5} \mathrm{P}_{2}-u^{3} \mathrm{D}_{2}^{\circ} \\
a^{5} \mathrm{P}_{3}-x^{3} \mathrm{P}_{2}^{\circ}\end{array}$ \\
\hline $\begin{array}{r}30 \\
100 \\
60 \\
200 \\
50\end{array}$ & $\begin{array}{l}2760.155 \\
2762.304 \\
2763.133 \\
2763.413 \\
2763.900\end{array}$ & $\begin{array}{l}36219.15 \\
36190.97 \\
36180.12 \\
36176.45 \\
36170.07\end{array}$ & $\begin{array}{l}a{ }^{3} \mathrm{G}_{3}-54_{3}^{\circ} \\
a^{5} \mathrm{P}_{2}-x^{3} \mathrm{P}_{2}^{\circ} \\
a^{3} \mathrm{P}_{2}-x{ }^{5} \mathrm{P}_{2}^{0} \\
a^{5} \mathrm{~F}_{4}-x^{5} \mathrm{D}_{3}^{\circ} \\
a^{5} \mathrm{P}_{1}-w^{5} \mathrm{D}_{2}^{\circ}\end{array}$ & $\begin{array}{r}5 \\
1 \\
30 \\
3 \\
100\end{array}$ & $\begin{array}{l}2819.562 \\
2820.667 \\
2821.171 \\
2821.414 \\
2822.034\end{array}$ & $\begin{array}{l}35456.06 \\
35442.17 \\
35435.84 \\
35432.79 \\
35425.01\end{array}$ & $\begin{array}{l}a{ }^{5} \mathrm{D}_{4}-x^{3} \mathrm{G}_{4}^{\circ} \\
a^{3} \mathrm{G}_{3}-46_{3}^{\circ} \\
b{ }^{3} \mathrm{P}_{1}-50_{2}^{\circ} \\
a^{3} \mathrm{P}_{2}-9_{1}^{\circ} \\
a^{3}{ }^{3} \mathrm{~F}_{3}-u^{3} \mathrm{D}_{2}^{\circ}\end{array}$ \\
\hline $\begin{array}{r}10 \\
70 \\
5 \\
1 \\
50\end{array}$ & $\begin{array}{l}\text { 2764. } 229 \\
2764.714 \\
2765.521 \\
2765.853 \\
2766.223\end{array}$ & $\begin{array}{l}36165.77 \\
36159.43 \\
36148.87 \\
36144.54 \\
36139.70\end{array}$ & $\begin{array}{l}a^{3} \mathrm{P}_{2}-15_{1}^{\circ} \\
a^{3} \mathbf{F}_{3}-w^{5} \mathrm{D}_{4}^{\circ} \\
b^{3} \mathbf{F}_{3}-x^{5} \mathrm{P}_{2}^{\circ} \\
a^{5} \mathrm{D}_{2}-x^{1} \mathbf{F}_{3}^{\circ} \\
a^{5} \mathrm{D}_{2}-y^{5} \mathbf{S}_{2}^{\circ}\end{array}$ & $\begin{array}{r}1 \\
2 \\
10 \\
5 \\
100\end{array}$ & $\begin{array}{l}2822.256 \\
2823.878 \\
2824.756 \\
2827.518 \\
2827.857\end{array}$ & $\begin{array}{l}35422.22 \\
35401.88 \\
35390.87 \\
35356.30 \\
35352.07\end{array}$ & $\begin{array}{ll}a & { }^{1} \mathrm{G}_{4}-v^{3} \mathrm{G}_{5}^{\circ} \\
b & { }^{3} \mathrm{P}_{2}-45_{1}^{\circ} \\
a^{3} & \mathrm{P}_{1}-18_{0}^{\circ} \\
b & { }^{3} \mathrm{~F}_{2}-x^{5} \mathrm{P}_{2}^{\circ} \\
a^{5} \mathrm{~F}_{4}-x{ }^{5} \mathrm{D}_{4}^{\circ}\end{array}$ \\
\hline $\begin{array}{c}1 \\
50 w \\
1 \\
1 \\
1 h\end{array}$ & $\begin{array}{l}2767.389 \\
2767.516 \\
2767.689 \\
2767.938 \\
2769.893\end{array}$ & $\begin{array}{l}36124.48 \\
36122.82 \\
36120.56 \\
36117.31 \\
36091.82\end{array}$ & $\begin{array}{ll}a^{5} \mathrm{D}_{1}-y^{5} \mathrm{~S}_{2}^{\circ} \\
a & { }^{3} \mathrm{P}_{2}-x^{5} \mathrm{P}_{3}^{\circ} \\
a & { }^{5} \mathrm{P}_{3}-5_{2}^{\circ} \\
a & { }^{3} \mathrm{G}_{4}-43_{3}^{\circ} \\
b & { }^{3} \mathrm{~F}_{3}-x^{5} \mathrm{P}_{3}^{\circ}\end{array}$ & $\begin{array}{r}200 \\
1 \\
40 \\
20 \\
80\end{array}$ & $\begin{array}{ll}2829.149 \\
2832.044 \\
2832.624 \\
2833.05 \\
2833.999\end{array}$ & $\begin{array}{l}35335.92 \\
35299.80 \\
35292.57 \\
35287.27 \\
35275.45\end{array}$ & $\begin{array}{l}a{ }^{3} \mathrm{~F}_{4}-v^{3} \mathrm{D}_{3}^{\circ} \\
a{ }^{3} \mathrm{P}_{2}-t^{3} \mathrm{D}_{2}^{\circ} \\
a^{3} \mathrm{G}_{4}-30_{4}^{\circ} \\
a^{5} \mathrm{D}_{3}-w^{3} \mathrm{G}_{4}^{\circ} \\
a^{5} \mathrm{~F}_{3}-x^{5} \mathrm{D}_{3}^{\circ}\end{array}$ \\
\hline $\begin{array}{c}50 \\
100 \\
100 \\
1 h \\
125\end{array}$ & $\begin{array}{l}2770.296 \\
2770.698 \\
2772.608 \\
2774.101 \\
2774.480\end{array}$ & $\begin{array}{l}36086.57 \\
36081.33 \\
36056.48 \\
36037.08 \\
36032.15\end{array}$ & $\begin{array}{l}a{ }^{5} \mathrm{D}_{3}-y{ }^{3} \mathrm{H}_{4}^{\circ} \\
b{ }^{3} \mathbf{F}_{4}-x{ }^{1} \mathbf{F}_{3}^{\circ} \\
a{ }^{3} \mathbf{P}_{0}-26_{1}^{\circ} \\
a^{3} \mathrm{G}_{4}-42_{4}^{\circ} \\
a^{5} \mathrm{D}_{3}-x^{5} \mathbf{F}_{4}^{\circ}\end{array}$ & $\begin{array}{r}2 \\
2 \\
50 \\
70 \\
20\end{array}$ & $\begin{array}{l}\text { 2834. } 347 \\
2835.415 \\
2836.143 \\
2836.569 \\
2837.269\end{array}$ & $\begin{array}{l}35271.12 \\
35257.84 \\
35248.79 \\
35243.49 \\
35234.80\end{array}$ & $\begin{array}{l}a \\
{ }^{5} \mathrm{P}_{1}-5_{2}^{\circ} \\
a^{3} \mathrm{~F}_{2}-4_{3}^{\circ} \\
a^{1} \mathrm{G}_{4}-v^{3} \mathrm{G}_{4}^{\circ} \\
a^{5} \mathrm{D}_{2}-3_{1}^{\circ} \\
a^{3} \mathrm{G}_{3}-43_{3}^{\circ}\end{array}$ \\
\hline 70 & 2775.175 & 36023. 13 & $a^{3} \mathrm{G}_{3}-52_{4}^{\circ}$ & 3 & 2837.814 & 35228. 03 & $a{ }^{5} \mathrm{D}_{1}-3{ }_{1}^{\circ}$ \\
\hline $\begin{array}{c}2 h \\
80\end{array}$ & $\begin{array}{l}2775.540 \\
2775.902\end{array}$ & $\begin{array}{l}\text { 36018. } 39 \\
\text { 36013. } 70 \\
36013.71\end{array}$ & $\begin{array}{l}a a^{3} \mathbf{F}_{2}-x^{1} \mathbf{F}_{3}^{\circ} \\
a^{5} \mathrm{D}_{2}-w^{5} \mathrm{D}_{3}^{\circ} \\
a^{3} \mathbf{F}_{2}-y^{5} \mathbf{S}_{2}^{\circ}\end{array}$ & $\begin{array}{r}25 \\
1 \\
80\end{array}$ & $\begin{array}{l}2838.615 \\
2839.677 \\
2840.537\end{array}$ & $\begin{array}{l}35218.09 \\
35204.92 \\
35194.26\end{array}$ & $\begin{array}{l}a^{3} \mathrm{G}_{5}-21_{5}^{\circ} \\
a{ }^{1} \mathrm{G}_{4}-54_{3}^{\circ} \\
a \\
a^{5} \mathrm{P}_{3}-x^{3} \mathrm{G}_{3}^{\circ} \\
a^{3}{ }^{3} \mathrm{~F}_{4}-x^{3} \mathrm{G}_{5}^{\circ}\end{array}$ \\
\hline $\begin{array}{r}5 \\
70 \\
5 \\
80 \\
3\end{array}$ & $\begin{array}{l}2777.480 \\
2780.759 \\
2781.831 \\
2782.205 \\
2784.320\end{array}$ & $\begin{array}{l}35993.24 \\
35950.80 \\
35936.94 \\
35932.11 \\
35904.82\end{array}$ & $\begin{array}{ll}a & { }^{5} \mathbf{F}_{2}-y{ }^{5} \mathbf{P}_{3}^{\circ} \\
b & { }^{3} \mathbf{F}_{4}-w^{5} \mathbf{D}_{3}^{\circ} \\
a^{3} \mathbf{G}_{5}-31_{4}^{\circ} \\
a^{5}{ }^{5} \mathbf{P}_{2}-x^{3} \mathbf{G}_{3}^{\circ} \\
a^{3} \mathbf{P}_{2}-y^{1} \mathbf{P}_{1}^{\circ}\end{array}$ & $\begin{array}{l}2 \\
2 h \\
20 \\
20 \\
70\end{array}$ & $\begin{array}{l}2841.173 \\
2841.928 \\
2842.527 \\
2842.749 \\
2843.170\end{array}$ & $\begin{array}{l}35186.39 \\
35177.04 \\
35169.63 \\
35166.88 \\
35161.67\end{array}$ & $\begin{array}{l}a^{3} \mathrm{G}_{4}-29_{3}^{\circ} \\
a^{5} \mathrm{D}_{2}-x^{3} \mathrm{P}_{2}^{\circ} \\
a^{3} \mathrm{D}_{3}-61_{4}^{\circ} \\
a^{3} \mathrm{P}_{2}-w^{5} \mathrm{D}_{2}^{\circ} \\
a^{5} \mathrm{D}_{1}-x^{3} \mathrm{P}_{2}^{\circ}\end{array}$ \\
\hline $\begin{array}{r}30 \\
2 \\
75 \\
80 \\
5\end{array}$ & $\begin{array}{l}2784.516 \\
2784.864 \\
2785.334 \\
2785.649 \\
2786.710\end{array}$ & $\begin{array}{l}35902.29 \\
35897.81 \\
35891.75 \\
35887.69 \\
35874.03\end{array}$ & $\begin{array}{ll}a^{3} \mathrm{G}_{5}-30_{4}^{\circ} \\
b \quad \mathbf{F}_{2}-20_{2}^{\circ} \\
a^{3} \mathbf{F}_{3}-x^{3} \mathbf{G}_{3}^{\circ} \\
a & { }^{3} \mathbf{F}_{2}-w^{5} \mathbf{D}_{3}^{\circ} \\
a & { }^{5} \mathbf{F}_{2}-z^{3} \mathbf{S}_{1}^{\circ}\end{array}$ & $\begin{array}{r}5 \\
25 \\
60 \\
20\end{array}$ & $\begin{array}{l}2843.740 \\
2845.537 \\
2846.318 \\
2846.537\end{array}$ & $\begin{array}{l}35154.62 \\
35132.43 \\
35122.79 \\
35120.08\end{array}$ & $\begin{array}{l}a{ }^{3} \mathrm{G}_{3}-42_{4}^{\circ} \\
a{ }^{5} \mathrm{P}_{3}-y^{1} \mathrm{D}_{2}^{\circ} \\
b{ }^{3} \mathrm{~F}_{4}-w^{5} \mathrm{D}_{4}^{\circ} \\
a^{3}{ }^{3} \mathrm{H}_{5}-61_{4}^{\circ} \\
b^{3} \mathrm{P}_{2}-40_{3}^{\circ}\end{array}$ \\
\hline $\begin{array}{r}100 \\
2 \\
1 \\
6 \\
75\end{array}$ & $\begin{array}{l}2787.823 \\
2789.615 \\
2789.833 \\
2791.036 \\
2792.641\end{array}$ & $\begin{array}{l}35859.71 \\
35836.67 \\
35833.87 \\
35818.43 \\
35797.84\end{array}$ & $\begin{array}{l}a{ }^{5} \mathrm{P}_{2}-y{ }^{1} \mathrm{D}_{2}^{\circ} \\
a{ }^{5} \mathrm{P}_{3}-x{ }^{5} \mathrm{~F}_{4}^{\circ} \\
a^{5} \mathrm{D}_{2}-5_{2}^{\circ} \\
a^{5} \mathrm{D}_{1}-5_{2}^{\circ} \\
a^{5} \mathrm{P}_{2}-u^{3} \mathrm{D}_{1}^{\circ}\end{array}$ & $\begin{array}{r}30 \\
80 \\
3 \\
1 \\
2\end{array}$ & $\begin{array}{l}2846.750 \\
2848.586 \\
2848.812 \\
2849.775 \\
2850.864\end{array}$ & $\begin{array}{l}35117.46 \\
35094.82 \\
35092.04 \\
35080.18 \\
35066.78\end{array}$ & $\begin{array}{l}a{ }^{3} \mathrm{~F}_{2}-3{ }_{1}^{\circ} \\
a \\
{ }^{5} \mathrm{~F}_{1}-x^{5} \mathrm{D}_{1}^{\circ} \\
a \\
{ }^{5} \mathrm{P}_{3}-w^{3} \mathrm{G}_{4} \\
a^{3} \mathrm{G}_{3}-41_{2}^{\circ} \\
a^{3} \mathrm{G}_{3}-40_{3}^{\circ}\end{array}$ \\
\hline $\begin{array}{r}20 \\
30 \\
1 \\
20 \\
40\end{array}$ & $\begin{array}{l}2794.678 \\
2795.508 \\
2796.049 \\
2796.543 \\
2796.697\end{array}$ & $\begin{array}{l}35771.75 \\
35761.13 \\
35754.21 \\
35747.90 \\
35745.93\end{array}$ & $\begin{array}{ll}b & { }^{3} \mathrm{P}_{2}-50_{2}^{\circ} \\
a & { }_{3} \mathrm{P}_{1}-s^{3} \mathrm{D}_{2}^{\circ} \\
a & { }^{3} \mathrm{G}_{4}-37_{3}^{\circ} \\
a & { }^{3} \mathrm{P}_{0}-x^{5} \mathrm{P}_{1} \\
b & { }^{3} \mathbf{F}^{3}-13_{4}^{\circ}\end{array}$ & $\begin{array}{r}15 \\
10 \\
200 \\
1 \\
3\end{array}$ & $\begin{array}{l}2851.104 \\
2851.866 \\
2854.075 \\
2854.528 \\
2854.870\end{array}$ & $\begin{array}{l}35063.83 \\
35054.46 \\
35027.33 \\
35021.77 \\
35017.58\end{array}$ & $\begin{array}{l}a{ }^{5} \mathrm{P}_{2}-y^{3} \mathrm{~S}_{1} \\
a^{3} \mathrm{G}_{5}-19_{4}^{\circ} \\
a \\
a^{5} \mathrm{~F}_{3}-z{ }^{3} \mathrm{P}_{2}^{\circ} \\
a^{1}{ }^{1} \mathrm{G}_{4}-52_{4}^{\circ} \\
a^{3} \mathrm{P}_{1}-x{ }^{5} \mathrm{P}_{2}^{\circ}\end{array}$ \\
\hline $\begin{array}{r}1 \\
1 \\
10 \\
125 \\
40\end{array}$ & $\begin{array}{l}\text { 2796. } 904 \\
2798.872 \\
2801.856 \\
2802.805 \\
2803.496\end{array}$ & $\begin{array}{l}\text { 35743. } 28 \\
35718.15 \\
35680.11 \\
35668.03 \\
35659.24\end{array}$ & $\begin{array}{l}a^{5} \mathrm{D}_{2}-x^{5} \mathrm{~F}_{3}^{\circ} \\
a^{3} \mathrm{D}_{2}-60_{1}^{\circ} \\
b^{3} \mathbf{F}_{4}-x^{5} \mathbf{F}_{3}^{\circ} \\
a^{5} \mathrm{D}_{3}-w^{5} \mathrm{D}_{4}^{\circ} \\
a^{5} \mathrm{D}_{3}-x^{3} \mathbf{P}_{2}^{\circ}\end{array}$ & $\begin{array}{r}30 \\
1 \\
2 \\
1 \\
100\end{array}$ & $\begin{array}{l}\text { 2856. } 044 \\
2857.240 \\
2858.591 \\
2858.849 \\
2860.014\end{array}$ & $\begin{array}{l}35003.18 \\
34988.53 \\
34972.00 \\
34968.84 \\
34954.60\end{array}$ & $\begin{array}{ll}a & { }^{3} \mathrm{P}_{1}-15_{1}^{\circ} \\
b & { }^{3} \mathrm{~F}_{4}-y^{3} \mathrm{H}_{5}^{\circ} \\
a^{3} & \mathrm{G}_{4}-25_{3}^{\circ} \\
a^{3} \mathrm{P}_{2}-x^{5} \mathrm{~F}_{2}^{\circ} \\
a^{5} \mathrm{~F}_{2}-x^{5} \mathrm{D}_{2}^{\circ} \\
a^{5}{ }^{5} \mathrm{P}_{2}-y^{1} \mathrm{~F}_{3}^{\circ}\end{array}$ \\
\hline
\end{tabular}


TABLE 3-Continued

\begin{tabular}{|c|c|c|c|c|c|c|c|}
\hline Intensity & $\begin{array}{l}\text { Wave- } \\
\text { length }\end{array}$ & $\begin{array}{c}\text { Wave } \\
\text { number }\end{array}$ & Term combination & Intensity & $\begin{array}{l}\text { Wave- } \\
\text { length }\end{array}$ & $\begin{array}{c}\text { Wave } \\
\text { number }\end{array}$ & Term combination \\
\hline $\begin{array}{r}20 \\
125 \\
40 \\
1\end{array}$ & $\begin{array}{c}\text { A } \\
2860.369 \\
2861.408 \\
2861.718 \\
2862.358\end{array}$ & $\begin{array}{c}c m^{-1} \\
34950.26 \\
34937.57 \\
34933.78 \\
34925.97\end{array}$ & $\begin{array}{l}a{ }^{3} \mathrm{G}_{5}-17_{4}^{\circ} \\
a^{3} \mathrm{~F}_{4}-w^{3} \mathrm{D}_{3}^{\circ} \\
b^{3} \mathrm{~F}_{3}-x^{5} \mathrm{~F}_{2}^{\circ} \\
a^{5} \mathrm{D}_{3}-u^{3} \mathrm{D}_{2}^{\circ} \\
a^{3} \mathrm{P}_{2}-7_{3}^{\circ}\end{array}$ & $\begin{array}{r}75 \\
50 \\
60 \\
2 \\
150\end{array}$ & $\begin{array}{c}\text { A } \\
2905.651 \\
2905.822 \\
2906.315 \\
2908.481 \\
2908.883\end{array}$ & $\begin{array}{c}\mathrm{cm}^{-1} \\
34405.61 \\
34403.59 \\
34397.76 \\
34372.14 \\
34367.39\end{array}$ & $\begin{array}{ll}a & { }^{5} \mathrm{~F}_{2}-z^{3} \mathrm{P}_{2}^{\circ} \\
a & { }^{3} \mathrm{~F}_{4}-z^{1} \mathrm{~F}_{3}^{\circ} \\
a & { }^{5} \mathrm{D}_{4}-v^{3} \mathrm{D}_{3}^{\circ} \\
a & { }^{5} \mathrm{P}_{2}-z^{1} \mathrm{P}_{1}^{\circ} \\
a^{5} \mathrm{~F}_{1}-z^{3} \mathrm{P}_{0}^{\circ}\end{array}$ \\
\hline $\begin{array}{r}30 \\
20 \\
10 \\
1 \\
150\end{array}$ & $\begin{array}{l}2863.003 \\
2863.324 \\
2863.975 \\
2864.618 \\
2866.653\end{array}$ & $\begin{array}{l}34918.11 \\
34914.19 \\
34906.26 \\
34898.42 \\
34873.65\end{array}$ & $\begin{array}{l}a{ }^{5} \mathrm{D}_{2}-x^{3} \mathrm{G}_{3}^{\circ} \\
a^{3} \mathrm{~F}_{3}-y{ }^{1} \mathrm{~F}_{3}^{5} \\
a^{5} \mathrm{~F}_{2}-y{ }^{3} \mathrm{D}_{1}^{\circ} \\
a^{3} \mathrm{G}_{3}-38_{4}^{\circ} \\
a^{5} \mathrm{~F}_{3}-y^{3} \mathrm{D}_{2}^{\circ}\end{array}$ & $\begin{array}{r}30 \\
5 \\
20 \\
10\end{array}$ & $\begin{array}{l}2909.212 \\
2909.940 \\
2910.425 \\
2910.772\end{array}$ & $\begin{array}{l}34363.50 \\
34354.91 \\
34349.18 \\
34345.09\end{array}$ & $\begin{array}{l}a{ }^{5} \mathrm{D}_{3}-x^{3} \mathrm{G}_{4}^{\circ} \\
a{ }^{3} \mathrm{D}_{2}-50_{2}^{\circ} \\
a^{3} \mathrm{D}_{2}-49_{1}^{\circ} \\
a^{5} \mathrm{D}_{0}-u^{3} \mathrm{D}_{\mathrm{i}} \\
b^{3} \mathrm{P}_{1}-33_{2}^{\circ}\end{array}$ \\
\hline $\begin{array}{r}25 \\
25 \\
30 \\
8 \\
15\end{array}$ & $\begin{array}{l}2867.465 \\
2868.183 \\
2868.310 \\
2868.412 \\
2868.544\end{array}$ & $\begin{array}{l}34863.77 \\
34855.05 \\
34853.50 \\
34852.26 \\
34850.66\end{array}$ & $\begin{array}{ll}a & { }^{5} \mathrm{D}_{4}-x^{3} \mathrm{~F}_{4}^{\circ} \\
a & { }^{3} \mathrm{~F}_{3}-x^{3} \mathrm{G}_{4} \\
a & { }^{5} \mathrm{P}_{2}-v^{3} \mathrm{D}_{2} \\
a & { }^{3} \mathrm{P}_{2}-6_{3}^{\circ} \\
a^{5} \mathrm{P}_{2}-w^{3} \mathrm{D}_{1}^{\circ}\end{array}$ & $\begin{array}{r}5 \\
2 \\
50 \\
10 \\
60\end{array}$ & $\begin{array}{l}\text { 2910. } 936 \\
2911.148 \\
2912.433 \\
2912.745 \\
2913.163\end{array}$ & $\begin{array}{l}34343.15 \\
34340.65 \\
34325.50 \\
34321.83 \\
34316.90\end{array}$ & $\begin{array}{l}b{ }^{3} \mathrm{~F}_{2}-w^{5} \mathrm{D}_{2}^{\circ} \\
a^{3} \mathrm{G}_{4}-17_{4}^{\circ} \\
a^{3} \mathrm{~F}_{2}-u^{3} \mathrm{D}_{2}^{\circ} \\
a^{5} \mathrm{D}_{3}-v^{3} \mathrm{D}_{2}^{\circ} \\
a^{3} \mathrm{P}_{1}-11_{0}^{\circ}\end{array}$ \\
\hline $\begin{array}{r}2 \\
40 \\
40 \\
100 \\
50\end{array}$ & $\begin{array}{l}2868.945 \\
2870.213 \\
2871.186 \\
2871.642 \\
2873.370\end{array}$ & $\begin{array}{l}34845.79 \\
34830.40 \\
34818.59 \\
34813.07 \\
34792.13\end{array}$ & $\begin{array}{l}a{ }^{5} \mathrm{D}_{2}-y^{1} \mathrm{D}_{2}^{\circ} \\
a^{5} \mathrm{D}_{1}-y^{1} \mathrm{D}_{2}^{\circ} \\
a^{3} \mathrm{G}_{4}-23_{3}^{\circ} \\
a^{3} \mathrm{~F}_{3}-v^{3} \mathrm{D}_{2}^{\circ} \\
a^{3}{ }^{3} \mathrm{~F}_{2}-x^{3} \mathrm{G}_{3}^{\circ}\end{array}$ & $\begin{array}{r}50 \\
25 \\
150 \\
40 \\
50\end{array}$ & $\begin{array}{l}\text { 2914. } 294 \\
2915.614 \\
2916.251 \\
2917.132 \\
2917.764\end{array}$ & $\begin{array}{l}34303.58 \\
34288.05 \\
34280.56 \\
34270.21 \\
34262.79\end{array}$ & $\begin{array}{l}a{ }^{3} \mathrm{P}_{0}-9_{i}^{\circ} \\
a^{3} \mathrm{G}_{5}-x^{1} \mathrm{H}_{5}^{\circ} \\
a \\
{ }^{5} \mathrm{~F}_{4}-y^{5} \mathrm{~F}_{4}^{\circ} \\
a \\
a^{3} \mathrm{P}_{1}-9_{1}^{\circ} \\
a^{3} \mathrm{~F}_{5}-x^{3} \mathrm{~F}_{4}^{\circ}\end{array}$ \\
\hline $\begin{array}{c}25 \\
1000 R \\
10 \\
40 \\
1\end{array}$ & $\begin{array}{l}2874.050 \\
2874.984 \\
2875.316 \\
2877.092 \\
2877.339\end{array}$ & $\begin{array}{l}34783.90 \\
34772.60 \\
34768.59 \\
34747.12 \\
34744.14\end{array}$ & $\begin{array}{ll}a & { }^{5} \mathrm{D}_{2}-u^{3} \mathrm{D}_{\mathrm{i}}^{\circ} \\
a & { }^{5} \mathrm{~F}_{5}-y{ }^{5} \mathrm{~F}_{5}^{\circ} \\
a & { }^{5} \mathrm{D}_{1}-u^{3} \mathrm{D}_{\mathrm{i}}^{\circ} \\
a & { }^{3} \mathrm{P}_{0}-t^{3} \mathrm{D}_{1}^{\circ} \\
b & { }^{3} \mathrm{~F}_{2}-w^{5} \mathrm{D}_{1}^{\circ}\end{array}$ & $\begin{array}{l}75 \\
20 \\
1 H \\
30 \\
3\end{array}$ & $\begin{array}{l}2919.604 \\
2920.254 \\
2920.765 \\
2920.949 \\
2921.140\end{array}$ & $\begin{array}{l}34241.20 \\
34233.58 \\
34227.59 \\
34225.43 \\
34223.19\end{array}$ & $\begin{array}{l}a a^{5} \mathrm{~F}_{1}-z{ }^{3} \mathrm{P}_{1}^{\circ} \\
a^{1} \mathrm{G}_{4}-43_{3}^{\circ} \\
a \\
{ }^{5} \mathrm{P}_{3}-y^{1} \mathrm{~F}_{3}^{\circ} \\
a^{3} \mathrm{P}_{0}-8_{1}^{\circ} \\
a^{3}{ }^{3} \mathrm{~F}_{4}-x^{3} \mathrm{D}_{3}^{\circ}\end{array}$ \\
\hline $\begin{array}{r}1 \\
50 \\
25 \\
100 \\
10\end{array}$ & $\begin{array}{l}2877.489 \\
2877.826 \\
2879.358 \\
2879.756 \\
2880.227\end{array}$ & $\begin{array}{l}34742.33 \\
34738.26 \\
34719.78 \\
34714.98 \\
34709.30\end{array}$ & $\begin{array}{ll}b & { }^{3} \mathrm{~F}_{4}-w^{3} \mathrm{G}_{4} \\
a & { }^{5} \mathrm{P}_{3}-u^{3} \mathrm{D}_{2}^{\circ} \\
a^{3} \mathrm{~F}_{2}-y & { }^{1} \mathrm{D}_{2}^{\circ} \\
a^{3} \mathrm{~F}_{4}-x^{3} \mathrm{~F}_{3}^{\circ} \\
a^{3} \mathrm{G}_{4}-22_{3}^{\circ}\end{array}$ & $\begin{array}{r}3 \\
1 \\
5 \\
20 \\
2\end{array}$ & $\begin{array}{l}\text { 2921. } 406 \\
2923.677 \\
2923.804 \\
2925.067 \\
2925.748\end{array}$ & $\begin{array}{l}34220.08 \\
34193.50 \\
34192.01 \\
34177.25 \\
34169.30\end{array}$ & $\begin{array}{l}b{ }^{3} \mathrm{P}_{0}-45_{1}^{\circ} \\
a^{3} \mathrm{G}_{5}-13_{4}^{\circ} \\
a^{3} \mathrm{P}_{1}-8_{1}^{\circ} \\
a^{3} \mathrm{P}_{2}-x^{5} \mathrm{~F}_{3}^{\circ} \\
a^{3} \mathrm{G}_{3}-28_{3}^{\circ}\end{array}$ \\
\hline $\begin{array}{r}2 \\
60 \\
15 \\
5 \\
75\end{array}$ & $\begin{array}{l}2880.506 \\
2881.273 \\
2882.577 \\
2882.622 \\
2883.594\end{array}$ & $\begin{array}{l}34705.94 \\
34696.70 \\
34681.01 \\
34680.47 \\
34668.78\end{array}$ & $\begin{array}{l}a^{3} \mathrm{G}_{3}-34_{3}^{\circ} \\
a^{5} \mathrm{~F}_{1}-x{ }^{5} \mathrm{D}_{0}^{\circ} \\
b^{3} \mathrm{P}_{2}-33_{2}^{\circ} \\
a^{5} \mathrm{P}_{1}-3_{1}^{\circ} \\
a^{3} \mathrm{P}_{1}-w^{5} \mathrm{D}_{0}^{\circ} \\
a^{5} \mathrm{~F}_{3}-y^{3} \mathrm{D}_{3}^{\circ}\end{array}$ & $\begin{array}{r}5 \\
60 \\
50 \\
1 \\
40\end{array}$ & $\begin{array}{l}\text { 2925. } 841 \\
2927.119 \\
2928.487 \\
2929.122 \\
2929.434\end{array}$ & $\begin{array}{l}34168.21 \\
34153.29 \\
34137.34 \\
34129.94 \\
34126.30\end{array}$ & $\begin{array}{ll}a & { }^{5} \mathrm{P}_{3}-x^{3} \mathrm{G}_{4} \\
a & { }^{1} \mathrm{G}_{4}-42_{4}^{\circ} \\
a & { }^{3} \mathrm{P}_{1}-t^{3} \mathrm{D}_{2}^{\circ} \\
a^{3} \mathrm{G}_{4}-w^{3} \mathrm{~F}_{3}^{\circ} \\
a^{5} \mathrm{P}_{3}-v^{3} \mathrm{D}_{2}^{\circ}\end{array}$ \\
\hline $\begin{array}{r}50 \\
80 \\
150 \\
70 \\
50\end{array}$ & $\begin{array}{l}2884.500 \\
2884.843 \\
2886.528 \\
2887.993 \\
2888.624\end{array}$ & $\begin{array}{l}34657.89 \\
34653.77 \\
34633.54 \\
34615.97 \\
34608.41\end{array}$ & 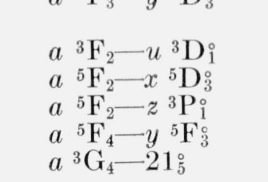 & $\begin{array}{l}5 H \\
75 \\
2 \\
60 \\
30\end{array}$ & $\begin{array}{l}\text { 2932. } 593 \\
2934.173 \\
2934.658 \\
2936.005 \\
2936.247\end{array}$ & $\begin{array}{l}34089.54 \\
34071.19 \\
34065.56 \\
34049.93 \\
34047.12\end{array}$ & $\begin{array}{l}a^{3}{ }^{3} \mathrm{G}_{3}-25_{3}^{\circ} \\
a{ }^{3} \mathrm{~F}_{4}-z^{1} \mathrm{H}^{\circ} \\
a^{1} \mathrm{G}_{4}-40_{3}^{\circ} \\
a^{5} \mathrm{D}_{2}-y^{3} \mathrm{~S}_{1}^{\circ} \\
a^{5} \mathrm{~F}_{2}-y^{3} \mathrm{D}_{3}^{\circ}\end{array}$ \\
\hline $\begin{array}{l}1 h \\
10 \\
50 \\
60 \\
10\end{array}$ & $\begin{array}{l}2889.047 \\
2890.879 \\
2891.130 \\
2891.645 \\
2892.470\end{array}$ & $\begin{array}{l}34603.34 \\
34581.42 \\
34578.41 \\
34572.26 \\
34562.39\end{array}$ & $\begin{array}{l}a^{1} \mathrm{G}_{4}-48_{3}^{\circ} \\
b^{3} \mathrm{P}_{0}-49_{1}^{\circ} \\
a^{3} \mathrm{P}_{2}-x^{1} \mathrm{~F}_{3}^{\circ} \\
a^{3} \mathrm{H}_{6}-v^{3} \mathrm{G}_{5}^{\circ} \\
a^{5} \mathrm{~F}_{1}-x^{5} \mathrm{D}_{2}^{\circ}\end{array}$ & $\begin{array}{r}30 \\
1 \\
75 \\
25 \\
80\end{array}$ & $\begin{array}{l}2937.336 \\
2937.850 \\
2939.135 \\
2939.676 \\
2939.938\end{array}$ & $\begin{array}{l}34034.50 \\
34028.54 \\
34013.67 \\
34007.41 \\
34004.38\end{array}$ & $\begin{array}{ll}a & { }^{5} \mathrm{D}_{1}-y{ }^{3} \mathrm{~S}_{i} \\
b & { }^{3} \mathrm{~F}_{2}-6_{3}^{\circ} \\
a & { }^{5} \mathrm{~F}_{1}-z^{3} \mathrm{P}_{2}^{\circ} \\
b & { }^{3} \mathrm{~F}_{3}-y^{3} \mathrm{H}_{4}^{\circ} \\
a^{3} \mathrm{P}_{1}-w^{5} \mathrm{D}_{2}^{\circ}\end{array}$ \\
\hline $\begin{array}{r}20 \\
15 \\
100 \\
50 \\
3\end{array}$ & $\begin{array}{l}2893.731 \\
2895.802 \\
2896.523 \\
2898.533 \\
2898.715\end{array}$ & $\begin{array}{l}34547.34 \\
34522.63 \\
34514.04 \\
34490.10 \\
34487.94\end{array}$ & $\begin{array}{l}b{ }^{3} \mathrm{~F}_{3}-x{ }^{1} \mathrm{~F}_{3}^{\circ} \\
a^{3} \mathrm{G}_{4}-s^{3} \mathrm{D}_{3}^{\circ} \\
a^{5} \mathrm{~F}_{1}-y^{3} \mathrm{D}_{1}^{\circ} \\
a a^{5} \mathrm{P}_{2}-w^{3} \mathrm{D}_{2}^{\circ} \\
a^{3} \mathrm{G}_{5}-14_{4}^{\circ}\end{array}$ & $\begin{array}{r}100 \\
1 \\
10 \\
60 \\
100\end{array}$ & $\begin{array}{l}2940.352 \\
2941.212 \\
2941.762 \\
2943.470 \\
2943.919\end{array}$ & $\begin{array}{l}33999.59 \\
33989.65 \\
33983.30 \\
33963.58 \\
33958.40\end{array}$ & $\begin{array}{l}a^{5}{ }^{5} \mathrm{D}_{4}-w^{3} \mathrm{D}_{3}^{\circ} \\
b \\
{ }^{3} \mathrm{P}_{2}-23_{3}^{\circ} \\
a^{3} \mathrm{D}_{2}-44_{2}^{\circ} \\
a{ }^{5} \mathrm{P}_{2}-x{ }^{3} \mathrm{D}_{2}^{\circ} \\
a^{5} \mathrm{D}_{3}-w^{3} \mathrm{D}_{2}^{\circ}\end{array}$ \\
\hline $\begin{array}{r}50 \\
1 \\
25 \\
50\end{array}$ & $\begin{array}{l}\text { 2899. } 716 \\
\text { 2900. } 669 \\
2901.784 \\
2901.937\end{array}$ & $\begin{array}{l}34476.04 \\
34464.71 \\
34451.47 \\
34449.65\end{array}$ & $\begin{array}{l}b^{3} \mathrm{~F}_{2}-t^{3} \mathrm{D}_{2}^{\circ} \\
b^{3} \mathrm{~F}_{4}-y^{1} \mathrm{H}_{5}^{\circ} \\
a^{1} \mathrm{G}_{4}-47_{4}^{\circ} \\
a^{5} \mathrm{D}_{2}-u^{3} \mathrm{D}_{2}^{\circ} \\
a^{3} \mathrm{~F}_{3}-w^{3} \mathrm{D}_{2}^{\circ}\end{array}$ & $\begin{array}{r}10 \\
100 \\
2 \\
150 \\
2\end{array}$ & $\begin{array}{l}\text { 2944. } 380 \\
2946.981 \\
2948.847 \\
2949.492 \\
2949.947\end{array}$ & $\begin{array}{l}33953.08 \\
33923.12 \\
33901.65 \\
33894.24 \\
33889.01\end{array}$ & $\begin{array}{ll}b & { }^{3} \mathrm{~F}_{3}-x{ }^{5} \mathrm{~F}_{4}^{\circ} \\
a & 3 \mathrm{~F}_{3}-x^{3} \mathrm{D}_{2}^{\circ} \\
b & { }^{3} \mathrm{P}_{2}-s^{3} \mathrm{D}_{2}^{\circ} \\
a & { }^{3} \mathrm{~F}_{4}-1_{4}^{\circ} \\
a & { }^{5} \mathrm{P}_{1}-u^{3} \mathrm{D}_{2}^{\circ}\end{array}$ \\
\hline $\begin{array}{r}50 \\
25 \\
40 \\
5 \\
10\end{array}$ & $\begin{array}{l}\text { 2902. } 087 \\
2902.854 \\
2903.074 \\
2904.191 \\
2904.704\end{array}$ & $\begin{array}{l}34447.87 \\
34438.77 \\
34436.16 \\
34422.91 \\
34416.83\end{array}$ & $\begin{array}{ll}a & { }^{3} \mathrm{P}_{2}-w^{5} \mathrm{D}_{3}^{\circ} \\
a & { }^{3} \mathrm{P}_{0}-w^{5} \mathrm{D}_{1}^{\circ} \\
a & { }^{5} \mathrm{D}_{1}-u^{3} \mathrm{D}_{2}^{\circ} \\
a^{5} \mathrm{D}_{3}-y^{1} \mathrm{~F}_{3}^{\circ} \\
b^{3} \mathrm{~F}_{3}-w^{5} \mathrm{D}_{3}^{\circ}\end{array}$ & $\begin{array}{r}50 \\
60 \\
80 \\
1 \\
125\end{array}$ & $\begin{array}{l}2950.532 \\
2951.401 \\
2952.489 \\
2952.931 \\
2954.484\end{array}$ & $\begin{array}{l}33882.29 \\
33872.31 \\
33859.84 \\
33854.77 \\
33836.97\end{array}$ & $\begin{array}{ll}a{ }^{3} \mathrm{H}_{5}-v^{3} \mathrm{G}_{5}^{\circ} \\
a & 5 \mathrm{~F}_{3}-y{ }^{5} \mathrm{~F}_{2}^{\circ} \\
a & 5 \mathrm{~F}_{1}-y^{3} \mathrm{D}_{2}^{\circ} \\
b & { }^{3} \mathrm{P}_{2}-x^{5} \mathrm{P}_{1}^{\circ} \\
a^{5} \mathrm{D}_{2}-w^{3} \mathrm{D}_{1}^{\circ}\end{array}$ \\
\hline
\end{tabular}


Table 3-Continued

\begin{tabular}{|c|c|c|c|c|c|c|c|}
\hline Intensity & $\begin{array}{l}\text { Wave- } \\
\text { length }\end{array}$ & $\begin{array}{c}\text { Wave } \\
\text { number }\end{array}$ & Term combination & Intensity & $\begin{array}{l}\text { Wave- } \\
\text { length }\end{array}$ & $\begin{array}{c}\text { Wave } \\
\text { number }\end{array}$ & Term combination \\
\hline $\begin{array}{r}50 \\
20 \\
20 \\
5 \\
4\end{array}$ & $\begin{array}{c}A \\
2955.348 \\
2955.593 \\
2955.833 \\
2956.133 \\
2957.166\end{array}$ & $\begin{array}{c}\mathrm{cm}^{-1} \\
33827.08 \\
33824.28 \\
33821.53 \\
33818.10 \\
33806.28\end{array}$ & $\begin{array}{l}a^{3} \mathrm{G}_{3}-22_{3}^{\circ} \\
a^{5} \mathrm{D}_{1}-v^{3} \mathrm{D}_{2}^{\circ} \\
a^{5} \mathrm{D}_{1}-w^{3} \mathrm{D}_{1}^{\circ} \\
a^{3} \mathrm{P}_{2}-4_{3}^{\circ} \\
a^{3} \mathrm{P}_{1}-x^{5} \mathrm{~F}_{2}^{\circ}\end{array}$ & $\begin{array}{l}2 h \\
75 \\
75 \\
1 h \\
20\end{array}$ & $\begin{array}{c}\text { A } \\
3007.88 \\
3008.262 \\
3008.797 \\
3009.537 \\
3009.687\end{array}$ & $\begin{array}{c}c m^{-1} \\
33236.32 \\
33232.10 \\
33226.19 \\
33218.02 \\
33216.37\end{array}$ & $\begin{array}{ll}a & { }^{5} \mathrm{P}_{3}-x^{3} \mathrm{D}_{2}^{\circ} \\
a & { }^{3} \mathrm{~F}_{2}-z{ }^{1} \mathrm{P}_{1}^{\circ} \\
b & { }^{3} \mathrm{~F}_{4}-x^{3} \mathrm{~F}_{4}^{\circ} \\
a & { }^{3} \mathrm{P}_{2}-u^{3} \mathrm{D}_{1}^{\circ} \\
a^{5}{ }^{5} \mathrm{P}_{2}-x^{3} \mathrm{~F}_{3}\end{array}$ \\
\hline $\begin{array}{r}100 \\
2 \\
100 \\
40\end{array}$ & $\begin{array}{l}\text { 2957. } 996 \\
2958.858 \\
2959.729 \\
2960.965\end{array}$ & $\begin{array}{l}33796.80 \\
33786.95 \\
33777.01 \\
33762.91\end{array}$ & $\begin{array}{l}a{ }^{3} \mathrm{~F}_{3}-v^{3} \mathrm{D}_{3}^{\circ} \\
b^{3} \mathrm{~F}_{3}-4_{3}^{\circ} \\
a^{5} \mathrm{D}_{4}-x^{3} \mathrm{~F}_{3}^{\circ} \\
b^{3} \mathrm{P}_{0}-39_{1}^{\circ} \\
a^{5} \mathrm{P}_{3}-w^{3} \mathrm{D}_{2}^{\circ}\end{array}$ & $\begin{array}{r}40 \\
3 \\
75 \\
75 \\
1\end{array}$ & $\begin{array}{l}3010.501 \\
3011.607 \\
3012.915 \\
3013.354 \\
3014.070\end{array}$ & $\begin{array}{l}33207.39 \\
33195.19 \\
33180.78 \\
33175.95 \\
33168.07\end{array}$ & $\begin{array}{l}a{ }^{3} \mathrm{H}_{5}-51_{4}^{\circ} \\
b^{3} \mathrm{P}_{1}-18_{0}^{\circ} \\
a^{5} \mathrm{D}_{3}-z^{1} \mathrm{D}_{2}^{\circ} \\
a^{3} \mathrm{~F}_{3}-x^{3} \mathrm{~F}_{3}^{\circ} \\
a^{1}{ }^{1} \mathrm{G}_{4}-28_{3}^{\circ}\end{array}$ \\
\hline $\begin{array}{r}1 \\
75 \\
100 \\
15 \\
150\end{array}$ & $\begin{array}{l}\text { 2961. } 334 \\
\text { 2961. } 685 \\
\text { 2963. } 714 \\
\text { 2964. } 295 \\
\text { 2965. } 168\end{array}$ & $\begin{array}{l}33758.70 \\
33754.70 \\
33731.59 \\
33724.98 \\
33715.06\end{array}$ & $\begin{array}{l}a^{3} \mathrm{D}_{3}-v^{3} \mathrm{G}_{4}^{\circ} \\
b{ }^{3} \mathrm{~F}_{2}-x{ }^{1} \mathrm{~F}_{3}^{\circ} \\
a \mathrm{~F}_{4}^{3}-y^{3} \mathrm{G}_{4}^{\circ} \\
a^{3} \mathrm{D}_{2}-41_{2}^{\circ} \\
a^{5} \mathrm{~F}_{3}-y{ }^{5} \mathrm{~F}_{3}^{\circ}\end{array}$ & $\begin{array}{r}1 \\
2 \\
200\end{array}$ & $\begin{array}{l}3015.008 \\
3016.684 \\
3017.235\end{array}$ & $\begin{array}{l}33157.75 \\
33139.33 \\
33133.28\end{array}$ & $\begin{array}{l}a^{3} \mathrm{G}_{5}-y^{1} \mathrm{G}_{4}^{\circ} \\
b^{3} \mathrm{P}_{2}-x^{5} \mathrm{P}_{2}^{\circ} \\
a^{5} \mathrm{P}_{2}-x^{3} \mathrm{~F}_{2}^{\circ} \\
a^{5} \mathrm{~F}_{1}-y^{5} \mathrm{~F}_{1} \\
a^{5} \mathrm{D}_{4}-z^{1} \mathrm{H}_{5}^{\circ}\end{array}$ \\
\hline $\begin{array}{r}15 \\
20 \\
1 \\
50 \\
30\end{array}$ & $\begin{array}{l}2965.694 \\
2966.549 \\
2967.064 \\
2967.342 \\
2968.398\end{array}$ & $\begin{array}{l}33709.08 \\
33699.36 \\
33693.51 \\
33690.36 \\
33678.37\end{array}$ & $\begin{array}{l}a^{3} \mathrm{H}_{5}-v^{3} \mathrm{G}_{4}^{\circ} \\
b{ }^{3} \mathrm{P}_{2}-20_{2}^{\circ} \\
b{ }^{3} \mathrm{P}_{2}-s^{3} \mathrm{D}_{3}^{\circ} \\
a^{3} \mathrm{~F}_{4}-y{ }^{3} \mathrm{G}_{3}^{\circ} \\
a^{3} \mathrm{G}_{4}-x{ }^{1} \mathrm{H}_{5}^{\circ}\end{array}$ & $\begin{array}{c}5 \\
15 \\
1 \mathrm{w} \\
150 \\
20\end{array}$ & $\begin{array}{l}\text { 3019. } 291 \\
3019.367 \\
3020.218 \\
3020.871 \\
3025.085\end{array}$ & $\begin{array}{l}33110.71 \\
33109.88 \\
33100.55 \\
33093.40 \\
33047.30\end{array}$ & 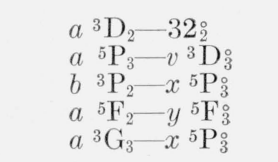 \\
\hline $\begin{array}{r}30 \\
100 \\
2 \\
1 \\
25\end{array}$ & $\begin{array}{l}\text { 2968. } 468 \\
2968.952 \\
2969.854 \\
2971.261 \\
2971.757\end{array}$ & $\begin{array}{l}33677.58 \\
33672.09 \\
33661.86 \\
33645.92 \\
33640.30\end{array}$ & $\begin{array}{l}a{ }^{3} \mathrm{P}_{2}-3_{1}^{\circ} \\
a^{3} \mathrm{~F}_{3}-z^{1} \mathrm{D}_{2}^{\circ} \\
a^{1} \mathrm{H}_{5}-62_{4}^{\circ} \\
a^{3} \mathrm{G}_{3}-20_{2}^{\circ} \\
a^{3} \mathrm{G}_{3}-s^{3} \mathrm{D}_{3}^{\circ}\end{array}$ & $\begin{array}{r}60 \\
5 \\
1 \\
60 \\
10\end{array}$ & $\begin{array}{l}3027.076 \\
3029.373 \\
3029.948 \\
3030.776 \\
3031.107\end{array}$ & $\begin{array}{l}33025.56 \\
33000.52 \\
32994.26 \\
32985.25 \\
32981.65\end{array}$ & $\begin{array}{l}a^{3} \mathrm{H}_{4}-v^{3} \mathrm{G}_{5}^{\circ} \\
a{ }^{5} \mathrm{P}_{1}-z^{1} \mathrm{~S}_{0}^{\circ} \\
b{ }^{3} \mathrm{~F}_{2}-4_{3}^{\circ} \\
a{ }^{5} \mathrm{P}_{3}-z^{1} \mathrm{D}_{2}^{\circ} \\
b^{3} \mathrm{P}_{0}-26_{1}^{\circ}\end{array}$ \\
\hline $\begin{array}{r}1 \\
100 \\
30 \\
25 \\
100\end{array}$ & $\begin{array}{l}2972.98 \\
2973.976 \\
2974.327 \\
2975.122 \\
2976.923\end{array}$ & $\begin{array}{l}33626.47 \\
33615.21 \\
33611.24 \\
33602.26 \\
33581.93\end{array}$ & $\begin{array}{l}a^{3} \mathrm{D}_{1}-58_{1}^{\circ} \\
a^{5} \mathrm{D}_{0}-y{ }^{3} s_{1}^{\circ} \\
a{ }^{3} \mathrm{P}_{2}-x^{3} \mathrm{P}_{2}^{\circ} \\
b{ }^{3} \mathrm{P}_{2}-w^{3} \mathrm{~F}_{2}^{\circ} \\
a^{5} \mathrm{~F}_{4}-y{ }^{5} \mathrm{~F}_{5}^{\circ}\end{array}$ & $\begin{array}{r}50 \\
100 \\
50 \\
50 \\
40\end{array}$ & $\begin{array}{l}3031.899 \\
3033.449 \\
3034.058 \\
3035.471 \\
3036.463\end{array}$ & $\begin{array}{l}32973.03 \\
32956.18 \\
32949.57 \\
32934.23 \\
32923.47\end{array}$ & $\begin{array}{l}a{ }^{5} \mathrm{P}_{2}-x^{3} \mathrm{D}_{1}^{\circ} \\
a^{5} \mathrm{D}_{4}-1_{4}^{\circ} \\
a^{5} \mathrm{D}_{2}-x^{3} \mathrm{D}_{2}^{\circ} \\
a^{5} \mathrm{D}_{1}-x^{3} \mathrm{D}_{2}^{\circ} \\
a^{5} \mathrm{D}_{0}-z^{1} \mathrm{P}_{1}\end{array}$ \\
\hline $\begin{array}{l}30 \\
60 \\
75 \\
10 \\
20\end{array}$ & $\begin{array}{l}\text { 2978. } 361 \\
2979.713 \\
2981.934 \\
2982.522 \\
2985.812\end{array}$ & $\begin{array}{l}33565.72 \\
33550.49 \\
33525.50 \\
33518.89 \\
33481.96\end{array}$ & $\begin{array}{l}b{ }^{3} \mathrm{P}_{1}-s^{3} \mathrm{D}_{2}^{\circ} \\
a^{3} \mathrm{D}_{2}-39_{1}^{\circ} \\
a{ }^{5} \mathrm{~F}_{2}-y{ }^{5} \mathrm{~F}_{1}^{\circ} \\
b{ }^{3} \mathrm{P}_{1}-x^{5} \mathrm{P}_{1}^{\circ} \\
a^{3} \mathrm{H}_{5}-52_{4}^{\circ}\end{array}$ & $\begin{array}{r}5 \\
40 \\
50 \\
10 \\
25\end{array}$ & $\begin{array}{l}3037.376 \\
3037.961 \\
3038.169 \\
3039.674 \\
3039.952\end{array}$ & $\begin{array}{l}32913.58 \\
32907.24 \\
32904.99 \\
32888.70 \\
32885.69\end{array}$ & $\begin{array}{ll}a & 5 \mathrm{P}_{1}-w^{3} \mathrm{D}_{2}^{\circ} \\
a & { }^{5} \mathrm{D}_{3}-w^{3} \mathrm{D}_{3}^{\circ} \\
a & { }^{5} \mathrm{P}_{2}-z^{1} \mathrm{~F}_{3}^{\circ} \\
a & { }^{3} \mathrm{~F}_{4}-y^{3} \mathrm{~F}_{3}^{\circ} \\
a^{3} \mathrm{P}_{2}-u^{3} \mathrm{D}_{2}^{\circ}\end{array}$ \\
\hline $\begin{array}{r}25 \\
2 \\
30 \\
6 \\
10\end{array}$ & $\begin{array}{l}\text { 2986. } 328 \\
2987.269 \\
2987.694 \\
2987.928 \\
2988.089\end{array}$ & $\begin{array}{l}33476.18 \\
33465.63 \\
33460.87 \\
33458.25 \\
33456.45\end{array}$ & $\begin{array}{l}a^{5} \mathrm{D}_{2}-w^{3} \mathrm{D}_{2}^{\circ} \\
a^{5} \mathrm{D}_{4}-z^{1} \mathrm{~F}_{3}^{\circ} \\
a^{5} \mathrm{D}_{1}-w^{3} \mathrm{D}_{2}^{\circ} \\
a^{3} \mathrm{G}_{3}-17_{4}^{\circ} \\
a^{3} \mathrm{G}_{4}-12_{4}^{\circ}\end{array}$ & $\begin{array}{r}100 \\
40 \\
100 \\
60 \\
20\end{array}$ & $\begin{array}{l}3040.319 \\
3041.906 \\
3042.480 \\
3042.829 \\
3043.045\end{array}$ & $\begin{array}{l}32881.72 \\
32864.56 \\
32858.36 \\
32854.59 \\
32852.26\end{array}$ & $\begin{array}{ll}a & 5 \mathrm{~F}_{4}-z^{5} \mathrm{P}_{3}^{\circ} \\
a & { }^{3} \mathrm{~F}_{3}-z^{1} \mathrm{~F}_{3}^{\circ} \\
a & 5 \mathrm{~F}_{1}-y^{5} \mathrm{~F}_{2}^{\circ} \\
b & { }^{3} \mathrm{~F}_{3}-u^{3} \mathrm{D}_{2}^{\circ} \\
a^{3} \mathrm{H}_{4}-v^{3} \mathrm{G}_{4}^{\circ}\end{array}$ \\
\hline $\begin{array}{r}300 \\
50 \\
40 \\
40\end{array}$ & $\begin{array}{l}\text { 2988. } 945 \\
\text { 2989. } 652 \\
\text { 2990. } 289 \\
\text { 2992. } 120\end{array}$ & $\begin{array}{l}33446.86 \\
33438.95 \\
33431.83 \\
33411.37\end{array}$ & $\begin{array}{l}a{ }^{5} \mathrm{~F}_{5}-y{ }^{5} \mathrm{D}_{4}^{\circ} \\
a{ }^{5} \mathrm{P}_{2}-w^{3} \mathrm{D}_{3}^{\circ} \\
a^{3} \mathrm{D}_{2}-35_{3}^{\circ} \\
a^{5} \mathrm{D}_{3}-x^{3} \mathrm{D}_{2}^{\circ} \\
a^{3} \mathrm{P}_{1}-y^{5} s_{2}^{\circ}\end{array}$ & $\begin{array}{r}1 h \\
125 \\
2 \\
1\end{array}$ & $\begin{array}{l}3043.553 \\
3045.715 \\
3047.187 \\
3048.30\end{array}$ & $\begin{array}{l}32846.78 \\
32823.47 \\
32807.61 \\
32795.63\end{array}$ & $\begin{array}{l}a^{3} \mathrm{D}_{3}-44_{2}^{\circ} \\
a^{3} \mathrm{~F}_{2}-x^{3} \mathrm{D}_{2}^{\circ} \\
a^{5} \mathrm{D}_{2}-v^{3} \mathrm{D}_{3}^{\circ} \\
b^{3} \mathrm{P}_{1}-15_{1}^{\circ} \\
a^{5} \mathrm{P}_{1}-z^{1} \mathrm{P}_{1}^{\circ}\end{array}$ \\
\hline $\begin{array}{r}30 \\
100 \\
150 \\
75 \\
50\end{array}$ & $\begin{array}{l}\text { 2992. } 948 \\
\text { 2993. } 273 \\
\text { 2994. } 967 \\
2996.891 \\
2997.421\end{array}$ & $\begin{array}{l}33402.13 \\
33398.51 \\
33379.62 \\
33358.19 \\
33352.29\end{array}$ & $\begin{array}{l}a{ }^{5} \mathrm{D}_{0}-w^{3} \mathrm{D}_{1}^{\circ} \\
a^{3} \mathrm{~F}_{3}-w^{3} \mathrm{D}_{3}^{\circ} \\
a{ }^{5} \mathrm{~F}_{3}-y^{5} \mathrm{~F}_{4}^{\circ} \\
a^{5} \mathrm{D}_{2}-z^{1} \mathrm{P}_{1}^{\circ} \\
a^{3} \mathrm{P}_{2}-x^{3} \mathrm{G}_{3}^{\circ}\end{array}$ & $\begin{array}{r}75 \\
150 \\
2 \\
50 \\
1\end{array}$ & $\begin{array}{l}3048.496 \\
3048.793 \\
3049.066 \\
3051.593 \\
3052.075\end{array}$ & $\begin{array}{l}32793.52 \\
32790.33 \\
32787.39 \\
32760.24 \\
32755.07\end{array}$ & $\begin{array}{l}a{ }^{5} \mathrm{D}_{4}-y^{3} \mathrm{G}_{4}^{\circ} \\
a{ }^{5} \mathbf{F}_{3}-z^{5} \mathbf{P}_{2}^{\circ} \\
b{ }^{3} \mathbf{F}_{2}-x^{3} \mathbf{P}_{2}^{\circ} \\
b{ }^{3} \mathbf{F}_{4}-v^{3} \mathrm{D}_{3}^{\circ} \\
a^{3} \mathrm{D}_{2}-26_{1}^{\circ}\end{array}$ \\
\hline $\begin{array}{r}50 \\
60 \\
60 \\
5 \\
20\end{array}$ & $\begin{array}{l}\text { 2997. } 613 \\
3000.217 \\
3001.634 \\
3002.050 \\
3003.473\end{array}$ & $\begin{array}{l}33350.15 \\
33321.21 \\
33305.48 \\
33300.86 \\
33285.09\end{array}$ & $\begin{array}{ll}a & { }^{3} \mathrm{~F}_{2}-w^{3} \mathrm{D}_{2}^{\circ} \\
b & { }^{3} \mathrm{~F}_{3}-x^{3} \mathrm{G}_{3}^{\circ} \\
a^{5} \mathrm{D}_{3}-v^{3} \mathrm{D}_{3}^{\circ} \\
b^{3} \mathrm{P}_{2}-w^{3} \mathrm{~F}_{3}^{\circ} \\
a^{5}{ }^{5} \mathrm{D}_{4}-x^{3} \mathrm{D}_{3}^{\circ}\end{array}$ & $\begin{array}{r}15 \\
3 \\
150 \\
30\end{array}$ & $\begin{array}{l}3052.334 \\
3054.563 \\
3054.934 \\
3056.108\end{array}$ & $\begin{array}{l}32752.29 \\
32728.39 \\
32724.42 \\
32711.84\end{array}$ & $\begin{array}{ll}a & { }^{5} \mathrm{D}_{4}-y^{3} \mathrm{G}_{3}^{\circ} \\
a & { }^{3} \mathrm{~F}_{4}-z^{3} \mathrm{H}_{4}^{\circ} \\
a & 5 \mathrm{P}_{2}-x^{3} \mathrm{D}_{3}^{\circ} \\
a & 5 \mathrm{P}_{3}-w^{3} \mathrm{D}_{3}^{\circ} \\
a^{5} & { }^{3} \mathrm{G}_{4}-x{ }^{1} \mathrm{G}_{4}^{\circ}\end{array}$ \\
\hline $\begin{array}{r}3 \\
15 \\
50 \\
300\end{array}$ & $\begin{array}{l}3003.939 \\
3004.211 \\
3004.598 \\
3006.588\end{array}$ & $\begin{array}{l}33279.92 \\
33276.91 \\
33272.62 \\
33250.60\end{array}$ & $\begin{array}{l}a{ }^{3} \mathrm{P}_{2}-y^{1} \mathrm{D}_{2}^{\circ} \\
a{ }^{5} \mathrm{P}_{1}-v^{3} \mathrm{D}_{2}^{\circ} \\
a^{3} \mathrm{D}_{2}-33_{2}^{\circ} \\
a^{5} \mathrm{~F}_{2}-y^{5} \mathrm{~F}_{2}^{\circ} \\
a^{3} \mathrm{G}_{4}-10_{4}^{\circ}\end{array}$ & $\begin{array}{r}8 \\
50 \\
75 \\
75 \\
1\end{array}$ & $\begin{array}{l}3056.754 \\
3057.353 \\
3058.650 \\
3058.795 \\
3059.733\end{array}$ & $\begin{array}{l}32704.93 \\
32698.52 \\
32684.66 \\
32683.11 \\
32673.09\end{array}$ & $\begin{array}{l}a^{3} \mathrm{D}_{1}-50_{2}^{\circ} \\
a^{5} \mathrm{D}_{2}-z^{1} \mathrm{D}_{2}^{\circ} \\
a^{5} \mathrm{D}_{3}-x^{3} \mathrm{~F}_{3}^{\circ} \\
a^{5} \mathrm{D}_{1}-z^{1} \mathrm{D}_{2}^{\circ} \\
b^{3} \mathrm{P}_{0}-x^{5} \mathrm{P}_{1}^{\circ}\end{array}$ \\
\hline
\end{tabular}


Table 3-Continued

\begin{tabular}{|c|c|c|c|c|c|c|c|}
\hline Intensity & $\begin{array}{l}\text { Wave- } \\
\text { length }\end{array}$ & $\begin{array}{c}\text { Wave } \\
\text { number }\end{array}$ & Term combination & Intensity & $\begin{array}{l}\text { Wave- } \\
\text { length }\end{array}$ & $\begin{array}{c}\text { Wave } \\
\text { number }\end{array}$ & Term combination \\
\hline $\begin{array}{c}25 \\
1 \\
20 \mathrm{w} \\
250 \\
1\end{array}$ & $\begin{array}{l}\text { A } \\
3062.043 \\
3062.914 \\
3064.22 \\
3064.834 \\
3065.882\end{array}$ & $\begin{array}{c}\mathrm{cm}^{-1} \\
32648.44 \\
32639.16 \\
32625.25 \\
32618.71 \\
32607.57\end{array}$ & $\begin{array}{l}c{ }^{3} \mathrm{~F}_{4}-63_{3}^{\circ} \\
a{ }^{1} \mathrm{G}_{4}-s^{3} \mathrm{D}_{3}^{\circ} \\
a^{3}{ }^{3} \mathrm{H}_{4}-52_{4}^{\circ} \\
b^{3} \mathrm{~F}_{4}-x^{3} \mathrm{G}_{5}^{\circ} \\
a^{5}{ }^{5} \mathrm{D}_{3}-x^{3} \mathrm{~F}_{2}^{\circ}\end{array}$ & $\begin{array}{r}80 \\
30 \\
100 \\
25 \\
50\end{array}$ & $\begin{array}{c}A \\
3118.687 \\
3120.542 \\
3124.164 \\
3124.362 \\
3124.601\end{array}$ & $\begin{array}{c}\mathrm{cm}^{-1} \\
32055.48 \\
32036.42 \\
31999.29 \\
31997.26 \\
31994.81\end{array}$ & $\begin{array}{l}a{ }^{3} \mathrm{P}_{1}-u^{3} \mathrm{D}_{1}^{\circ} \\
a^{3} \mathrm{G}_{5}-w^{5} \mathrm{D}_{4}^{\circ} \\
a^{3} \mathrm{~F}_{2}-x^{3} \mathrm{~F}_{2}^{\circ} \\
a \quad{ }^{5} \mathrm{P}_{3}-x^{3} \mathrm{D}_{3}^{\circ} \\
a^{1} \mathrm{G}_{4}-14_{4}^{\circ}\end{array}$ \\
\hline $\begin{array}{r}5 \\
50 \\
30 \\
80 \\
60\end{array}$ & $\begin{array}{l}3067.665 \\
3069.181 \\
3071.484 \\
3073.337 \\
3076.773\end{array}$ & $\begin{array}{l}32588.61 \\
32572.52 \\
32548.09 \\
32528.47 \\
32492.15\end{array}$ & $\begin{array}{l}a{ }^{3} \mathrm{D}_{3}-41_{2}^{\circ} \\
a{ }^{3} \mathrm{~F}_{2}-z{ }^{1} \mathrm{D}_{2}^{\circ} \\
a^{3} \mathrm{G}_{4}-y{ }^{1} \mathrm{G}_{4}^{\circ} \\
b{ }^{3} \mathrm{~F}_{2}-x^{3} \mathrm{G}_{3}^{\circ} \\
a^{3} \mathrm{~F}_{4}-z^{1} \mathrm{G}_{4}^{\circ}\end{array}$ & $\begin{array}{r}15 \\
100 \\
15 \\
2 \\
2\end{array}$ & $\begin{array}{l}3125.651 \\
3125.958 \\
3126.613 \\
3127.258 \\
3128.085\end{array}$ & $\begin{array}{l}31984.06 \\
31980.92 \\
31974.22 \\
31967.63 \\
31959.18\end{array}$ & $\begin{array}{l}a^{3} \mathrm{G}_{4}-x{ }^{5} \mathrm{~F}_{3}^{\circ} \\
a{ }^{5} \mathrm{~F}_{3}-z^{5} \mathrm{P}_{3}^{\circ} \\
a^{3} \mathrm{D}_{3}-32_{2}^{\circ} \\
a^{5} \mathrm{D}_{4}-y^{3} \mathrm{G}_{5}^{\circ} \\
a^{5} \mathrm{D}_{2}-x^{3} \mathrm{D}_{1}^{\circ}\end{array}$ \\
\hline $\begin{array}{r}20 \\
30 \\
1 \\
50 \\
75\end{array}$ & $\begin{array}{l}3077.057 \\
3077.542 \\
3078.679 \\
3080.188 \\
3080.898\end{array}$ & $\begin{array}{l}32489.15 \\
32484.03 \\
32472.03 \\
32456.12 \\
32448.64\end{array}$ & $\begin{array}{ll}a & { }^{5} \mathrm{P}_{3}-x^{3} \mathrm{~F}_{3}^{\circ} \\
a & { }^{3} \mathrm{P}_{2}-y^{3} \mathrm{~S}_{1}^{\circ} \\
a^{3} \mathrm{D}_{2}-22_{3}^{\circ} \\
b \quad{ }^{3} \mathrm{~F}_{2}-y{ }^{1} \mathrm{D}_{2}^{\circ} \\
a^{3} \mathrm{P}_{1}-x^{3} \mathrm{P}_{2}^{2}\end{array}$ & $\begin{array}{r}1 \\
2 \\
30 \\
75 \\
100\end{array}$ & $\begin{array}{l}3128.920 \\
3129.135 \\
3129.601 \\
3129.835 \\
3132.874\end{array}$ & $\begin{array}{l}31950.65 \\
31948.45 \\
31943.70 \\
31941.31 \\
31910.33\end{array}$ & $\begin{array}{ll}a & { }^{5} \mathrm{D}_{4}-y^{3} \mathrm{~F}_{8} \\
c & { }^{3} \mathrm{~F}_{2}-63_{3}^{\circ} \\
a & { }^{5} \mathrm{D}_{1}-x^{3} \mathrm{D}_{i}^{\circ} \\
a & { }^{5} \mathrm{~F}_{1}-z^{5} \mathrm{P}_{1}^{\circ} \\
a^{3}{ }^{3} \mathrm{P}_{2}-w^{3} \mathrm{D}_{2}^{\circ}\end{array}$ \\
\hline $\begin{array}{r}60 \\
30 \\
4 \\
5 \\
75\end{array}$ & $\begin{array}{l}3083.145 \\
3084.521 \\
3084.877 \\
3085.473 \\
3086.076\end{array}$ & $\begin{array}{l}32425.00 \\
32410.53 \\
32406.79 \\
32400.53 \\
32394.20\end{array}$ & 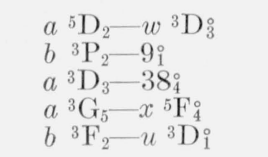 & $\begin{array}{r}4 \\
20 \\
1 \\
2\end{array}$ & $\begin{array}{l}3133.511 \\
3133.687 \\
3134.631 \\
3134.777\end{array}$ & $\begin{array}{l}31903.84 \\
31902.05 \\
31892.44 \\
31890.95\end{array}$ & $\begin{array}{l}a{ }^{3} \mathrm{H}_{5}-31_{4}^{\circ} \\
b{ }^{3} \mathrm{P}_{2}-7_{3}^{\circ} \\
a^{3} \mathrm{G}_{5}-y^{3} \mathrm{H}_{5}^{\circ} \\
a^{3} \mathrm{D}_{2}-w^{3} \mathrm{~F}_{3}^{\circ} \\
a^{5} \mathrm{D}_{2}-z^{1} \mathrm{~F}_{3}^{\circ}\end{array}$ \\
\hline $\begin{array}{l}20 \\
10 \\
30 \\
1 h \\
25\end{array}$ & $\begin{array}{l}3086.520 \\
3086.771 \\
3086.929 \\
3087.924 \\
3088.078\end{array}$ & $\begin{array}{l}32389.54 \\
32386.91 \\
32385.25 \\
32374.82 \\
32373.20\end{array}$ & $\begin{array}{lll}a & { }^{5} \mathrm{P}_{2}-y & { }^{3} \mathrm{~F}_{2}^{\circ} \\
a & { }^{5} \mathrm{P}_{1}-x & { }^{3} \mathrm{D}_{2}^{\circ} \\
a^{3} \mathrm{G}_{4}-x & { }^{1} \mathrm{~F}_{3}^{\circ} \\
a^{3} \mathrm{P}_{2}-y & { }^{1} \mathrm{~F}_{3}^{\circ} \\
a^{5}{ }^{5} \mathrm{D}_{3}-z & { }^{1} \mathrm{~F}_{3}^{\circ}\end{array}$ & $\begin{array}{r}2 \\
25 \\
5 \\
100 \\
30\end{array}$ & $\begin{array}{l}3135.066 \\
3135.933 \\
3136.349 \\
3136.554 \\
3136.911\end{array}$ & $\begin{array}{l}31888.01 \\
31879.20 \\
31874.97 \\
31872.89 \\
31869.26\end{array}$ & $\begin{array}{l}a{ }^{1} \mathrm{D}_{2}-43_{3}^{\circ} \\
b \\
{ }^{3} \mathrm{~F}_{3}-w^{3} \mathrm{D}_{2}^{\circ} \\
a \\
{ }^{3} \mathrm{H}_{6}-21_{5}^{\circ} \\
a \\
{ }^{5} \mathrm{P}_{2}-y^{3} \mathrm{P}_{1} \\
a^{3} \mathrm{H}_{5}-30_{4}^{\circ}\end{array}$ \\
\hline $\begin{array}{r}1 \\
80 \\
60 \\
50 \\
40\end{array}$ & $\begin{array}{l}3088.245 \\
3089.147 \\
3089.802 \\
3090.225 \\
3090.896\end{array}$ & $\begin{array}{l}32371.45 \\
32362.00 \\
32355.14 \\
32350.71 \\
32343.69\end{array}$ & 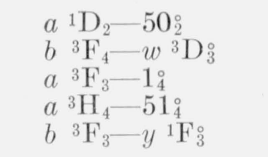 & $\begin{array}{r}10 \\
50 \\
10 \\
75 \\
125\end{array}$ & $\begin{array}{l}\text { 3138. } 761 \\
3139.273 \\
3140.086 \\
3140.485 \\
3140.979\end{array}$ & $\begin{array}{l}31850.48 \\
31845.28 \\
31837.04 \\
31832.99 \\
31827.99\end{array}$ & $\begin{array}{l}a{ }^{3} \mathrm{G}_{3}-7_{3}^{\circ} \\
a^{3} \mathrm{G}_{4}-y^{3} \mathrm{H}_{4}^{\circ} \\
a^{3} \mathrm{H}_{4}-43_{3}^{\circ} \\
a{ }^{3} \mathrm{~F}_{2}-x^{3} \mathrm{D}_{i}^{\circ} \\
b^{3} \mathrm{~F}_{4}-z^{1}{ }^{1} \mathrm{~F}_{3}\end{array}$ \\
\hline $\begin{array}{r}70 \\
100 \\
40 \\
75 \\
3\end{array}$ & $\begin{array}{l}3091.867 \\
3096.565 \\
3097.225 \\
3097.606 \\
3097.865\end{array}$ & $\begin{array}{l}32333.53 \\
32284.48 \\
32277.60 \\
32273.63 \\
32270.93\end{array}$ & $\begin{array}{lll}a & 5 \mathrm{~F}_{2}-z & { }^{5} \mathrm{P}_{1} \\
b & { }^{3} \mathrm{~F}_{3}-x^{3} \mathrm{G}_{4}^{\circ} \\
b & { }^{3} \mathrm{P}_{2}-t^{3} \mathrm{D}_{2}^{\circ} \\
a & { }^{3} \mathrm{P}_{2}-v^{3} \mathrm{D}_{2}^{\circ} \\
a^{3} \mathrm{P}_{2}-w^{3} \mathrm{D}_{1}^{\circ}\end{array}$ & $\begin{array}{r}5 \\
1 \\
150 \\
1 \\
1\end{array}$ & $\begin{array}{l}3142.522 \\
3142.88 \\
3144.265 \\
3144.502 \\
3144.579\end{array}$ & $\begin{array}{l}31812.36 \\
31808.74 \\
31794.72 \\
31792.33 \\
31791.55\end{array}$ & $\begin{array}{l}a^{3} \mathrm{D}_{3}-29_{3}^{\circ} \\
b^{3} \mathrm{P}_{1}-w^{5} \mathrm{D}_{2}^{\circ} \\
a^{1} \mathrm{G}_{4}-x^{1} \mathrm{H}_{5}^{\circ} \\
a^{3} \mathrm{P}_{2}-z^{1} \mathrm{P}_{1}^{\circ} \\
a^{3} \mathrm{G}_{5}-2_{\varnothing}^{\circ}\end{array}$ \\
\hline $\begin{array}{r}250 \\
10 \\
100 \\
10 \\
2\end{array}$ & $\begin{array}{l}3099.284 \\
3100.582 \\
3100.836 \\
3102.364 \\
3103.748\end{array}$ & $\begin{array}{l}32256.16 \\
32242.65 \\
32240.01 \\
32224.13 \\
32209.76\end{array}$ & $\begin{array}{lll}a & 5 \mathrm{~F}_{4}-y & { }^{5} \mathrm{D}_{4}^{\circ} \\
b & { }^{3} \mathrm{~F}_{3}-v^{3} \mathrm{D}_{2}^{\circ} \\
a & 5 \mathrm{~F}_{4}-y^{5} \mathrm{D}_{3}^{\circ} \\
a^{3} \mathrm{G}_{3}-t^{3} \mathrm{D}_{2}^{\circ} \\
b^{3}{ }^{3} \mathrm{P}_{1}-w^{5} \mathrm{D}_{1}^{\circ}\end{array}$ & $\begin{array}{r}1 \\
15 \\
50 \\
100 \\
10\end{array}$ & $\begin{array}{l}\text { 3144. } 640 \\
3144.715 \\
3146.075 \\
3147.214 \\
3148.021\end{array}$ & $\begin{array}{l}31790.93 \\
31790.18 \\
31776.44 \\
31764.94 \\
31756.79\end{array}$ & $\begin{array}{l}a{ }^{3} \mathrm{G}_{4}-x{ }^{5} \mathrm{~F}_{4}^{\circ} \\
a{ }^{5} \mathrm{D}_{4}-z^{3} \mathrm{H}_{4}^{\circ} \\
a{ }^{5} \mathrm{~F}_{1}-z^{5} \mathrm{P}_{2}^{\circ} \\
a^{3} \mathrm{~F}_{2}-z{ }^{1} \mathrm{~F}_{3}^{\circ} \\
a^{3} \mathrm{H}_{4}-42_{4}^{\circ}\end{array}$ \\
\hline $\begin{array}{l}50 \\
50 \\
50 \\
50\end{array}$ & $\begin{array}{l}3104.457 \\
3105.278 \\
3105.407 \\
3106.837\end{array}$ & $\begin{array}{l}32202.41 \\
32193.90 \\
32192.56 \\
32177.74\end{array}$ & $\begin{array}{l}a{ }^{5} \mathrm{D}_{2}-x^{3} \mathrm{~F}_{3}^{\circ} \\
a^{3} \mathrm{D}_{2}-w^{3} \mathrm{~F}_{2}^{\circ} \\
a^{5} \mathrm{D}_{3}-x^{3} \mathrm{D}_{3}^{\circ} \\
a^{3} \mathrm{~F}_{3}-y^{3} \mathrm{G}_{4}^{\circ} \\
a^{5} \mathrm{P}_{3}-z^{1} \mathrm{~F}_{3}^{\circ}\end{array}$ & $\begin{array}{r}75 \\
4 \\
100 \\
80 \\
2\end{array}$ & $\begin{array}{l}3148.493 \\
3150.172 \\
3150.699 \\
3151.363 \\
3151.686\end{array}$ & $\begin{array}{l}31752.03 \\
31735.11 \\
31729.80 \\
31723.12 \\
31719.86\end{array}$ & $\begin{array}{l}a{ }^{3} \mathrm{~F}_{4}-z^{3} \mathrm{H}_{5}^{\circ} \\
a^{3} \mathrm{D}_{2}-15_{1}^{\circ} \\
a{ }^{5} \mathrm{P}_{2}-y{ }^{5} \mathrm{P}_{1}^{\circ} \\
a{ }^{3} \mathrm{P}_{1}-u^{3} \mathrm{D}_{2}^{\circ} \\
a^{1} \mathrm{D}_{2}-40_{3}^{\circ}\end{array}$ \\
\hline $\begin{array}{r}150 \\
30 \\
35 \\
75 \\
50\end{array}$ & $\begin{array}{l}3107.720 \\
3108.423 \\
3109.394 \\
3110.536 \\
3111.910\end{array}$ & $\begin{array}{l}32168.60 \\
32161.32 \\
32151.28 \\
32139.48 \\
32125.29\end{array}$ & $\begin{array}{l}a{ }^{5} \mathrm{~F}_{2}-z{ }^{5} \mathrm{P}_{2}^{\circ} \\
a^{3} \mathrm{~F}_{4}-y{ }^{5} \mathrm{P}_{3}^{0} \\
a^{3} \mathrm{~F}_{3}-y{ }^{3} \mathrm{G}_{3}^{\circ} \\
b^{3} \mathrm{~F}_{4}-x^{3} \mathrm{~F}_{3}^{\circ} \\
a^{5} \mathrm{D}_{2}-x^{3} \mathrm{~F}_{2}^{\circ}\end{array}$ & $\begin{array}{c}1 \\
1 h \\
150 \\
25 \\
3\end{array}$ & $\begin{array}{l}3152.611 \\
3153.583 \\
3153.831 \\
3154.428 \\
3155.895\end{array}$ & $\begin{array}{l}31710.56 \\
31700.78 \\
31698.29 \\
31692.29 \\
31677.56\end{array}$ & $\begin{array}{lll}a & { }^{5} \mathrm{D}_{2}-x^{3} \mathrm{D}_{3}^{\circ} \\
b & { }^{3} \mathrm{P}_{0}-y & { }^{1} \mathrm{P}_{1}^{\circ} \\
a & { }^{5} \mathrm{P}_{2}-y & { }^{3} \mathrm{P}_{2}^{\circ} \\
b & { }^{3} \mathrm{~F}_{3}-x^{3} \mathrm{~F}_{4}^{\circ} \\
a^{3} \mathrm{D}_{3}-28_{3}^{\circ}\end{array}$ \\
\hline $\begin{array}{r}30 \\
70 \\
80 \\
8 \\
50\end{array}$ & $\begin{array}{l}3112.299 \\
3112.676 \\
3113.393 \\
3115.436 \\
3116.657\end{array}$ & $\begin{array}{l}32121.27 \\
32117.38 \\
32109.99 \\
32088.93 \\
32076.36\end{array}$ & $\begin{array}{l}b{ }^{3} \mathrm{P}_{1}-11_{0}^{\circ} \\
a^{3} \mathrm{P}_{1}-y{ }^{1} \mathrm{D}_{2}^{\circ} \\
a^{5} \mathrm{D}_{1}-x^{3} \mathrm{~F}_{2}^{\circ} \\
a^{3} \mathrm{P}_{0}-u^{3} \mathrm{D}_{1}^{\circ} \\
a^{3} \mathrm{~F}_{2}-x^{3} \mathrm{~F}_{3}^{\circ}\end{array}$ & $\begin{array}{l}25 \\
20 \\
25 \\
20\end{array}$ & $\begin{array}{l}3156.821 \\
3157.090 \\
3157.414 \\
3157.626\end{array}$ & $\begin{array}{l}31668.27 \\
31665.57 \\
31662.32 \\
31660.20\end{array}$ & $\begin{array}{l}a{ }^{5} \mathrm{P}_{3}-1{ }_{4}^{\circ} \\
a^{3} \mathrm{G}_{3}-y{ }^{1} \mathrm{G}_{4}^{\circ} \\
a{ }^{5} \mathrm{P}_{3}-y{ }^{3} \mathrm{~F}_{2}^{\circ} \\
b{ }^{3} \mathrm{~F}_{2}-y{ }^{3} \mathrm{~S}_{1}^{\circ} \\
a^{5}{ }^{5} \mathrm{D}_{3}-y{ }^{3} \mathrm{G}_{3}^{\circ}\end{array}$ \\
\hline $\begin{array}{r}25 \\
3 \\
4 \\
80\end{array}$ & $\begin{array}{l}3116.834 \\
3117.454 \\
3117.631 \\
3118.065\end{array}$ & $\begin{array}{l}32074.54 \\
32068.16 \\
32066.34 \\
32061.88\end{array}$ & $\begin{array}{l}b \quad{ }^{3} \mathrm{P}_{1}-9_{1}^{\circ} \\
c{ }^{3} \mathrm{~F}_{4}-62_{4}^{\circ} \\
a^{3} \mathrm{H}_{4}-47_{4}^{\circ} \\
a^{3} \mathrm{D}_{1}-41_{2}^{\circ} \\
b^{3}{ }^{3} \mathrm{~F}_{2}-u^{3} \mathrm{D}_{2}^{\circ}\end{array}$ & $\begin{array}{r}15 \\
1 \\
125 \\
200 \\
1\end{array}$ & $\begin{array}{l}3157.850 \\
3158.057 \\
3158.899 \\
3159.935 \\
3160.924\end{array}$ & $\begin{array}{l}31657.95 \\
31655.88 \\
31647.44 \\
31637.06 \\
31627.16\end{array}$ & $\begin{array}{l}a{ }^{3} \mathrm{~F}_{3}-y^{3} \mathrm{P}_{2}^{\circ} \\
a{ }^{3} \mathrm{G}_{5}-w^{3} \mathrm{G}_{4}^{\circ} \\
b \quad{ }^{3} \mathrm{~F}_{4}-x^{3} \mathrm{D}_{3}^{\circ} \\
a \quad \mathrm{~F}_{3}-y^{5} \mathrm{D}_{2}^{\circ} \\
a^{3}{ }^{3} \mathrm{D}_{3}-27_{4}^{\circ}\end{array}$ \\
\hline
\end{tabular}


TABLE 3-Continued

\begin{tabular}{|c|c|c|c|c|c|c|c|}
\hline Intensity & $\begin{array}{l}\text { Wave- } \\
\text { length }\end{array}$ & $\begin{array}{c}\text { Wave } \\
\text { number }\end{array}$ & Term combination & Intensity & $\begin{array}{l}\text { Wave- } \\
\text { length }\end{array}$ & $\begin{array}{c}\text { Wave } \\
\text { number }\end{array}$ & Term combination \\
\hline $\begin{array}{l}3 \\
1 h \\
25 \\
25 \\
1\end{array}$ & $\begin{array}{c}A \\
3162.223 \\
3162.589 \\
3163.831 \\
3165.195 \\
3165.887\end{array}$ & $\begin{array}{c}c m^{-1} \\
31614.17 \\
31610.52 \\
31598.11 \\
31584.49 \\
31577.59\end{array}$ & $\begin{array}{l}a^{3}{ }^{3} \mathrm{D}_{1}-33_{2}^{\circ} \\
b^{3} \mathrm{P}_{1}-x^{5} \mathrm{~F}_{2}^{\circ} \\
a^{3} \mathrm{D}_{3}-25_{3}^{\circ} \\
a^{3} \mathrm{~F}_{2}-x^{3} \mathrm{D}_{3}^{\circ} \\
a^{3} \mathrm{H}_{5}-27_{4}^{\circ}\end{array}$ & $\begin{array}{r}3 \\
30 \\
3 \\
100 \\
50\end{array}$ & $\begin{array}{c}A \\
3209.489 \\
3210.169 \\
3211.144 \\
3212.983 \\
3213.355\end{array}$ & $\begin{array}{c}\mathrm{cm}^{-1} \\
31148.61 \\
31142.01 \\
31132.56 \\
31114.74 \\
31111.13\end{array}$ & $\begin{array}{ll}a^{3} \mathrm{D}_{3}-s^{3} \mathrm{D}_{3}^{\circ} \\
a & { }^{3} \mathrm{P}_{0}-w^{3} \mathrm{D}_{1}^{\circ} \\
a & { }^{3} \mathrm{P}_{2}-z^{1} \mathrm{D}_{2}^{\circ} \\
b & { }^{3} \mathrm{~F}_{4}-y^{3} \mathrm{G}_{3}^{\circ} \\
a^{3} \mathrm{P}_{1}-v^{3} \mathrm{D}_{2}^{\circ}\end{array}$ \\
\hline $\begin{array}{r}2 \\
5 \\
8 \\
40 \\
30\end{array}$ & $\begin{array}{l}3166.339 \\
3167.381 \\
3167.820 \\
3168.243 \\
3168.489\end{array}$ & $\begin{array}{l}31573.08 \\
31562.69 \\
31558.32 \\
31554.10 \\
31551.65\end{array}$ & $\begin{array}{l}a{ }^{1} \mathrm{G}_{4}-12_{4}^{\circ} \\
a{ }^{5} \mathrm{P}_{1}-x^{3} \mathrm{~F}_{2}^{\circ} \\
a{ }^{1} \mathrm{D}_{2}-39_{1}^{\circ} \\
a{ }^{5} \mathrm{D}_{4}-z^{1} \mathrm{G}_{4}^{\circ} \\
b^{3} \mathrm{P}_{2}-y{ }^{5} \mathrm{~S}_{2}^{\circ}\end{array}$ & $\begin{array}{r}50 \\
50 \\
100 \\
5\end{array}$ & $\begin{array}{l}3214.335 \\
3215.910 \\
3216.528 \\
3217.523\end{array}$ & $\begin{array}{l}31101.65 \\
31086.42 \\
31080.45 \\
31070.84\end{array}$ & $\begin{array}{l}b{ }^{3} \mathrm{~F}_{3}-z^{1} \mathrm{D}_{2}^{\circ} \\
a^{3} \mathrm{G}_{3}-x^{5} \mathrm{~F}_{3}^{\circ} \\
b^{3} \mathrm{~F}_{2}-w^{3} \mathrm{D}_{2}^{\circ} \\
a^{5} \mathbf{F}_{3}-z^{3} \mathrm{~F}_{2}^{\circ} \\
a^{3} \mathrm{D}_{3}-19_{4}^{\circ}\end{array}$ \\
\hline $\begin{array}{l}60 \\
80 \\
50 \\
25 \\
25\end{array}$ & $\begin{array}{l}3168.560 \\
3170.088 \\
3171.239 \\
3173.110 \\
3173.394\end{array}$ & $\begin{array}{l}31550.95 \\
31535.74 \\
31524.30 \\
31505.71 \\
31502.89\end{array}$ & $\begin{array}{l}b{ }^{3} \mathrm{~F}_{2}-y{ }^{1} \mathrm{~F}_{3}^{\circ} \\
a^{3} \mathrm{G}_{5}-w^{3} \mathrm{G}_{5}^{\circ} \\
a^{5} \mathrm{D}_{0}-x^{3} \mathrm{D}_{1}^{\circ} \\
a^{5} \mathrm{P}_{3}-y^{3} \mathrm{G}_{4}^{\circ} \\
a^{3} \mathrm{G}_{3}-x^{1} \mathrm{~F}_{3}^{\circ}\end{array}$ & $\begin{array}{r}1 \\
8 \\
50 \\
200 \\
25\end{array}$ & $\begin{array}{l}3218.920 \\
3219.148 \\
3220.081 \\
3223.279 \\
3224.658\end{array}$ & $\begin{array}{l}31057.35 \\
31055.15 \\
31046.15 \\
31015.35 \\
31002.09\end{array}$ & $\begin{array}{l}a^{3} \mathrm{D}_{3}-w^{3} \mathrm{~F}_{2}^{\circ} \\
a^{1} \mathrm{G}_{4}-w^{3} \mathrm{~F}_{4}^{\circ} \\
a^{3} \mathrm{G}_{4}-w^{3} \mathrm{G}_{4}^{\circ} \\
a^{5} \mathrm{~F}_{2}-y^{5} \mathrm{D}_{2}^{\circ} \\
a^{3} \mathrm{D}_{2}-9_{1}^{\circ}\end{array}$ \\
\hline $\begin{array}{r}60 \\
50 \\
25 \\
2 \\
30\end{array}$ & $\begin{array}{l}3174.128 \\
3176.286 \\
3178.736 \\
3179.025 \\
3179.263\end{array}$ & $\begin{array}{l}31495.60 \\
31474.21 \\
31449.95 \\
31447.09 \\
31444.73\end{array}$ & $\begin{array}{l}b{ }^{3} \mathrm{~F}_{4}-z{ }^{1} \mathrm{H}_{5}^{\circ} \\
a^{3} \mathrm{D}_{2}-y{ }^{1} \mathrm{P}_{1}^{\circ} \\
b{ }^{3} \mathrm{~F}_{2}-v{ }^{3} \mathrm{D}_{2}^{\circ} \\
a^{1} \mathrm{D}_{2}-35_{3}^{\circ} \\
a^{3} \mathrm{D}_{3}-23_{3}^{\circ}\end{array}$ & $\begin{array}{r}100 \\
75 \\
40 \\
150 \\
2\end{array}$ & $\begin{array}{l}3226.380 \\
3227.889 \\
3228.158 \\
3228.531 \\
3228.724\end{array}$ & $\begin{array}{l}30985.54 \\
30971.06 \\
30968.48 \\
30964.90 \\
30963.05\end{array}$ & $\begin{array}{lll}a & 5 \mathbf{F}_{1}-y & { }^{5} \mathrm{D}_{1}^{\circ} \\
a & { }^{5} \mathbf{P}_{3}-y & { }^{3} \mathbf{P}_{2}^{\circ} \\
b & { }^{3} \mathbf{F}_{2}-z & { }^{1} \mathbf{P}_{1}^{\circ} \\
a & { }^{5} \mathbf{P}_{2}-y & { }^{5} \mathbf{P}_{2}^{\circ} \\
a^{3} \mathbf{G}_{3}-y & { }^{3} \mathbf{H}_{4}^{\circ}\end{array}$ \\
\hline $\begin{array}{l}5 \\
1 \\
2 \\
1\end{array}$ & $\begin{array}{l}3181.189 \\
3183.137 \\
3183.513 \\
3184.177\end{array}$ & $\begin{array}{l}31425.70 \\
31406.47 \\
31402.76 \\
31396.21\end{array}$ & $\begin{array}{l}b{ }^{3} \mathrm{P}_{2}-w^{5} \mathrm{D}_{3}^{\circ} \\
a^{3} \mathrm{H}_{4}-36_{5}^{\circ} \\
a^{3} \mathrm{H}_{5}-24_{5}^{\circ} \\
a^{5} \mathrm{P}_{1}-x^{3} \mathrm{D}_{1}^{\circ} \\
a^{3} \mathrm{H}_{4}-35_{3}^{\circ}\end{array}$ & $\begin{array}{r}50 \\
30 \\
80 \\
5 \\
10\end{array}$ & $\begin{array}{l}3229.768 \\
3230.621 \\
3232.753 \\
3233.519 \\
3234.428\end{array}$ & $\begin{array}{l}30953.04 \\
30944.87 \\
30924.46 \\
30917.14 \\
30908.45\end{array}$ & $\begin{array}{l}a{ }^{3} \mathrm{~F}_{3}-z^{1} \mathrm{G}_{4}^{\circ} \\
a^{3} \mathrm{H}_{6}-x{ }^{1} \mathrm{H}_{5}^{\circ} \\
a^{3} \mathrm{~F}_{3}-y{ }^{5} \mathrm{P}_{2}^{\circ} \\
a^{3} \mathrm{H}_{5}-17_{4}^{\circ} \\
a^{3} \mathrm{G}_{3}-x^{5} \mathrm{~F}_{4}^{\circ}\end{array}$ \\
\hline $\begin{array}{r}50 \\
10 \\
200 \\
1 \\
1\end{array}$ & $\begin{array}{l}3184.847 \\
3185.447 \\
3186.042 \\
3186.584 \\
3187.822\end{array}$ & $\begin{array}{l}31389.60 \\
31383.69 \\
31377.83 \\
31372.49 \\
31360.31\end{array}$ & $\begin{array}{l}a^{3} \mathrm{G}_{5}-y{ }^{1} \mathrm{H}_{5}^{\circ} \\
a^{3} \mathrm{P}_{2}-x^{3} \mathrm{D}_{2}^{\circ} \\
a{ }^{5} \mathrm{~F}_{2}-y{ }^{5} \mathrm{D}_{1}^{\circ} \\
a^{3} \mathrm{G}_{3}-w^{5} \mathrm{D}_{3}^{\circ} \\
a^{5} \mathrm{D}_{1}-y^{3} \mathrm{~F}_{2}^{\circ}\end{array}$ & $\begin{array}{r}100 \\
30 \\
30 \\
10\end{array}$ & $\begin{array}{l}3238.537 \\
3238.780 \\
3239.596 \\
3239.686\end{array}$ & $\begin{array}{l}\text { 30869. } 23 \\
30866.92 \\
30859.14 \\
30858.28\end{array}$ & $\begin{array}{l}a^{3} \mathrm{D}_{2}-t^{3} \mathrm{D}_{2}^{\circ} \\
a \mathrm{~F}_{2}-z^{3} \mathrm{D}_{1}^{\circ} \\
a^{3} \mathrm{P}_{2}-w^{3} \mathrm{D}^{\circ} \\
a^{5} \mathrm{D}_{2}-y^{3} \mathrm{P}_{1}^{\circ} \\
a^{5} \mathrm{D}_{3}-y^{3} \mathrm{~F}_{3}^{\circ}\end{array}$ \\
\hline $\begin{array}{r}40 \\
200 \\
25 \\
30\end{array}$ & $\begin{array}{l}3187.939 \\
3188.340 \\
3188.609 \\
3188.916\end{array}$ & $\begin{array}{l}31359.16 \\
31355.22 \\
31352.57 \\
31349.56\end{array}$ & $\begin{array}{l}a{ }^{5} \mathrm{~F}_{2}-z^{5} \mathrm{P}_{3}^{\circ} \\
a^{1} \mathrm{D}_{2}-34_{3}^{\circ} \\
a^{5} \mathrm{~F}_{3}-y^{5} \mathrm{D}_{4}^{\circ} \\
b^{3}{ }^{3} \mathrm{~F}_{3}-x^{3} \mathrm{D}_{2}^{\circ} \\
a^{3} \mathbf{F}_{3}-y^{3} \mathbf{F}_{3}^{\circ}\end{array}$ & $\begin{array}{c}1 \\
100 \\
1 h \\
40 \\
25\end{array}$ & $\begin{array}{l}3239.928 \\
3241.236 \\
3241.786 \\
3242.159 \\
3242.850\end{array}$ & $\begin{array}{l}30855.98 \\
30843.53 \\
30838.29 \\
30834.75 \\
30828.18\end{array}$ & $\begin{array}{l}a^{3} \mathrm{D}_{3}-16_{3}^{\circ} \\
a^{5} \mathrm{D}_{1}-y^{3} \mathrm{P}_{1} \\
b^{1} \mathrm{D}_{1}-63_{3}^{\circ} \\
a^{3} \mathrm{P}_{1}-z{ }^{1} \mathrm{~S}_{0}^{\circ} \\
a^{1} \mathrm{G}_{4}-x{ }^{1} \mathrm{G}_{4}^{\circ} \\
b^{3} \mathrm{~F}_{3}-w^{3} \mathrm{D}_{3}^{\circ}\end{array}$ \\
\hline $\begin{array}{r}30 \\
20 \\
200 \\
20 \\
100\end{array}$ & $\begin{array}{l}3189.297 \\
3189.726 \\
3189.979 \\
3191.778 \\
3192.068\end{array}$ & $\begin{array}{l}31345.81 \\
31341.60 \\
31339.11 \\
31321.45 \\
31318.60\end{array}$ & $\begin{array}{ll}a & 5 \mathrm{~F}_{5}-z^{5} \mathrm{G}_{4}^{\circ} \\
a^{3} \mathrm{G}_{5}-y^{3} \mathrm{H}_{6}^{\circ} \\
a \\
a^{5} \mathrm{~F}_{3}-y{ }^{5} \mathrm{D}_{3}^{\circ} \\
a^{3} \mathrm{P}_{1}-y^{3} \mathrm{~S}_{1}^{\circ} \\
b^{3} \mathrm{~F}_{4}-1_{4}^{\circ}\end{array}$ & $\begin{array}{r}100 \\
10 \\
15 \\
2 \\
15\end{array}$ & $\begin{array}{l}3243.506 \\
3244.334 \\
3244.456 \\
3246.257 \\
3248.841\end{array}$ & $\begin{array}{l}30821.94 \\
30814.08 \\
30812.92 \\
30795.82 \\
30771.33\end{array}$ & $\begin{array}{l}a^{3} \mathrm{~F}_{4}-x^{5} \mathrm{D}_{3}^{\circ} \\
a^{5} \mathrm{D}_{4}-z^{3} \mathrm{H}_{5}^{\circ} \\
a{ }^{5} \mathrm{P}_{1}-y^{3} \mathrm{~F}_{2}^{\circ} \\
b^{3} \mathrm{P}_{2}-4_{3}^{\circ} \\
a^{3} \mathrm{G}_{5}-z^{3} \mathrm{I}_{5}^{\circ} \\
a^{3} \mathrm{H}^{3}-28^{\circ}\end{array}$ \\
\hline $\begin{array}{r}40 \\
100 \\
15 \\
1 \\
200\end{array}$ & $\begin{array}{l}3193.509 \\
3194.746 \\
3195.317 \\
3195.909 \\
3196.606\end{array}$ & $\begin{array}{l}31304.47 \\
31292.35 \\
31286.75 \\
31280.96 \\
31274.14\end{array}$ & $\begin{array}{l}a{ }^{1} \mathrm{H}_{5}-61_{4}^{\circ} \\
a^{3} \mathrm{G}_{4}-y^{3} \mathrm{H}_{5}^{\circ} \\
b^{3} \mathrm{H}_{4}-63_{3}^{\circ} \\
a^{1}{ }^{1} \mathrm{D}_{2}-33_{2}^{\circ} \\
a^{5} \mathrm{~F}_{1}-y^{5} \mathrm{D}_{0}^{\circ}\end{array}$ & $\begin{array}{r}50 \\
5 \\
50 \\
10 \\
10\end{array}$ & $\begin{array}{l}3250.002 \\
3250.458 \\
3251.327 \\
3251.893 \\
3252.540\end{array}$ & $\begin{array}{l}30760.34 \\
30756.02 \\
30747.80 \\
30742.45 \\
30736.34\end{array}$ & $\begin{array}{l}a^{5} \mathrm{D}_{4}-y^{3} \mathrm{~F}_{4}^{\circ} \\
a^{3} \mathrm{D}_{3}-w^{3} \mathrm{~F}_{3}^{\circ} \\
a^{3} \mathrm{P}_{1}-w^{3} \mathrm{D}_{2}^{\circ} \\
a^{3} \mathrm{G}_{3}-4_{3}^{\circ} \\
a^{3} \mathrm{D}_{2}-w^{5} \mathrm{D}_{2}^{\circ}\end{array}$ \\
\hline $\begin{array}{r}40 \\
15 \\
1 \\
70 \\
3\end{array}$ & $\begin{array}{l}3198.329 \\
3199.119 \\
3199.528 \\
3201.512 \\
3201.812\end{array}$ & $\begin{array}{l}31257.29 \\
21249.58 \\
31245.58 \\
31226.22 \\
31223.29\end{array}$ & $\begin{array}{ll}a & { }^{3} \mathrm{P}_{2}-v^{3} \mathrm{D}_{3}^{\circ} \\
a & { }^{3} \mathbf{F}_{2}-y \\
b & { }^{3} \mathbf{F}_{2}^{\circ} \\
b & { }^{3} \mathrm{P}_{2}-5_{2}^{\circ} \\
b & { }^{3} \mathbf{F}_{3}-v^{3} \mathrm{D}_{3}^{\circ} \\
a^{5} \mathrm{D}_{4}-y & { }^{5} \mathbf{P}_{3}^{\circ}\end{array}$ & $\begin{array}{l}10 \\
15 \\
80 \\
80 \\
75\end{array}$ & $\begin{array}{l}3252.898 \\
3252.993 \\
3254.539 \\
3254.707 \\
3256.334\end{array}$ & $\begin{array}{l}30732.96 \\
30732.06 \\
30717.46 \\
30715.87 \\
30700.53\end{array}$ & $\begin{array}{l}a{ }^{3} \mathrm{~F}_{2}-y{ }^{3} \mathrm{P}_{1}^{\circ} \\
a^{3} \mathrm{G}_{5}-x{ }^{3} \mathrm{G}_{4}^{\circ} \\
a^{5} \mathrm{~F}_{2}-y{ }^{5} \mathrm{D}_{3}^{\circ} \\
a^{5} \mathrm{D}_{2}-y{ }^{5} \mathrm{P}_{1}^{\circ} \\
a^{5} \mathrm{D}_{1}-y{ }^{5} \mathrm{P}_{1}^{\circ}\end{array}$ \\
\hline $\begin{array}{r}40 \\
75 \\
50 \\
2 \\
2\end{array}$ & $\begin{array}{l}3202.598 \\
3204.071 \\
3205.318 \\
3205.736 \\
3206.491\end{array}$ & $\begin{array}{l}31215.63 \\
31201.28 \\
31189.14 \\
31185.07 \\
31177.73\end{array}$ & $\begin{array}{l}b{ }^{3} \mathrm{P}_{1}-y{ }^{5} \mathrm{~S}_{2}^{\circ} \\
a{ }^{5} \mathrm{~F}_{4}-z{ }^{3} \mathrm{~F}_{3}^{\circ} \\
a^{3} \mathrm{~F}_{3}-z^{3} \mathrm{H}_{4}^{\circ} \\
a^{3} \mathrm{H}_{5}-21_{5}^{\circ} \\
a^{5} \mathrm{D}_{2}-y^{3} \mathrm{G}_{3}^{\circ}\end{array}$ & $\begin{array}{r}5 \\
75 \\
100 \\
10 \\
400\end{array}$ & $\begin{array}{l}3256.611 \\
3258.042 \\
3259.677 \\
3260.167 \\
3260.367\end{array}$ & $\begin{array}{l}30697.92 \\
30684.43 \\
30669.04 \\
30664.43 \\
30662.55\end{array}$ & $\begin{array}{l}a^{5} \mathrm{D}_{3}-z^{3} \mathrm{H}_{4}^{\circ} \\
a^{5} \mathrm{D}_{2}-y{ }^{3} \mathrm{P}_{2}^{\circ} \\
a^{5} \mathrm{D}_{1}-y{ }^{3} \mathrm{P}_{2}^{\circ} \\
a^{1} \mathrm{G}_{4}-y{ }^{1} \mathrm{G}_{4}^{\circ} \\
a^{5} \mathrm{~F}_{4}-z^{3} \mathrm{G}_{3}^{3}\end{array}$ \\
\hline $\begin{array}{r}6 \\
25 \\
6 \\
8 \\
1\end{array}$ & $\begin{array}{l}3207.635 \\
3208.419 \\
3208.732 \\
3208.813 \\
3209.303\end{array}$ & $\begin{array}{l}31166.61 \\
31159.00 \\
31155.96 \\
31155.17 \\
31150.41\end{array}$ & $\begin{array}{ll}a & { }^{5} \mathrm{D}_{3}-y \\
a^{3} \mathrm{P}_{2}^{\circ} \\
{ }^{3} \mathrm{G}_{4}-x^{3} \mathrm{G}_{3}^{\circ} \\
b & { }^{3} \mathrm{~F}_{4}-y^{3} \mathrm{G}_{4} \\
b & { }^{3} \mathrm{P}_{2}-x^{5} \mathrm{~F}_{3}^{\circ} \\
b & { }^{3} \mathrm{P}_{0}-8_{1}\end{array}$ & $\begin{array}{r}60 \\
5 \\
100 \\
100 \\
100\end{array}$ & $\begin{array}{l}3261.128 \\
3263.131 \\
3263.856 \\
3264.561 \\
3264.663\end{array}$ & $\begin{array}{l}30655.40 \\
30636.58 \\
30629.78 \\
30623.16 \\
30622.21\end{array}$ & 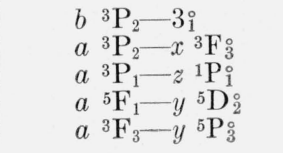 \\
\hline
\end{tabular}


Table 3-Continued

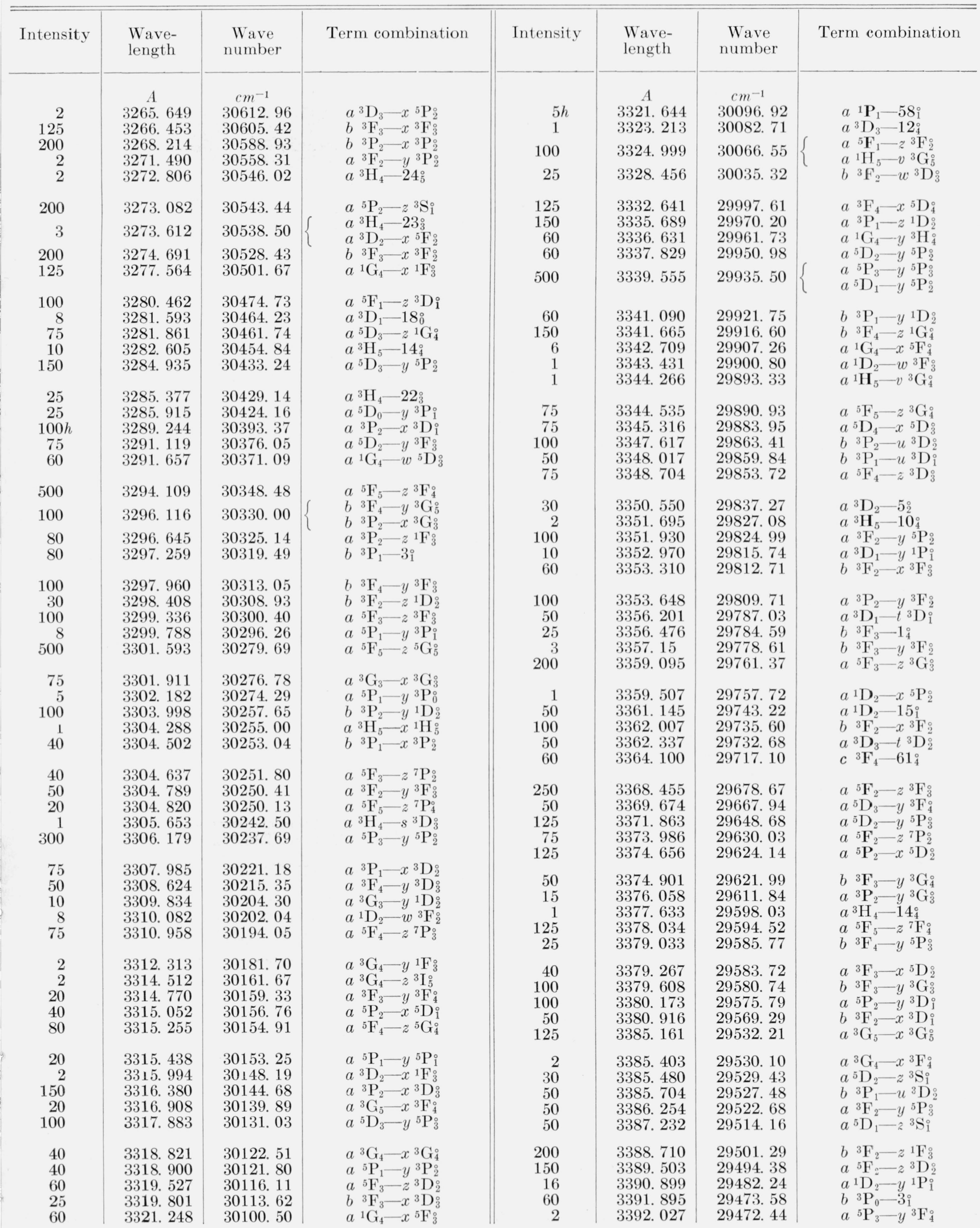


TABLE 3-Continued

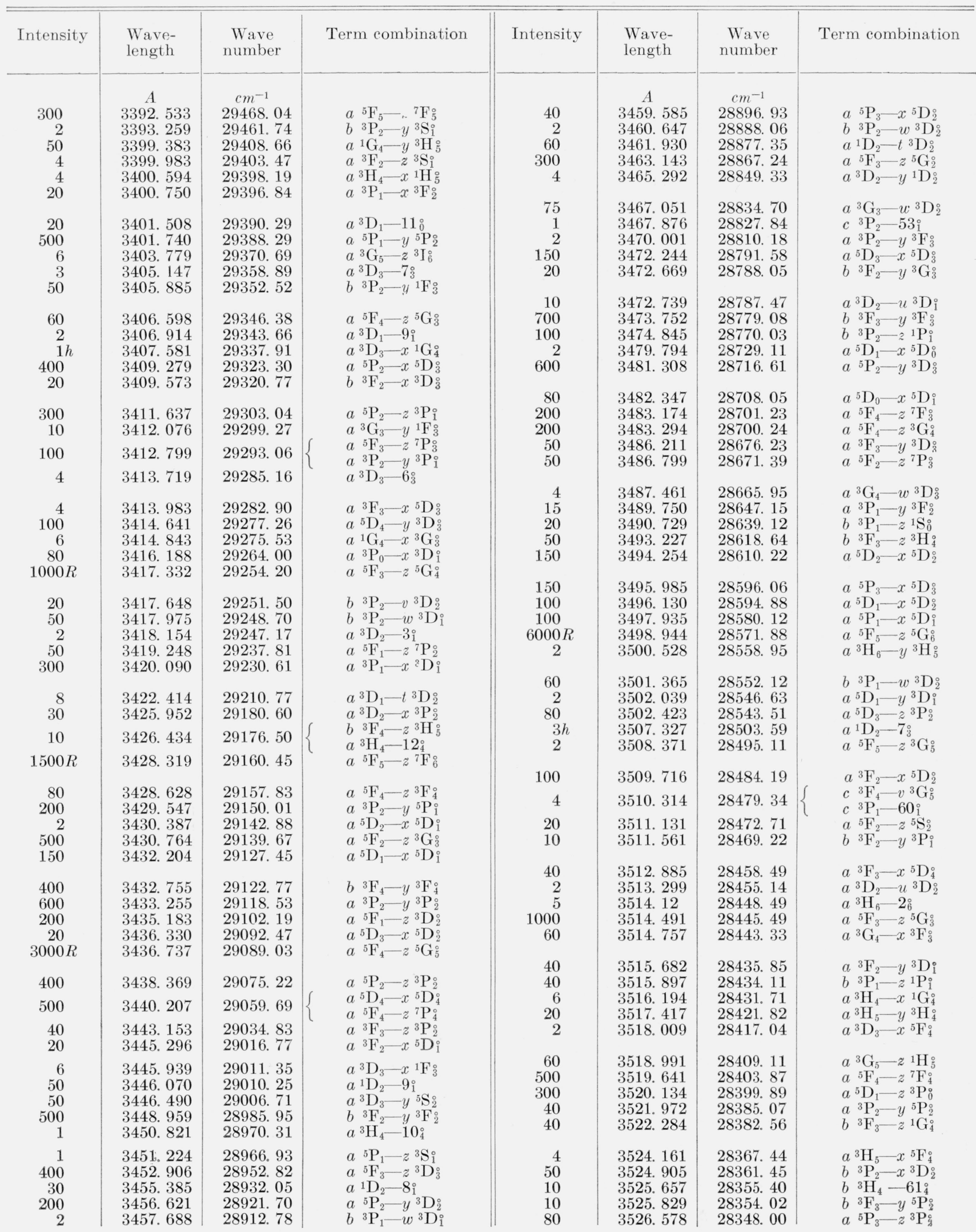


Table 3-Continued

\begin{tabular}{|c|c|c|c|c|c|c|c|}
\hline Intensity & $\begin{array}{l}\text { Wave- } \\
\text { length }\end{array}$ & $\begin{array}{c}\text { Wave } \\
\text { number }\end{array}$ & Term combination & Intensity & $\begin{array}{l}\text { Wave- } \\
\text { length }\end{array}$ & $\begin{array}{c}\text { Wave } \\
\text { number }\end{array}$ & Term combination \\
\hline $\begin{array}{r}250 \\
50 \\
2 \\
200 \\
10\end{array}$ & $\begin{array}{c}\text { A } \\
\text { 3528. } 679 \\
3529.292 \\
3529.621 \\
3531.389 \\
3531.808\end{array}$ & $\begin{array}{c}c m^{-1} \\
28331.12 \\
28326.20 \\
28323.56 \\
28309.38 \\
28306.02\end{array}$ & 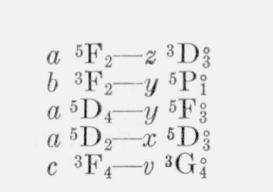 & $\begin{array}{r}40 \\
70 \\
30 \\
100 \\
150\end{array}$ & $\begin{array}{c}\text { A } \\
3575.054 \\
3577.540 \\
3578.686 \\
3579.773 \\
3584.198\end{array}$ & $\begin{array}{c}\mathrm{cm}^{-1} \\
27963.62 \\
27944.19 \\
27935.24 \\
27926.76 \\
27892.28\end{array}$ & $\begin{array}{l}a{ }^{3} \mathrm{P}_{2}-z{ }^{3} \mathrm{~S}_{1} \\
a^{3} \mathrm{D}_{2}-y{ }^{1} \mathrm{~F}_{3}^{\circ} \\
a^{3} \mathrm{~F}_{2}-z{ }^{3} \mathrm{P}_{2}^{\circ} \\
a^{5} \mathrm{~F}_{3}-z{ }^{7} \mathrm{~F}_{2}^{\circ} \\
a^{5} \mathrm{D}_{1}-y^{3} \mathrm{D}_{2}^{\circ}\end{array}$ \\
\hline $\begin{array}{r}300 \\
25 \\
50 \\
60 \\
200\end{array}$ & $\begin{array}{l}3532.810 \\
3533.917 \\
3535.059 \\
3535.32 \\
3535.382\end{array}$ & $\begin{array}{l}28297.99 \\
28289.13 \\
28279.99 \\
28277.90 \\
28277.41\end{array}$ & $\begin{array}{l}a{ }^{1} \mathrm{G}_{4}-y{ }^{1} \mathrm{~F}_{3}^{\circ} \\
a{ }^{5} \mathrm{D}_{2}-z^{3} \mathrm{P}_{1} \\
b^{3} \mathrm{P}_{0}-y^{3} \mathrm{~S}_{1}^{\circ} \\
a^{1}{ }^{1} \mathrm{G}_{4}-z^{3} \mathrm{I}_{5}^{\circ} \\
a^{5} \mathrm{~F}_{4}-z^{7} \mathrm{~F}_{5}^{\circ}\end{array}$ & $\begin{array}{c}30 \\
300 \\
2000 R \\
10 \\
60\end{array}$ & $\begin{array}{l}3585.812 \\
3587.204 \\
3589.220 \\
3590.525 \\
3590.884\end{array}$ & $\begin{array}{l}27879.73 \\
27868.91 \\
27853.26 \\
27843.13 \\
27840.35\end{array}$ & $\begin{array}{l}a{ }^{3} \mathrm{~F}_{3}-y{ }^{5} \mathrm{~F}_{2}^{\circ} \\
a^{3} \mathrm{H}_{5}-y{ }^{3} \mathrm{H}_{5}^{\circ} \\
a{ }^{5} \mathrm{~F}_{1}-z{ }^{5} \mathrm{G}_{2}^{\circ} \\
a^{3} \mathrm{D}_{2}-v^{3} \mathrm{D}_{2}^{\circ} \\
a^{3} \mathrm{D}_{2}-w^{3} \mathrm{D}_{1}^{\circ}\end{array}$ \\
\hline $\begin{array}{r}200 \\
60 \\
400 \\
200 \\
400\end{array}$ & $\begin{array}{l}3535.836 \\
3536.572 \\
3537.941 \\
3539.254 \\
3539.368\end{array}$ & $\begin{array}{l}28273.77 \\
28267.89 \\
28256.95 \\
28246.47 \\
28245.56\end{array}$ & 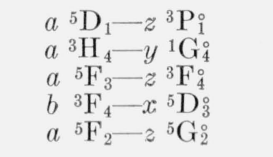 & $\begin{array}{c}2 \\
3000 R \\
50 \\
2000 R \\
10\end{array}$ & $\begin{array}{l}3591.333 \\
3593.029 \\
3596.057 \\
3596.185 \\
3598.225\end{array}$ & $\begin{array}{l}27836.87 \\
27823.73 \\
27800.30 \\
27799.32 \\
27783.55\end{array}$ & $\begin{array}{ll}b & { }^{3} \mathrm{P}_{2}-w^{3} \mathrm{D}_{3}^{\circ} \\
a & { }^{5} \mathrm{~F}_{2}-z^{5} \mathrm{G}_{3}^{\circ} \\
a & { }^{5} \mathrm{~F}_{3}-z^{7} \mathrm{~F}_{3}^{\circ} \\
a & { }^{5} \mathrm{~F}_{3}-z^{3} \mathrm{G}_{4}^{\circ} \\
a^{3} \mathrm{G}_{3}-w^{3} \mathrm{D}_{3}^{\circ}\end{array}$ \\
\hline $\begin{array}{r}50 \\
2 \\
50 \\
200 \\
2\end{array}$ & $\begin{array}{l}3540.210 \\
3540.673 \\
3541.040 \\
3541.622 \\
3545.713\end{array}$ & $\begin{array}{l}28238.84 \\
28235.15 \\
28232.22 \\
28227.58 \\
28195.02\end{array}$ & 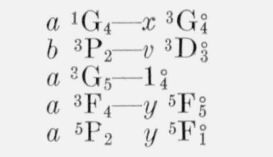 & $\begin{array}{r}20 \\
30 \\
1000 \\
150 \\
2\end{array}$ & $\begin{array}{l}3598.478 \\
3599.400 \\
3599.769 \\
3601.487 \\
3601.955\end{array}$ & $\begin{array}{l}27781.60 \\
27774.48 \\
27771.64 \\
27758.39 \\
27754.78\end{array}$ & $\begin{array}{lll}a & { }^{3} \mathrm{~F}_{2}-y & { }^{3} \mathrm{D}_{2}^{\circ} \\
b & { }^{3} \mathrm{P}_{1}-z & { }^{1} \mathrm{D}_{2}^{\circ} \\
a & { }^{5} \mathrm{P}_{3}-x & { }^{5} \mathrm{D}_{4}^{\circ} \\
a^{3} \mathrm{H}_{5}-2_{6}^{\circ} \\
a^{1}{ }^{1} \mathrm{D}_{2}-x^{5} \mathrm{~F}_{3}^{\circ}\end{array}$ \\
\hline $\begin{array}{r}10 \\
4 \\
100 \\
50 \\
50\end{array}$ & $\begin{array}{l}3545.797 \\
3546.014 \\
3546.982 \\
3547.184 \\
3547.380\end{array}$ & $\begin{array}{l}28194.35 \\
28192.62 \\
28184.93 \\
28183.33 \\
28181.77\end{array}$ & $\begin{array}{ll}a & { }^{5} \mathrm{P}_{3}-y^{3} \mathrm{D}_{2}^{\circ} \\
a & { }^{3} \mathrm{H}_{6}-w^{3} \mathrm{G}_{5}^{\circ} \\
a & { }^{5} \mathrm{D}_{3}-y^{3} \mathrm{D}_{3}^{\circ} \\
a^{3} & { }^{3} \mathrm{~F}_{2}-x^{5} \mathrm{D}_{3}^{\circ} \\
a & { }^{5} \mathrm{P}_{1}-x^{5} \mathrm{D}_{0}^{0} \\
a^{3} \mathrm{G}_{3}-v^{3} \mathrm{D}_{3}^{\circ}\end{array}$ & $\begin{array}{r}200 \\
20 \\
6 \\
150 \\
60\end{array}$ & $\begin{array}{l}3605.640 \\
3606.153 \\
3607.414 \\
3608.729 \\
3609.102\end{array}$ & $\begin{array}{l}27726.42 \\
27722.47 \\
27712.78 \\
27702.69 \\
27699.82\end{array}$ & $\begin{array}{ll}a & 5 \mathrm{P}_{1}-z^{3} \mathrm{P}_{1}^{\circ} \\
a^{3} \mathrm{~B}_{3}-y & \mathrm{~F}_{3}^{5} \mathrm{~F}_{3}^{\circ} \\
a^{3} \mathrm{D}_{3}-y & \mathrm{D}_{2}^{\circ} \\
a^{5} \mathrm{D}_{2}-y^{3} \mathrm{D}_{3}^{\circ} \\
a^{5} \mathrm{~F}_{4}-z^{5} \mathrm{~F}_{3}^{\circ}\end{array}$ \\
\hline $\begin{array}{r}10 \\
6 \\
30 \\
200 \\
50\end{array}$ & $\begin{array}{l}3547.760 \\
3549.632 \\
3549.737 \\
3550.271 \\
3550.635\end{array}$ & $\begin{array}{l}28178.75 \\
28163.89 \\
28163.06 \\
28158.82 \\
28155.93\end{array}$ & 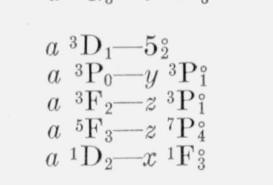 & $\begin{array}{r}4 \\
5 \\
150 \\
2 \\
200\end{array}$ & $\begin{array}{l}3612.934 \\
3616.056 \\
3616.954 \\
3618.570 \\
3619.201\end{array}$ & $\begin{array}{l}27670.44 \\
27646.55 \\
27639.69 \\
27627.35 \\
27622.53\end{array}$ & $\begin{array}{l}c{ }^{3} \mathrm{P}_{2}-39_{1}^{\circ} \\
a{ }^{1} \mathrm{G}_{4}-x^{3} \mathrm{~F}_{4}^{\circ} \\
b{ }^{3} \mathrm{~F}_{4}-y^{3} \mathrm{D}_{3}^{\circ} \\
c{ }^{3} \mathrm{~F}_{2}-55_{2}^{\circ} \\
a^{3} \mathrm{G}_{4}-1_{4}^{\circ} \\
a^{3} \mathrm{H}_{5}-w^{3} \mathrm{G}_{4}^{\circ}\end{array}$ \\
\hline $\begin{array}{r}10 \\
20 \\
200 \\
10 \\
2\end{array}$ & $\begin{array}{l}3551.220 \\
3553.672 \\
3553.850 \\
3554.274 \\
3555.827\end{array}$ & $\begin{array}{l}28151.29 \\
28131.87 \\
28130.46 \\
28127.11 \\
28114.82\end{array}$ & $\begin{array}{l}a{ }^{1} \mathrm{D}_{2}-y{ }^{5} \mathrm{~S}_{2} \\
a^{3} \mathrm{G}_{4}-z{ }^{1} \mathrm{~F}_{3}^{\circ} \\
a^{3} \mathrm{P}_{1}-y{ }^{3} \mathrm{P}_{1} \\
a^{5} \mathrm{D}_{0}-y{ }^{3} \mathrm{D}_{1}^{\circ} \\
b^{3} \mathrm{G}_{5}-62_{4}^{\circ}\end{array}$ & $\begin{array}{c}150 \\
60 \\
50 \\
10 h \\
300\end{array}$ & $\begin{array}{l}3620.288 \\
3623.634 \\
3623.694 \\
3623.855 \\
3625.197\end{array}$ & $\begin{array}{l}27614.24 \\
27588.74 \\
27588.28 \\
27587.06 \\
27576.85\end{array}$ & $\begin{array}{lll}b & { }^{3} \mathrm{P}_{2}-x & { }^{3} \mathrm{~F}_{3} \\
b & { }^{3} \mathrm{~F}_{3}-y^{3} \mathrm{~F}_{4} \\
b & { }^{3} \mathrm{P}_{0}-z & { }^{1} \mathrm{P}_{1}^{\circ} \\
a & { }^{1} \mathrm{H}_{5}-24_{5}^{\circ} \\
a & { }^{3} \mathrm{P}_{2}-x & { }^{5} \mathrm{D}_{1}^{\circ}\end{array}$ \\
\hline $\begin{array}{r}6 \\
125 \\
125 \\
4 \\
50\end{array}$ & $\begin{array}{l}3556.393 \\
3556.634 \\
3557.062 \\
3559.643 \\
3559.883\end{array}$ & $\begin{array}{l}28110.35 \\
28108.44 \\
28105.06 \\
28084.68 \\
28082.79\end{array}$ & $\begin{array}{lll}b & { }^{3} \mathrm{P}_{2}-z & { }^{1} \mathrm{D}_{2}^{\circ} \\
a & { }^{3} \mathrm{P}_{1}-y & { }^{3} \mathrm{P}_{0}^{0} \\
a^{3} \mathrm{H}_{4}-x & { }^{1} \mathrm{~F}_{3}^{\circ} \\
a & { }^{1} \mathrm{P}_{1}-33_{2}^{\circ} \\
a^{3} \mathrm{P}_{2}-y{ }^{5} \mathrm{P}_{3}^{\circ}\end{array}$ & $\begin{array}{r}300 \\
100 \\
20 \\
150 \\
20\end{array}$ & $\begin{array}{l}\text { 3626. } 740 \\
3627.285 \\
3631.414 \\
3631.710 \\
3632.412\end{array}$ & $\begin{array}{l}27565.11 \\
27560.97 \\
27529.63 \\
27527.39 \\
27522.07\end{array}$ & $\begin{array}{l}a{ }^{3} \mathrm{H}_{4}-y{ }^{3} \mathrm{H}_{i} \\
a^{3} \mathrm{G}_{3}-x{ }^{3} \mathrm{~F}_{3} \\
c{ }^{3} \mathrm{~F}_{3}-v{ }^{3} \mathrm{G}_{4} \\
a{ }^{3} \mathrm{~F}_{4}-z{ }^{5} \mathrm{P}_{3}^{\circ} \\
a^{3} \mathrm{D}_{1}-x^{3} \mathrm{P}_{2}^{\circ}\end{array}$ \\
\hline $\begin{array}{l}20 \\
30 \\
40 H \\
100 H \\
60\end{array}$ & $\begin{array}{l}3560.029 \\
3560.175 \\
3561.570 \\
3561.900 \\
3562.618\end{array}$ & $\begin{array}{l}28081.64 \\
28080.49 \\
28069.49 \\
28066.89 \\
28061.23\end{array}$ & $\begin{array}{l}a^{3} \mathrm{H}_{5}-x^{5} \mathrm{~F}_{5}^{\circ} \\
a{ }^{5} \mathrm{~F}_{1}-z^{5} \mathrm{~S}_{2}^{\circ} \\
a^{3} \mathrm{G}_{5}-y^{3} \mathrm{G}_{4}^{\circ} \\
b^{3} \mathrm{P}_{0}-w^{3} \mathrm{D}_{1} \\
a^{5} \mathrm{D}_{2}-z^{3} \mathrm{P}_{2}^{\circ}\end{array}$ & $\begin{array}{c}150 \\
1000 R \\
150 \\
250 \\
150\end{array}$ & $\begin{array}{l}3633.916 \\
3634.931 \\
3635.512 \\
3637.461 \\
3638.011\end{array}$ & $\begin{array}{l}27510.68 \\
27503.00 \\
27498.61 \\
27483.87 \\
27479.72\end{array}$ & $\begin{array}{l}a^{3} \mathrm{H}_{4}-x^{5} \mathrm{~F}_{4}^{\circ} \\
a{ }^{5} \mathrm{~F}_{3}-z^{7} \mathrm{~F}_{4}^{\circ} \\
a \\
a^{5} \mathrm{P}_{1}-z^{3} \mathrm{P}_{2}^{\circ} \\
a^{3} \mathrm{G}_{3}-x^{3} \mathrm{~F}_{2}^{\circ} \\
a^{3}{ }^{3} \mathrm{D}_{2}-w^{3} \mathrm{D}_{2}^{\circ}\end{array}$ \\
\hline $\begin{array}{r}20 \\
20 \\
10 \\
100 \\
30\end{array}$ & $\begin{array}{l}3563.148 \\
3563.611 \\
3563.827 \\
3564.364 \\
3564.504\end{array}$ & $\begin{array}{l}28057.06 \\
28053.41 \\
28051.71 \\
28047.49 \\
28046.38\end{array}$ & $\begin{array}{l}a^{3} \mathrm{G}_{3}-z{ }^{1} \mathrm{D}_{2}^{\circ} \\
a^{3} \mathrm{D}_{2}-y{ }^{3} \mathrm{~S}_{1} \\
b{ }^{3} \mathrm{~F}_{3}-y{ }^{5} \mathrm{P}_{3}^{\circ} \\
a^{5} \mathrm{P}_{1}-x{ }^{5} \mathrm{D}_{2}^{\circ} \\
a^{3} \mathrm{H}_{6}-y{ }^{1} \mathrm{H}_{5}^{\circ} \\
c{ }^{3} \mathrm{P}_{1}-58_{1}^{\circ}\end{array}$ & $\begin{array}{r}1 \\
150 \\
2 \\
4 \\
40\end{array}$ & $\begin{array}{l}3639.140 \\
3640.638 \\
3644.582 \\
3644.852 \\
3645.674\end{array}$ & $\begin{array}{l}27471.19 \\
27459.89 \\
27430.17 \\
27428.14 \\
27421.96\end{array}$ & $\begin{array}{l}c{ }^{3} \mathrm{P}_{2}-34_{3}^{\circ} \\
a^{3} \mathrm{G}_{4}-y^{3} \mathrm{G}_{4}^{\circ} \\
b^{3} \mathrm{H}_{5}-v^{3} \mathrm{G}_{4}^{\circ} \\
a^{3} \mathrm{H}_{6}-z^{3} \mathrm{I}_{5}^{\circ} \\
b^{3} \mathrm{~F}_{4}-x^{5} \mathrm{D}_{4}^{\circ}\end{array}$ \\
\hline $\begin{array}{r}150 \\
60 \\
100 \\
10 \\
50\end{array}$ & $\begin{array}{l}3564.570 \\
3564.809 \\
3567.157 \\
3567.751 \\
3568.527\end{array}$ & $\begin{array}{l}28045.87 \\
28043.99 \\
28025.53 \\
28020.86 \\
28014.77\end{array}$ & 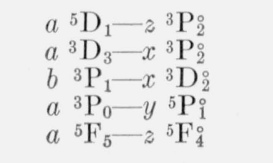 & $\begin{array}{r}150 \\
2 \\
2 \\
175\end{array}$ & $\begin{array}{l}3646.111 \\
3649.196 \\
3649.538 \\
3650.324\end{array}$ & $\begin{array}{l}27418.67 \\
27395.49 \\
27392.92 \\
27387.03\end{array}$ & $\begin{array}{l}a^{3} \mathrm{G}_{4}-y^{3} \mathrm{G}_{3}^{\circ} \\
c{ }^{3} \mathrm{~F}_{2}-53_{1}^{\circ} \\
a{ }^{1} \mathrm{D}_{2}-4{ }_{3}^{\circ} \\
c^{3} \mathrm{P}_{2}-33_{2}^{\circ} \\
a^{3}{ }^{3} \mathrm{~F}_{3}-y^{5} \mathrm{~F}_{4}^{\circ}\end{array}$ \\
\hline $\begin{array}{r}1000 \\
60 \\
150 \\
10 \\
200\end{array}$ & $\begin{array}{l}3570.606 \\
3571.767 \\
3572.022 \\
3573.666 \\
3574.601\end{array}$ & $\begin{array}{l}27998.46 \\
27989.36 \\
27987.36 \\
27974.48 \\
27967.17\end{array}$ & $\begin{array}{l}a{ }^{3} \mathrm{H}_{6}-y{ }^{3} \mathrm{H}_{6}^{\circ} \\
a{ }^{5} \mathrm{P}_{3}-y{ }^{3} \mathrm{D}_{3}^{\circ} \\
a{ }^{3} \mathrm{P}_{1}-y{ }^{5} \mathrm{P}_{1}^{\circ} \\
a^{3} \mathrm{H}_{4}-w^{5} \mathrm{D}_{3}^{\circ} \\
a^{5} \mathrm{D}_{3}-x^{5} \mathrm{D}_{4}^{\circ}\end{array}$ & $\begin{array}{r}100 \\
7 \\
2 \\
30 \\
150\end{array}$ & $\begin{array}{l}3652.311 \\
3652.488 \\
3652.667 \\
3653.698 \\
3654.405\end{array}$ & $\begin{array}{l}27372.13 \\
27370.80 \\
27369.46 \\
27361.74 \\
27356.44\end{array}$ & $\begin{array}{l}a{ }^{5} \mathrm{~F}_{2}-z^{7} \mathrm{~F}_{1} \\
b{ }^{3} \mathrm{P}_{2}-x^{3} \mathrm{D}_{1}^{\circ} \\
a{ }^{1} \mathrm{H}_{5}-21_{5}^{\circ} \\
a^{3} \mathrm{D}_{2}-z{ }^{1} \mathrm{P}_{1} \\
a^{3} \mathrm{H}_{5}-y{ }^{1} \mathrm{H}_{5}^{\circ}\end{array}$ \\
\hline
\end{tabular}


TABLE 3-Continued

\begin{tabular}{|c|c|c|c|c|c|c|c|}
\hline Intensity & $\begin{array}{l}\text { Wave- } \\
\text { length }\end{array}$ & $\begin{array}{c}\text { Wave } \\
\text { number }\end{array}$ & Term combination & Intensity & $\begin{array}{l}\text { Wave- } \\
\text { length }\end{array}$ & $\begin{array}{c}\text { Wave } \\
\text { number }\end{array}$ & Term combination \\
\hline $\begin{array}{r}4 \\
6 \\
150 \\
30\end{array}$ & $\begin{array}{c}\text { A } \\
\text { 3655. } 936 \\
3655.984 \\
3657.169 \\
3659.470\end{array}$ & $\begin{array}{c}c m^{-1} \\
27344.99 \\
27344.63 \\
27335.77 \\
27318.58\end{array}$ & $\begin{array}{l}a{ }^{5} \mathrm{P}_{1}-y^{3} \mathrm{D}_{2}^{\circ} \\
a^{3} \mathrm{H}_{4}-4_{3}^{\circ} \\
a \quad \mathrm{~F}_{3}-z^{5} \mathrm{~F}_{2}^{\circ} \\
a^{3} \mathrm{D}_{3}-u^{3} \mathrm{D}_{2}^{\circ} \\
b^{1} \mathrm{D}_{2}-60_{1}^{\circ}\end{array}$ & $\begin{array}{c}2000 R \\
50 \\
40 \\
100 \\
4\end{array}$ & $\begin{array}{c}\text { A } \\
3730.432 \\
3730.592 \\
3730.899 \\
3732.020 \\
3732.744\end{array}$ & $\begin{array}{l}\quad c m^{-1} \\
26798.93 \\
26797.78 \\
26795.57 \\
26787.52 \\
26782.33\end{array}$ & 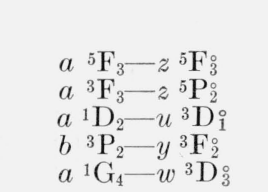 \\
\hline $\begin{array}{c}60 \\
2000 R \\
50 \\
800 \\
2\end{array}$ & $\begin{array}{l}3660.813 \\
3661.364 \\
3661.570 \\
3663.378 \\
3667.982\end{array}$ & $\begin{array}{l}27308.56 \\
27304.45 \\
27302.91 \\
27289.44 \\
27255.19\end{array}$ & $\begin{array}{l}a^{3} \mathrm{H}_{5}-y{ }^{3} \mathrm{H}_{6}^{\circ} \\
a{ }^{5} \mathrm{~F}_{4}-z^{3} \mathrm{G}_{5}^{\circ} \\
b{ }^{3} \mathrm{P}_{2}-z{ }^{1} \mathrm{~F}_{3}^{\circ} \\
a^{5} \mathrm{D}_{4}-y^{5} \mathrm{~F}_{5}^{\circ} \\
a^{1} \mathrm{D}_{2}-3_{1}^{\circ}\end{array}$ & $\begin{array}{r}100 \\
40 \\
2 \\
300 \\
50\end{array}$ & $\begin{array}{l}3733.041 \\
3735.020 \\
3736.823 \\
3737.407 \\
3737.758\end{array}$ & $\begin{array}{l}26780.20 \\
26766.01 \\
26753.09 \\
26748.91 \\
26746.40\end{array}$ & $\begin{array}{l}a{ }^{3} \mathrm{~F}_{2}-y^{5} \mathrm{~F}_{2}^{\circ} \\
a^{3} \mathrm{H}_{4}-w^{3} \mathrm{G}_{4}^{\circ} \\
b^{3} \mathbf{F}_{2}-x^{5} \mathrm{D}_{1}^{\circ} \\
a^{5} \mathrm{D}_{2}-y^{5} \mathrm{~F}_{3}^{\circ} \\
a^{5} \mathrm{D}_{0}-y^{5} \mathbf{F}_{1}^{\circ}\end{array}$ \\
\hline $\begin{array}{r}30 \\
600 \\
80 \\
10 \\
100\end{array}$ & $\begin{array}{l}3668.742 \\
3669.546 \\
3671.212 \\
3672.059 \\
3672.378\end{array}$ & $\begin{array}{l}27249.54 \\
27243.57 \\
27231.21 \\
27224.92 \\
27222.56\end{array}$ & $\begin{array}{l}a^{3} \mathrm{G}_{3}-2{ }^{1} \mathrm{~F}_{3}^{\circ} \\
a^{3} \mathrm{G}_{5}-y^{3} \mathrm{G}_{5}^{\circ} \\
a^{5} \mathrm{D}_{3}-y{ }^{5} \mathrm{~F}_{3}^{\circ} \\
a^{3} \mathrm{H}_{4}-x^{5} \mathrm{~F}_{5}^{\circ} \\
a^{3} \mathrm{P}_{1}-y{ }^{5} \mathrm{P}_{2}^{\circ}\end{array}$ & $\begin{array}{c}150 \\
200 \\
250 \\
10 \\
1000 R\end{array}$ & $\begin{array}{l}3738.630 \\
3738.914 \\
3739.470 \\
3741.006 \\
3742.287\end{array}$ & $\begin{array}{l}26740.16 \\
26738.13 \\
26734.16 \\
26723.18 \\
26714.03\end{array}$ & $\begin{array}{l}a^{3} \mathrm{G}_{3}-1_{4}^{\circ} \\
a^{3} \mathrm{H}_{5}-z^{3} \mathrm{I}_{5}^{\circ} \\
a^{3} \mathrm{G}_{3}-y^{3} \mathrm{~F}_{2}^{\circ} \\
a^{3} \mathrm{P}_{2}-z^{3} \mathrm{P}_{1}^{\circ} \\
a^{5} \mathrm{~F}_{2}-z^{5} \mathrm{~F}_{2}^{\circ}\end{array}$ \\
\hline $\begin{array}{r}2 \\
2 \\
40 \\
2 \\
60\end{array}$ & $\begin{array}{l}3674.002 \\
3674.634 \\
3675.253 \\
3676.376 \\
3676.663\end{array}$ & $\begin{array}{l}27210.53 \\
27205.85 \\
27201.26 \\
27192.96 \\
27190.84\end{array}$ & $\begin{array}{ll}c & 3 \mathrm{~F}_{4}-42_{4}^{\circ} \\
a^{1} \mathrm{H}_{5}-19_{4}^{\circ} \\
b{ }^{3} \mathrm{P}_{1}-x^{3} \mathrm{~F}_{2}^{\circ} \\
a & { }^{5} \mathrm{P}_{3}-y^{5} \mathrm{~F}_{2}^{\circ} \\
a^{3} \mathrm{D}_{1}-y^{1} \mathrm{D}_{2}^{\circ}\end{array}$ & $\begin{array}{r}400 \\
60 \\
40 \\
125 \\
125\end{array}$ & $\begin{array}{l}3742.798 \\
3743.328 \\
3743.956 \\
3744.219 \\
3744.396\end{array}$ & $\begin{array}{l}26710.39 \\
26706.60 \\
26702.12 \\
26700.25 \\
26698.99\end{array}$ & $\begin{array}{l}a^{3} \mathrm{H}_{6}-z^{3} \mathrm{I}_{7}^{\circ} \\
a^{3} \mathrm{D}_{3}-v^{3} \mathrm{D}_{2}^{\circ} \\
a^{3} \mathrm{D}_{2}-z{ }^{1} \mathrm{D}_{2}^{\circ} \\
a^{5} \mathrm{P}_{3}-y{ }^{5} \mathrm{~F}_{4}^{\circ} \\
a^{3} \mathrm{H}_{5}-x^{3} \mathrm{G}_{4}^{\circ}\end{array}$ \\
\hline $\begin{array}{r}40 \\
10 \\
10 \\
200 \\
10\end{array}$ & $\begin{array}{l}3676.952 \\
3677.976 \\
3678.056 \\
3678.314 \\
3680.052\end{array}$ & $\begin{array}{l}27188.70 \\
27181.13 \\
27180.54 \\
27178.63 \\
27165.80\end{array}$ & $\begin{array}{l}a{ }^{1} \mathrm{D}_{2}-x^{3} \mathrm{P}_{2}^{\circ} \\
a^{5} \mathrm{D}_{2}-y^{5} \mathrm{~F}_{1}^{\circ} \\
a^{1} \mathrm{G}_{4}-v^{3} \mathrm{D}_{3}^{\circ} \\
a^{5} \mathrm{~F}_{2}-z^{7} \mathrm{~F}_{3}^{\circ} \\
a^{5} \mathrm{D}_{1}-y^{5} \mathrm{~F}_{1}^{\circ}\end{array}$ & $\begin{array}{r}500 \\
50 \\
40 \\
60 \\
150\end{array}$ & $\begin{array}{l}3745.592 \\
3746.218 \\
3751.856 \\
3752.787 \\
3753.546\end{array}$ & $\begin{array}{l}26690.46 \\
26686.00 \\
26645.90 \\
26639.29 \\
26633.91\end{array}$ & $\begin{array}{l}a^{3} \mathrm{G}_{5}-z^{3} \mathrm{H}_{6}^{\circ} \\
b^{3} \mathrm{~F}_{4}-y^{5} \mathrm{~F}_{3}^{\circ} \\
a^{3} \mathrm{H}_{4}-w^{3} \mathrm{G}_{5}^{\circ} \\
a^{1} \mathrm{H}_{5}-14_{4}^{\circ} \\
a^{3} \mathrm{G}_{4}-y^{3} \mathrm{G}_{5}^{\circ}\end{array}$ \\
\hline $\begin{array}{r}1 \\
16 \\
40 \\
100 \\
20\end{array}$ & $\begin{array}{l}3682.644 \\
3683.573 \\
3685.050 \\
3685.944 \\
3686.588\end{array}$ & $\begin{array}{l}27146.68 \\
27139.83 \\
27128.95 \\
27122.37 \\
27117.63\end{array}$ & $\begin{array}{l}a^{3} \mathrm{H}_{4}-w^{5} \mathrm{D}_{4}^{\circ} \\
b^{3} \mathrm{~F}_{2}-z^{3} \mathrm{~S}_{1}^{\circ} \\
a^{3} \mathrm{D}_{1}-u^{3} \mathrm{D}_{1}^{\circ} \\
b{ }^{3} \mathrm{P}_{2}-x^{3} \mathrm{D}_{3}^{\circ} \\
b^{3} \mathrm{H}_{4}-v^{3} \mathrm{G}_{5}^{\circ}\end{array}$ & $\begin{array}{r}200 \\
50 \\
300 \\
200 \\
400\end{array}$ & $\begin{array}{l}3755.094 \\
3755.728 \\
3755.937 \\
3759.838 \\
3760.019\end{array}$ & $\begin{array}{l}26622.93 \\
26618.43 \\
26616.95 \\
26589.33 \\
26588.05\end{array}$ & $\begin{array}{l}a^{3} \mathbf{F}_{2}-y{ }^{5} \mathbf{F}_{3}^{\circ} \\
a{ }^{5} \mathbf{P}_{1}-y{ }^{5} \mathbf{F}_{1}^{\circ} \\
a^{3} \mathbf{G}_{4}-y^{3} \mathbf{F}_{3}^{\circ} \\
a^{5} \mathrm{D}_{4}-z^{5} \mathbf{P}_{3}^{\circ} \\
a^{5} \mathbf{F}_{1}-z^{5} \mathbf{F}_{1}^{\circ}\end{array}$ \\
\hline $\begin{array}{r}4 \\
50 \\
10 \\
200 \\
60\end{array}$ & $\begin{array}{l}\text { 3688. } 776 \\
3693.589 \\
3693.634 \\
3696.583 \\
3697.857\end{array}$ & $\begin{array}{l}27101.55 \\
27066.24 \\
27065.91 \\
27044.31 \\
27035.00\end{array}$ & $\begin{array}{ll}a & { }^{1} \mathrm{H}_{5}-17_{4}^{\circ} \\
a^{3} \mathrm{G}_{5}-z^{3} \mathrm{H}_{4}^{\circ} \\
c^{3} \mathrm{~B}_{2}-49_{1}^{\circ} \\
a^{3} \mathrm{P}_{2}-x^{5} \mathrm{D}_{2}^{\circ} \\
b^{3} \mathrm{P}_{1}-x^{3} \mathrm{D}_{1}^{\circ}\end{array}$ & $\begin{array}{r}200 \\
50 \\
10 \\
150 \\
2\end{array}$ & $\begin{array}{l}3761.511 \\
3764.037 \\
3765.808 \\
3767.353 \\
3770.463 .\end{array}$ & $\begin{array}{l}26577.51 \\
26559.67 \\
26547.18 \\
26536.30 \\
26514.41\end{array}$ & $\begin{array}{l}a^{3} \mathrm{G}_{3}-y^{3} \mathrm{G}_{4}^{\circ} \\
a^{1} \mathrm{G}_{4}-x^{3} \mathrm{~F}_{3}^{\circ} \\
a{ }^{1} \mathrm{P}_{1}-15_{1}^{\circ} \\
a^{3} \mathrm{G}_{3}-y^{3} \mathrm{G}_{3}^{\circ} \\
c^{3} \mathrm{~F}_{3}-43_{3}^{\circ}\end{array}$ \\
\hline $\begin{array}{r}150 \\
60 \\
2 \\
50 \\
6\end{array}$ & $\begin{array}{l}3700.980 \\
3701.312 \\
3701.833 \\
3702.228 \\
3702.920\end{array}$ & $\begin{array}{l}27012.18 \\
27009.76 \\
27005.96 \\
27003.08 \\
26998.03\end{array}$ & $\begin{array}{l}a{ }^{3} \mathrm{H}_{4}-y{ }^{3} \mathrm{H}_{5}^{\circ} \\
a{ }^{5} \mathrm{~F}_{1}-z z^{7} \mathrm{~F}_{0}^{\circ} \\
a \\
{ }^{1} \mathrm{P}_{1}-w^{3} \mathrm{~F}_{2} \\
a \\
{ }^{5} \mathrm{P}_{2}-z{ }^{5} \mathrm{P}_{1}^{\circ} \\
c^{3} \mathrm{~F}_{3}-50_{2}^{\circ}\end{array}$ & $\begin{array}{r}2 \\
60 \\
400 \\
60\end{array}$ & $\begin{array}{l}3772.386 \\
3773.175 \\
3777.588 \\
3777.758\end{array}$ & $\begin{array}{l}26500.89 \\
26495.35 \\
26464.40 \\
26463.21\end{array}$ & $\begin{array}{ll}c & { }^{3} \mathrm{~F}_{4}-31_{4}^{\circ} \\
a & { }^{3} \mathrm{P}_{2}-z^{3} \mathrm{P}_{2}^{\circ} \\
a & { }^{5} \mathrm{~F}_{1}-z^{5} \mathrm{D}_{0}^{\circ} \\
b & { }^{3} \mathrm{~F}_{3}-z^{3} \mathrm{P}_{2}^{\circ} \\
a^{1}{ }^{1} \mathrm{D}_{2}-u^{3} \mathrm{D}_{2}^{\circ}\end{array}$ \\
\hline $\begin{array}{r}60 \\
30 \\
3 \\
4 \\
20\end{array}$ & $\begin{array}{l}3703.203 \\
3705.347 \\
3705.400 \\
3709.089 \\
3710.302\end{array}$ & $\begin{array}{l}26995.97 \\
26980.35 \\
26979.96 \\
26953.13 \\
26944.32\end{array}$ & $\begin{array}{l}a{ }^{3} \mathrm{P}_{2}-y^{3} \mathrm{D}_{1}^{\circ} \\
a{ }^{5} \mathrm{~F}_{2}-z \\
{ }^{5} \mathrm{~F}_{1} \\
a{ }^{5} \mathrm{~F}_{1}-z{ }^{7} \mathrm{~F}_{1}^{\circ} \\
a^{3} \mathrm{D}_{2}-x^{3} \mathrm{D}_{2}^{\circ} \\
b^{3} \mathrm{H}_{4}-v^{3} \mathrm{G}_{4}^{\circ}\end{array}$ & $\begin{array}{r}150 \\
10 \\
50 \\
150 \\
30\end{array}$ & $\begin{array}{l}3778.711 \\
3779.426 \\
3779.969 \\
3781.171 \\
3781.660\end{array}$ & $\begin{array}{l}26456.54 \\
26451.53 \\
26447.73 \\
26439.32 \\
26435.91\end{array}$ & $\begin{array}{ll}a^{3} \mathrm{G}_{4}-z^{3} \mathrm{H}_{4}^{\circ} \\
b{ }^{3} \mathrm{P}_{1}-y^{3} \mathrm{~F}_{2}^{\circ} \\
a{ }^{3} \mathrm{P}_{0}-x{ }^{5} \mathrm{D}_{1}^{\circ} \\
a^{1} \mathrm{H}_{5}-x{ }^{1} \mathbf{H}_{5}^{\circ} \\
c^{3} \mathrm{~F}_{2}-41_{2}^{\circ}\end{array}$ \\
\hline $\begin{array}{r}100 \\
50 \\
125 \\
150 \\
300\end{array}$ & $\begin{array}{l}3712.299 \\
3714.644 \\
3715.559 \\
3716.173 \\
3716.998\end{array}$ & $\begin{array}{l}26929.82 \\
26912.82 \\
26906.20 \\
26901.75 \\
26895.78\end{array}$ & $\begin{array}{l}a^{1} \mathrm{D}_{2}-x^{3} \mathrm{G}_{3}^{\circ} \\
a^{5} \mathrm{~F}_{1}-z^{7} \mathrm{~F}_{2}^{\circ} \\
a^{5} \mathrm{D}_{2}-y{ }^{5} \mathrm{~F}_{2}^{\circ} \\
a^{3} \mathrm{~F}_{4}-y{ }^{5} \mathrm{D}_{4}^{\circ} \\
a^{5}{ }^{5} \mathrm{D}_{3}-y{ }^{5} \mathrm{~F}_{4}^{\circ}\end{array}$ & $\begin{array}{c}120 \\
6 \\
1000 \\
1500 R \\
2\end{array}$ & $\begin{array}{l}3782.749 \\
3784.749 \\
3786.065 \\
3790.521 \\
3793.916\end{array}$ & $\begin{array}{l}26428.30 \\
26414.33 \\
26405.15 \\
26374.11 \\
26350.51\end{array}$ & $\begin{array}{ll}d^{3} \mathrm{~F}_{4}-62_{4}^{\circ} \\
a^{3} \mathrm{P}_{1}-x^{5} \mathrm{D}_{1}^{\circ} \\
a^{5} \mathrm{~F}_{2}-z^{5} \mathrm{D}_{1}^{\circ} \\
a^{5} \mathrm{~F}_{3}-z^{5} \mathrm{D}_{2}^{\circ} \\
b^{3} \mathbf{F}_{4}-y^{5} \mathrm{~F}_{4}^{\circ}\end{array}$ \\
\hline $\begin{array}{r}40 \\
50 \\
300 \\
4 \\
10\end{array}$ & $\begin{array}{l}3717.674 \\
3718.400 \\
3719.325 \\
3721.929 \\
3722.303\end{array}$ & $\begin{array}{l}26890.89 \\
26885.64 \\
26878.95 \\
26860.15 \\
26857.45\end{array}$ & $\begin{array}{l}a^{5} \mathrm{D}_{1}-y{ }^{5} \mathrm{~F}_{2}^{\circ} \\
a^{3} \mathrm{~F}_{4}-y{ }^{5} \mathrm{D}_{3}^{\circ} \\
a^{3} \mathrm{H}_{4}-x^{3} \mathrm{G}_{3}^{\circ} \\
c^{3} \mathrm{~F}_{4}-36_{5}^{\circ} \\
a^{1} \mathrm{D}_{2}-y^{1} \mathrm{D}_{2}^{\circ}\end{array}$ & $\begin{array}{l}125 \\
100 \\
250 \\
3000 R \\
3000 R\end{array}$ & $\begin{array}{l}3794.924 \\
3795.185 \\
3798.054 \\
3798.899 \\
3799.353\end{array}$ & $\begin{array}{l}26343.51 \\
26341.70 \\
26321.80 \\
26315.95 \\
26312.80\end{array}$ & $\begin{array}{lll}a & { }^{5} \mathrm{P}_{1}-y^{5} \mathrm{~F}_{2}^{\circ} \\
a & { }^{3} \mathrm{P}_{2}-y^{3} \mathrm{D}_{2}^{\circ} \\
a & 5 \mathrm{~F}_{1}-z^{5} \mathrm{~F}_{2}^{\circ} \\
a & 5 \mathrm{~F}_{4}-z^{5} \mathrm{D}_{3}^{\circ} \\
a^{5} \mathrm{~F}_{5}-z^{5} \mathrm{D}_{4}^{\circ}\end{array}$ \\
\hline $\begin{array}{c}150 \\
50 \\
300 \\
5000 R \\
9000 R\end{array}$ & $\begin{array}{l}3724.967 \\
3725.482 \\
3726.096 \\
3726.926 \\
3728.026\end{array}$ & $\begin{array}{l}\text { 26838. } 24 \\
26834.53 \\
26830.11 \\
26824.14 \\
26816.22\end{array}$ & $\begin{array}{ll}a & { }^{5} \mathrm{P}_{2}-z^{5} \mathrm{P}_{2}^{\circ} \\
a & { }^{3} \mathrm{P}_{0}-z^{3} s_{1}^{\circ} \\
a^{3} \mathrm{G}_{5}-z^{1} \mathrm{G}_{4} \\
a^{5} \mathrm{~F}_{4}-z^{5} \mathrm{~F}_{4}^{\circ} \\
a^{5}{ }^{5} \mathrm{~F}_{5}-z^{5} \mathrm{~F}_{5}^{\circ}\end{array}$ & $\begin{array}{l}150 \\
100 \\
100 \\
125 \\
300\end{array}$ & $\begin{array}{l}3800.261 \\
3803.191 \\
3805.432 \\
3808.689 \\
3812.739\end{array}$ & $\begin{array}{l}26306.51 \\
26286.25 \\
26270.77 \\
26248.30 \\
26220.42\end{array}$ & 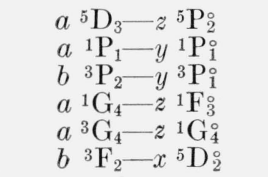 \\
\hline
\end{tabular}


Table 3-Continued

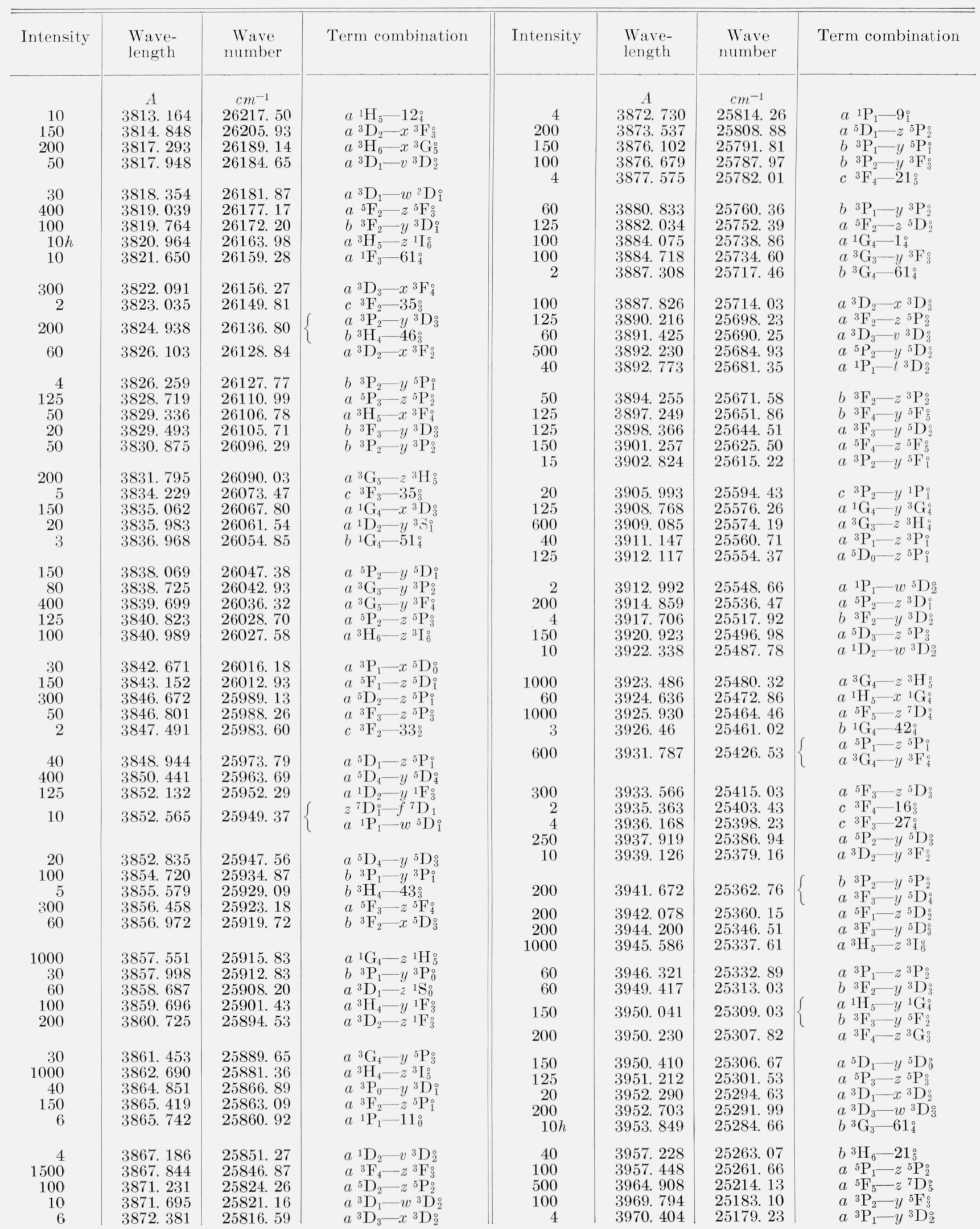


Table 3-Continued

\begin{tabular}{|c|c|c|c|c|c|c|c|}
\hline Intensity & $\begin{array}{l}\text { Wave- } \\
\text { length }\end{array}$ & $\begin{array}{c}\text { Wave } \\
\text { number }\end{array}$ & Term combination & Intensity & $\begin{array}{l}\text { Wave- } \\
\text { length }\end{array}$ & $\begin{array}{c}\text { Wave } \\
\text { number }\end{array}$ & Term combination \\
\hline $\begin{array}{r}150 \\
2 \\
500 \\
500 \\
60\end{array}$ & $\begin{array}{c}\text { A } \\
\text { 3974. } 504 \\
3976.729 \\
3978.449 \\
3979.420 \\
3981.895\end{array}$ & $\begin{array}{c}c m^{-1} \\
25153.26 \\
25139.18 \\
25128.32 \\
25122.18 \\
25106.57\end{array}$ & $\begin{array}{l}a{ }^{5} \mathrm{D}_{3}-y^{5} \mathrm{D}_{2}^{\circ} \\
b^{3} \mathrm{H}_{4}-31_{4}^{\circ} \\
a^{5} \mathrm{P}_{2}-z^{3} \mathrm{~F}_{2}^{\circ} \\
a^{5} \mathrm{~F}_{4}-z^{5} \mathrm{D}_{4}^{\circ} \\
c^{3} \mathrm{~F}_{3}-22_{3}^{\circ}\end{array}$ & $\begin{array}{r}60 \\
200 \\
20 \\
200 \\
600\end{array}$ & $\begin{array}{c}\text { A } \\
4062.854 \\
4062.989 \\
4063.325 \\
4064.105 \\
4064.456\end{array}$ & $\begin{array}{c}c m^{-1} \\
24606.29 \\
24605.47 \\
24603.44 \\
24598.72 \\
24596.59\end{array}$ & $\begin{array}{l}a{ }^{1} \mathrm{H}_{5}-y{ }^{3} \mathrm{H}_{4}^{\circ} \\
b{ }^{3} \mathrm{P}_{1}-z^{3} \mathrm{~S}_{1} \\
c{ }^{3} \mathrm{~F}_{2}-w^{3} \mathrm{~F}_{3}^{\circ} \\
a^{5} \mathrm{D}_{0}-y{ }^{5} \mathrm{D}_{1}^{\circ} \\
a^{5} \mathrm{D}_{3}-z^{3} \mathrm{~F}_{2}^{\circ}\end{array}$ \\
\hline $\begin{array}{r}50 \\
20 \\
1000 \\
400 \\
60\end{array}$ & $\begin{array}{l}\text { 3982. } 217 \\
\text { 3984. } 678 \\
\text { 3984. } 862 \\
\text { 3987. } 797 \\
3989.194\end{array}$ & $\begin{array}{l}25104.54 \\
25089.04 \\
25087.88 \\
25069.41 \\
25060.63\end{array}$ & $\begin{array}{ll}b^{3} \mathrm{H}_{4}-30_{4}^{\circ} \\
b{ }^{3} \mathrm{P}_{0}-y^{3} \mathrm{P}_{1}^{\circ} \\
a & { }^{3} \mathrm{~F}_{3}-z^{3} \mathrm{~F}_{2}^{\circ} \\
a^{3} \mathrm{D}_{3}-x^{3} \mathrm{~F}_{3}^{\circ} \\
b^{3} \mathrm{P}_{2}-y^{5} \mathrm{P}_{3}^{\circ}\end{array}$ & $\begin{array}{r}6 \\
200 \\
600 \\
150 \\
20\end{array}$ & $\begin{array}{l}\text { 4066. } 222 \\
4067.610 \\
4068.367 \\
4071.398 \\
4071.865\end{array}$ & $\begin{array}{l}24585.91 \\
24577.52 \\
24572.95 \\
24554.65 \\
24551.84\end{array}$ & $\begin{array}{l}z z^{7} \mathrm{D}_{2}^{\circ}-f^{7} \mathrm{D}_{2}^{\circ} \\
a^{3} \mathrm{D}_{3}-x^{3} \mathrm{D}_{3}^{\circ} \\
a^{1}{ }^{1} \mathrm{G}_{4}-z^{3} \mathrm{H}_{4}^{\circ} \\
b{ }^{3} \mathrm{P}_{2}-x^{5} \mathrm{D}_{1}^{\circ} \\
a^{1}{ }^{1} \mathrm{H}_{5}-x^{5} \mathrm{~F}_{4}^{\circ}\end{array}$ \\
\hline $\begin{array}{r}4 \\
100 \\
10 \\
200 \\
100\end{array}$ & $\begin{array}{l}\text { 3991. } 793 \\
3993.532 \\
3994.562 \\
3995.982 \\
3996.504\end{array}$ & $\begin{array}{l}25044.32 \\
25033.41 \\
25026.96 \\
25018.06 \\
25014.80\end{array}$ & $\begin{array}{l}c{ }^{3} \mathrm{P}_{2}-8_{1}^{\circ} \\
a^{5} \mathrm{D}_{2}-y^{5} \mathrm{D}_{1}^{\circ} \\
b{ }^{3} \mathrm{P}_{1}-y{ }^{5} \mathrm{P}_{2}^{\circ} \\
a^{5} \mathrm{D}_{1}-y^{5} \mathrm{D}_{1}^{\circ} \\
a^{5} \mathrm{D}_{2}-z^{5} \mathrm{P}_{3}^{\circ}\end{array}$ & $\begin{array}{r}125 \\
60 \\
3 \\
800 \\
100\end{array}$ & $\begin{array}{l}\text { 4072. } 996 \\
\text { 4073. } 116 \\
\text { 4075. } 978 \\
4076.730 \\
4079.277\end{array}$ & $\begin{array}{l}24545.02 \\
24544.30 \\
24527.06 \\
24522.54 \\
24507.23\end{array}$ & $\begin{array}{l}a{ }^{3} \mathrm{~F}_{2}-y{ }^{5} \mathrm{D}_{2}^{\circ} \\
a^{3} \mathrm{G}_{3}-y{ }^{3} \mathrm{~F}_{4}^{\circ} \\
c^{3} \mathrm{~F}_{3}-w^{3} \mathrm{~F}_{3}^{\circ} \\
a^{5} \mathrm{D}_{2}-z^{3} \mathrm{D}_{1}^{\circ} \\
a^{5} \mathrm{D}_{1}-z^{3} \mathrm{D}_{1}^{\circ}\end{array}$ \\
\hline $\begin{array}{r}4 \\
4 \\
4 \\
6 \\
200\end{array}$ & $\begin{array}{l}3999.488 \\
4000.103 \\
4000.550 \\
4005.089 \\
4005.646\end{array}$ & $\begin{array}{l}24996.13 \\
24992.29 \\
24989.50 \\
24961.18 \\
24957.71\end{array}$ & 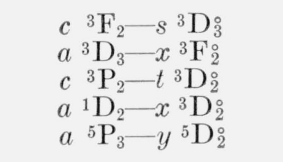 & $\begin{array}{r}1500 \\
100 \\
4 \\
100 \\
300\end{array}$ & $\begin{array}{l}4080.599 \\
4082.789 \\
4083.868 \\
4085.353 \\
4085.432\end{array}$ & $\begin{array}{l}24499.29 \\
24486.15 \\
24479.68 \\
24470.78 \\
24470.31\end{array}$ & $\begin{array}{l}a{ }^{3} \mathrm{~F}_{4}-z^{3} \mathrm{D}_{3}^{\circ} \\
a^{3} \mathrm{P}_{0}-y^{5} \mathrm{~F}_{1}^{\circ} \\
b^{3} \mathrm{G}_{4}-v^{3} \mathrm{G}_{5}^{\circ} \\
a{ }^{5} \mathrm{P}_{1}-y^{5} \mathrm{I}_{1}^{\circ} \\
a^{3} \mathrm{D}_{1}-x^{3} \mathrm{~F}_{2}^{\circ}\end{array}$ \\
\hline $\begin{array}{r}150 \\
100 \\
125 \\
20 \\
125\end{array}$ & $\begin{array}{l}\text { 4006. } 602 \\
4007.533 \\
4008.266 \\
4011.732 \\
4013.502\end{array}$ & $\begin{array}{l}24951.75 \\
24945.96 \\
24941.39 \\
24919.85 \\
24908.86\end{array}$ & $\begin{array}{ll}b & { }^{3} \mathbf{F}_{4}-z{ }^{5} \mathbf{P}_{3}^{\circ} \\
b & { }^{3} \mathbf{P}_{0}-y{ }^{5} \mathbf{P}_{1} \\
b & { }^{3} \mathrm{P}_{2}-z{ }^{3} \mathbf{S}_{1}^{\circ} \\
c & { }^{3} \mathbf{F}_{3}-s^{3} \mathbf{D}_{3}^{\circ} \\
a^{5} \mathbf{D}_{4}-z^{3} \mathbf{F}_{3}^{\circ}\end{array}$ & $\begin{array}{r}40 \\
150 \\
10 \\
250 \\
1000\end{array}$ & $\begin{array}{l}\text { 4088. } 370 \\
4091.062 \\
4093.792 \\
4097.022 \\
4097.787\end{array}$ & $\begin{array}{l}24452.72 \\
24436.63 \\
24420.34 \\
24401.09 \\
24396.53\end{array}$ & $\begin{array}{l}a{ }^{3} \mathrm{P}_{1}-y^{5} \mathrm{~F}_{1}^{\circ} \\
a^{1}{ }^{1} \mathrm{D}_{2}-w^{3} \mathrm{D}_{3}^{\circ} \\
b^{3} \mathrm{H}_{4}-21_{5}^{\circ} \\
a^{5} \mathrm{P}_{3}-z^{3} \mathrm{~F}_{2}^{\circ} \\
a^{3}{ }^{3} \mathrm{~F}_{2}-z^{3} \mathrm{D}_{1}^{\circ}\end{array}$ \\
\hline $\begin{array}{r}40 \\
40 \\
20 \\
60 \\
150\end{array}$ & $\begin{array}{l}\text { 4013. } 736 \\
4014.153 \\
4016.740 \\
4019.551 \\
4020.994\end{array}$ & $\begin{array}{l}24907.40 \\
24904.82 \\
24888.78 \\
24871.37 \\
24862.45\end{array}$ & $\begin{array}{l}a{ }^{3} \mathbf{F}_{2}-y{ }^{5} \mathrm{D}_{1}^{\circ} \\
c^{3} \mathbf{F}_{2}-w^{3} \mathbf{F}_{2}^{\circ} \\
a^{3} \mathbf{F}_{2}-z^{5} \mathrm{P}_{3}^{\circ} \\
a^{5} \mathrm{D}_{3}-y^{5} \mathrm{D}_{4}^{\circ} \\
a^{3} \mathrm{D}_{2}-y^{3} \mathrm{P}_{1}^{\circ}\end{array}$ & $\begin{array}{r}150 \\
40 \\
300 \\
150 \\
2\end{array}$ & $\begin{array}{l}\text { 4100. } 365 \\
4101.226 \\
4101.742 \\
4102.281 \\
4104.049\end{array}$ & $\begin{array}{l}24381.19 \\
24376.07 \\
24373.01 \\
24369.81 \\
24359.31\end{array}$ & $\begin{array}{l}a{ }^{5} \mathrm{~F}_{3}-z^{7} \mathrm{D}_{2}^{\circ} \\
a^{3} \mathrm{H}_{5}-z^{1} \mathrm{H}_{5}^{\circ} \\
a^{5} \mathrm{D}_{2}-y{ }^{5} \mathrm{D}_{3}^{\circ} \\
a^{5} \mathrm{D}_{4}-z^{3} \mathrm{G}_{3}^{\circ} \\
b^{3} \mathrm{~F}_{2}-y^{5} \mathrm{~F}_{3}^{\circ}\end{array}$ \\
\hline $\begin{array}{r}800 \\
30 \\
600 \\
50 \\
100\end{array}$ & $\begin{array}{l}4022.168 \\
4022.680 \\
4023.832 \\
4024.300 \\
4024.689\end{array}$ & $\begin{array}{l}24855.19 \\
24852.03 \\
24844.91 \\
24842.02 \\
24839.62\end{array}$ & 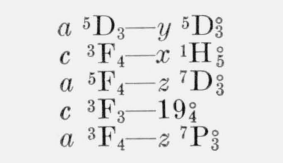 & $\begin{array}{r}50 \\
2 \\
200 \\
100 \\
60\end{array}$ & $\begin{array}{l}\text { 4105. } 909 \\
\text { 4106. } 217 \\
\text { 4107. } 837 \\
4108.055 \\
4109.640\end{array}$ & $\begin{array}{l}24348.27 \\
24346.45 \\
24336.84 \\
24335.55 \\
24326.17\end{array}$ & $\begin{array}{ll}a & { }^{5} \mathrm{P}_{2}-z^{3} \mathrm{~F}_{3}^{\circ} \\
b^{3} \mathrm{G}_{5}-v^{3} \mathrm{G}_{4}^{\circ} \\
a^{1}{ }^{1} \mathrm{G}_{4}-z^{1} \mathrm{G}_{4}^{\circ} \\
a^{3}{ }^{3} \mathrm{G}_{5}-x^{5} \mathrm{D}_{4}^{\circ} \\
b^{3} \mathrm{~F}_{4}-y^{5} \mathrm{D}_{4}^{\circ}\end{array}$ \\
\hline $\begin{array}{r}2 \\
10 \\
40 \\
200 \\
200\end{array}$ & $\begin{array}{l}4025.465 \\
4026.492 \\
4028.434 \\
4031.003 \\
4032.207\end{array}$ & $\begin{array}{l}24834.83 \\
24828.50 \\
24816.53 \\
24800.71 \\
24793.31\end{array}$ & $\begin{array}{ll}a & { }^{1} \mathrm{D}_{2}-v^{3} \mathrm{D}_{3}^{\circ} \\
c & { }^{3} \mathrm{~F}_{3}-w^{3} \mathrm{~F}_{2}^{\circ} \\
b & { }^{3} \mathrm{~F}_{3}-y^{5} \mathrm{~F}_{4}^{\circ} \\
a^{3} \mathrm{~F}_{4}-z^{5} \mathrm{G}_{4}^{\circ} \\
a^{5} \mathrm{~F}_{2}-z^{5} \mathrm{D}_{3}^{\circ}\end{array}$ & $\begin{array}{r}2000 \\
150 \\
60 \\
4 \\
200\end{array}$ & $\begin{array}{l}\text { 4112. } 741 \\
\text { 4113. } 380 \\
\text { 4114. } 128 \\
\text { 4117. } 76 \\
\text { 4118. } 500\end{array}$ & $\begin{array}{l}24307.83 \\
24304.05 \\
24299.63 \\
24278.20 \\
24273.84\end{array}$ & $\begin{array}{l}a{ }^{3} \mathrm{~F}_{3}-z^{3} \mathrm{~F}_{3}^{\circ} \\
a^{3} \mathrm{D}_{1}-x^{3} \mathrm{D}_{1}^{\circ} \\
a{ }^{5} \mathrm{P}_{2}-z z^{7} \mathrm{P}_{2}^{\circ} \\
z^{7} \mathrm{D}_{2}^{\circ}-f^{7} \mathrm{D}_{2} \\
a^{5} \mathrm{~F}_{4}-z^{7} \mathrm{D}_{4}^{\circ}\end{array}$ \\
\hline $\begin{array}{r}50 \\
1 \\
80 \\
300\end{array}$ & $\begin{array}{l}4032.509 \\
4033.724 \\
4037.738 \\
4039.215\end{array}$ & $\begin{array}{l}24791.45 \\
24783.98 \\
24759.35 \\
24750.29\end{array}$ & $\begin{array}{l}b{ }^{3} \mathrm{~F}_{2}-y{ }^{5} \mathrm{~F}_{1}^{\circ} \\
a^{3} \mathrm{H}_{4}-v^{3} \mathrm{D}_{3}^{\circ} \\
b^{3} \mathrm{H}_{4}-25_{3}^{\circ} \\
a{ }^{5} \mathrm{P}_{1}-y{ }^{5} \mathrm{D}_{0}^{\circ} \\
a^{1} \mathrm{G}_{4}-y^{3} \mathrm{G}_{5}^{\circ}\end{array}$ & $\begin{array}{r}6 \\
300 \\
100 \\
5 \\
300\end{array}$ & $\begin{array}{l}\text { 4119. } 458 \\
\text { 4120. } 986 \\
\text { 4121. } 126 \\
\text { 4122. } 786 \\
\text { 4123. } 061\end{array}$ & $\begin{array}{l}24268.19 \\
24259.19 \\
24258.37 \\
24248.60 \\
24246.99\end{array}$ & $\begin{array}{l}c{ }^{3} \mathrm{P}_{2}-x{ }^{1} \mathrm{~F}_{3}^{\circ} \\
a{ }^{3} \mathrm{~F}_{3}-z^{7} \mathrm{P}_{2}^{\circ} \\
a{ }^{3} \mathrm{P}_{2}-z^{5} \mathrm{P}_{2}^{\circ} \\
a^{3} \mathrm{D}_{3}-1_{4}^{\circ} \\
a^{3}{ }^{3} \mathrm{~F}_{2}-y^{5} \mathrm{D}_{3}^{\circ}\end{array}$ \\
\hline $\begin{array}{r}50 \\
6 \\
20 \\
2 \\
2\end{array}$ & $\begin{array}{l}4040.474 \\
4041.259 \\
4041.980 \\
4044.262 \\
4044.710\end{array}$ & $\begin{array}{l}24742.58 \\
24737.78 \\
24733.36 \\
24719.41 \\
24716.67\end{array}$ & $\begin{array}{l}b^{3}{ }^{3} \mathrm{H}_{5}-19_{4}^{\circ} \\
c \quad{ }^{3} \mathrm{~F}_{3}-17_{4}^{\circ} \\
a^{1} \mathrm{G}_{4}-y^{3} \mathrm{~F}_{3}^{\circ} \\
a^{3}{ }^{3} \mathrm{D}_{2}-y^{5} \mathrm{P}_{1}^{\circ} \\
b^{1}{ }^{1} \mathrm{G}_{4}-30_{4}^{\circ}\end{array}$ & $\begin{array}{r}75 \\
30 \\
200 \\
200\end{array}$ & $\begin{array}{l}\text { 4123. } 801 \\
4126.407 \\
4127.439 \\
4127.869\end{array}$ & $\begin{array}{l}24242.63 \\
24227.32 \\
24221.27 \\
24218.74\end{array}$ & $\begin{array}{ll}a & { }^{3} \mathrm{D}_{3}-y^{3} \mathrm{~F}_{2}^{\circ} \\
b & { }^{1} \mathrm{G}_{4}-23_{3}^{\circ} \\
b & { }^{3} \mathrm{~F}_{3}-z^{5} \mathrm{P}_{2}^{\circ} \\
a & { }^{5} \mathrm{~F}_{3}-z \\
b^{5} & { }^{5} \mathrm{D}_{4}^{\circ}-x^{5} \mathrm{D}_{1}^{\circ}\end{array}$ \\
\hline $\begin{array}{r}150 \\
20 \\
150 \\
1000 \\
200\end{array}$ & $\begin{array}{l}4045.770 \\
4046.883 \\
4049.418 \\
4051.402 \\
4052.194\end{array}$ & $\begin{array}{l}24710.19 \\
24703.40 \\
24687.93 \\
24675.84 \\
24671.02\end{array}$ & $\begin{array}{l}a{ }^{1} \mathrm{D}_{2}-z{ }^{1} \mathrm{D}_{2}^{\circ} \\
c^{3} \mathrm{~F}_{2}-16_{3}^{\circ} \\
a^{3} \mathrm{D}_{2}-y{ }^{3} \mathrm{P}_{2}^{\circ} \\
a{ }^{5} \mathrm{P}_{3}-y{ }^{5} \mathrm{D}_{4}^{\circ} \\
a^{5} \mathrm{D}_{2}-y{ }^{5} \mathrm{D}_{2}^{\circ}\end{array}$ & $\begin{array}{r}2 \\
20 \\
1 \\
50 \\
200\end{array}$ & $\begin{array}{l}\text { 4128. } 664 \\
\text { 4131. } 215 \\
\text { 4133. } 614 \\
\text { 4134. } 854 \\
\text { 4137. } 223\end{array}$ & $\begin{array}{l}24214.08 \\
24199.13 \\
24185.08 \\
24177.83 \\
24163.99\end{array}$ & $\begin{array}{l}a{ }^{1} \mathrm{D}_{2}-x^{3} \mathrm{~F}_{3}^{\circ} \\
a^{3} \mathrm{H}_{5}-1_{4}^{\circ} \\
c{ }^{3} \mathrm{~F}_{2}-y{ }^{1} \mathrm{P}_{1}^{\circ} \\
a^{3} \mathrm{P}_{1}-y{ }^{5} \mathrm{~F}_{2}^{\circ} \\
a^{5}{ }^{5} \mathrm{P}_{2}-z^{3} \mathrm{D}_{2}^{\circ}\end{array}$ \\
\hline $\begin{array}{r}600 \\
10 \\
1 \\
25 \\
4\end{array}$ & $\begin{array}{l}4054.050 \\
4054.713 \\
4057.562 \\
4058.852 \\
4059.427\end{array}$ & $\begin{array}{l}24659.73 \\
24655.69 \\
24638.38 \\
24630.55 \\
24627.06\end{array}$ & $\begin{array}{l}a{ }^{5} \mathrm{P}_{3}-y{ }^{5} \mathrm{D}_{3}^{\circ} \\
a^{5} \mathrm{D}_{1}-y{ }^{5} \mathrm{D}_{2}^{\circ} \\
b^{3} \mathrm{H}_{5}-17_{4}^{\circ} \\
b^{3}{ }^{3} \mathrm{H}_{4}-23_{3}^{\circ} \\
c^{3} \mathrm{~F}_{3}-16_{3}^{\circ}\end{array}$ & $\begin{array}{l}4 \\
2 \\
4 \\
2 H\end{array}$ & $\begin{array}{l}\text { 4137. } 948 \\
\text { 4139. } 192 \\
\text { 4140. } 276 \\
4141.856\end{array}$ & $\begin{array}{l}24159.76 \\
24152.49 \\
24146.17 \\
24136.96\end{array}$ & $\begin{array}{l}z^{7} \mathrm{D}_{4}^{\circ}-f^{7} \mathrm{D}_{4}^{\circ} \\
b^{3} \mathrm{H}_{4}-17_{4}^{\circ} \\
z^{3} \mathrm{G}_{4}^{\circ}-\mathrm{E}_{4} \\
z^{7} \mathrm{D}_{2}^{\circ}-e^{7} \mathrm{D}_{1} \\
a^{1} \mathrm{D}_{2}-x^{3} \mathrm{~F}_{2}^{\circ}\end{array}$ \\
\hline
\end{tabular}


Table 3-Continued

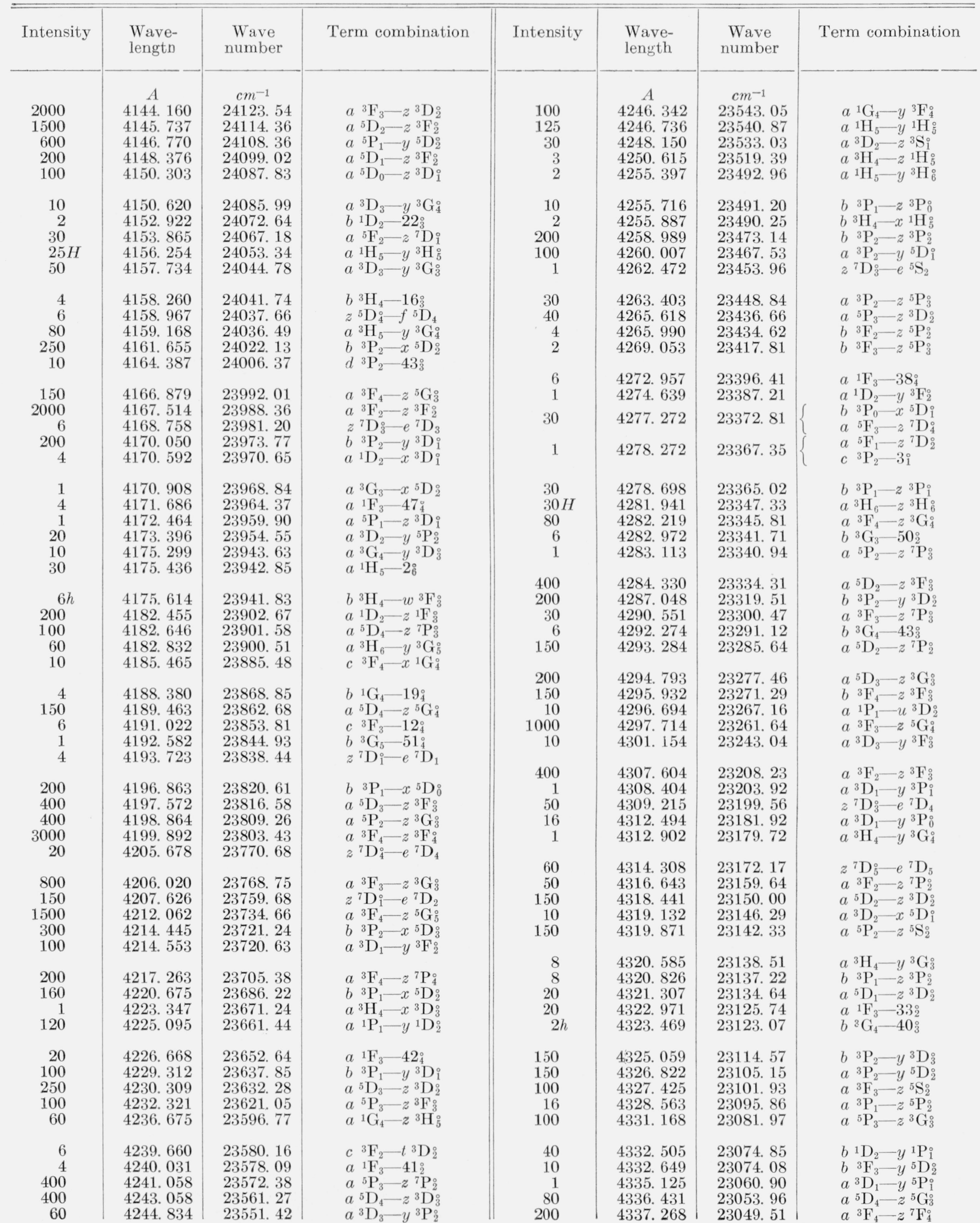


TABle 3 -Continued

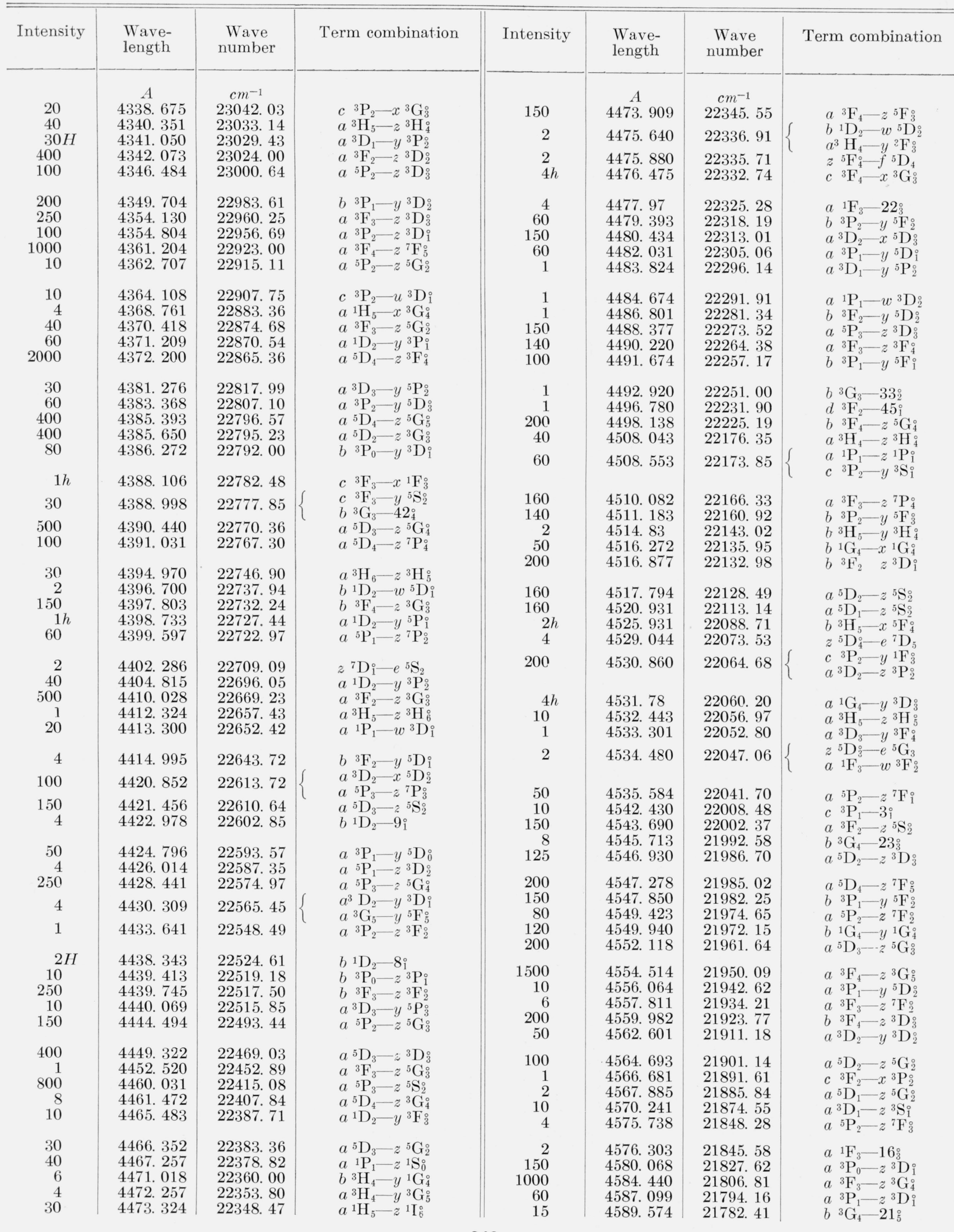


TABLE 3 -Continued

\begin{tabular}{|c|c|c|c|c|c|c|c|}
\hline Intensity & $\begin{array}{l}\text { Wave- } \\
\text { length }\end{array}$ & $\begin{array}{l}\text { Wave } \\
\text { number }\end{array}$ & Term combination & Intensity & $\begin{array}{l}\text { Wave- } \\
\text { length }\end{array}$ & $\begin{array}{l}\text { Wave } \\
\text { number }\end{array}$ & Term combination \\
\hline $\begin{array}{r}2 h \\
200 \\
100 \\
6 \\
200\end{array}$ & $\begin{array}{c}A \\
4590.727 \\
4591.097 \\
4591.546 \\
4592.005 \\
4592.513\end{array}$ & $\begin{array}{c}c m^{-1} \\
21776.94 \\
21775.19 \\
21773.06 \\
21770.88 \\
21768.47\end{array}$ & $\begin{array}{l}z^{7} \mathrm{D}_{5}^{\circ}-e^{5} \mathrm{G}_{6} \\
a^{3} \mathrm{~F}_{2}-z^{5} \mathrm{G}_{2}^{\circ} \\
a^{5} \mathrm{D}_{3}-z^{3} \mathrm{~F}_{4}^{\circ} \\
z^{5} \mathrm{G}_{2}^{\circ}-f^{7} \mathrm{D}_{1} \\
a^{3} \mathrm{P}_{2}-z^{3} \mathrm{~F}_{3}^{\circ}\end{array}$ & $\begin{array}{r}10 \\
1 \\
2 \\
1 \\
1000\end{array}$ & $\begin{array}{l}\text { A } \\
4695.253 \\
4698.700 \\
4700.261 \\
4705.16 \\
4709.482\end{array}$ & $\begin{array}{c}c m^{-1} \\
21292.15 \\
21276.53 \\
21269.46 \\
21247.32 \\
21227.82\end{array}$ & $\begin{array}{l}z^{5} \mathrm{D}_{2}^{\circ}-e^{3} \mathrm{D}_{2} \\
z^{5} \mathrm{~F}_{4}-e^{5} \mathrm{P}_{3} \\
z^{5} \mathrm{~S}_{2}^{\circ}-\mathrm{D}_{3} \\
a^{5} \mathrm{P}_{3}-z^{7} \mathrm{~F}_{2}^{\circ} \\
b^{3} \mathrm{~F}_{4}-z^{3} \mathrm{~F}_{4}^{\circ}\end{array}$ \\
\hline $\begin{array}{r}10 \\
10 \\
1 \\
150 \\
1\end{array}$ & $\begin{array}{l}4592.995 \\
4593.195 \\
4594.641 \\
4596.703 \\
4597.331\end{array}$ & $\begin{array}{l}21766.19 \\
21765.24 \\
21758.39 \\
21748.63 \\
21745.66\end{array}$ & $\begin{array}{l}a{ }^{5} \mathrm{P}_{3}-z^{5} \mathrm{G}_{3}^{\circ} \\
a{ }^{1} \mathrm{P}_{1}-x^{3} \mathrm{D}_{2}^{\circ} \\
a^{7}{ }^{7} \mathrm{D}_{5}^{\circ}-e^{5} \mathrm{D}_{4} \\
b^{1}{ }^{1} \mathrm{D}_{2}-x^{1} \mathbf{F}_{3}^{\circ} \\
a^{1} \mathbf{F}_{3}-w^{3} \mathbf{F}_{3}^{\circ}\end{array}$ & $\begin{array}{l}20 \\
80 \\
16 h \\
10 \\
1\end{array}$ & $\begin{array}{l}4710.968 \\
4711.988 \\
4712.502 \\
4713.370 \\
4714.834\end{array}$ & $\begin{array}{l}21221.12 \\
21216.53 \\
21214.21 \\
21210.31 \\
21203.72\end{array}$ & $\begin{array}{l}z^{5} \mathrm{D}_{3}^{\circ}-e^{3} \mathrm{G}_{4} \\
c^{3} \mathrm{P}_{1}-u^{3} \mathrm{D}_{2}^{\circ} \\
z^{5} \mathrm{D}_{3}^{\circ}-e^{5} \mathrm{D}_{2} \\
z^{5} \mathrm{D}_{2}^{\circ}-e^{3} \mathrm{G}_{3} \\
d^{3} \mathrm{~F}_{4}-35_{3}^{\circ}\end{array}$ \\
\hline $\begin{array}{r}1 \\
500 \\
150 \\
30 \\
150\end{array}$ & $\begin{array}{l}4597.697 \\
4599.082 \\
4601.757 \\
4602.820 \\
4605.671\end{array}$ & $\begin{array}{l}21743.93 \\
21737.38 \\
21724.74 \\
21719.73 \\
21706.28\end{array}$ & 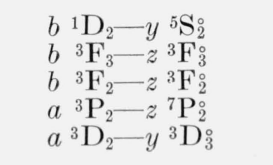 & $\begin{array}{r}6 \\
100 \\
6 \\
120 \\
1\end{array}$ & $\begin{array}{l}4715.615 \\
4716.049 \\
4718.840 \\
4720.920 \\
4721.848\end{array}$ & $\begin{array}{l}21200.21 \\
21198.26 \\
21185.72 \\
21176.39 \\
21172.23\end{array}$ & $\begin{array}{l}a^{3} \mathrm{H}_{4}-z^{3} \mathrm{H}_{5}^{\circ} \\
b^{3} \mathrm{~F}_{3}-z^{3} \mathrm{G}_{3}^{\circ} \\
b^{3} \mathrm{G}_{3}-19_{4}^{\circ} \\
a^{3} \mathrm{D}_{3}-x^{5} \mathrm{D}_{3}^{\circ} \\
b^{3} \mathrm{G}_{3}-w^{3} \mathrm{~F}_{2}\end{array}$ \\
\hline $\begin{array}{r}2 \\
10 \\
60 \\
2 \\
2\end{array}$ & $\begin{array}{l}4608.673 \\
4610.492 \\
4612.328 \\
4615.425 \\
4616.093\end{array}$ & $\begin{array}{l}21692.14 \\
21683.59 \\
21674.95 \\
21660.41 \\
21657.28\end{array}$ & $\begin{array}{l}z^{7} \mathrm{D}_{5}^{\circ}-f^{5} \mathrm{~F}_{5} \\
a^{1} \mathrm{H}_{5}-x^{3} \mathrm{G}_{5}^{\circ} \\
a^{5} \mathrm{D}_{3}-z^{7} \mathrm{P}_{4}^{\circ} \\
a^{1} \mathrm{D}_{2}-y{ }^{5} \mathrm{P}_{3}^{\circ} \\
b^{3} \mathrm{H}_{4}-y{ }^{3} \mathrm{H}_{4}^{\circ}\end{array}$ & $\begin{array}{r}10 \\
40 \\
4 \\
300 \\
150\end{array}$ & $\begin{array}{l}4723.226 \\
4724.799 \\
4727.584 \\
4731.323 \\
4733.319\end{array}$ & $\begin{array}{l}21166.05 \\
21159.00 \\
21146.54 \\
21129.83 \\
21120.92\end{array}$ & $\begin{array}{ll}c & { }^{3} \mathrm{~F}_{2}-u^{3} \mathrm{D}_{2}^{\circ} \\
b & { }^{3} \mathrm{~F}_{4}-z^{5} \mathrm{G}_{5}^{\circ} \\
a^{3} \mathrm{H}_{4}-y & { }^{3} \mathrm{~F}_{4}^{\circ} \\
b & { }^{3} \mathrm{~F}_{4}-z^{7} \mathrm{P}_{4}^{\circ} \\
a^{5} \mathrm{P}_{3}-z^{7} \mathrm{~F}_{3}^{\circ}\end{array}$ \\
\hline $\begin{array}{l}30 \\
4 h \\
6 \\
8 \\
4\end{array}$ & $\begin{array}{l}\text { 4617. } 662 \\
\text { 4618. } 024 \\
\text { 4618. } 799 \\
4624.322 \\
4624.464\end{array}$ & $\begin{array}{l}21649.92 \\
21648.22 \\
21644.59 \\
21618.74 \\
21618.07\end{array}$ & $\begin{array}{ll}a & 5 \mathrm{P}_{2}-z^{5} \mathrm{~F}_{\mathrm{i}}^{\circ} \\
d & { }^{3} \mathbf{F}_{3}-47_{4}^{\circ} \\
d & { }^{3} \mathbf{F}_{4}-43_{3}^{\circ} \\
b^{3} \mathrm{G}_{4}-19_{4}^{\circ} \\
b^{1}{ }^{1} \mathrm{D}_{2}-w^{5} \mathrm{D}_{3}^{\circ}\end{array}$ & $\begin{array}{r}300 \\
10 \\
125 \\
2\end{array}$ & $\begin{array}{l}4733.520 \\
4740.332 \\
4743.028 \\
4743.670\end{array}$ & $\begin{array}{l}21120.02 \\
21089.67 \\
21077.68 \\
21074.83\end{array}$ & $\begin{array}{l}a{ }^{5} \mathrm{P}_{3}-z^{3} \mathrm{G}_{4} \\
a^{3} \mathrm{D}_{1}-x{ }^{5} \mathrm{D}_{0}^{\circ} \\
c^{3} \mathrm{~F}_{3}-u^{3} \mathrm{D}_{2}^{\circ} \\
b^{3} \mathrm{H}_{5}-y{ }^{1} \mathrm{H}_{5}^{\circ} \\
a^{5} \mathrm{P}_{2}-z^{5} \mathrm{D}_{1}^{\circ}\end{array}$ \\
\hline $\begin{array}{r}20 \\
30 \\
10 \\
1\end{array}$ & $\begin{array}{l}4626.035 \\
4628.337 \\
4630.442 \\
4631.74\end{array}$ & $\begin{array}{l}21610.73 \\
21599.98 \\
21590.16 \\
21584.11\end{array}$ & $\begin{array}{ll}c & { }^{3} \mathrm{P}_{1}-y{ }^{1} \mathrm{D}_{2}^{\circ} \\
c & { }^{3} \mathrm{P}_{2}-w^{3} \mathrm{D}_{2}^{\circ} \\
b^{3} \mathrm{H}_{5}-y & { }^{3} \mathrm{H}_{5}^{\circ} \\
z & { }^{5} \mathbf{F}_{4}^{\circ}-e^{5} \mathrm{G}_{3} \\
a^{3} \mathrm{P}_{2}-z^{3} \mathrm{D}_{2}^{\circ}\end{array}$ & $\begin{array}{r}2 \\
4 \\
1 \\
2 \\
30\end{array}$ & $\begin{array}{l}4743.984 \\
4744.654 \\
4745.859 \\
4749.803 \\
4751.015\end{array}$ & $\begin{array}{l}21073.44 \\
21070.46 \\
21065.11 \\
21047.62 \\
21042.25\end{array}$ & $\begin{array}{l}c{ }^{3} \mathrm{P}_{2}-x^{3} \mathrm{D}_{2}^{\circ} \\
z^{7} \mathrm{P}_{3}^{\circ}-\mathrm{D}_{3} \\
b^{3} \mathrm{P}_{1}-z^{5} \mathrm{P}_{1} \\
z^{5} \mathrm{D}_{2}^{5}-e^{5} \mathrm{D}_{1} \\
a^{5} \mathrm{D}_{1}-z^{7} \mathrm{~F}_{0}^{\circ}\end{array}$ \\
\hline $\begin{array}{c}2 \\
300 \\
20 h \\
20 \\
30\end{array}$ & $\begin{array}{l}4633.173 \\
4635.682 \\
4638.419 \\
4639.318 \\
4640.983\end{array}$ & $\begin{array}{l}21577.44 \\
21565.76 \\
21553.04 \\
21548.86 \\
21541.13\end{array}$ & 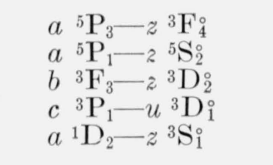 & $\begin{array}{c}4 h \\
30 \\
6 \\
250 \\
500\end{array}$ & $\begin{array}{l}4751.454 \\
4752.140 \\
4753.844 \\
4756.244 \\
4757.844\end{array}$ & $\begin{array}{l}21040.30 \\
21037.27 \\
21029.73 \\
21019.12 \\
21012.05\end{array}$ & $\begin{array}{l}z^{7} \mathrm{~F}_{2}^{\circ}-f^{7} \mathrm{D}_{2} \\
z^{5} \mathrm{D}_{3}^{\circ}-f^{5} \mathrm{~F}_{4} \\
b^{3} \mathrm{H}_{5}-y{ }^{3} \mathrm{H}_{6}^{\circ} \\
a^{5} \mathrm{D}_{3}-z^{7} \mathrm{~F}_{4}^{\circ} \\
a^{5} \mathrm{D}_{4}-z^{3} \mathrm{G}_{5}^{\circ}\end{array}$ \\
\hline $\begin{array}{r}200 \\
6 \\
600 \\
60 \\
4\end{array}$ & $\begin{array}{l}4645.09 \\
4646.810 \\
4647.594 \\
4648.158 \\
4650.211\end{array}$ & $\begin{array}{l}21522.08 \\
21514.12 \\
21510.49 \\
21507.88 \\
21498.38\end{array}$ & 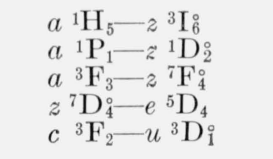 & $\begin{array}{r}6 \\
150 \\
4 \\
60 \\
300\end{array}$ & $\begin{array}{l}4761.533 \\
4764.406 \\
4766.647 \\
4767.153 \\
4769.306\end{array}$ & $\begin{array}{l}\text { 20995. } 77 \\
20983.11 \\
20973.24 \\
20971.02 \\
20961.55\end{array}$ & $\begin{array}{l}d{ }^{3} \mathrm{P}_{2}-t^{3} \mathrm{D}_{2}^{\circ} \\
z^{5} \mathrm{D}_{3}^{\circ}-e{ }^{3} \mathrm{D}_{3} \\
z^{7} \mathrm{~F}_{1}-f^{7} \mathrm{D}_{2} \\
b^{3} \mathrm{H}_{4}-x^{3} \mathrm{G}_{3} \\
a^{3} \mathrm{~F}_{4}-z^{5} \mathrm{D}_{3}^{\circ}\end{array}$ \\
\hline 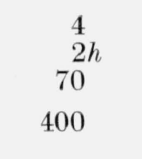 & $\begin{array}{l}4652.498 \\
4653.322 \\
4653.759 \\
4654.311\end{array}$ & $\begin{array}{l}21487.81 \\
21484.01 \\
21481.99 \\
21479.44\end{array}$ & $\begin{array}{l}a^{3} \mathrm{D}_{1}-x{ }^{5} \mathrm{D}_{1} \\
c{ }^{3} \mathrm{~F}_{3}-y \\
{ }^{1} \mathrm{D}_{2}^{\circ} \\
c{ }^{3} \mathrm{P}_{2}-{ }^{1} \mathrm{P}_{1}^{\circ} \\
a^{5} \mathrm{D}_{2}-z{ }^{5} \mathrm{G}_{3}^{\circ} \\
a^{5} \mathrm{P}_{3}-z^{7} \mathrm{P}_{4}^{\circ}\end{array}$ & $\begin{array}{r}125 \\
125 \\
15 \\
50 \\
20\end{array}$ & $\begin{array}{l}4773.155 \\
4774.004 \\
4781.112 \\
4781.770 \\
4783.300\end{array}$ & $\begin{array}{l}20944.65 \\
20940.92 \\
20909.79 \\
20906.91 \\
20900.23\end{array}$ & $\begin{array}{ll}b & { }^{3} \mathrm{~F}_{2}-z \\
a & { }^{3} \mathrm{~F}_{3}^{\circ} \\
a & { }^{1} \mathrm{P}_{1}-x{ }^{3} \mathrm{~F}_{2} \\
a^{3} \mathrm{D}_{2}-y & { }^{5} \mathrm{~F}_{2}^{\circ} \\
a^{3} \mathrm{D}_{1}-y{ }^{3} \mathrm{D}_{1}^{\circ} \\
b^{3} \mathrm{P}_{1}-z^{5} \mathrm{P}_{2}^{\circ}\end{array}$ \\
\hline $\begin{array}{r}50 \\
60 \\
4 \\
6 \\
20\end{array}$ & $\begin{array}{l}4654.780 \\
4656.415 \\
4660.640 \\
4662.254 \\
4662.494\end{array}$ & $\begin{array}{l}21477.28 \\
21469.74 \\
21450.28 \\
21442.85 \\
21441.75\end{array}$ & $\begin{array}{l}a^{3} \mathrm{D}_{3}-x^{5} \mathrm{D}_{2}^{\circ} \\
a^{3} \mathrm{~F}_{4}-z^{5} \mathrm{~F}_{4}^{\circ} \\
b^{3} \mathrm{G}_{3}-22_{3}^{\circ} \\
a^{5} \mathrm{D}_{3}-z^{7} \mathrm{~F}_{2}^{\circ} \\
z^{7} \mathrm{D}_{4}^{\circ}-f^{5} \mathrm{~F}_{5}\end{array}$ & $\begin{array}{c}150 \\
1 \\
10 \\
4 h \\
150\end{array}$ & $\begin{array}{l}4784.266 \\
4785.079 \\
4787.357 \\
4790.809 \\
4794.386\end{array}$ & $\begin{array}{l}20896.01 \\
20892.46 \\
20882.51 \\
20867.47 \\
20851.90\end{array}$ & $\begin{array}{ll}b & { }^{3} \mathrm{~F}_{2}-z^{7} \mathrm{P}_{2}^{\circ} \\
b^{3} \mathrm{G}_{5}-x & { }^{1} \mathrm{H}_{5}^{5} \\
z^{7} & { }^{7} \mathrm{~F}_{5}^{\circ}-f^{5} \mathrm{D}_{4} \\
z & { }^{5} \mathrm{~F}_{3}^{\circ}-e \\
a^{5}{ }^{5} \mathrm{D}_{2} \\
{ }^{5} \mathrm{D}_{3}-z^{5} \mathrm{~F}_{2}^{\circ}\end{array}$ \\
\hline $\begin{array}{r}100 \\
250 \\
6 \\
300 \\
40\end{array}$ & $\begin{array}{l}4669.139 \\
4669.973 \\
4671.368 \\
4674.638 \\
4681.408\end{array}$ & $\begin{array}{l}\text { 21411. } 23 \\
21407.41 \\
21401.02 \\
21386.05 \\
21355.12\end{array}$ & $\begin{array}{l}b{ }^{3} \mathrm{P}_{0}-y{ }^{5} \mathbf{F}_{1} \\
a^{5} \mathrm{D}_{4}-z{ }^{5} \mathbf{F}_{3}^{\circ} \\
b^{3}{ }^{3} \mathrm{P}_{2}-z{ }^{5} \mathrm{P}_{1}^{\circ} \\
a^{3} \mathrm{P}_{1}-z^{3} \mathbf{F}_{2}^{\circ} \\
c^{3} \mathbf{F}_{4}-y{ }^{1} \mathbf{F}_{3}^{\circ}\end{array}$ & $\begin{array}{r}150 \\
150 \\
60 \\
20 \\
200\end{array}$ & $\begin{array}{l}4795.575 \\
4798.450 \\
4801.180 \\
4802.91 \\
4804.887\end{array}$ & $\begin{array}{l}20846.73 \\
20834.24 \\
20822.39 \\
20814.89 \\
20806.33\end{array}$ & 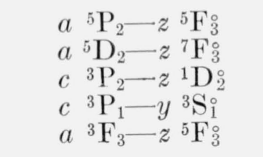 \\
\hline $\begin{array}{r}400 \\
300 \\
2 \\
400 \\
2 h\end{array}$ & $\begin{array}{l}4681.780 \\
4684.013 \\
4685.052 \\
4690.114 \\
4692.912\end{array}$ & $\begin{array}{l}21353.42 \\
21343.24 \\
21338.51 \\
21315.48 \\
21302.77\end{array}$ & $\begin{array}{l}a{ }^{3} \mathrm{~F}_{2}-z^{5} \mathrm{G}_{3}^{\circ} \\
a^{3} \mathrm{~F}_{3}-z^{5} \mathrm{~F}_{2}^{\circ} \\
a{ }^{5} \mathrm{P}_{1}-z^{5} \mathrm{G}_{2} \\
a^{5} \mathrm{D}_{3}-z^{3} \mathrm{G}_{4} \\
z^{5} \mathrm{D}_{3}^{\circ}-f^{5} \mathrm{~F}_{3}\end{array}$ & $\begin{array}{r}4 \\
20 \\
4 \\
6\end{array}$ & $\begin{array}{l}4807.633 \\
4809.714 \\
4810.693 \\
4812.225\end{array}$ & $\begin{array}{l}20794.45 \\
20785.45 \\
20781.22 \\
20774.60\end{array}$ & $\begin{array}{ll}z & { }^{5} \mathrm{~F}_{4}^{\circ}-f^{5} \mathrm{~F}_{3} \\
z & { }^{5} \mathrm{~F}_{3}^{\circ}-e^{3} \mathrm{G}_{3} \\
b^{1} \mathrm{D}_{2}-x & { }^{3} \mathrm{P}_{2}^{\circ} \\
a^{3} & { }^{3} \mathrm{D}_{3}-y{ }^{3} \mathrm{D}_{2}^{\circ} \\
a^{1} & { }^{1} \mathrm{P}_{1}-x^{3} \mathrm{D}_{1}^{2}\end{array}$ \\
\hline
\end{tabular}


TABLE 3-Continued

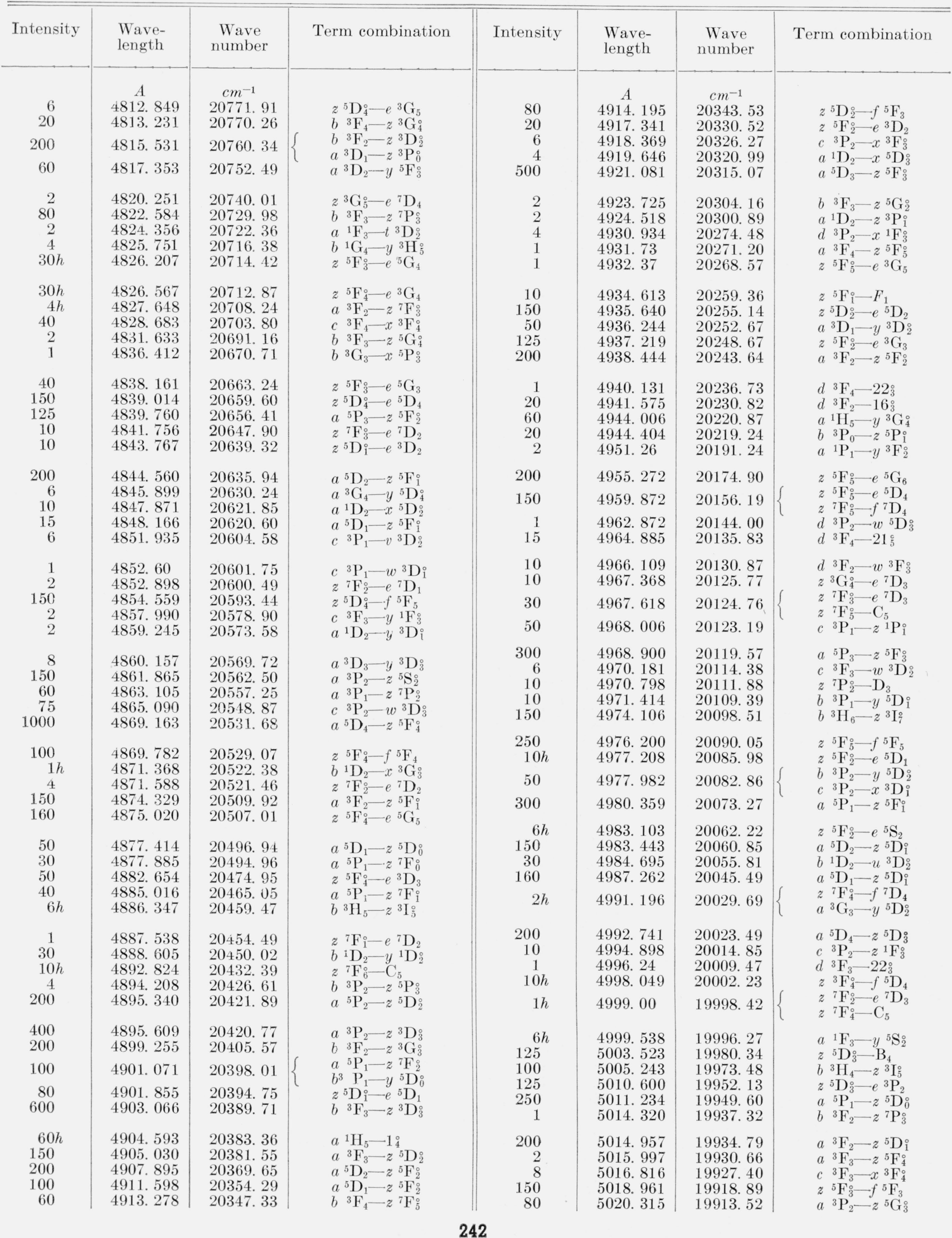


Table 3 -Continued

\begin{tabular}{|c|c|c|c|c|c|c|c|}
\hline Intensity & $\begin{array}{l}\text { Wave- } \\
\text { length }\end{array}$ & $\begin{array}{c}\text { Wave } \\
\text { number }\end{array}$ & Term combination & Intensity & $\begin{array}{l}\text { Wave- } \\
\text { length }\end{array}$ & $\begin{array}{c}\text { Wave } \\
\text { number }\end{array}$ & Term combination \\
\hline $\begin{array}{r}300 \\
200 \\
4 \\
1 \\
2\end{array}$ & \begin{tabular}{l}
\multicolumn{1}{c}{$A$} \\
5026.197 \\
5028.162 \\
5031.895 \\
5032.34 \\
5032.900
\end{tabular} & $\begin{array}{c}c m^{-1} \\
\text { 19890. } 21 \\
19882.44 \\
19867.69 \\
19865.93 \\
19863.72\end{array}$ & 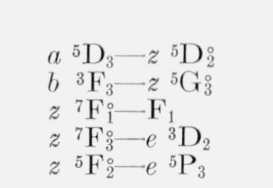 & $\begin{array}{r}400 \\
150 \\
50 \\
20 \\
20\end{array}$ & $\begin{array}{c}A \\
5155.140 \\
5160.008 \\
5168.062 \\
5168.636 \\
5170.078\end{array}$ & $\begin{array}{c}\mathrm{cm}^{-1} \\
19392.71 \\
19374.42 \\
19344.23 \\
19342.08 \\
19336.68\end{array}$ & $\begin{array}{l}a{ }^{5} \mathrm{D}_{1}-z^{5} \mathrm{D}_{2}^{\circ} \\
b{ }^{3} \mathrm{~F}_{4}-z^{3} \mathrm{G}_{5}^{\circ} \\
z^{3}{ }^{3} \mathrm{G}_{4}^{\circ}-e{ }^{7} \mathrm{D}_{4} \\
b^{3} \mathrm{H}_{4}-x^{3} \mathrm{~F}_{4}^{\circ} \\
c{ }^{3} \mathrm{~F}_{3}-z^{1} \mathrm{D}_{2}^{\circ}\end{array}$ \\
\hline $\begin{array}{r}6 \\
10 \\
150 \\
125 \\
160\end{array}$ & $\begin{array}{l}5036.986 \\
5039.037 \\
5039.627 \\
5040.357 \\
5040.738\end{array}$ & $\begin{array}{l}19847.61 \\
19839.53 \\
19837.21 \\
19834.33 \\
19832.83\end{array}$ & $\begin{array}{ll}b & { }^{3} \mathrm{G}_{3}-t^{3} \mathrm{D}_{2}^{\circ} \\
c & { }^{3} \mathrm{~F}_{4}-w^{3} \mathrm{D}_{3}^{\circ} \\
z & { }^{5} \mathrm{~F}_{3}^{\circ}-e^{3} \mathrm{G}_{4} \\
c & { }^{3} \mathrm{P}_{2}-x \\
a^{3} \mathrm{D}_{3}^{\circ} \\
{ }^{5} \mathrm{D}_{2}-z^{5} \mathrm{~F}_{3}^{\circ}\end{array}$ & $\begin{array}{r}1000 \\
50 \\
30 \\
6 \\
20\end{array}$ & $\begin{array}{l}5171.026 \\
5177.993 \\
5184.753 \\
5188.410 \\
5189.699\end{array}$ & $\begin{array}{l}\text { 19333. } 14 \\
19307.13 \\
19281.95 \\
19268.36 \\
19263.58\end{array}$ & 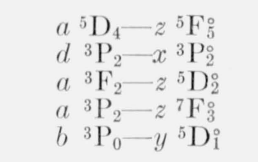 \\
\hline $\begin{array}{r}20 \\
20 \\
100 \\
200\end{array}$ & $\begin{array}{l}5041.350 \\
5041.978 \\
5045.432 \\
5047.308\end{array}$ & $\begin{array}{l}\text { 19830. } 43 \\
19827.96 \\
19814.38 \\
19807.02\end{array}$ & $\begin{array}{l}z^{5} \mathrm{~F}_{3}^{\circ}-e^{5} \mathrm{D}_{2} \\
b^{3} \mathrm{H}_{5}-x^{3} \mathrm{~F}_{4}^{\circ} \\
a^{3} \mathrm{D}_{2}-z^{5} \mathrm{P}_{2}^{\circ} \\
z^{5} \mathrm{C}_{6}^{\circ}-e{ }^{7} \mathrm{D}_{5} \\
a^{5} \mathrm{P}_{1}-z^{5} \mathrm{~F}_{2}^{\circ}\end{array}$ & $\begin{array}{c}4 \\
400 \\
50 \\
150 \\
100 h\end{array}$ & $\begin{array}{l}5193.026 \\
5195.017 \\
5197.058 \\
5199.873 \\
5202.127\end{array}$ & $\begin{array}{l}19251.23 \\
19243.86 \\
19236.30 \\
19225.88 \\
19217.55\end{array}$ & $\begin{array}{l}a^{3} \mathrm{D}_{1}-y{ }^{5} \mathrm{~F}_{2}^{\circ} \\
a{ }^{5} \mathrm{P}_{3}-z{ }^{5} \mathrm{~F}_{4}^{\circ} \\
b{ }^{3} \mathrm{~F}_{3}-z^{3} \mathrm{G}_{4}^{\circ} \\
z^{7} \mathrm{~F}_{6}^{\circ}-e^{7} \mathrm{D}_{5} \\
a^{1} \mathrm{H}_{5}-z^{3} \mathrm{H}_{4}^{\circ}\end{array}$ \\
\hline $\begin{array}{r}100 \\
1000 \\
10 \\
125\end{array}$ & $\begin{array}{l}5052.955 \\
5057.340 \\
5057.68 \\
5062.658\end{array}$ & $\begin{array}{l}19784.88 \\
19767.73 \\
19766.40 \\
19746.96\end{array}$ & 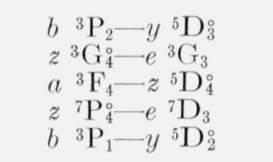 & $\begin{array}{c}4 \\
2 h \\
100 \\
100 \\
4\end{array}$ & $\begin{array}{l}5202.897 \\
5205.330 \\
5209.502 \\
5214.079 \\
5219.072\end{array}$ & $\begin{array}{l}19214.71 \\
19205.73 \\
19190.35 \\
19173.51 \\
19155.16\end{array}$ & $\begin{array}{lll}z & { }^{7} \mathrm{~F}_{4}^{\circ}-f & { }^{5} \mathrm{~F}_{3} \\
z^{3} & \mathrm{G}_{3}^{0}-f & { }^{7} \mathrm{D}_{2} \\
b & { }^{3} \mathrm{P}_{1}-z^{3} \mathrm{~F}_{2}^{\circ} \\
z & { }^{5} \mathrm{~F}_{4}^{\circ}-e^{5} \mathrm{D}_{3} \\
z^{7} & \mathrm{P}_{3}^{\circ}-e & { }^{7} \mathrm{D}_{2}\end{array}$ \\
\hline $\begin{array}{r}15 \\
10 \\
60 \\
250\end{array}$ & $\begin{array}{l}5063.196 \\
5066.668 \\
5071.013 \\
5072.993\end{array}$ & $\begin{array}{l}19744.87 \\
19731.34 \\
19714.43 \\
19706.74\end{array}$ & $\begin{array}{ll}d & { }^{3} \mathrm{~F}_{3}-19_{4}^{\circ} \\
d & { }^{3} \mathrm{~F}_{3}-w^{3} \mathrm{~F}_{2}^{\circ} \\
c & { }^{3} \mathrm{P}_{1}-x^{3} \mathrm{D}_{2}^{\circ} \\
a^{1} D_{2}-y{ }^{3} \mathbf{D}_{3}^{\circ} \\
a^{3}{ }^{3} \mathbf{F}_{2}-z^{5} \mathbf{F}_{3}^{\circ}\end{array}$ & $\begin{array}{c}300 \\
50 \\
2 h \\
40 \\
10\end{array}$ & $\begin{array}{l}5223.550 \\
5225.086 \\
5226.362 \\
5227.278 \\
5236.951\end{array}$ & $\begin{array}{l}19138.74 \\
19133.11 \\
19128.44 \\
19125.09 \\
19089.77\end{array}$ & $\begin{array}{l}a^{3} \mathrm{G}_{5}-z^{5} \mathrm{G}_{4}^{\circ} \\
z^{7} \mathrm{~F}_{4}^{\circ}-e^{3} \mathrm{G}_{4} \\
z^{3} \mathrm{D}_{3}^{\circ}-e{ }^{5} \mathrm{P}_{2} \\
c^{3} \mathrm{~F}_{4}-x^{3} \mathrm{D}_{3}^{\circ} \\
b^{3} \mathrm{~F}_{2}-z^{5} \mathrm{G}_{3}^{0}\end{array}$ \\
\hline $\begin{array}{c}4 h \\
100 \\
300 \\
125 \\
2 h\end{array}$ & $\begin{array}{l}5075.641 \\
5076.087 \\
5076.327 \\
5079.463 \\
5081.466\end{array}$ & $\begin{array}{l}19696.45 \\
19694.72 \\
19693.79 \\
19681.63 \\
19673.88\end{array}$ & $\begin{array}{lcl}z^{7} \mathrm{~F}_{4}^{\circ}-e & { }^{5} \mathrm{P}_{3} \\
a & { }^{5} \mathrm{P}_{3}-z^{5} \mathrm{D}_{2}^{\circ} \\
b & { }^{3} \mathrm{~F}_{3}-z^{3} \mathrm{~F}_{4}^{\circ} \\
z^{5} \mathrm{D}_{3}^{0}-e & { }^{5} \mathrm{D}_{3} \\
z^{7} & \mathrm{P}_{3}^{0}-f^{7} \mathrm{D}_{2}\end{array}$ & $\begin{array}{c}200 \\
150 \\
2 h \\
50\end{array}$ & $\begin{array}{l}5242.386 \\
5242.951 \\
5244.234 \\
5245.453\end{array}$ & $\begin{array}{l}19069.98 \\
19067.92 \\
19063.26 \\
19058.83\end{array}$ & $\begin{array}{l}a{ }^{3} \mathrm{P}_{2}-z^{5} \mathrm{~F}_{\mathrm{i}} \\
z^{5} \mathrm{~F}_{4}^{\circ}-e^{3} \mathrm{G}_{5} \\
z^{3} \mathrm{G}_{3}^{\circ}-e^{5} \mathrm{G}_{4} \\
c^{3} \mathrm{~F}_{3}-w^{3} \mathrm{D}_{3}^{\circ} \\
b^{3} \mathrm{H}_{5}-z^{3} \mathrm{I}_{b}^{\circ}\end{array}$ \\
\hline $\begin{array}{l}1 \\
2 h \\
1 h \\
150 H \\
100\end{array}$ & $\begin{array}{l}5082.91 \\
5083.992 \\
5084.58 \\
5086.776 \\
5090.086\end{array}$ & $\begin{array}{l}19668.29 \\
19664.10 \\
19661.83 \\
19653.34 \\
19640.56\end{array}$ & $\begin{array}{lll}z & { }^{3} \mathbf{F}_{4}^{\circ}-e & { }^{7} \mathrm{D}_{3} \\
c & { }^{3} \mathbf{F}_{2}-x & { }^{3} \mathbf{D}_{2}^{\circ} \\
z & { }^{7} \mathbf{F}_{3}^{\circ}-e & { }^{5} \mathrm{G}_{3} \\
z & { }^{5} \mathbf{F}_{3}^{\circ}- & { }^{5} \mathbf{F}_{4} \\
z & { }^{7} \mathbf{F}_{4}^{\circ}-e & { }^{7} \mathbf{D}_{4}\end{array}$ & $\begin{array}{l}2 h \\
10 \\
300 \\
200 \\
100\end{array}$ & $\begin{array}{l}5246.840 \\
5250.458 \\
5251.664 \\
5257.081 \\
5263.960\end{array}$ & $\begin{array}{l}19053.79 \\
19040.66 \\
19036.29 \\
19016.67 \\
18991.82\end{array}$ & $\begin{array}{l}z^{7} \mathrm{~F}_{5}^{\circ}-e^{5} \mathrm{G}_{5} \\
z^{7} \mathrm{P}_{4}^{\circ}-e^{5} \mathrm{P}_{3} \\
a^{3} \mathrm{G}_{4}-z^{3} \mathrm{G}_{3}^{\circ} \\
z^{5} \mathrm{G}_{3}^{\circ}-e^{5} \mathrm{G}_{3} \\
z^{3} \mathrm{G}_{5}^{\circ}-\mathrm{B}_{4}\end{array}$ \\
\hline $\begin{array}{l}600 \\
20 \\
10 h \\
200 \\
125\end{array}$ & $\begin{array}{l}5093.850 \\
5096.220 \\
5100.850 \\
5101.395 \\
5101.724\end{array}$ & $\begin{array}{l}\text { 19626. } 05 \\
19616.92 \\
19599.11 \\
19597.02 \\
19595.76\end{array}$ & $\begin{array}{ll}a & { }^{5} \mathbf{D}_{0}-z^{5} \mathbf{D}_{1}^{\circ} \\
c & { }^{3} \mathbf{F}_{4}-x^{3} \mathbf{F}_{3}^{\circ} \\
z & { }^{5} \mathbf{F}_{3}^{\circ}-e^{3} \mathbf{D}_{3} \\
b & { }^{3} \mathbf{F}_{2}-z^{3} \mathbf{D}_{3}^{\circ} \\
b & { }^{3} \mathbf{F}_{3}-z^{7} \mathbf{P}_{4}^{\circ}\end{array}$ & $\begin{array}{r}30 \\
100 \\
100 \\
2 \\
4\end{array}$ & $\begin{array}{l}5265.922 \\
5266.469 \\
5266.815 \\
5269.139 \\
5271.174\end{array}$ & $\begin{array}{l}\text { 18984. } 74 \\
18982.77 \\
18981.52 \\
18973.15 \\
18965.83\end{array}$ & $\begin{array}{lll}z & { }^{7} \mathrm{P}_{4}^{\circ}-e & { }^{7} \mathrm{D}_{4} \\
c & 3 \mathrm{P}_{2}-y & { }^{3} \mathrm{P}_{1} \\
a^{1} \mathrm{H}_{5}-z & { }^{1} \mathrm{G}_{4}^{\circ} \\
c^{1} \mathrm{H}^{3} \mathrm{~F}_{4}-z & { }^{1} \mathrm{H}_{5}^{\circ} \\
z^{7} \mathrm{P}_{3}^{0}-f^{5} \mathrm{D}_{4}\end{array}$ \\
\hline $\begin{array}{c}100 \\
200 \\
2 h \\
40 \\
60\end{array}$ & $\begin{array}{l}5106.570 \\
5107.071 \\
5111.249 \\
5114.590 \\
5114.996\end{array}$ & $\begin{array}{l}\text { 19577. } 16 \\
19575.24 \\
19559.24 \\
19546.46 \\
19544.91\end{array}$ & $\begin{array}{l}b^{3}{ }^{3} \mathrm{H}_{6}-x^{3} \mathrm{G}_{5}^{\circ} \\
a^{3} \mathrm{G}_{4}-z^{3} \mathbf{F}_{3}^{\circ} \\
b^{3}{ }^{3} \mathrm{G}_{4}-x^{1} \mathbf{F}_{3}^{\circ} \\
b^{1} \mathrm{G}_{4}-x^{3} \mathbf{G}_{4}^{\circ} \\
b^{1}{ }^{1} \mathrm{D}_{2}-y^{1} \mathbf{F}_{3}^{\circ}\end{array}$ & $\begin{array}{r}1 \\
1 \\
10 \\
60 \\
60\end{array}$ & $\begin{array}{l}5271.45 \\
5273.46 \\
5274.042 \\
5275.756 \\
5278.375\end{array}$ & $\begin{array}{l}18964.84 \\
18957.61 \\
18955.51 \\
18949.36 \\
18939.95\end{array}$ & $\begin{array}{ll}b^{3} \mathrm{G}_{4}-x^{5} \mathrm{~F}_{4} \\
z^{5} \mathrm{~F}_{4}-e{ }^{5} \mathrm{D}_{4} \\
z^{5} \mathrm{G}_{5}^{\circ}-e{ }^{7} \mathrm{D}_{4} \\
z^{7} \mathrm{~F}_{4}^{\circ}-f^{5} \mathrm{~F}_{4} \\
b^{3} \mathrm{~F}_{3}-z^{7} \mathrm{~F}_{4}\end{array}$ \\
\hline $\begin{array}{r}30 \\
125 \\
6 \\
150 \\
30\end{array}$ & $\begin{array}{l}5119.866 \\
5123.761 \\
5126.775 \\
5127.265 \\
5129.279\end{array}$ & $\begin{array}{l}19526.32 \\
19511.48 \\
19500.01 \\
19498.14 \\
19490.49\end{array}$ & $\begin{array}{ll}b & { }^{3} \mathrm{P}_{2}-z^{3} \mathrm{~F}_{2}^{\circ} \\
b & { }^{3} \mathrm{~F}_{2}-z^{5} \mathrm{G}_{2}^{\circ} \\
a & { }^{1} \mathrm{P}_{1}-y^{3} \mathrm{P}_{2}^{\circ} \\
a & { }^{5} \mathrm{P}_{1}-z^{5} \mathrm{D}_{1}^{\circ} \\
a^{3} & { }^{3} \mathrm{~F}_{4}-z^{7} \mathrm{D}_{3}^{\circ}\end{array}$ & $\begin{array}{l}20 h \\
150 \\
300 \\
50 \\
150\end{array}$ & $\begin{array}{l}5280.074 \\
5280.812 \\
5284.089 \\
5284.418 \\
5291.169\end{array}$ & $\begin{array}{l}\text { 18933. } 86 \\
18931.21 \\
18919.47 \\
18918.30 \\
18894.16\end{array}$ & $\begin{array}{lll}c & { }^{3} \mathrm{P}_{0}-y & { }^{3} \mathrm{~S}_{1} \\
a & { }^{5} \mathrm{D}_{3}-z & { }^{5} \mathrm{D}_{3}^{\circ} \\
a & { }^{3} \mathrm{~F}_{4}-z & { }^{7} \mathrm{D}_{4}^{\circ} \\
z & { }^{7} \mathrm{~F}_{5}^{\circ}-e & { }^{7} \mathrm{D}_{5} \\
b & { }^{3} \mathrm{~F}_{4}-z & { }^{5} \mathrm{~F}_{4}^{\circ}\end{array}$ \\
\hline $\begin{array}{r}20 \\
150 \\
1000 \\
40 \\
400\end{array}$ & $\begin{array}{l}5133.895 \\
5134.111 \\
5136.558 \\
5142.325 \\
5142.772\end{array}$ & $\begin{array}{l}19472.96 \\
19472.14 \\
19462.87 \\
19441.04 \\
19439.35\end{array}$ & $\begin{array}{l}a^{3} \mathrm{G}_{3}-z^{3} \mathrm{~F}_{2}^{\circ} \\
z^{5} \mathrm{~F}_{4}^{\circ}-\mathrm{B}_{4} \\
a{ }^{5} \mathrm{P}_{2}-z^{5} \mathrm{D}_{3}^{\circ} \\
b^{1} \mathrm{D}_{2}-w^{3} \mathrm{D}_{1}^{\circ} \\
a^{5}{ }^{5} \mathrm{D}_{3}-z^{5} \mathrm{~F}_{4}^{\circ}\end{array}$ & $\begin{array}{r}2 \\
2 \\
200 \\
50 \\
50\end{array}$ & $\begin{array}{l}\text { 5291. } 886 \\
5293.226 \\
5304.852 \\
5305.850 \\
5306.453\end{array}$ & $\begin{array}{l}18891.60 \\
18886.82 \\
18845.43 \\
18841.88 \\
18839.74\end{array}$ & 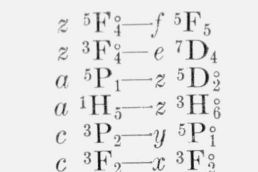 \\
\hline $\begin{array}{r}400 \\
10 \\
300 \\
125 \\
60\end{array}$ & $\begin{array}{l}5147.246 \\
5149.753 \\
5151.070 \\
5153.214 \\
5154.604\end{array}$ & $\begin{array}{l}19422.45 \\
19413.00 \\
19408.04 \\
19399.96 \\
19394.73\end{array}$ & $\begin{array}{l}a{ }^{3} \mathrm{~F}_{3}-z^{5} \mathrm{D}_{3}^{\circ} \\
c{ }^{3} \mathrm{~F}_{2}-z^{1} \mathrm{D}_{2}^{\circ} \\
a^{5} \mathrm{D}_{2}-z^{5} \mathrm{D}_{2}^{\circ} \\
a^{3} \mathrm{P}_{1}-z^{5} \mathrm{~S}_{2}^{\circ} \\
a^{3} \mathrm{P}_{2}-z^{7} \mathrm{~F}_{2}^{\circ} \\
a^{1} \mathrm{H}_{5}-y^{3} \mathrm{G}_{5}^{\circ}\end{array}$ & $\begin{array}{r}60 \\
100 u \\
200 \\
4 \\
1\end{array}$ & $\begin{array}{l}\text { 5307. } 284 \\
5309.259 \\
5315.339 \\
5316.589 \\
5317.92\end{array}$ & $\begin{array}{l}\text { 18836. } 79 \\
18829.78 \\
18808.24 \\
18803.82 \\
18799.12\end{array}$ & $\begin{array}{l}z^{3} \mathrm{G}_{4}^{\circ}-e^{3} \mathrm{G}_{4} \\
a^{5} \mathrm{D}_{4}-z^{5} \mathrm{D}_{1}^{\circ} \\
c^{3} \mathrm{P}_{2}-y^{3} \mathrm{P}_{2}^{\circ} \\
a^{3} \mathrm{P}_{2}-z^{5} \mathrm{~F}_{2}^{\circ} \\
z^{5} \mathrm{G}_{2}^{\circ}-e^{3} \mathrm{D}_{2}\end{array}$ \\
\hline
\end{tabular}


Table 3-Continued

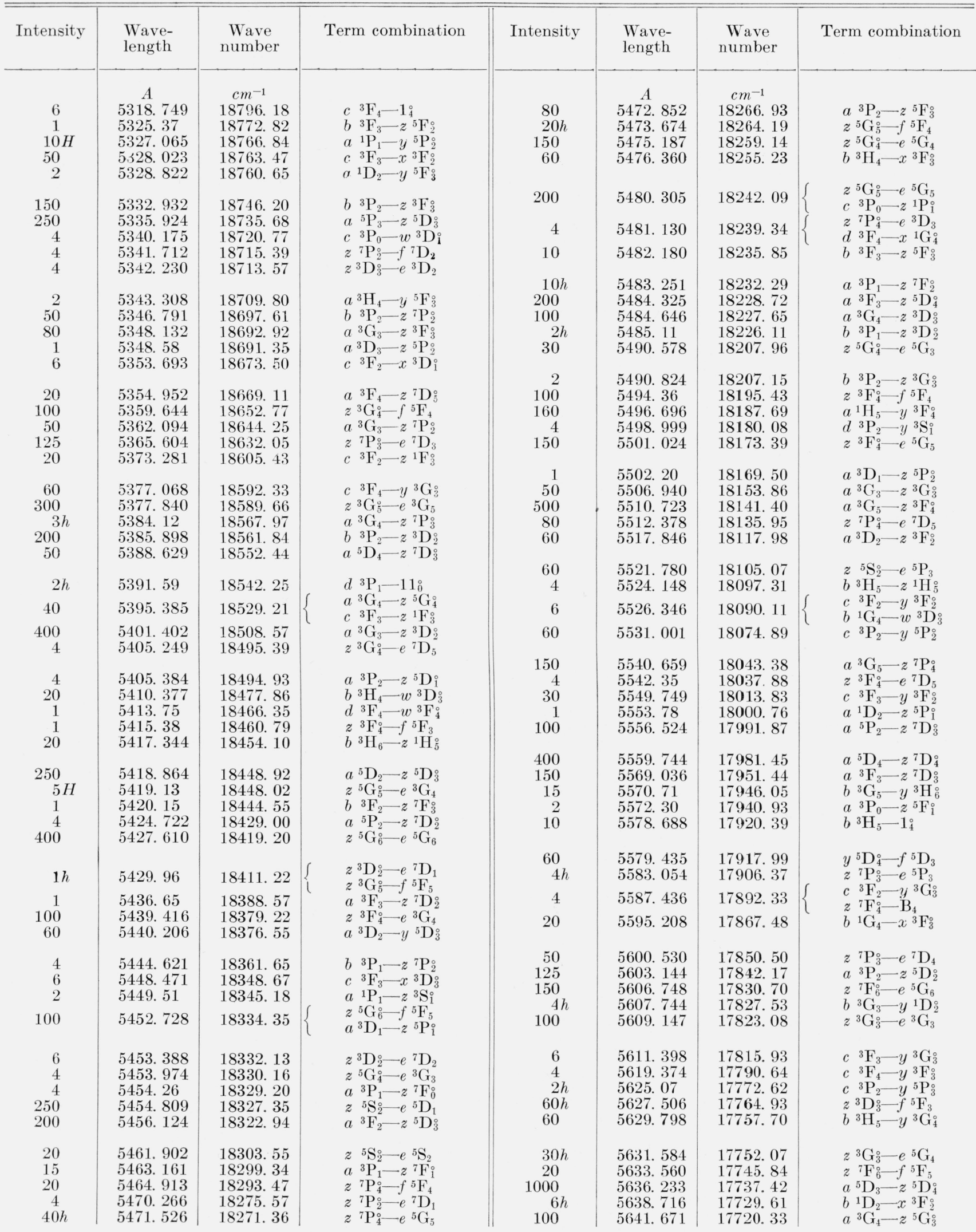


TABLE 3-Continued

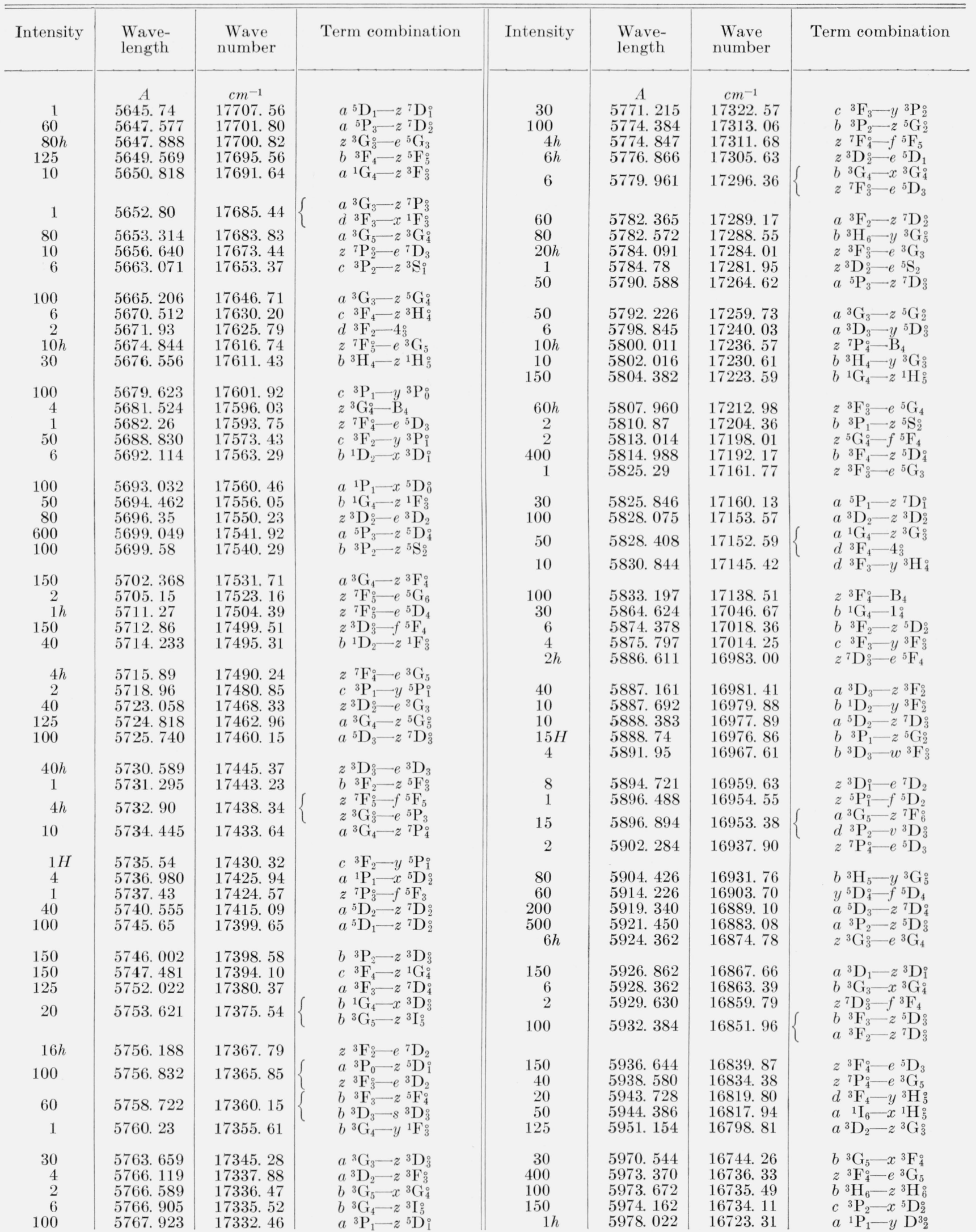


Table 3-Continued

\begin{tabular}{|c|c|c|c|c|c|c|c|}
\hline Intensity & $\begin{array}{l}\text { Wave- } \\
\text { length }\end{array}$ & $\begin{array}{c}\text { Wave } \\
\text { number }\end{array}$ & Term combination & Intensity & $\begin{array}{l}\text { Wave- } \\
\text { length }\end{array}$ & $\begin{array}{c}\text { Wave } \\
\text { number }\end{array}$ & Term combination \\
\hline $\begin{array}{c}10 \\
8 \\
2 h \\
200 \\
2 h\end{array}$ & $\begin{array}{c}A \\
5980.643 \\
5982.243 \\
5984.966 \\
5988.676 \\
5989.594\end{array}$ & $\begin{array}{c}c m^{-1} \\
16715.98 \\
16711.51 \\
16703.91 \\
16693.56 \\
16691.00\end{array}$ & $\begin{array}{l}c{ }^{3} \mathrm{P}_{1}-y{ }^{5} \mathrm{P}_{2}^{\circ} \\
z^{5} \mathrm{G}_{5}^{\circ}-e^{5} \mathrm{G}_{6} \\
b^{3} \mathrm{D}_{2}-9_{1}^{\circ} \\
a{ }^{5} \mathrm{P}_{3}-z^{7} \mathrm{D}_{4}^{\circ} \\
z^{3} \mathrm{G}_{3}^{\circ}-f{ }^{5} \mathrm{~F}_{4}\end{array}$ & $\begin{array}{l}30 h \\
50 \\
4 \\
2 H \\
4\end{array}$ & $\begin{array}{c}A \\
6210.31 \\
6210.75 \\
6213.63 \\
6217.35 \\
6217.52\end{array}$ & $\begin{array}{c}c m^{-1} \\
16097.80 \\
16096.66 \\
16089.20 \\
16079.57 \\
16079.13\end{array}$ & $\begin{array}{ll}z & { }^{3} \mathbf{F}_{3}^{\circ}-e{ }^{3} \mathrm{D}_{3} \\
b^{3} \mathrm{G}_{4}-x^{3} \mathrm{G}_{5}^{\circ} \\
d^{3} \mathrm{P}_{2}-x^{3} \mathrm{D}_{1}^{\circ} \\
z & { }^{5} \mathbf{F}_{3}^{\circ}-e^{3} \mathrm{~F}_{2} \\
z^{5} \mathbf{F}_{5}^{\circ} & f^{3} \mathbf{F}_{4}\end{array}$ \\
\hline $\begin{array}{r}3 \\
2 \\
20 \\
150 \\
4\end{array}$ & $\begin{array}{l}5991.194 \\
5991.485 \\
5992.580 \\
5993.658 \\
6002.889\end{array}$ & $\begin{array}{l}16686.54 \\
16685.73 \\
16682.68 \\
16679.68 \\
16654.03\end{array}$ & $\begin{array}{ll}d^{3}{ }^{3} \mathrm{~F}_{4}-x^{3} \mathrm{G}_{3}^{\circ} \\
c{ }^{3} \mathrm{P}_{2}-y{ }^{3} \mathrm{D}_{1}^{\circ} \\
a^{1}{ }^{1} \mathrm{D}_{2}-y{ }^{5} \mathrm{D}_{2}^{\circ} \\
a^{3}{ }^{3} \mathrm{P}_{1}-z z^{5} \mathrm{D}_{2}^{\circ} \\
c & { }^{3} \mathrm{~F}_{4}-z^{3} \mathrm{H}_{5}^{\circ}\end{array}$ & $\begin{array}{c}10 \\
300 \\
10 \\
20 \\
6 h\end{array}$ & $\begin{array}{l}6219.58 \\
6225.22 \\
6225.67 \\
6231.81 \\
6233.68\end{array}$ & $\begin{array}{l}16073.81 \\
16059.25 \\
16058.09 \\
16042.27 \\
16037.45\end{array}$ & $\begin{array}{l}a^{3} \mathrm{G}_{4}-z^{5} \mathrm{~F}_{3}^{\circ} \\
b^{3} \mathrm{~F}_{2}-z^{5} \mathrm{D}_{3}^{0} \\
b^{1} \mathrm{G}_{4}-y^{3} \mathrm{G}_{5}^{\circ} \\
z^{7} \mathrm{D}_{5}^{\circ}-e^{5} \mathrm{~F}_{5} \\
y^{5} \mathrm{~F}_{2}^{0}-f^{5} \mathbf{D}_{2}\end{array}$ \\
\hline $\begin{array}{r}30 \\
2 \\
6 \\
6\end{array}$ & $\begin{array}{l}6003.872 \\
6006.972 \\
6009.108 \\
6012.776\end{array}$ & $\begin{array}{l}16651.31 \\
16642.71 \\
16636.80 \\
16626.65\end{array}$ & $\begin{array}{l}a^{3} \mathrm{G}_{4}-z^{7} \mathrm{~F}_{5}^{\circ} \\
z^{5} \mathrm{G}_{3}^{\circ}-e{ }^{5} \mathrm{D}_{3} \\
z^{7} \mathrm{D}_{2}^{\circ}-e^{3} \mathrm{~F}_{3} \\
z^{3} \mathrm{G}_{3}^{\circ}-e^{3} \mathrm{D}_{3} \\
z^{5} \mathrm{G}_{5}^{\circ}-f^{5} \mathrm{~F}_{5}\end{array}$ & $\begin{array}{c}100 \\
10 h \\
10 \\
150 \\
6\end{array}$ & $\begin{array}{l}6235.63 \\
6235.97 \\
6241.62 \\
6252.067 \\
6255.293\end{array}$ & $\begin{array}{l}16032.44 \\
16031.56 \\
16017.05 \\
15990.29 \\
15982.04\end{array}$ & $\begin{array}{l}b^{3} \mathrm{H}_{4}-z{ }^{1} \mathrm{G}_{4}^{\circ} \\
c{ }^{3} \mathrm{P}_{2}-y{ }^{3} \mathrm{D}_{2}^{\circ} \\
a^{3} \mathrm{D}_{3}-z^{3} \mathrm{D}_{2}^{\circ} \\
a^{3} \mathrm{D}_{2}-z^{3} \mathrm{D}_{3}^{\circ} \\
a^{1}{ }^{1} \mathrm{~F}_{3}-x^{3} \mathrm{~F}_{2}^{\circ}\end{array}$ \\
\hline $\begin{array}{c}30 h \\
100 \\
4 \\
40 h \\
20\end{array}$ & $\begin{array}{l}6021.840 \\
6022.302 \\
6026.344 \\
6027.497 \\
6033.341\end{array}$ & $\begin{array}{l}16601.62 \\
16600.35 \\
16589.22 \\
16586.04 \\
16569.98\end{array}$ & $\begin{array}{l}z^{3} \mathrm{D}_{2}^{\circ}-f^{5} \mathrm{~F}_{3} \\
c^{3} \mathbf{F}_{4}-y{ }^{3} \mathbf{F}_{4}^{\circ} \\
c^{3} \mathbf{F}_{3}-y{ }^{5} \mathrm{P}_{2}^{\circ} \\
y^{5} \mathbf{D}_{3}^{\circ}-e{ }^{7} \mathrm{D}_{3} \\
y^{5} \mathrm{D}_{4}^{\circ}-e{ }^{7} \mathrm{D}_{3}\end{array}$ & $\begin{array}{r}6 \\
4 \\
20 \\
4\end{array}$ & $\begin{array}{l}6258.00 \\
6270.07 \\
6274.503 \\
6275.12\end{array}$ & $\begin{array}{l}15975.13 \\
15944.38 \\
15933.11 \\
15931.54\end{array}$ & $\begin{array}{l}b{ }^{3} \mathrm{G}_{5}-z^{3} \mathrm{I}_{6}^{\circ} \\
b^{3} \mathrm{D}_{3}-t^{3} \mathrm{D}_{2}^{\circ} \\
z{ }^{5} \mathrm{P}_{3}^{\circ}-e e^{7} \mathrm{D}_{3} \\
z^{3} \mathrm{D}_{1}^{\circ}-e{ }^{5} \mathrm{D}_{1} \\
b^{3} \mathrm{G}_{3}-x^{3} \mathrm{D}_{2}^{\circ}\end{array}$ \\
\hline $\begin{array}{c}4 h \\
4 \\
100 \\
20 \\
4 h\end{array}$ & $\begin{array}{l}6037.735 \\
6038.734 \\
6046.376 \\
6054.071 \\
6057.453\end{array}$ & $\begin{array}{l}16557.92 \\
16555.18 \\
16534.25 \\
16513.24 \\
16504.02\end{array}$ & $\begin{array}{l}z{ }^{3} \mathrm{~F}_{4}^{\circ}-f^{5} \mathrm{~F}_{5} \\
d^{3} \mathrm{P}_{2}-w^{3} \mathrm{D}_{3}^{\circ} \\
a^{1} \mathrm{D}_{2}-z^{3} \mathrm{D}_{1}^{\circ} \\
z^{3} \mathrm{D}_{2}-e^{5} \mathrm{D}_{2} \\
z^{3} \mathrm{~F}_{2}^{\circ}-e^{3} \mathrm{G}_{3}\end{array}$ & $\begin{array}{r}100 \\
6 \\
4 \\
50 \\
300\end{array}$ & $\begin{array}{l}6284.49 \\
6285.68 \\
6289.34 \\
6290.100 \\
6295.220\end{array}$ & $\begin{array}{l}15907.79 \\
15904.78 \\
15895.52 \\
15893.60 \\
15880.68\end{array}$ & $\begin{array}{l}c{ }^{3} \mathrm{P}_{1}-x^{5} \mathrm{D}_{1}^{\circ} \\
a^{3} \mathrm{D}_{2}-z^{5} \mathrm{G}_{2}^{\circ} \\
a^{3} \mathrm{G}_{3}-z^{7} \mathrm{~F}_{4}^{\circ} \\
y{ }^{5} \mathrm{~F}_{4}^{\circ}-f^{5} \mathrm{D}_{3} \\
b^{1} \mathrm{G}_{4}-z^{3} \mathrm{H}_{4}^{\circ}\end{array}$ \\
\hline $\begin{array}{l}20 \\
10 \\
1 h \\
2\end{array}$ & $\begin{array}{l}6065.189 \\
6073.90 \\
6077.79 \\
6078.831\end{array}$ & $\begin{array}{l}16482.97 \\
16459.33 \\
16448.79 \\
16445.98\end{array}$ & $\begin{array}{l}z^{5} \mathrm{P}_{2}-f^{5} \mathrm{D}_{3} \\
a^{3} \mathrm{D}_{1}-z^{3} \mathrm{~F}_{2}^{\circ} \\
d^{3} \mathrm{~F}_{3}-x^{3} \mathrm{G}_{3}^{\circ} \\
y^{5} \mathrm{D}_{1}^{\circ}-e{ }^{7} \mathrm{D}_{2} \\
b^{3} \mathrm{H}_{4}-y^{3} \mathrm{G}_{5}^{\circ}\end{array}$ & $\begin{array}{r}30 \\
40 \\
10 \\
2 \\
60\end{array}$ & $\begin{array}{l}\text { 6303. } 242 \\
6304.49 \\
6306.79 \\
6307.60 \\
6309.66\end{array}$ & $\begin{array}{l}15860.47 \\
15857.33 \\
15851.54 \\
15849.51 \\
15844.33\end{array}$ & $\begin{array}{l}y^{5} \mathrm{D}_{3}^{\circ}-e{ }^{5} \mathrm{P}_{3} \\
c{ }^{3} \mathrm{~F}_{2}-x x^{5} \mathrm{D}_{1}^{\circ} \\
z^{5} \mathrm{D}_{1}^{\circ}-e{ }^{3} \mathrm{~F}_{2} \\
b^{3} \mathrm{D}_{2}-x{ }^{1} \mathrm{~F}_{3}^{\circ} \\
y^{5} \mathrm{D}_{4}^{\circ}-e{ }^{5} \mathrm{P}_{3}\end{array}$ \\
\hline $\begin{array}{c}200 \\
60 \\
50 \\
10 \\
300 h\end{array}$ & $\begin{array}{l}6080.08 \\
6083.522 \\
6085.121 \\
6089.426 \\
6090.522\end{array}$ & $\begin{array}{l}16442.60 \\
16433.30 \\
16428.98 \\
16417.37 \\
16414.41\end{array}$ & $\begin{array}{l}z^{3} \mathrm{D}_{3}^{\circ}-\mathrm{B}_{4} \\
c^{3} \mathrm{P}_{2}-x^{5} \mathrm{D}_{3}^{\circ} \\
b^{3} \mathrm{H}_{4}-y^{3} \mathrm{~F}_{3}^{\circ} \\
z^{3} \mathrm{~F}_{3}^{\circ}-f^{5} \mathrm{~F}_{3} \\
z^{3} \mathrm{D}_{3}^{\circ}-e^{3} \mathrm{P}_{2}\end{array}$ & $\begin{array}{r}4 \\
50 \\
4 \\
60 \\
50\end{array}$ & $\begin{array}{l}6310.37 \\
6311.10 \\
6311.39 \\
6316.73 \\
6324.24\end{array}$ & $\begin{array}{l}15842.55 \\
15840.72 \\
15839.99 \\
15826.60 \\
15807.81\end{array}$ & $\begin{array}{l}z^{5} \mathrm{G}_{4}^{\circ}-e^{5} \mathrm{D}_{3} \\
d^{3} \mathrm{P}_{2}-x^{3} \mathrm{D}_{3}^{\circ} \\
b^{3} \mathrm{G}_{4}-w^{3} \mathrm{D}_{3}^{\circ} \\
c^{3} \mathrm{P}_{2}-y^{3} \mathrm{D}_{3}^{\circ} \\
a^{3}{ }^{3} \mathrm{G}_{5}-z^{5} \mathrm{~F}_{4}^{\circ}\end{array}$ \\
\hline $\begin{array}{r}15 \\
100 \\
300 \\
60\end{array}$ & $\begin{array}{l}6101.560 \\
6108.97 \\
6116.770 \\
6119.844\end{array}$ & $\begin{array}{l}\text { 16384. } 72 \\
16364.84 \\
16343.97 \\
16335.76\end{array}$ & $\begin{array}{l}a{ }^{1} \mathrm{D}_{2}-y{ }^{5} \mathrm{D}_{3}^{\circ} \\
a^{3} \mathrm{G}_{5}-z^{5} \mathrm{G}_{6}^{\circ} \\
a^{1} \mathrm{G}_{4}-z^{3} \mathrm{D}_{3}^{\circ} \\
b^{3} \mathrm{~F}_{4}-z^{7} \mathrm{D}_{4}^{\circ} \\
z^{3} \mathbf{F}_{3}^{\circ}-e^{3} \mathrm{G}_{4}\end{array}$ & $\begin{array}{r}150 \\
3 \\
30 \\
20 \\
20\end{array}$ & $\begin{array}{l}6330.62 \\
6331.52 \\
6336.10 \\
6343.07 \\
6351.90\end{array}$ & $\begin{array}{l}15791.88 \\
15789.63 \\
15778.22 \\
15760.88 \\
15738.97\end{array}$ & $\begin{array}{l}z^{7} \mathrm{D}_{4}^{\circ}-e{ }^{5} \mathrm{~F}_{5} \\
z^{7} \mathrm{D}_{3}^{\circ}-e e^{3} \mathrm{~F}_{4} \\
b^{3} \mathrm{H}_{5}-z^{3} \mathrm{H}_{5}^{\circ} \\
y^{5} \mathrm{D}_{2}^{\circ}-e{ }^{5} \mathrm{~S}_{2} \\
z^{5} \mathrm{G}_{4}^{5}-e^{3} \mathrm{G}_{5}\end{array}$ \\
\hline $\begin{array}{r}6 \\
50 \\
1 \\
60\end{array}$ & $\begin{array}{l}6121.032 \\
6122.376 \\
6137.77 \\
6141.527\end{array}$ & $\begin{array}{l}16332.59 \\
16329.01 \\
16288.05 \\
16278.09\end{array}$ & $\begin{array}{l}d^{3}{ }^{3} \mathrm{P}_{2}-x{ }^{3} \mathrm{~F}_{3}^{\circ} \\
z^{3} \mathrm{~F}_{3}^{\circ}-e{ }^{5} \mathrm{D}_{2} \\
a^{3} \mathrm{G}_{5}-z^{3} \mathrm{G}_{5}^{\circ} \\
y^{5} \mathrm{D}_{2}^{\circ}-e^{7} \mathrm{D}_{3} \\
z{ }^{5} \mathrm{P}_{3}^{\circ}-f^{5} \mathrm{D}_{4}\end{array}$ & $\begin{array}{r}15 \\
150 \\
100 \\
100\end{array}$ & $\begin{array}{l}6357.75 \\
6357.95 \\
6363.41 \\
6376.45\end{array}$ & $\begin{array}{l}15724.49 \\
15723.99 \\
15710.50 \\
15678.37\end{array}$ & $\begin{array}{l}b^{3} \mathrm{H}_{5}-y{ }^{3} \mathrm{~F}_{4}^{\circ} \\
c{ }^{3} \mathrm{~F}_{4}-x{ }^{5} \mathrm{D}_{3}^{\circ} \\
z^{5} \mathrm{D}_{2}^{\circ}-e^{5} \mathrm{~F}_{3} \\
a^{3} \mathrm{G}_{4}-z^{3} \mathrm{G}_{5}^{\circ}\end{array}$ \\
\hline $\begin{array}{r}10 \\
6 \\
200 \\
10 \\
50\end{array}$ & $\begin{array}{l}6150.073 \\
6170.237 \\
6170.605 \\
6173.127 \\
6174.210\end{array}$ & $\begin{array}{l}16255.47 \\
16202.35 \\
16201.38 \\
16194.76 \\
16191.92\end{array}$ & $\begin{array}{l}d^{3} \mathrm{P}_{2}-x^{3} \mathrm{~F}_{2}^{\circ} \\
z{ }^{5} \mathrm{~F}_{5}^{\circ}-e^{5} \mathrm{~F}_{4} \\
a^{3} \mathrm{D}_{3}-z^{3} \mathrm{~F}_{3}^{\circ} \\
y^{5} \mathrm{~F}_{3}^{\circ}-f^{5} \mathrm{D}_{2} \\
a^{3} \mathrm{G}_{3}-z^{3} \mathrm{G}_{4}^{\circ}\end{array}$ & $\begin{array}{r}60 \\
50 \\
15 \\
1 \\
80\end{array}$ & $\begin{array}{l}6382.98 \\
6384.66 \\
6388.06 \\
6388.79 \\
6390.21\end{array}$ & $\begin{array}{l}15662.33 \\
15658.21 \\
15649.88 \\
15648.09 \\
15644.61\end{array}$ & $\begin{array}{l}a^{3} \mathrm{D}_{3}-z^{3} \mathrm{G}_{3}^{\circ} \\
b{ }^{3} \mathrm{~F}_{3}-z^{5} \mathrm{D}^{\circ} \\
d^{3} \mathrm{~F}_{4}-x^{3} \mathrm{G}_{4} \\
a^{1}{ }^{1} \mathrm{G}_{4}-z^{3} \mathrm{~F}_{4}^{\circ} \\
b^{1} \mathrm{G}_{4}-z^{1} \mathrm{G}_{4}^{\circ}\end{array}$ \\
\hline $\begin{array}{r}100 \\
10 \\
4 \\
10 \\
10\end{array}$ & $\begin{array}{l}6176.774 \\
6177.736 \\
6179.973 \\
6185.835 \\
6189.488\end{array}$ & $\begin{array}{l}16185.20 \\
16182.68 \\
16176.82 \\
16161.49 \\
16151.96\end{array}$ & 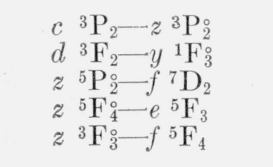 & $\begin{array}{r}2 \\
5 \\
2 \\
10 \\
2\end{array}$ & $\begin{array}{l}6395.89 \\
6401.40 \\
6404.86 \\
6406.07 \\
6413.33\end{array}$ & $\begin{array}{l}15630.72 \\
15617.27 \\
15608.83 \\
15605.88 \\
15588.22\end{array}$ & $\begin{array}{l}a^{3} \mathrm{D}_{1}-z^{7} \mathrm{P}_{2} \\
b^{3} \mathrm{G}_{4}-x^{3} \mathrm{~F}_{3}^{\circ} \\
z^{5} \mathrm{D}_{3}^{5}-e{ }^{3} \mathrm{~F}_{3} \\
z^{3} \mathrm{G}_{3}^{\circ}-e^{3} \mathrm{P}_{2} \\
b^{3} \mathrm{P}_{1}-z^{5} \mathrm{D}_{0}^{\circ}\end{array}$ \\
\hline $\begin{array}{r}300 \\
30 \\
20 \\
10 \\
400\end{array}$ & $\begin{array}{l}6192.552 \\
6195.33 \\
6195.97 \\
6197.15 \\
6199.42\end{array}$ & $\begin{array}{l}16143.96 \\
16136.73 \\
16135.06 \\
16131.99 \\
16126.08\end{array}$ & $\begin{array}{l}z^{3} \mathrm{D}_{3}^{\circ}-e{ }^{5} \mathrm{D}_{3} \\
b^{3} \mathrm{G}_{5}-x{ }^{3} \mathrm{G}_{5}^{\circ} \\
b^{3} \mathrm{H}_{6}-z z^{3} \mathrm{H}_{5}^{\circ} \\
a^{3} \mathrm{D}_{2}-z{ }^{5} \mathrm{~S}_{2}^{\circ} \\
a^{1} \mathrm{D}_{2}-z^{3} \mathrm{~F}_{2}^{\circ}\end{array}$ & $\begin{array}{r}100 \\
10 \\
6 \\
200 \\
4\end{array}$ & $\begin{array}{l}6417.56 \\
6429.55 \\
6432.10 \\
6444.84 \\
6445.85\end{array}$ & $\begin{array}{l}15577.94 \\
15548.89 \\
15542.73 \\
15512.00 \\
15509.57\end{array}$ & $\begin{array}{ll}y & { }^{5} \mathrm{~F}_{5}^{\circ}-f^{5} \mathrm{D}_{4} \\
z & 3 \mathrm{~F}_{2}^{\circ}-e^{5} \mathrm{D}_{2} \\
z^{5} \mathrm{~F}_{2}^{\circ}-e^{3} \mathrm{~F}_{2} \\
z^{5} \mathrm{D}_{3}^{5}-e^{5} \mathrm{~F}_{4} \\
c^{3} \mathrm{P}_{1}-x^{5} \mathrm{D}_{0}^{\circ}\end{array}$ \\
\hline
\end{tabular}


Table 3-Continued

\begin{tabular}{|c|c|c|c|c|c|c|c|}
\hline Intensity & $\begin{array}{l}\text { Wave- } \\
\text { length }\end{array}$ & $\begin{array}{c}\text { Wave } \\
\text { number }\end{array}$ & Term combination & Intensity & $\begin{array}{l}\text { Wave- } \\
\text { length }\end{array}$ & $\begin{array}{c}\text { Wave } \\
\text { number }\end{array}$ & Term combination \\
\hline $\begin{array}{r}4 \\
8 \\
10 \\
4 \\
8\end{array}$ & $\begin{array}{c}\text { A } \\
6451.88 \\
6456.91 \\
6457.43 \\
6461.19 \\
6472.51\end{array}$ & $\begin{array}{c}\mathrm{cm}^{-1} \\
15495.08 \\
15483.01 \\
15481.76 \\
15472.75 \\
15445.69\end{array}$ & $\begin{array}{ll}a^{3} \mathrm{D}_{1}-z{ }^{3} \mathrm{D}_{2}^{\circ} \\
a^{3} \mathrm{D}_{2}-z{ }^{5} \mathrm{G}_{3} \\
d^{3} \mathrm{~F}_{3}-y{ }^{1} \mathrm{~F}_{3}^{\circ} \\
b \quad{ }^{3} \mathrm{P}_{2}-z{ }^{5} \mathrm{D}_{1}^{\circ} \\
b{ }^{3} \mathrm{P}_{1}-z^{5} \mathrm{~F}_{2}^{\circ}\end{array}$ & $\begin{array}{r}2 \\
60 \\
1 \\
8 \\
1\end{array}$ & $\begin{array}{c}A \\
6779.15 \\
6787.20 \\
6792.94 \\
6805.53 \\
6807.00\end{array}$ & $\begin{array}{c}c m^{-1} \\
14747.04 \\
14729.55 \\
14717.10 \\
14689.88 \\
14686.71\end{array}$ & $\begin{array}{l}b{ }^{1} \mathrm{D}_{2}-x{ }^{5} \mathrm{D}_{1}^{\circ} \\
a^{3} \mathrm{H}_{6}-z{ }^{5} \mathrm{G}_{5}^{\circ} \\
a^{1} \mathrm{H}_{5}-y{ }^{5} \mathrm{~F}_{5}^{\circ} \\
a^{3} \mathrm{G}_{4}-z{ }^{5} \mathrm{D}_{3}^{\circ} \\
a^{3} \mathrm{P}_{1}-z^{7} \mathrm{D}_{2}^{\circ}\end{array}$ \\
\hline $\begin{array}{r}4 \\
12 \\
3 \\
100 \\
2\end{array}$ & $\begin{array}{l}6482.22 \\
6484.49 \\
6492.36 \\
6496.43 \\
6499.78\end{array}$ & $\begin{array}{l}15422.55 \\
15417.15 \\
15398.47 \\
15388.82 \\
15380.89\end{array}$ & 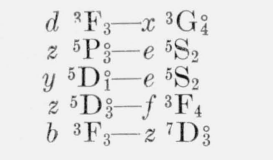 & $\begin{array}{r}8 \\
40 \\
3 \\
100 \\
150\end{array}$ & $\begin{array}{l}6812.90 \\
6813.52 \\
6821.73 \\
6823.88 \\
6824.17\end{array}$ & $\begin{array}{l}14673.99 \\
14672.65 \\
14654.99 \\
14650.38 \\
14649.75\end{array}$ & 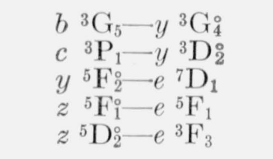 \\
\hline $\begin{array}{r}30 \\
2 \\
70 \\
15\end{array}$ & $\begin{array}{l}6519.06 \\
6531.60 \\
6540.24 \\
6544.23\end{array}$ & $\begin{array}{l}15335.40 \\
15305.96 \\
15285.74 \\
15276.42\end{array}$ & 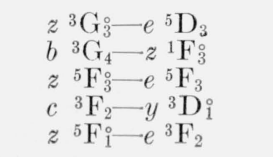 & $\begin{array}{r}20 \\
30 \\
1 \\
1 \\
3\end{array}$ & $\begin{array}{l}\text { 6831. } 44 \\
6831.54 \\
6832.71 \\
6834.11 \\
6837.04\end{array}$ & $\begin{array}{l}\text { 14634. } 17 \\
14633.95 \\
14631.45 \\
14628.45 \\
14622.18\end{array}$ & $\begin{array}{l}x^{5} \mathrm{D}_{3}^{\circ}-f^{5} \mathrm{D}_{2} \\
b{ }^{3} \mathrm{G}_{4}-y{ }^{3} \mathrm{G}_{4}^{\circ} \\
z{ }^{5} \mathrm{P}_{2}^{\circ}-e{ }^{5} \mathrm{D}_{1} \\
b^{3} \mathrm{D}_{3}-x{ }^{5} \mathrm{~F}_{4}^{\circ} \\
c^{3} \mathrm{~F}_{2}-y{ }^{3} \mathrm{D}_{2}^{\circ}\end{array}$ \\
\hline $\begin{array}{r}2 \\
2 \\
6 \\
60 \\
15\end{array}$ & $\begin{array}{l}6556.24 \\
6557.83 \\
6559.46 \\
6560.45 \\
6569.09\end{array}$ & $\begin{array}{l}15248.43 \\
15244.74 \\
15240.95 \\
15238.65 \\
15218.61\end{array}$ & $\begin{array}{l}c{ }^{3} \mathrm{~F}_{3}-x^{5} \mathrm{D}_{2}^{\circ} \\
b{ }^{3} \mathrm{P}_{2}-z{ }^{5} \mathrm{~F}_{3}^{\circ} \\
y^{3} \mathrm{D}_{3}^{\circ}-f^{5} \mathrm{D}_{2} \\
b^{3} \mathrm{H}_{4}-y^{3} \mathrm{~F}_{4}^{\circ} \\
z^{5} \mathrm{P}_{3}^{5}-e^{5} \mathrm{P}_{3}\end{array}$ & $\begin{array}{l}2 \\
1 \\
5 \\
1\end{array}$ & $\begin{array}{l}6843.13 \\
6843.83 \\
6850.84 \\
6852.93\end{array}$ & $\begin{array}{l}\text { 14609. } 17 \\
14607.67 \\
14592.72 \\
14588.27\end{array}$ & $\begin{array}{l}a^{3} \mathrm{G}_{5}-z^{5} \mathrm{~F}_{5}^{\circ} \\
z^{5} \mathrm{P}_{2}^{\circ}-e^{5} \mathrm{~S}_{2} \\
b^{5} \mathrm{G}_{4}-y^{3} \mathrm{G}_{3}^{\circ} \\
y^{3} \mathrm{G}_{5}^{\circ}-\mathrm{E}_{4} \\
b^{3} \mathrm{~F}_{2}-z^{7} \mathrm{D}_{3}^{\circ}\end{array}$ \\
\hline $\begin{array}{r}2 \\
30 \\
10 \\
10 \\
50\end{array}$ & $\begin{array}{l}6577.96 \\
6593.75 \\
6596.58 \\
6613.11 \\
6618.22\end{array}$ & $\begin{array}{l}15198.08 \\
15161.69 \\
15155.19 \\
15117.30 \\
15105.63\end{array}$ & $\begin{array}{l}a^{3} \mathrm{G}_{4}-z^{5} \mathrm{~F}_{4}^{\circ} \\
a^{1} \mathrm{D}_{2}-z^{3} \mathrm{D}_{2}^{\circ} \\
a^{3} \mathrm{D}_{3}-z^{5} \mathrm{G}_{4}^{\circ} \\
c^{3} \mathrm{~F}_{4}-y^{3} \mathrm{D}_{3}^{\circ} \\
a^{3} \mathrm{H}_{5}-z^{5} \mathrm{G}_{4}^{\circ}\end{array}$ & $\begin{array}{c}1 h \\
20 \\
1 \\
3 \\
1\end{array}$ & $\begin{array}{l}6858.76 \\
6872.92 \\
6875.15 \\
6883.51 \\
6891.70\end{array}$ & $\begin{array}{l}14575.87 \\
14545.84 \\
14541.13 \\
14523.47 \\
14506.21\end{array}$ & $\begin{array}{l}y{ }^{5} \mathrm{~F}_{2}^{\circ}-e{ }^{7} \mathrm{D}_{2} \\
c{ }^{3} \mathrm{~F}_{3}-y{ }^{3} \mathrm{D}_{2}^{\circ} \\
a \\
{ }^{1} \mathrm{~F}_{3}-y{ }^{3} \mathrm{P}_{2}^{\circ} \\
z^{3} \mathrm{G}_{5}^{\circ}-e{ }^{5} \mathrm{~F}_{4} \\
d^{3} \mathrm{P}_{2}-y{ }^{3} \mathrm{~F}_{3}^{\circ}\end{array}$ \\
\hline $\begin{array}{r}2 \\
3 \\
10 \\
6 \\
7\end{array}$ & $\begin{array}{l}6620.40 \\
6633.40 \\
6635.27 \\
6640.88 \\
6649.54\end{array}$ & $\begin{array}{l}15100.66 \\
15071.06 \\
15066.82 \\
15054.09 \\
15034.48\end{array}$ & $\begin{array}{ll}z & { }^{5} \mathrm{~F}_{4}^{\circ}-e^{3} \mathrm{~F}_{3} \\
z & \mathrm{P}_{2}^{\circ}-\mathrm{E}_{1} \\
z & { }^{3} \mathrm{~F}_{3}^{\circ}-e^{3} \mathrm{P}_{2} \\
c & { }^{3} \mathrm{P}_{1}-z^{3} \mathrm{P}_{1}^{\circ} \\
a^{1} & { }^{1} \mathrm{~F}_{3}-y{ }^{3} \mathrm{G}_{3}^{\circ}\end{array}$ & $\begin{array}{r}6 \\
1 \\
200 \\
1 \\
20\end{array}$ & $\begin{array}{l}\text { 6902. } 25 \\
6908.25 \\
6911.46 \\
6912.47 \\
6918.50\end{array}$ & $\begin{array}{l}14484.03 \\
14471.45 \\
14464.73 \\
14462.62 \\
14450.01\end{array}$ & $\begin{array}{c}b^{3} \mathrm{P}_{1}-z^{5} \mathrm{D}_{2}^{\circ} \\
z^{5} \mathrm{P}_{3}^{\circ}-f^{5} \mathrm{~F}_{4} \\
z^{5} \mathrm{~F}_{2}^{\circ}-e{ }^{5} \mathrm{~F}_{2} \\
y^{5} \mathrm{~F}_{5}^{\circ}-e{ }^{7} \mathrm{D}_{4} \\
d^{3} \mathrm{~F}_{4}-x^{3} \mathrm{G}_{5}^{\circ}\end{array}$ \\
\hline $\begin{array}{r}7 \\
2 \\
200 \\
10 \\
3\end{array}$ & $\begin{array}{l}6651.47 \\
6658.83 \\
6663.16 \\
6664.10 \\
6669.72\end{array}$ & $\begin{array}{l}15030.12 \\
15013.51 \\
15003.75 \\
15001.64 \\
14989.00\end{array}$ & $\begin{array}{l}c{ }^{3} \mathrm{P}_{2}-y{ }^{5} \mathrm{~F}_{2}^{\circ} \\
b^{3} \mathrm{G}_{5}-z^{1} \mathrm{H}_{5}^{\circ} \\
z^{5} \mathrm{~F}_{4}^{\circ}-e^{5} \mathrm{~F}_{4} \\
z^{5} \mathrm{~F}_{3}^{\circ}-e^{5} \mathrm{~F}_{2} \\
d^{3} \mathrm{P}_{2}-y^{3} \mathrm{P}_{1}^{0}\end{array}$ & $\begin{array}{r}2 \\
4 \\
800 \\
2 \\
1\end{array}$ & $\begin{array}{l}\text { 6920. } 13 \\
6921.97 \\
6923.21 \\
6926.67 \\
6934.20\end{array}$ & $\begin{array}{l}14446.61 \\
14442.77 \\
14440.18 \\
14432.97 \\
14417.30\end{array}$ & 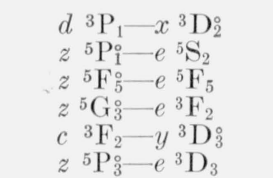 \\
\hline $\begin{array}{r}1 \\
2 \\
400 \\
20 \\
2\end{array}$ & $\begin{array}{l}6676.62 \\
6688.22 \\
6690.01 \\
6702.08 \\
6706.78\end{array}$ & $\begin{array}{l}14973.50 \\
14947.53 \\
14943.54 \\
14916.62 \\
14906.17\end{array}$ & $\begin{array}{l}b^{3} \mathrm{G}_{4}-z^{1} \mathrm{H}_{5}^{\circ} \\
c^{3} \mathrm{~F}_{3}-x^{5} \mathrm{D}_{3}^{5} \\
z^{5} \mathrm{D}_{4}^{\circ}-e e^{5} \mathrm{~F}_{5} \\
z^{5} \mathrm{~F}_{2}^{\circ}-e{ }^{5} \mathrm{~F}_{1} \\
z^{5} \mathrm{P}_{1}^{\circ}-\mathrm{F}_{1}\end{array}$ & $\begin{array}{l}3 \\
1 h \\
3 \\
3 \\
1\end{array}$ & $\begin{array}{l}\text { 6938. } 13 \\
6952.10 \\
6958.34 \\
6958.85 \\
6959.78\end{array}$ & $\begin{array}{l}\text { 14409. } 13 \\
14380.18 \\
14367.28 \\
14366.23 \\
14364.31\end{array}$ & $\begin{array}{l}z^{5} \mathrm{P}_{2}^{\circ}-e{ }^{5} \mathrm{P}_{3} \\
y^{5} \mathrm{~F}_{1}-e{ }^{7} \mathrm{D}_{1} \\
d^{3} \mathrm{~F}_{2}-x^{3} \mathrm{~F}_{2}^{\circ} \\
y^{5} \mathrm{~F}_{3}^{\circ}-e e^{5} \mathrm{P}_{2} \\
d^{3} \mathrm{~F}_{3}-v^{3} \mathrm{D}_{3}^{\circ}\end{array}$ \\
\hline $\begin{array}{r}30 \\
2 \\
80 \\
2 \\
7\end{array}$ & $\begin{array}{l}6707.56 \\
6717.57 \\
6718.31 \\
6718.91 \\
6721.81\end{array}$ & $\begin{array}{l}14904.44 \\
14882.23 \\
14880.59 \\
14879.26 \\
14872.84\end{array}$ & $\begin{array}{ll}b{ }^{1} \mathrm{G}_{4}-z^{3} \mathrm{H}_{5}^{\circ} \\
b^{3} \mathrm{D}_{2}-x^{3} \mathrm{P}_{2}^{\circ} \\
z z^{5} \mathrm{~F}_{4}^{\circ}-f^{3} \mathrm{~F}_{4} \\
y{ }^{5} \mathrm{~F}_{4}^{\circ}-f^{5} \mathrm{D}_{4} \\
c & { }^{3} \mathrm{P}_{2}-y{ }^{5} \mathrm{~F}_{3}^{\circ}\end{array}$ & $\begin{array}{r}20 \\
1 \\
2 \\
1 \\
300\end{array}$ & $\begin{array}{l}\text { 6960. } 76 \\
6963.06 \\
6968.43 \\
6971.12 \\
6981.98\end{array}$ & $\begin{array}{l}14362.29 \\
14357.54 \\
14346.48 \\
14340.94 \\
14318.64\end{array}$ & $\begin{array}{l}b^{3} \mathrm{H}_{4}-x^{5} \mathrm{D}_{3}^{\circ} \\
b^{3} \mathrm{G}_{3}-y^{3} \mathrm{~F}_{2}^{\circ} \\
a^{3} \mathrm{D}_{3}-z^{5} \mathrm{G}_{3}^{\circ} \\
c^{3} \mathrm{~F}_{3}-y^{3} \mathrm{D}_{3}^{\circ} \\
z^{5} \mathrm{D}_{3}^{\circ}-e^{3} \mathrm{~F}_{4}\end{array}$ \\
\hline $\begin{array}{r}1 \\
80 \\
20 \\
3 \\
2\end{array}$ & $\begin{array}{l}6724.87 \\
6730.46 \\
6731.79 \\
6733.95 \\
6738.12\end{array}$ & $\begin{array}{l}14866.07 \\
14853.72 \\
14850.79 \\
14846.03 \\
14836.84\end{array}$ & 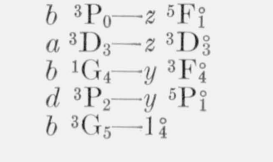 & $\begin{array}{l}3 \\
1 h \\
4 \\
1 \\
3\end{array}$ & $\begin{array}{l}\text { 6995. } 49 \\
6997.56 \\
6998.27 \\
7012.87 \\
7016.13\end{array}$ & $\begin{array}{l}14290.98 \\
14286.76 \\
14285.31 \\
14255.57 \\
14248.94\end{array}$ & $\begin{array}{l}b{ }^{3} \mathrm{P}_{0}-z^{5} \mathrm{D}_{1}^{\circ} \\
z^{3} \mathrm{~F}_{2}^{\circ}-e^{3} \mathrm{P}_{2} \\
z^{3} \mathrm{G}_{4}^{\circ}-e^{5} \mathrm{~F}_{3} \\
b^{3} \mathrm{D}_{3}-x^{3} \mathrm{P}_{2}^{\circ} \\
a^{3} \mathrm{H}_{4}-z^{5} \mathrm{G}_{4}^{\circ}\end{array}$ \\
\hline $\begin{array}{r}10 \\
2 \\
10 \\
100\end{array}$ & $\begin{array}{l}6742.92 \\
6750.39 \\
6751.73 \\
6756.54\end{array}$ & $\begin{array}{l}14826.28 \\
14809.87 \\
14806.93 \\
14796.39\end{array}$ & $\begin{array}{ll}c & { }^{3} \mathrm{P}_{1}-z^{3} \mathrm{P}_{2}^{\circ} \\
b & { }^{3} \mathrm{~F}_{3}-z^{7} \mathrm{D}_{4}^{\circ} \\
a^{1}{ }^{1} \mathrm{D}_{2}-z^{3} \mathrm{G}_{3}^{\circ} \\
z \\
z^{3} \mathrm{~F}_{3}^{\circ}-e^{5} \mathrm{D}_{3} \\
b^{3} \mathrm{G}_{4}-1_{4}^{\circ}\end{array}$ & $\begin{array}{c}4 \\
400 \\
3 \\
10 h \\
2\end{array}$ & $\begin{array}{l}7017.46 \\
7027.95 \\
7033.12 \\
7034.47 \\
7035.87\end{array}$ & $\begin{array}{l}\text { 14246. } 24 \\
14224.98 \\
14214.52 \\
14211.79 \\
14208.97\end{array}$ & $\begin{array}{l}a^{3} \mathrm{D}_{1}-z^{5} \mathrm{C}_{2} \\
z^{5} \mathrm{~F}_{3}^{\circ}-e^{3} \mathrm{~F}_{3} \\
b^{1} \mathrm{D}_{2}-x^{5} \mathrm{D}_{2} \\
y^{3} \mathrm{~F}_{4}^{\circ}-\mathrm{D}_{3} \\
y^{5} \mathrm{~F}_{2}^{\circ}-e^{5} \mathrm{P}_{2}\end{array}$ \\
\hline $\begin{array}{r}50 \\
150 \\
2 \\
100 \\
1 \mathrm{~h}\end{array}$ & $\begin{array}{l}6766.77 \\
6766.98 \\
6770.15 \\
6775.01 \\
6778.30\end{array}$ & $\begin{array}{l}14774.02 \\
14773.56 \\
14766.65 \\
14756.05 \\
14748.89\end{array}$ & $\begin{array}{l}z^{5} \mathrm{D}_{0}^{\circ}-e{ }^{5} \mathrm{~F}_{1} \\
z^{5} \mathrm{D}_{1}^{\circ}-e{ }^{5} \mathrm{~F}_{2} \\
a^{3} \mathrm{G}_{3}-z{ }^{5} \mathrm{D}_{2}^{\circ} \\
a^{3} \mathrm{H}_{4}-z^{3} \mathrm{G}_{3}^{\circ} \\
z^{5} \mathrm{~F}_{2}^{\circ}-e^{5} \mathrm{~F}_{3}\end{array}$ & $\begin{array}{r}2 \\
1 \\
9 \\
10 \\
60\end{array}$ & $\begin{array}{l}7039.85 \\
7042.49 \\
7057.12 \\
7058.42 \\
7060.36\end{array}$ & $\begin{array}{l}14200.93 \\
14195.61 \\
14166.18 \\
14163.57 \\
14159.68\end{array}$ & $\begin{array}{l}b^{3} \mathrm{~B}_{3}-y^{3} \mathrm{G}_{4}^{\circ} \\
d^{3} \mathrm{P}_{1}-z^{1} \mathrm{D}_{2}^{\circ} \\
b^{1} \mathrm{D}_{2}-y^{3} \mathrm{D}_{1}^{\circ} \\
c^{3}{ }^{3} \mathrm{~F}_{4}-y^{5} \mathrm{~F}_{3}^{\circ} \\
b^{3} \mathrm{G}_{3}-y^{3} \mathrm{G}_{3}^{\circ}\end{array}$ \\
\hline
\end{tabular}


TABLE 3-Continued

\begin{tabular}{|c|c|c|c|c|c|c|c|}
\hline Intensity & $\begin{array}{l}\text { Wave- } \\
\text { length }\end{array}$ & $\begin{array}{l}\text { Wave } \\
\text { number }\end{array}$ & Term combination & Intensity & $\begin{array}{l}\text { Wave- } \\
\text { length }\end{array}$ & $\begin{array}{l}\text { Wave } \\
\text { number }\end{array}$ & Term combination \\
\hline & $A$ & $\mathrm{~cm}^{-1}$ & & & $A$ & $\mathrm{~cm}^{-1}$ & \\
\hline $10 h$ & 7061. 12 & 14158. 16 & $z^{7} \mathbf{F}_{2}^{\circ}-e^{5} \mathbf{F}_{3}$ & 40 & 7348.72 & 13604.06 & $1_{4}^{\circ}-\mathrm{E}_{4}$ \\
\hline 100 & 7061.25 & 14157.90 & $a^{3} \mathrm{D}_{3}-z^{3} \mathrm{~F}_{4}^{\circ}$ & $2 h$ & 7356.27 & 13590.10 & $y^{3} \mathrm{D}_{3}^{\circ}-f^{5} \mathrm{D}_{4}$ \\
\hline 1 & 7061.80 & 14156. 79 & $b^{3} \mathrm{D}_{2}-u^{3} \mathrm{D}_{2}^{\circ}$ & 100 & 7377.77 & 13550.50 & $z^{7} \mathbf{F}_{5}^{0}-e^{5} \mathbf{F}_{4}$ \\
\hline 8 & 7070.15 & 14140.07 & $a^{1}{ }^{1} \mathrm{D}_{2}-z^{5} \mathrm{~S}_{2}^{\circ}$ & 40 & 7381.09 & 13544. 40 & $c{ }^{3} \mathrm{~F}_{3}-y{ }^{5} \mathrm{~F}_{2}^{\circ}$ \\
\hline 1 & 7073.70 & 14132.98 & $d^{3} \mathrm{~F}_{2}-z^{1} \mathrm{~F}_{3}^{\circ}$ & 2 & 7391. 33 & 13525.64 & $\begin{array}{l}y^{5} \mathbf{F}_{2}^{0}-e^{5} \mathbf{S}_{2} \\
y^{5} \mathrm{D}_{4}^{\circ}-e^{5} \mathrm{D}_{4}\end{array}$ \\
\hline 1 & 7076.16 & 14128. 06 & $z^{5} \mathrm{~F}_{3}^{\circ}-e^{5} \mathrm{~F}_{4}$ & & & & \\
\hline 8 & 7083.69 & 14113. 05 & $c{ }^{3} \mathrm{P}_{2}-z^{5} \mathrm{P}_{1}^{\circ}$ & 800 & 7393.93 & 13520.88 & $z^{7} \mathbf{F}_{4}^{\circ}-e^{3} \mathbf{F}_{3}$ \\
\hline 200 & 7086.04 & 14108. 37 & $a^{3} \mathrm{H}_{5}-z^{3} \mathbf{F}_{4}^{\circ}$ & 20 & 7407.51 & 13496. 10 & $a^{3} \mathrm{G}_{4}-z^{5} \mathrm{D}_{4}^{\circ}$ \\
\hline 200 & 7087.32 & 14105.82 & $a^{3} \mathrm{G}_{5}-z^{5} \mathrm{D}_{4}^{\circ}$ & 40 & 7410.27 & 13491.07 & $a^{1} \mathrm{D}_{2}-z^{5} \mathrm{G}_{3}^{4}$ \\
\hline 2 & 7104.15 & 14072. 40 & $a^{1} \mathbf{F}_{3}-z^{3} \mathbf{H}_{4}^{*}$ & 10 & 7419.59 & 13474. 12 & $x^{5} \mathrm{D}_{4}^{0}-e^{7} \mathrm{D}_{3}$ \\
\hline $20 h$ & 7110.57 & 14059. 69 & $a^{3} \mathrm{D}_{3}-z^{7} \mathrm{P}_{4}^{\circ}$ & 1 & 7425.44 & 13463.51 & $c{ }^{3} \mathbf{F}_{2}-y^{5} \mathbf{F}_{3}^{\circ}$ \\
\hline $1 h$ & 7120.43 & 14040. 23 & $y^{5} \mathrm{D}_{4}^{\circ}-\mathrm{B}_{4}$ & $1 h$ & 7427. 66 & 13459.48 & $y^{5} \mathrm{D}_{4}^{\circ}-f^{5} \mathrm{~F}_{5}$ \\
\hline 2 & 7120.74 & 14039. 61 & $a^{3} \mathrm{H}_{5}-z^{5} \mathrm{G}_{5}^{\circ}$ & 2 & 7429.55 & 13456. 06 & $d^{3} \mathrm{P}_{1}-x^{3} \mathrm{D}_{1}^{\circ}$ \\
\hline 15 & 7135.17 & 14011. 22 & $z^{5} \mathrm{G}_{2}^{\circ}-e^{3} \mathrm{~F}_{2}$ & 20 & 7438.32 & 13440.20 & $a^{3} \mathrm{H}_{4}-z^{5} \mathrm{G}_{3}^{0}$ \\
\hline 20 & 7135.64 & 14010.30 & $a^{3} \mathrm{H}_{5}-z^{7} \mathrm{P}_{4}^{\circ}$ & 20 & 7445.44 & 13427. 34 & $z^{7} \mathbf{F}_{5}^{\circ}-f^{3} \mathbf{F}_{4}$ \\
\hline 6 & 7140.81 & 14000.15 & $z^{7} \mathrm{~F}_{3}^{\circ}-e^{5} \mathrm{~F}_{2}$ & 10 & 7449.66 & 13419. 74 & $d^{3} \mathbf{F}_{2}-y^{3} \mathrm{G}_{3}^{\circ}$ \\
\hline 70 & 7141.71 & 13998. 39 & $a^{1}{ }^{1} \mathrm{D}_{2}-z{ }^{3} \mathrm{D}_{3}^{\circ}$ & $30 h$ & 7450.62 & 13418.01 & $z^{1} \mathrm{G}_{4}^{\circ}-\mathrm{D}_{3}$ \\
\hline 3 & 7146.41 & 13989. 18 & $y^{5} \mathrm{~F}_{2}^{\circ}-\mathrm{F}_{1}$ & 40 & 7453.62 & 13412.61 & $y^{3} \mathrm{D}_{3}^{\circ}-e^{5} \mathrm{P}_{2}$ \\
\hline $2 h$ & 7165.83 & 13951. 27 & $y{ }^{5} \mathrm{~F}_{3}^{\circ}-e^{3} \mathrm{D}_{2}$ & 2 & 7454.15 & 13411.65 & $a^{3} \mathrm{D}_{2}-z^{5} \mathrm{D}_{2}^{\circ}$ \\
\hline 15 & 7167.76 & 13947.52 & $a^{3} \mathrm{H}_{4}-z^{3} \mathrm{D}_{3}^{\circ}$ & 9 & 7458.43 & 13403. 96 & $a^{3} \mathrm{D}_{3}-z^{7} \mathrm{~F}_{4}^{\circ}$ \\
\hline 5 & 7168.46 & 13946.15 & $c{ }^{3} \mathrm{P}_{1}-y{ }^{5} \mathrm{~F}_{1}^{\circ}$ & 30 & 7463.70 & 13394. 49 & $b^{3} \mathrm{G}_{4}-z^{1} \mathrm{G}_{4}^{\circ}$ \\
\hline 2 & 7171.75 & 13939. 76 & $z^{3}{ }^{3} \mathrm{P}_{2}^{1}-f^{7}{ }^{7} \mathrm{D}_{2}$ & 5 & 7465.77 & 13390.78 & $x^{5} \mathrm{D}_{2}^{\circ}-f{ }^{7} \mathrm{D}_{2}$ \\
\hline $2 h$ & 7178. 94 & 13925.80 & $z^{7} \mathrm{P}_{4}^{\circ}-e^{5} \mathrm{~F}_{3}$ & 1 & 7467.78 & 13387.17 & $c^{3} \mathbf{F}_{3}-y^{5} \mathbf{F}_{3}^{0}$ \\
\hline 15 & 7182.20 & 13919.47 & $a^{1}{ }^{1} \mathrm{I}_{6}-y{ }^{1} \mathrm{H}_{5}^{\circ}$ & 700 & 7468.91 & 13385.15 & $z^{5} \mathrm{G}_{2}^{0}-e^{5} \mathrm{~F}_{1}$ \\
\hline 30 & 7183.01 & 13917. 91 & $a^{3} \mathrm{H}_{6}-z^{7} \mathrm{~F}_{5}^{\circ}$ & $\begin{array}{r}800 \\
30\end{array}$ & $\begin{array}{l}7485.75 \\
7486.07\end{array}$ & $\begin{array}{l}13355.04 \\
13354.47\end{array}$ & $\begin{array}{l}z^{5} \mathrm{G}_{3}^{\circ}-e{ }^{5} \mathbf{F}_{2} \\
a^{3} \mathrm{H}_{5}-z^{7} \mathbf{F}_{4}^{\circ}\end{array}$ \\
\hline 20 & 7194. 50 & 13895. 68 & $c{ }^{3} \mathrm{~F}_{2}-y{ }^{5} \mathbf{F}_{1}^{\circ}$ & & & & \\
\hline 2 & 7205.87 & 13873.75 & $z^{7} \mathrm{~F}_{2}^{\circ}-e^{5} \mathrm{~F}_{2}$ & 4 & 7495. 18 & 13338. 23 & $a^{1} \mathrm{P}_{1}-z^{3} \mathrm{D}_{1}^{\circ}$ \\
\hline 10 & 7212.57 & 13860.86 & $b{ }^{3} \mathrm{P}_{2}-z^{5} \mathrm{D}_{3}^{\circ}$ & 800 & 7499.74 & 13330.13 & $\begin{array}{c}y{ }^{3} \mathrm{~F}^{4}-{ }^{3} \mathrm{~F}_{3} \\
z^{3} \mathrm{G}_{5}-{ }^{3} \mathrm{~F}_{4}\end{array}$ \\
\hline 150 & 7219. 26 & 13848.02 & $b^{3} \mathrm{G}_{5}-y^{3} \mathrm{G}_{5}^{\circ}$ & $\begin{array}{r}800 \\
2\end{array}$ & $\begin{array}{l}499.84 \\
7516.05\end{array}$ & $\begin{array}{l}13330.13 \\
13301.20\end{array}$ & $\begin{array}{l}z^{0} \mathrm{G}_{5}^{5}-e^{0} \mathrm{H}^{4} \\
a^{1} \mathbf{I}_{6}-z^{3} \mathbf{I}_{5}^{\circ}\end{array}$ \\
\hline 5 & 7225.32 & 13836. 40 & $a^{3} \mathrm{D}_{2}-z^{5} \mathrm{~F}_{3}^{\circ}$ & 30 & 7519.59 & 13294. 94 & $b^{3} \mathrm{G}_{5}-z^{3} \mathrm{H}_{6}^{\circ}$ \\
\hline 4 & 7229.81 & 13827.81 & $z^{3} \mathrm{~F}_{4}^{\circ}-e^{5} \mathrm{~F}_{3}$ & 150 & 7532.07 & 13272.91 & $d^{3} \mathrm{P}_{2}-x{ }^{5} \mathrm{D}_{1}^{\circ}$ \\
\hline 4 & 7233. 96 & 13819. 88 & $y^{5} \mathrm{~F}_{4}^{\circ}-e^{5} \mathrm{P}_{3}$ & 7 & 7540.85 & 13257. 46 & $a^{3} \mathrm{G}_{5}-z^{7} \mathrm{D}_{4}^{\circ}$ \\
\hline 400 & 7238.90 & 13810. 45 & 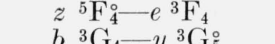 & 6 & 7544.19 & 13251.59 & $a^{3} \mathrm{H}_{4}-z^{3} \mathrm{~F}_{4}^{0}$ \\
\hline 20 & 7240.22 & 13807. 93 & $\begin{array}{l}b^{3} \mathrm{G}_{4}-y^{3} \mathrm{G}_{5}^{\circ} \\
y^{3} \mathrm{G}_{3}^{\circ}-\mathrm{E}_{4}\end{array}$ & 2 & 7549.87 & $\begin{array}{l}10201.09 \\
13241.62\end{array}$ & $\begin{array}{l}d{ }^{3} \mathrm{~F}_{3}-x^{3} \mathrm{D}_{3}^{0} \\
y^{5} \mathrm{~F}_{0}-\end{array}$ \\
\hline 3 & 7240.41 & 13807. 57 & $a^{3} \mathrm{G}_{3}-z^{5} \mathrm{D}_{3}^{\circ}$ & 700 & 7559.62 & 13224.54 & $z^{3} \mathrm{G}_{4}^{0}-e^{3} \mathrm{~F}_{3}$ \\
\hline 1 & 7247. 12 & 13794. 78 & $a^{1} \mathrm{G}_{4}-z^{3} \mathrm{G}_{5}^{3}$ & 2 & 7563.68 & 13217. 44 & $z^{5} \mathrm{G}_{2}^{\circ}-e^{5} \mathrm{~F}_{3}$ \\
\hline $1 h$ & 7247.54 & 13793. 98 & $y^{5} \mathrm{~F}_{2}^{\circ}-e^{3} \mathrm{D}_{2}$ & 5 & 7569.34 & 13207.56 & $y^{3} \mathrm{D}_{2}^{\circ}-e^{5} \mathrm{P}_{2}$ \\
\hline 10 & 7249.10 & 13791. 02 & $b^{3} \mathrm{G}_{4}-y^{3} \mathrm{~F}_{3}^{0}$ & 10 & 7575.08 & 13197.55 & $b^{3} \mathrm{G}_{3}-z^{3} \mathrm{H}_{4}^{\circ}$ \\
\hline 2 & 7255.16 & 13779. 50 & $y^{3} \mathrm{D}_{3}^{\circ}-e^{7} \mathrm{D}_{2}$ & 3 & 7598. 23 & 13157. 34 & $c{ }^{3} \mathrm{P}_{2}-y{ }^{5} \mathrm{D}_{1}^{\circ}$ \\
\hline 1 & 7255.47 & 13778. 91 & $d^{3} \mathrm{P}_{2}-y^{5} \mathrm{P}_{3}^{0}$ & 1 & 7600.39 & 13153. 60 & $a^{3} \mathrm{H}_{4}^{-}-z^{7} \mathrm{P}_{4}^{\circ}$ \\
\hline 10 & 7267.76 & 13755. 61 & $b^{3} \mathrm{H}_{4}-y^{3} \mathrm{D}_{3}^{\circ}$ & 4 & 7609.01 & 13138. 70 & $c{ }^{3} \mathrm{P}_{2}-z^{5} \mathrm{P}_{3}^{\circ}$ \\
\hline 1 & 7268.02 & 13755. 11 & $b^{3} \mathrm{D}_{2}-y^{3} \mathrm{~S}_{1}^{0}$ & 100 & 7612.97 & 13131.86 & $z^{3} \mathrm{D}_{3}^{\circ}-e^{5} \mathrm{~F}_{3}^{3}$ \\
\hline $4 h$ & 7272.24 & 13747. 13 & $y^{5} \mathrm{~F}_{3}^{\circ}-e^{5} \mathrm{G}_{3}$ & 500 & 7621.52 & 13117.13 & $z^{3} \mathrm{G}_{3}^{3}-e^{3} \mathrm{~F}_{2}^{3}$ \\
\hline $3 h$ & 7289.76 & 13714.10 & $y^{5} \mathrm{~F}_{1}^{\circ}-\mathrm{F}_{1}$ & $3 h$ & 7633.11 & 13097. 21 & $z^{7} \mathbf{F}_{2}^{\circ}-e^{3} \mathbf{F}_{3}^{2}$ \\
\hline & & & & 5 & 7647.41 & 13072.72 & $y^{5} \mathrm{~F}_{4}^{0}-f^{5} \mathrm{~F}_{4}$ \\
\hline $2 h$ & 7290. 90 & 13711.95 & $z{ }^{3} \mathrm{P}_{1}-f^{7} \mathrm{D}_{2}$ & 50 & 7658.43 & 13053. 91 & $z^{3} \mathrm{P}_{2}^{\circ}-e^{5} \mathrm{P}_{2}$ \\
\hline 20 & 7297. 10 & 13700. 30 & $a^{3} \mathrm{D}_{3}-z^{3} \mathrm{G}_{4}^{0}$ & 5 & 7660.35 & 13050.64 & $y^{5} \mathrm{~F}_{4}-e^{5} \mathrm{G}_{5}^{2}$ \\
\hline 2 & 7306. 36 & 13682. 94 & $y{ }^{5} \mathrm{~F}_{3}^{\circ}-e{ }^{5} \mathrm{~S}_{2}$ & 10 & 7665.14 & 13042. 49 & $a^{1} \mathbf{F}_{3}-y^{3} \mathrm{~F}_{4}^{\circ}$ \\
\hline 30 & 7312. 63 & 13671. 20 & $c{ }^{3} \mathrm{P}_{1}-y{ }^{5} \mathrm{~F}_{2}^{\circ}$ & $20 h$ & 7677.45 & 13021.58 & $y^{3} \mathrm{~F}_{3}^{\circ}-\mathrm{D}_{3}$ \\
\hline 20 & 7315.66 & 13665. 54 & $b^{1} \mathrm{D}_{2}-z^{3} \mathrm{P}_{2}^{\circ}$ & $20 h$ & 7687.50 & 13004.55 & $z^{3} \mathrm{G}_{4}^{\circ}-f^{3} \mathrm{~F}_{4}$ \\
\hline$\underline{4}$ & 7318. 81 & 13659. 66 & $d{ }^{3} \mathrm{P}_{2}-z^{3} \mathrm{~S}_{1}$ & & 7688.62 & 13002.66 & $y^{5} \mathrm{~F}_{3}^{\circ}-f^{5} \mathrm{~F}_{3}$ \\
\hline 7 & 7322.06 & 13653. 60 & $y^{3} \mathrm{D}_{2}^{0}-e^{7} \mathrm{D}_{1}$ & $\begin{array}{l}1 \\
3\end{array}$ & 7690.54 & 12999. 41 & $y^{3} \mathrm{D}_{1}-e^{7} \mathrm{D}_{1}$ \\
\hline 150 & 7323.57 & 13650.78 & $a^{3} \mathrm{H}_{5}-z^{3} \mathrm{G}_{4}^{\circ}$ & $2 h$ & 7691.61 & 12997. 60 & $y^{3} \mathrm{D}_{3}^{\circ}-e^{3} \mathrm{D}_{2}$ \\
\hline 3 & 7326. 21 & 13645. 86 & $b^{3} \mathrm{D}_{2}-y^{1} \mathbf{F}_{3}^{\circ}$ & 3 & 7694.52 & 12992. 69 & $y^{5} \mathrm{P}_{2}^{0}-f^{5} \mathrm{D}_{2}$ \\
\hline 10 & 7329.82 & 13639. 14 & $z^{5} \mathrm{G}_{3}^{0}-e^{5} \mathbf{F}_{3}$ & $4 h$ & 7697.42 & 12987. 79 & $y^{3} \mathrm{P}_{2}^{\circ}-f{ }^{7} \mathrm{D}_{1}$ \\
\hline 1 & 7334.43 & 13630.57 & $b{ }^{3} \mathrm{G}_{4}-z^{3} \mathrm{H}_{4}^{\circ}$ & 1 & 7697. 67 & 12987. 37 & $d^{3} \mathrm{~F}_{4}-y^{3} \mathrm{G}_{4}^{\circ}$ \\
\hline 10 & 7338.89 & 13622. 29 & $d^{3} \mathrm{P}_{1}-x^{3} \mathbf{F}_{2}^{\circ}$ & 9 & 7701.42 & 12981. 05 & $a^{3} \mathrm{D}_{1}-z^{5} \mathrm{~F}_{1}^{4}$ \\
\hline 1 & 7339.73 & 13620.73 & $c^{3}{ }^{3} \mathrm{~F}_{2}-y{ }^{5} \mathrm{~F}_{2}^{\circ}$ & 10 & 7719. 26 & 12951.05 & $x^{5} \mathrm{D}_{2}^{\circ}-e^{7} \mathrm{D}_{1}^{\circ}$ \\
\hline 9 & 7341. 44 & 13617.55 & $d^{3} \mathrm{~F}_{2}-y^{3} \mathrm{~F}_{2}^{\circ}$ & 500 & 7722.89 & 12944. 96 & $a^{3} \mathrm{H}_{6}-z^{3} \mathrm{G}_{5}^{\circ}$ \\
\hline 1 & 7343.49 & 13613. 75 & $y^{5} \mathrm{~F}_{5}^{\circ}-e^{7} \mathrm{D}_{5}$ & 3 & 7728.99 & 12934. 74 & $z^{5} \mathrm{~F}_{3}^{\circ}-e^{3} \mathrm{~F}_{4}^{0}$ \\
\hline
\end{tabular}


TABle 3 -Continued

\begin{tabular}{|c|c|c|c|c|c|c|c|}
\hline Intensity & $\begin{array}{l}\text { Wave- } \\
\text { length }\end{array}$ & $\begin{array}{c}\text { Wave } \\
\text { number }\end{array}$ & Term combination & Intensity & $\begin{array}{l}\text { Wave- } \\
\text { length }\end{array}$ & $\begin{array}{c}\text { Wave } \\
\text { number }\end{array}$ & Term combination \\
\hline $\begin{array}{c}20 \\
2 \\
1 \\
2 h \\
4\end{array}$ & $\begin{array}{c}A \\
7729.87 \\
7733.99 \\
7736.19 \\
7737.17 \\
7739.79\end{array}$ & $\begin{array}{c}c m^{-1} \\
12933.27 \\
12926.38 \\
12922.70 \\
12921.07 \\
12916.69\end{array}$ & $\begin{array}{ll}z & { }^{5} \mathrm{G}_{2}^{\circ}-e^{5} \mathrm{~F}_{2} \\
d & { }^{3} \mathrm{~F}_{2}-y^{3} \mathrm{P}_{2}^{\circ} \\
d & { }^{3} \mathrm{~F}_{3}-1_{4}^{\circ} \\
y & { }^{5} \mathrm{~F}_{3}^{\circ}-e^{3} \mathrm{G}_{4} \\
d & { }^{3} \mathrm{~F}_{3}-y^{3} \mathrm{~F}_{2}^{\circ}\end{array}$ & $\begin{array}{c}10 \\
2 h \\
7 \\
20 \\
2\end{array}$ & $\begin{array}{c}\text { A } \\
\text { 8074. } 43 \\
\text { 8083. } 23 \\
\text { 8089. } 47 \\
\text { 8090. } 23 \\
\text { 8091. } 05\end{array}$ & $\begin{array}{c}\mathrm{cm}^{-1} \\
12381.37 \\
12367.89 \\
12358.35 \\
12357.19 \\
12355.94\end{array}$ & $\begin{array}{l}a{ }^{1} \mathrm{D}_{2}-z^{5} \mathrm{~F}_{2}^{\circ} \\
b^{3} \mathrm{D}_{3}-x^{3} \mathbf{F}_{4}^{\circ} \\
z^{5} \mathrm{G}_{3}^{\circ}-f^{3} \mathbf{F}_{4} \\
z^{7} \mathbf{F}_{5}^{\circ}-e^{3} \mathbf{F}_{4} \\
d^{3} \mathrm{P}_{1}-y^{3} \mathrm{P}_{1}^{\circ}\end{array}$ \\
\hline $\begin{array}{l}7 \\
2 \\
6 \\
3 h \\
2\end{array}$ & $\begin{array}{l}7770.86 \\
7774.98 \\
7781.34 \\
7788.90 \\
7789.58\end{array}$ & $\begin{array}{l}12865.05 \\
12858.23 \\
12847.72 \\
12835.25 \\
12834.13\end{array}$ & $\begin{array}{l}z{ }^{7} \mathrm{P}_{4}^{\circ}-e^{3} \mathrm{~F}_{3} \\
x{ }^{5} \mathrm{D}_{1}^{\circ}-f^{7} \mathrm{D}_{2} \\
z^{3} \mathrm{D}_{3}^{\circ}-e^{5} \mathbf{F}_{2} \\
y^{3} \mathrm{P}_{0}^{\circ}-f^{7} \mathrm{D}_{1} \\
z^{3} \mathrm{P}_{2}^{\circ}-\mathrm{F}_{1}\end{array}$ & $\begin{array}{r}4 \\
150 \\
1 \\
15 \\
20\end{array}$ & $\begin{array}{l}8105.47 \\
8112.47 \\
8144.33 \\
8157.69 \\
8168.85\end{array}$ & $\begin{array}{l}\text { 12333. } 96 \\
12323.31 \\
12275.11 \\
12255.00 \\
12238.26\end{array}$ & $\begin{array}{l}d^{3} \mathrm{P}_{1}-y{ }^{3} \mathrm{P}_{0} \\
z^{3} \mathrm{G}_{3}^{\circ}-e^{5} \mathrm{~F}_{3} \\
a^{3} \mathrm{D}_{3}-z{ }^{5} \mathrm{D}_{2} \\
a^{3} \mathrm{H}_{5}-z^{3} \mathrm{G}_{5}^{\circ} \\
c^{3} \mathrm{P}_{2}-z^{3} \mathrm{~F}_{2}^{\circ}\end{array}$ \\
\hline $\begin{array}{c}300 \\
3 \\
1 h \\
2 \\
100\end{array}$ & $\begin{array}{l}7791.81 \\
7800.16 \\
7802.32 \\
7806.52 \\
7806.78\end{array}$ & $\begin{array}{l}12830.46 \\
12816.72 \\
12813.17 \\
12806.28 \\
12805.85\end{array}$ & $\begin{array}{l}z^{5} \mathrm{G}_{4}^{\circ}-e e^{5} \mathrm{~F}_{3} \\
x^{5} \mathrm{D}_{0}^{\circ}-e{ }^{7} \mathrm{D}_{1} \\
y^{3} \mathrm{P}_{1}^{\circ}-f^{7} \mathrm{D}_{1} \\
a^{1} \mathrm{G}_{4}-z{ }^{5} \mathrm{D}_{3}^{\circ} \\
x^{5} \mathrm{D}_{3}^{\circ}-e^{5} \mathrm{P}_{2}\end{array}$ & $\begin{array}{l}15 \\
30 h \\
2 \\
8 h \\
5\end{array}$ & $\begin{array}{l}8173.91 \\
8181.99 \\
8185.82 \\
8194.54 \\
8216.25\end{array}$ & $\begin{array}{l}12230.69 \\
12218.61 \\
12212.89 \\
12199.89 \\
12167.66\end{array}$ & $\begin{array}{l}z{ }^{7} \mathrm{~F}_{4}^{\circ}-e{ }^{3} \mathbf{F}_{4} \\
y{ }^{5} \mathrm{~F}_{5}^{\circ}-e{ }^{5} \mathrm{G}_{6} \\
d^{3} \mathrm{P}_{1}-y{ }^{5} \mathrm{P}_{1} \\
y^{5} \mathrm{~F}_{5}^{\circ}-e{ }^{5} \mathrm{D}_{4} \\
b^{3} \mathrm{G}_{3}-y{ }^{3} \mathbf{F}_{4}^{\circ}\end{array}$ \\
\hline $\begin{array}{c}5 \\
40 \\
5 \\
5 h\end{array}$ & $\begin{array}{l}7809.19 \\
7813.43 \\
7813.96 \\
7815.38\end{array}$ & $\begin{array}{l}12801.90 \\
12794.96 \\
12794.09 \\
12791.76\end{array}$ & $\begin{array}{l}b^{3} \mathrm{H}_{4}-y^{5} \mathrm{~F}_{3}^{\circ} \\
c^{3} \mathrm{P}_{2}-y{ }^{5} \mathrm{D}_{2}^{\circ} \\
a^{3} \mathrm{H}_{4}-z^{7} \mathrm{~F}_{3}^{\circ} \\
a^{3} \mathrm{H}_{4}-z^{3} \mathrm{G}_{4}^{\circ} \\
z^{7} \mathrm{P}_{3}^{\circ}-e^{5} \mathrm{~F}_{3}\end{array}$ & $\begin{array}{c}50 \\
2 \\
4 \\
15 h \\
4\end{array}$ & $\begin{array}{l}8220.48 \\
8231.96 \\
8237.46 \\
8239.21 \\
8246.80\end{array}$ & $\begin{array}{l}12161.40 \\
12144.44 \\
12136.33 \\
12133.75 \\
12122.58\end{array}$ & $\begin{array}{l}d^{3} \mathbf{F}_{4}-y^{3} \mathbf{G}_{5}^{\circ} \\
d^{3} \mathbf{F}_{4}-y^{3} \mathbf{F}_{3}^{\circ} \\
z^{3} \mathbf{D}_{2}^{\circ}-e{ }^{5} \mathbf{F}_{1} \\
y^{5} \mathbf{F}_{5}^{\circ}-f^{5} \mathbf{F}_{5} \\
x^{5} \mathbf{D}_{3}^{\circ}-e^{5} \mathbf{S}_{2}\end{array}$ \\
\hline $\begin{array}{r}70 \\
10 \\
100 \\
1 \\
40\end{array}$ & $\begin{array}{l}7829.81 \\
7830.51 \\
7833.37 \\
7834.02 \\
7834.78\end{array}$ & $\begin{array}{l}12768.19 \\
12767.05 \\
12762.39 \\
12761.33 \\
12760.09\end{array}$ & $\begin{array}{l}z^{7} \mathrm{P}_{4}^{\circ}-e^{5} \mathbf{F}_{4} \\
z^{3} \mathbf{F}_{4}^{\circ}-e^{3} \mathbf{F}_{3} \\
z^{3} \mathrm{D}_{2}^{\circ}-e^{3} \mathbf{F}_{2} \\
z^{3} \mathbf{G}_{5}^{\circ}-e{ }^{5} \mathbf{F}_{5} \\
d^{3} \mathbf{F}_{3}-y^{3} \mathbf{G}_{4}\end{array}$ & $\begin{array}{c}3 \\
400 \\
3 h \\
2 \\
30\end{array}$ & $\begin{array}{l}8251.32 \\
8264.95 \\
8269.02 \\
8281.00 \\
8281.98\end{array}$ & $\begin{array}{l}\text { 12115. } 94 \\
12095.96 \\
12090.01 \\
12072.52 \\
12071.09\end{array}$ & $\begin{array}{l}a^{1} \mathrm{G}_{4}-z^{5} \mathrm{~F}_{5}^{\circ} \\
z^{7} \mathrm{~F}_{6}^{0}-e^{5} \mathrm{~F}_{5} \\
x^{5} \mathrm{D}_{2}^{\circ}-e^{3} \mathrm{D}_{2} \\
a^{1} \mathrm{D}_{2}-z^{5} \mathrm{D}_{1}^{\circ} \\
z^{3} \mathrm{D}_{3}^{\circ}-e^{3} \mathrm{~F}_{3}\end{array}$ \\
\hline $\begin{array}{r}3 \\
100 \\
300 \\
20 \\
1\end{array}$ & $\begin{array}{l}7838.42 \\
7841.92 \\
7847.81 \\
7853.76 \\
7862.70\end{array}$ & $\begin{array}{l}12754.17 \\
12748.47 \\
12738.90 \\
12729.25 \\
12714.78\end{array}$ & $\begin{array}{l}c{ }^{3} \mathrm{P}_{1}-z^{5} \mathrm{P}_{1} \\
x^{5} \mathrm{D}_{4}^{\circ}-e^{5} \mathrm{P}_{3} \\
z^{5} \mathrm{G}_{5}^{\circ}-e^{5} \mathrm{~F}_{4} \\
y^{3} \mathrm{D}_{3}^{\circ}-e^{5} \mathrm{~S}_{2} \\
a^{3} \mathrm{D}_{1}-z^{5} \mathrm{~F}_{2}^{\circ}\end{array}$ & $\begin{array}{l}2 \\
2 \\
2 \\
2 \\
2 h\end{array}$ & $\begin{array}{l}8296.45 \\
8297.23 \\
8304.90 \\
8309.04 \\
8320.13\end{array}$ & $\begin{array}{l}\text { 12050. } 04 \\
12048.90 \\
12037.78 \\
12031.78 \\
12015.74\end{array}$ & $\begin{array}{l}y{ }^{5} \mathrm{P}_{2}-f{ }^{7} \mathrm{D}_{2} \\
y^{3} \mathrm{D}_{3}^{\circ}-f^{5} \mathrm{~F}_{3} \\
d{ }^{3} \mathrm{P}_{2}-y^{3} \mathrm{D}_{2}^{\circ} \\
z{ }^{3} \mathrm{~S}_{1}^{\circ}-e^{7} \mathrm{D}_{1} \\
y^{5} \mathrm{~F}_{4}^{\circ}-\mathrm{B}_{4}\end{array}$ \\
\hline $\begin{array}{r}10 \\
3 \\
10 \\
200 \\
400\end{array}$ & $\begin{array}{l}7871.92 \\
7875.27 \\
7876.78 \\
7881.47 \\
7890.40\end{array}$ & $\begin{array}{l}12699.89 \\
12694.49 \\
12692.05 \\
12684.50 \\
12670.14\end{array}$ & $\begin{array}{l}a^{3} \mathrm{D}_{3}-z^{5} \mathrm{~F}_{3}^{\circ} \\
b^{3} \mathrm{G}_{5}-z^{3} \mathrm{H}_{5}^{\circ} \\
d^{3} \mathrm{P}_{2}-y^{3} \mathrm{D}_{1}^{\circ} \\
z^{5} \mathrm{G}_{6}^{\circ}-e^{5} \mathrm{~F}_{5} \\
z^{3} \mathrm{~F}_{4}^{\circ}-e^{5} \mathbf{F}_{4}\end{array}$ & $\begin{array}{r}1 \\
250 \\
7 \\
200 \\
1\end{array}$ & $\begin{array}{l}8330.16 \\
8348.99 \\
8350.23 \\
8352.93 \\
8354.92\end{array}$ & $\begin{array}{l}\text { 12001. } 27 \\
11974.21 \\
11972.43 \\
11968.56 \\
11965.71\end{array}$ & $\begin{array}{l}x^{5} \mathrm{D}_{4}-f^{5} \mathrm{~F}_{4} \\
z^{3} \mathrm{D}_{3}-e e^{5} \mathrm{~F}_{4} \\
x^{5} \mathrm{D}_{1}-e e^{5} \mathrm{P}_{2} \\
z^{3} \mathrm{D}_{2}^{\circ}-e{ }^{5} \mathrm{~F}_{3} \\
a^{1} \mathrm{P}_{1}-z^{3} \mathrm{D}_{2}^{\circ}\end{array}$ \\
\hline $\begin{array}{r}30 \\
9 \\
20 \\
10 \\
1\end{array}$ & $\begin{array}{l}7900.20 \\
7905.17 \\
7906.10 \\
7908.73 \\
7909.84\end{array}$ & $\begin{array}{l}12654.43 \\
12646.47 \\
12644.98 \\
12640.78 \\
12639.00\end{array}$ & $\begin{array}{ll}b & { }^{3} \mathrm{G}_{4}-z^{3} \mathrm{H}_{5}^{\circ} \\
c & { }^{3} \mathrm{P}_{2}-z^{3} \mathrm{D}_{1}^{\circ} \\
z & \mathrm{P}_{4}^{\circ}-f^{3} \mathrm{~F}_{4} \\
b^{3} \mathrm{G}^{5}-y & { }^{3} \mathrm{~F}_{4}^{\circ} \\
z^{3} & { }^{3} \mathrm{P}_{2}^{\circ}-e^{3} \mathrm{D}_{2}\end{array}$ & $\begin{array}{r}4 \\
1 \\
3 \\
10 \\
1\end{array}$ & $\begin{array}{l}8376.90 \\
8377.62 \\
8384.12 \\
8388.96 \\
8395.68\end{array}$ & $\begin{array}{l}\text { 11934. } 31 \\
11933.29 \\
11924.03 \\
11917.15 \\
11907.62\end{array}$ & $\begin{array}{l}z^{3} \mathrm{G}_{4}^{\circ}-e^{3} \mathbf{F}_{4} \\
z^{7} \mathbf{F}_{3}-e{ }^{3} \mathbf{F}_{4} \\
x^{5} \mathrm{D}_{3}^{\circ}-e{ }^{5} \mathrm{P}_{3} \\
d^{3} \mathbf{F}_{3}-y^{3} \mathbf{F}_{3}^{\circ} \\
b^{3} \mathrm{D}_{2}-x^{3} \mathbf{F}_{3}^{\circ}\end{array}$ \\
\hline $\begin{array}{r}7 \\
60 \\
400 \\
4 \\
3\end{array}$ & $\begin{array}{l}7917.53 \\
7922.97 \\
7924.45 \\
7930.36 \\
7941.08\end{array}$ & $\begin{array}{l}\text { 12626. } 73 \\
12618.06 \\
12615.70 \\
12606.30 \\
12589.28\end{array}$ & $\begin{array}{ll}z & { }^{7} \mathrm{P}_{2}^{\circ}-e^{3} \mathrm{~F}_{2} \\
d & 3 \mathrm{~F}_{2}-y^{3} \mathrm{~F}_{3}^{\circ} \\
z^{3} \mathrm{G}_{5}^{\circ}-f^{3} \mathrm{~F}_{4} \\
z^{3} \mathrm{P}_{1}^{\circ}-\mathrm{F}_{1} \\
c^{3} \mathrm{P}_{1}-z^{5} \mathrm{P}_{2}^{\circ}\end{array}$ & $\begin{array}{r}15 \\
80 \\
4 \\
30\end{array}$ & $\begin{array}{l}8400.60 \\
8435.77 \\
8440.42 \\
8448.69\end{array}$ & $\begin{array}{l}11900.64 \\
11851.03 \\
11844.50 \\
11832.90\end{array}$ & $\begin{array}{ll}a & { }^{1} \mathrm{I}_{6}-z^{3} \mathrm{I}_{6}^{\circ} \\
z^{3} \mathrm{D}^{\circ}-f^{3} \mathrm{~F}_{4} \\
a^{1}{ }^{1} \mathrm{D}_{2}-z \\
z \\
z & { }^{5} \mathrm{P}_{2}-e^{5} \mathrm{~F}_{3} \\
d^{3} & { }^{3} \mathrm{P}_{2}-y^{3} \mathrm{D}_{3}^{\circ}\end{array}$ \\
\hline $\begin{array}{r}10 \\
200 \\
6 \\
200 \\
6\end{array}$ & $\begin{array}{l}7947.97 \\
7948.15 \\
7963.83 \\
7967.88 \\
7994.63\end{array}$ & $\begin{array}{l}12578.37 \\
12578.08 \\
12553.32 \\
12546.94 \\
12504.96\end{array}$ & $\begin{array}{l}z^{5} \mathrm{G}_{3}^{\circ}-e^{3} \mathrm{~F}_{3} \\
z^{3} \mathrm{~F}_{3}^{\circ}-e^{3} \mathrm{~F}_{2} \\
y^{3} \mathrm{D}_{1}^{\circ}-e^{5} \mathrm{P}_{2} \\
z{ }^{3} \mathrm{~F}_{4}^{\circ}-f^{3} \mathrm{~F}_{4} \\
x^{5} \mathrm{D}_{2}^{\circ}-e^{5} \mathrm{P}_{2}\end{array}$ & $\begin{array}{r}2 \\
2 \\
200 \\
9 \\
200\end{array}$ & $\begin{array}{l}8454.92 \\
8456.70 \\
8473.66 \\
8480.60 \\
8483.56\end{array}$ & $\begin{array}{l}11824.18 \\
11821.70 \\
11798.03 \\
11788.38 \\
11784.27\end{array}$ & $\begin{array}{l}a{ }^{3} \mathrm{D}_{3}-z^{5} \mathrm{~F}_{4} \\
x^{5} \mathrm{D}_{2}^{\circ}-e^{5} \mathrm{~S}_{2} \\
z^{3} \mathbf{F}_{2}-e e^{3} \mathbf{F}_{2} \\
z^{7} \mathbf{F}_{5}^{\circ}-e{ }^{5} \mathbf{F}_{5} \\
z^{3} \mathbf{F}_{3}^{\circ}-e^{5} \mathbf{F}_{3}\end{array}$ \\
\hline $\begin{array}{r}2 \\
20 \\
4 \\
1 \\
60\end{array}$ & $\begin{array}{l}7999.28 \\
7999.79 \\
8009.64 \\
8028.28 \\
8036.68\end{array}$ & $\begin{array}{l}12497.69 \\
12496.89 \\
12481.52 \\
12452.54 \\
12439.53\end{array}$ & $\begin{array}{l}a^{3} \mathrm{H}_{4}-z^{7} \mathrm{~F}_{4}^{\circ} \\
c^{3} \mathrm{P}_{2}-y{ }^{5} \mathrm{D}_{3}^{\circ} \\
z^{5} \mathrm{G}_{3}^{\circ}-e^{5} \mathrm{~F}_{4} \\
a^{3} \mathrm{D}_{2}-z^{5} \mathrm{D}_{3}^{\circ} \\
d^{3} \mathrm{P}_{2}-x^{5} \mathrm{D}_{3}^{\circ}\end{array}$ & $\begin{array}{l}6 \\
7 \\
3 \\
2 \\
1\end{array}$ & $\begin{array}{l}8490.45 \\
8494.06 \\
8497.94 \\
8503.42 \\
8506.01\end{array}$ & $\begin{array}{l}11774.70 \\
11769.70 \\
11764.33 \\
11756.75 \\
11753.17\end{array}$ & $\begin{array}{l}a{ }^{3} \mathrm{H}_{5}-z^{5} \mathrm{~F}_{4}^{\circ} \\
z^{5} \mathrm{G}_{4}^{\circ}-e^{3} \mathrm{~F}_{3} \\
a^{1} \mathbf{F}_{3}-y^{3} \mathrm{D}_{2}^{\circ} \\
d^{3} \mathbf{F}_{3}-z^{3} \mathrm{H}_{4}^{\circ} \\
a^{3} \mathrm{D}_{1}-z^{5} \mathrm{D}_{2}^{\circ}\end{array}$ \\
\hline $\begin{array}{l}2 \\
3 \\
2 \\
1 h \\
3\end{array}$ & $\begin{array}{l}8043.36 \\
8049.79 \\
8050.29 \\
8055.05 \\
8058.45\end{array}$ & $\begin{array}{l}12429.20 \\
12419.27 \\
12418.50 \\
12411.16 \\
12405.92\end{array}$ & $\begin{array}{l}c{ }^{3} \mathrm{~F}_{4}-z^{5} \mathrm{P}_{3}^{\circ} \\
d^{3} \mathrm{P}_{2}-z^{3} \mathrm{P}_{1} \\
x^{5} \mathrm{D}_{1}^{\circ}-e^{7} \mathrm{D}_{1} \\
z^{3} \mathrm{P}_{1}-e^{3} \mathrm{D}_{2} \\
a^{3} \mathrm{D}_{1}-z^{5} \mathrm{D}_{1}^{\circ}\end{array}$ & $\begin{array}{l}1 h \\
5 \\
9 \\
1 \\
4\end{array}$ & $\begin{array}{l}8522.21 \\
8523.23 \\
8556.05 \\
8570.84 \\
8585.67\end{array}$ & $\begin{array}{l}11730.82 \\
11729.42 \\
11684.43 \\
11664.26 \\
11644.12\end{array}$ & $\begin{array}{l}z{ }^{7} \mathrm{P}_{3}^{\circ}-e{ }^{3} \mathrm{~F}_{3} \\
y^{3} \mathrm{D}_{3}^{\circ}-e{ }^{3} \mathrm{D}_{3} \\
z^{3} \mathrm{D}_{2}^{\circ}-e{ }^{5} \mathrm{~F}_{2} \\
b^{3} \mathrm{D}_{2}-x{ }^{3} \mathrm{D}_{1}^{\circ} \\
y^{5} \mathrm{P}_{3}^{\circ}-f^{5} \mathrm{D}_{4}\end{array}$ \\
\hline
\end{tabular}


TABle 3-Continued

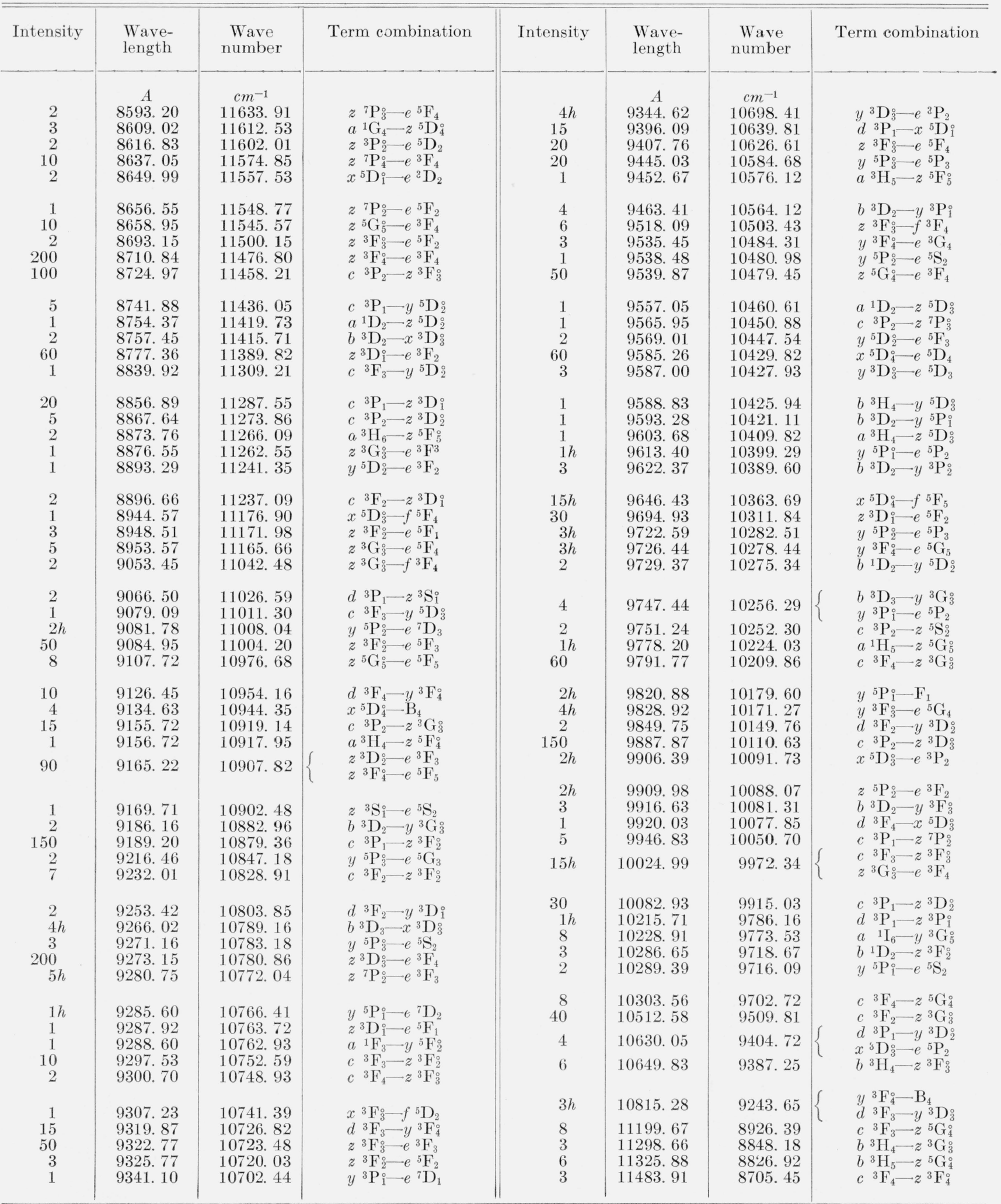


TABLE 4. Strongest unclassified lines

\begin{tabular}{|c|c|c|c|c|c|}
\hline Intensity & Wavelength & Wavenumber & Intensity & Wavelength & Wavenumber \\
\hline $\begin{array}{r}150 \\
80 \\
90 \\
200 \\
100\end{array}$ & $\begin{array}{c}A \\
2279.584 \\
2306.911 \\
2360.530 \\
2420.826 \\
2450.560\end{array}$ & $\begin{array}{c}\mathrm{cm}^{-1} \\
43854.11 \\
43334.68 \\
42350.42 \\
41295.67 \\
40794.65\end{array}$ & $\begin{array}{r}80 \\
200 \\
125 \\
125 \\
100\end{array}$ & $\begin{array}{c}A \\
4806.212 \\
4832.998 \\
4910.236 \\
4919.004 \\
4975.371\end{array}$ & $\begin{array}{c}\mathrm{cm}^{-1} \\
20800.59 \\
20685.31 \\
20359.94 \\
20323.64 \\
20093.40\end{array}$ \\
\hline $\begin{array}{r}150 \\
125 \\
100 \\
100 \\
80\end{array}$ & $\begin{array}{l}2495.691 \\
2560.845 \\
2570.973 \\
2601.456 \\
2676.354\end{array}$ & $\begin{array}{l}40056.98 \\
39037.91 \\
38884.13 \\
38428.53 \\
37353.16\end{array}$ & $\begin{array}{l}300 \mathrm{H} \\
150 \\
200 \mathrm{H} \\
100 \\
600 \mathrm{H}\end{array}$ & $\begin{array}{l}5213.440 \\
5275.087 \\
5334.716 \\
5350.420 \\
5361.792\end{array}$ & $\begin{array}{l}19175.86 \\
18951.76 \\
18739.93 \\
18684.93 \\
18645.30\end{array}$ \\
\hline $\begin{array}{r}250 \\
125 \\
100 \\
75 \\
75\end{array}$ & $\begin{array}{l}2735.669 \\
2753.433 \\
2812.817 \\
2998.346 \\
3059.175\end{array}$ & $\begin{array}{l}36543.32 \\
36307.57 \\
35541.08 \\
33342.00 \\
32679.05\end{array}$ & $\begin{array}{l}500 \mathrm{H} \\
150 \mathrm{H} \\
250 \mathrm{H} \\
500 \\
100\end{array}$ & $\begin{array}{l}5401.047 \\
5439.218 \\
5479.415 \\
5578.412 \\
5603.552\end{array}$ & $\begin{array}{l}18509.78 \\
18379.89 \\
18245.05 \\
17921.27 \\
17840.87\end{array}$ \\
\hline $\begin{array}{r}80 \\
100 \\
125 \\
150 \\
100\end{array}$ & $\begin{array}{l}3226.906 \\
3327.700 \\
3332.051 \\
3693.760 \\
4738.410\end{array}$ & $\begin{array}{l}30980.49 \\
30042.14 \\
30002.92 \\
27064.98 \\
21098.23\end{array}$ & $\begin{array}{r}90 \\
100 \\
300 \\
100 \\
80\end{array}$ & $\begin{array}{l}6528.73 \\
7266.95 \\
7475.40 \\
7529.58 \\
7797.90\end{array}$ & $\begin{array}{l}15312.68 \\
13757.14 \\
13373.53 \\
13277.30 \\
12820.44\end{array}$ \\
\hline
\end{tabular}

Washington, D.C.

(Paper 63A3-17) 